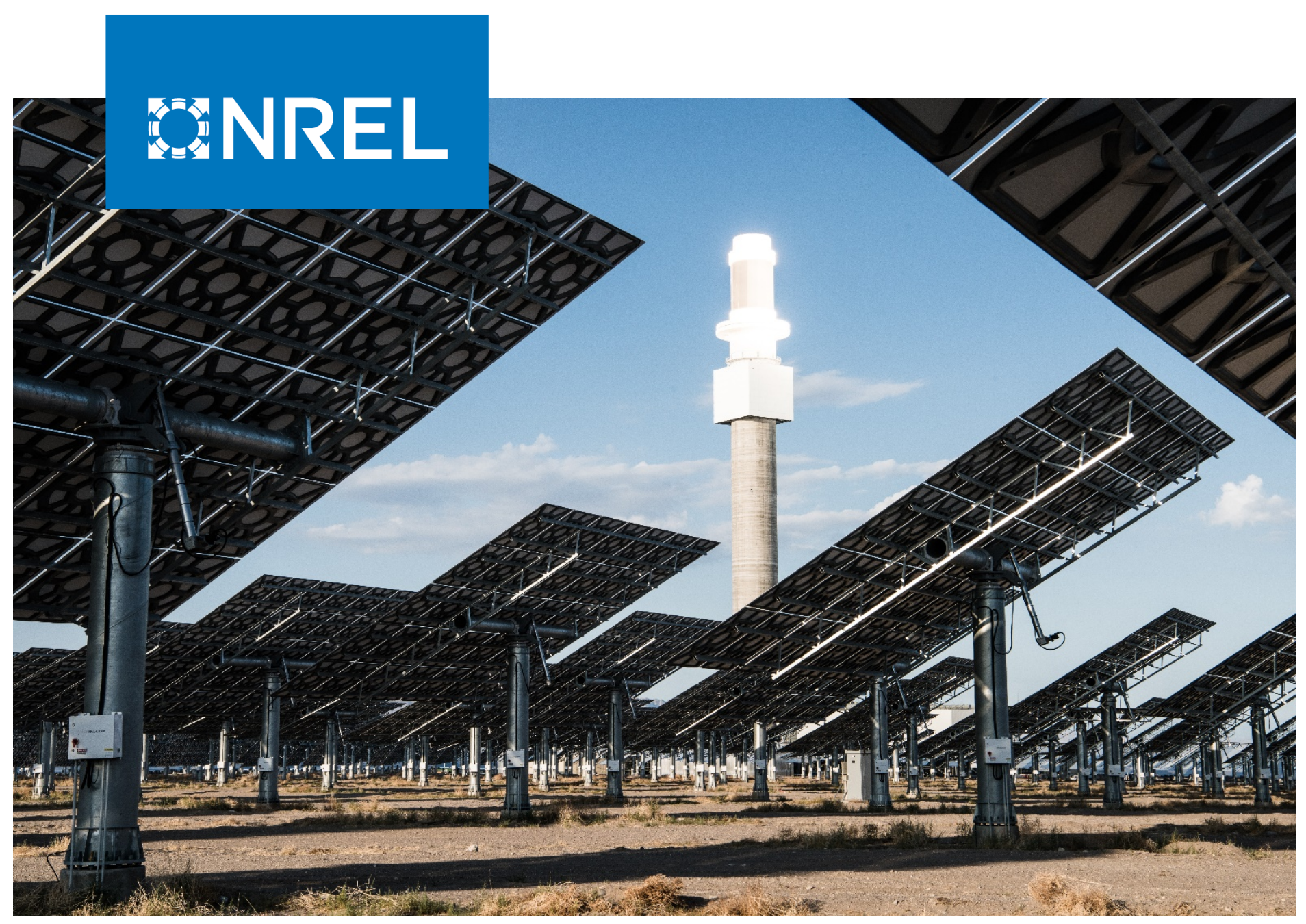

\title{
Concurrent Optimization of Capital Cost and Expected O\&M
}

Michael J. Wagner, ${ }^{1}$ Alexandra Newman, ${ }^{2}$ David Morton, ${ }^{3}$ and Sven Leyffer ${ }^{4}$

1 National Renewable Energy Laboratory

2 Colorado School of Mines

3 Northwestern University

4 Argonne National Laboratory

NREL is a national laboratory of the U.S. Department of Energy Office of Energy Efficiency \& Renewable Energy

Operated by the Alliance for Sustainable Energy, LLC

This report is available at no cost from the National Renewable Energy Laboratory (NREL) at www.nrel.gov/publications.
Technical Report

NREL/TP-5700-79093

February 2021 


\title{
FANEL
}

\section{Concurrent Optimization of Capital Cost and Expected O\&M}

\author{
Michael J. Wagner, ${ }^{1}$ Alexandra Newman, ${ }^{2}$ David Morton, ${ }^{3}$ \\ and Sven Leyffer ${ }^{4}$
}

1 National Renewable Energy Laboratory

2 Colorado School of Mines

3 Northwestern University

4 Argonne National Laboratory

\section{Suggested Citation}

Wagner, Michael J., Alexandra Newman, David Morton, and Sven Leyffer. 2021. Concurrent Optimization of Capital Cost and Expected O\&M. Golden, CO: National Renewable Energy Laboratory. NREL/TP- NREL/TP-5700-79093. https://www.nrel.gov/docs/fy21osti/79093.pdf.

NREL is a national laboratory of the U.S. Department of Energy Office of Energy Efficiency \& Renewable Energy Operated by the Alliance for Sustainable Energy, LLC

This report is available at no cost from the National Renewable Energy Laboratory (NREL) at www.nrel.gov/publications.

Contract No. DE-AC36-08GO28308
Technical Report NREL/TP-5700-79093 February 2021

National Renewable Energy Laboratory 15013 Denver West Parkway Golden, CO 80401 303-275-3000 • www.nrel.gov 


\section{NOTICE}

This work was authored in part by the National Renewable Energy Laboratory, operated by Alliance for Sustainable Energy, LLC, for the U.S. Department of Energy (DOE) under Contract No. DE-AC36-08GO28308. Funding provided by the U.S. Department of Energy Office of Energy Efficiency and Renewable Energy Solar Energy Technologies Office. The views expressed herein do not necessarily represent the views of the DOE or the U.S. Government.

This report is available at no cost from the National Renewable Energy Laboratory (NREL) at www.nrel.gov/publications.

U.S. Department of Energy (DOE) reports produced after 1991 and a growing number of pre-1991 documents are available free via www.OSTI.gov.

Cover Photo by Dennis Schroeder: NREL 46149.

NREL prints on paper that contains recycled content. 


\section{Acknowledgments}

The following team contributed to the composition of this report and the research accomplishments herein:

- Dr. Sven Leyffer, Argonne National Laboratory

- Dr. Jeffery Larson, Argonne National Laboratory

- Professor David Morton, Northwestern University

- Gökçe Kahvecioğlu, Ph.D. Student, Northwestern University

- Professor Alexandra Newman, Colorado School of Mines

- Professor Rob Braun, Colorado School of Mines

- Will Hamilton, Ph.D. Student, Colorado School of Mines

- Jesse Wales, Ph.D. Student, Colorado School of Mines

- Jennifer DiCarlo, Ph.D. Student, Colorado School of Mines

- Dr. Michael Wagner, National Renewable Energy Laboratory

- Dr. Alex Zolan, National Renewable Energy Laboratory

- Dr. Janna Martinek, National Renewable Energy Laboratory

- Dr. Craig Turchi, National Renewable Energy Laboratory

The authors gratefully acknowledge the support and collaboration of Jolyon Dent and Charles Diep whom offered valuable industry guidance during their time at SolarReserve. 


\section{Table of Contents}

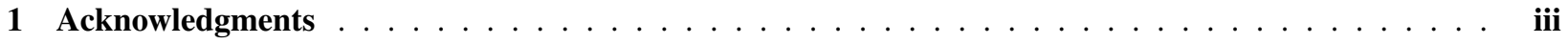

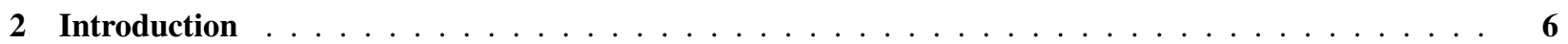

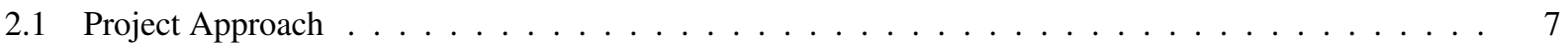

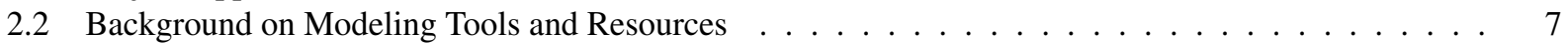

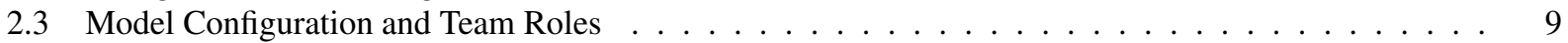

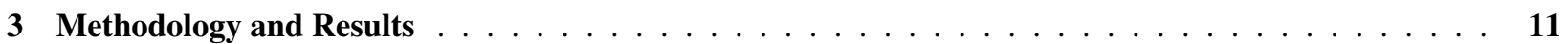

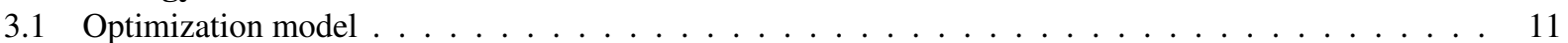

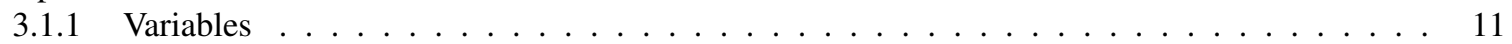

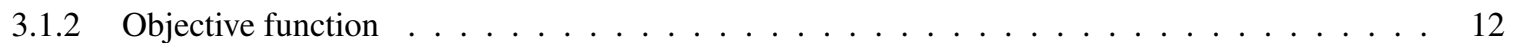

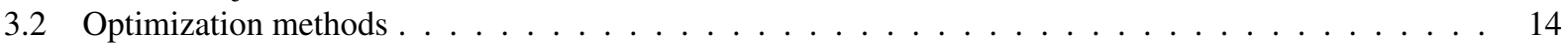

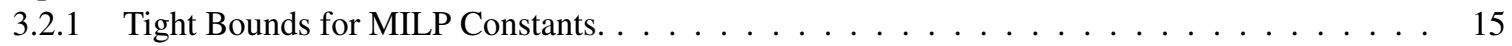

3.2.2 Heuristics for Accelerating the Creation of Cutting Planes . . . . . . . . . . . . . . . . 18

3.2.3 New Trust-Region Method for Integer Optimization _ . . . . . . . . . . . . . . . . . . . . 18

3.2 .4 Performance Improvements . . . . . . . . . . . . . . . . . . . . . . . . . . . 19

3.2 .5 Solver Implementation Summary $\ldots \ldots \ldots \ldots$

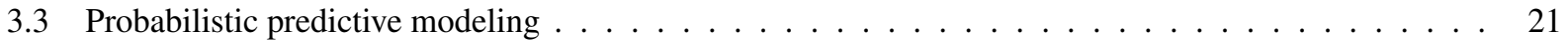

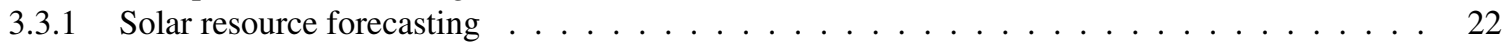

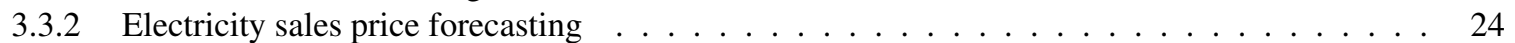

3.3 .3 Heliostat field availability $\ldots \ldots \ldots \ldots \ldots \ldots$

3.3 .4 Soiling and degradation $\ldots \ldots \ldots \ldots \ldots \ldots$

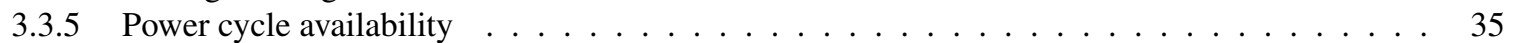

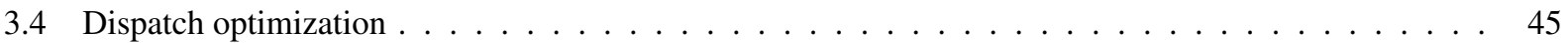

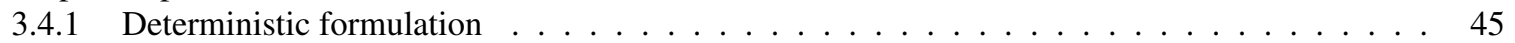

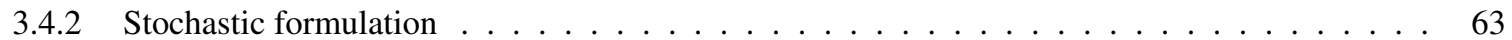

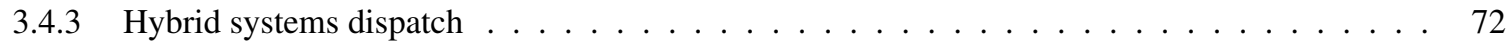

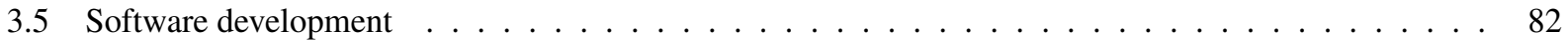

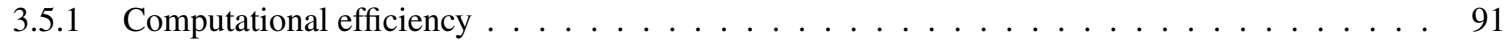

3.5 .2 Open-source code . . . . . . . . . . . . . . . . . . . . . . . . . 97

3.5.3 Application interface . . . . . . . . . . . . . . . . . . . . . 97

3.5.4 Overall system design and operation . . . . . . . . . . . . . . . . . . . . . . . . 105

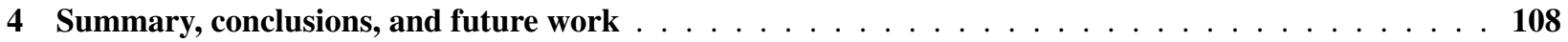

\section{List of Figures}

Figure 1. A molten-salt power tower system (Image credit: NREL). $\ldots \ldots \ldots \ldots \ldots$ 
Figure 2. Summary of the optimization model configuration and team roles within the project. Topical areas and models are shaded according to the organization that is primarily contributing. Models are deterministic except where noted as stochastic. . . . . . . . . . . . . . . . . . . . 9

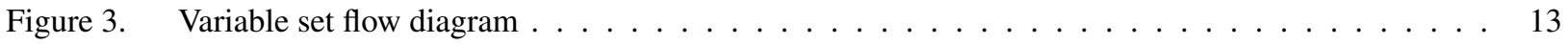

Figure 4. Graphs showing the number of feasible combinations of $n+1$ points and the secants that pruned at least one 'potential' point at an iteration for $n=3$ and $n=4 \ldots \ldots \ldots \ldots \ldots \ldots$

Figure 5. Graphs showing the number of points that formed 'useful' secants, those which pruned at least one 'potential' point at an iteration for $n=3$ and $n=4 \ldots \ldots \ldots \ldots \ldots \ldots$

Figure 6. Convergence of optimality gap (left) and wall-clock time (right) for trust-region (dashed) versus traditional approach $($ solid $) \ldots \ldots \ldots \ldots \ldots \ldots \ldots$

Figure 7. Improvement in wall-clock solution time with different cutting plane models. Blue and red bars show the MIP model using CPLEX, the yellow bars are the simple enumeration scheme, and the green and brown bars correspond to the enumeration scheme with heuristics and with trust region added. . . . .

Figure 8. Boxplots of hourly LMP DAM price data over 2015-2017 in months January, April, July, and October for node CONTADNA_1_N001. . . . . . . . . . . . . . . . . . . .

Figure 9. Hourly solar field availability for a case study using 10 O\&M staff; each line denotes a different repair prioritization policy. Component lifetime and repair time distributions are described in Table 7 . . .

Figure 10. Sensitivity plot showing the optimal number of wash crews as a function of changing parameter values; base values are available in Table $9 \ldots \ldots \ldots \ldots \ldots$

Figure 11. Solution development by the optimization model $(\mathcal{R})$ and simulation model $(\mathcal{S})$, which obtain optimal dispatch and simulated failure and maintenance events over a rolling horizon. . . . . . . . .

Figure 12. The pricing profile used with the repeated experiment, showing the first 48 hours of the one-year

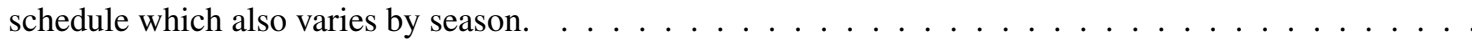

Figure 13. Results of sales and failures by cycling cost, shown in parts (a) and (b), respectively. As the cycling cost is increased, there is a point when sales are maximized and the number of failures stop declin-

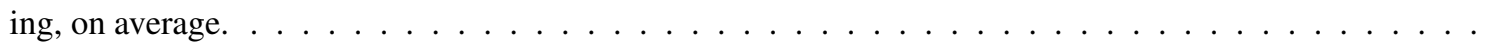

Figure 14. The number of failures are directly related to the number of startups. The four groups of startups are a result of the four cycling costs, with higher cycling costs associated with fewer startups. . . . . . . .

Figure 15. Cycle efficiency as a function of input thermal power represented using a piece-wise linear func-

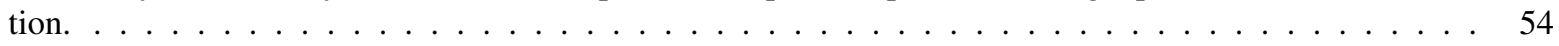

Figure 16. The impact of time horizon length (hours) on annual energy production and PPA price. . . . . . . 55

Figure 17. Information flow in the SAM-MSPT model. The MIP formulation is solved as a simultaneous set of equalities and inequalities, and the hourly solution profile is used by the CSP Controller to set target power production levels and operational states over the subsequent operational time horizon. . . . . . . .

Figure 18. Market pricing scenarios presented by Guédez et al. These tariff schedules are implemented to determine the impact of dispatch optimization on system sizing. . . . . . . . . . . . . . . . .

Figure 19. Comparison of performance profiles for the pool price tariff schedule. Plots (a) and (b) show traces of the TES charge state for each day of the year. Plots (c) and (d) show box-whisker plots of daily electricity production variability over a year grouped by hour of the day. Each box indicates the mean annual electricity generation by hour, the first and third quartile limits (box limits), and two times the interquartile range (whiskers). "Outliers" are shown as blue dots. Summer (red) and winter (blue) tariff multipliers are overlaid on each plot. . . . . . . . . . . . . . . . . . 
Figure 20. Plant power generation profile with varying change in production penalty values, $C^{\delta \dot{W}}$. (Penalties are given in the legend.) $\ldots \ldots \ldots \ldots \ldots \ldots \ldots \ldots$

Figure 21. Impact of production change cost penalty on number of turbine cycles per day, annual energy generation, and PPA price for two pricing scenarios - a generic summer afternoon peak schedule (Default) and a morning/evening double-peak (Peaker) schedule. Annual energy and PPA price are shown as fractional values relative to the lowest-penalty case . . . . . . . . . . . . . . . . .

Figure 22. Number of cycle starts per year, annual energy output, and PPA price for the Default case with

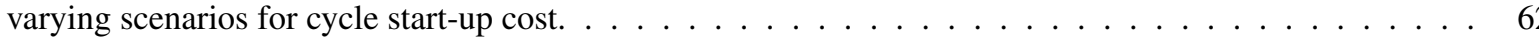

Figure 23. Dispatch profiles over Feb 11-15 on the synthetic dataset . . . . . . . . . . . . . . . . 69

Figure 24. Illustration of 48 -hr rolling horizon scheme with $6-\mathrm{hr}$ time increments. . . . . . . . . . . 71

Figure 25. CSP-PV hybrid system design and simulation flow chart. CSP and PV simulations use SAM for

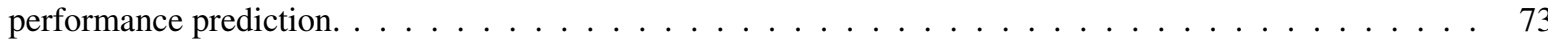

Figure 26. System layout consisting of CSP (with TES), PV field generation, and battery storage . . . . . . . 74

Figure 27. Example gross electric generation profiles using dispatch optimization (a) with objective function (3.50) and (b) with objective function (3.51). The left axis represents the dependent variables associated with gross electric power generation (positive) and storage (negative) from the individual plant sub-systems over the time horizon. The sub-systems include: CSP power cycle (gray), PV field (orange), battery discharge (green), PV field curtailment (red), battery charging by the power cycle or grid (black), and battery charging by the PV system (blue). PV curtailment (in red) represents lost energy. The right axis represents the following dependent variables: normalized thermal energy storage (red line), battery state of charge (SOC) (blue line), and PPA price multiplier (black line).

Figure 28. The original formulation has sub-hourly time intervals throughout the time horizon. Version A possesses sub-hourly time intervals during the first day and aggregates data to hourly fidelity for the second day. Version B possesses sub-hourly time intervals for a time window less than a full day, and requires multiple solves to produce a day-one solution - in this example, four six-hour solves using five-

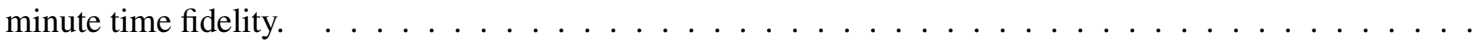

Figure 29. The distribution of dispatch problem solve times for a year's worth of time periods for the original (left) and Version A (right) formulations . . . . . . . . . . . . . . . . .

Figure 30. Sort histograms to compare direct normal irradiance and market structures of the full-factorial

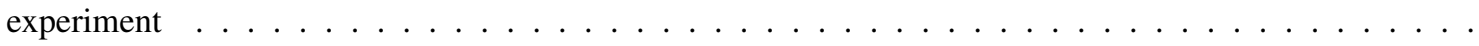

Figure 31. Time-of-use variation of electricity prices for the Northern Chile spot (NC spot) and Pacific Gas \& Electric $(\mathrm{PG} \& \mathrm{E})$ market. . . . . . . . . . . . . . . . . . . . . . . . . . .

Figure 32. Annual solution attributes for the full-factorial experiment; annual sales, solve time, annual generation, cycle starts, cycle ramping, and battery lifecycles. . . . . . . . . . . . . . . .

Figure 33. Clustered 2-day DNI profiles (including previous day). Bold lines indicate the cluster exemplar

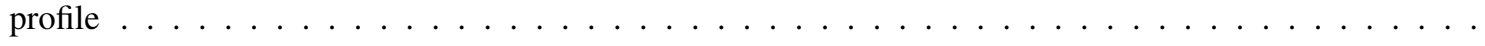

Figure 34. Comparison of full and clustered simulations at 25 randomly selected independent variable combinations (displayed in order of decreasing cash flow) . . . . . . . . . . . . . .

Figure 35. Cash flow error from clustered simulations at 25 randomly selected independent variable combinations. Dashed black lines represent $1 \%$ of the highest cash flow from the set of full simulations. . . . . 94

Figure 36. Simulation time relative to full annual simulation. . . . . . . . . . . . . . . . . . 95

Figure 37. Yearly performance for Daggett, CA with a block electricity price schedule . . . . . . . . . 95

Figure 38. Yearly performance for Rice, CA with CAISO locational marginal prices from 2015 . . . . . . 96 
Figure 39. Screenshot of the application's main UI window; labels describe the different components of the

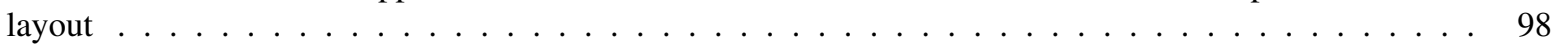

Figure 40. Screenshot of the application's variable dialog, which is accessible by selecting the button labeled Variables in the scripting window . . . . . . . . . . . . . . . . . . .

Figure 41. Screenshot of the application's scripting window, with commands used to set and get the variables tower_exp and h_tower; these commands are automatically generated by selecting the Insert SET and Insert GET links in the variable dialog to set and return each variable's value, respectively . . . . 100

Figure 42. Screenshot of the program's function reference, which is accessed by selecting Help in the main

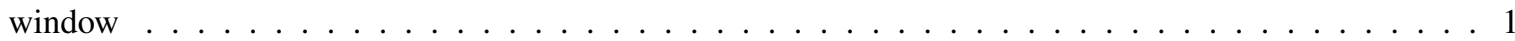

Figure 43. Screenshot of the Data tab, in which the search bar is used to select and view a time-series of net power generation in the right-hand side of the window; previously selected outputs that do not fit the search criterion (i.e., price signal) remain in view . . . . . . . . . . . . . . . . . . 102

Figure 44. Screenshot of the Timeseries viewer window displaying a table of summary statistics for net

Figure 45. Screenshot of the Timeseries viewer window displaying a time-series plot of the price signal and net power generation . . . . . . . . . . . . . . . . . . . .

Figure 46. Screenshot of the Timeseries viewer window displaying a monthly profile of net power generation 104

Figure 47. Screenshot of the Timeseries viewer window displaying a heat map of net power generation . . . 104

Figure 48. Results of system optimization over a range of nominal power cycle conversion efficiencies. The plot shows the starting-point value in black and the best value in green. The final value at convergence is shown in dotted blue (often covered by the green lines) . . . . . . . . . . . . . . . . . . . .

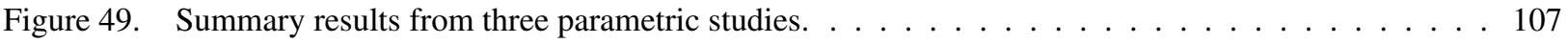

\section{List of Tables}

Table 1. Variable Names and Description . . . . . . . . . . . . . . . . . . . . . 11

Table 2. Functional dependence for each decision variable. Shaded cells indicate whether a particular function should be re-evaluated upon the change in a particular variable value. . . . . . . . . . . . . . 12

Table 3. Candidate exogenous variables. . . . . . . . . . . . . . . . . . . . . . 24

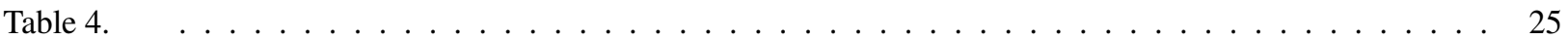

Table 5. Input parameters used by the heliostat availability model. Parameter and variable names match those used in DAO-tk scripting interface . . . . . . . . . . . . . . . . . . . . . . 28

Table 6. Output parameters generated by the heliostat availability model. Parameter names match those used in DAO-tk scripting interface . . . . . . . . . . . . . . . . . . . . . . . . . .

Table 7. Component parameters for model demonstration . . . . . . . . . . . . . . . . . . . . . 29

Table 8. Components of the wash crew subproblem formulation . . . . . . . . . . . . . . 31

Table 9. Inputs to case studies for soiling and degradation models . . . . . . . . . . . . . . . . . . . 34

Table 13. Parameters and sets used in $(\mathcal{R}) \ldots \ldots \ldots$. . . . . . . . . . . . . . . . . . . 49

Table 17. Parameters and sets. . . . . . . . . . . . . . . . . . . . . . 63 
Table 10. Mean field reflectivity (Refl.), mean number of mirror replacements (NR), and point and interval estimates for the mean relative loss reduction (LR) as a function of replacement and washing policies $\quad \ldots \quad 84$

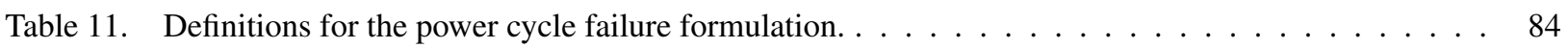

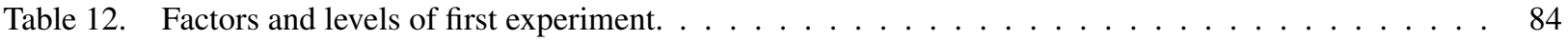

Table 14. Variables used in $(\mathcal{R}) \ldots \ldots \ldots \ldots \ldots \ldots \ldots$

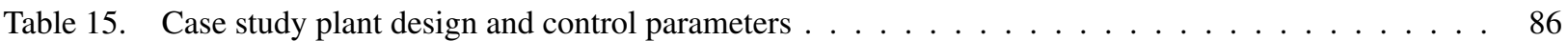

Table 16. Characteristics for each market scenario in which PPA price is at a minimum value, both for heuristic $(\mathrm{H})$ and optimized $(\mathrm{O})$ dispatch $\ldots \ldots \ldots \ldots \ldots \ldots$

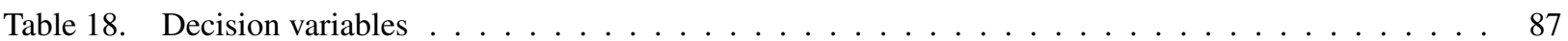

Table 19. Revenue for one year derived from the expected value problem (EV), the stochastic program (SP), and the policy approach using synthetic data. The percentage values are the increase over EV . . . . . . 87

Table 20. Revenue derived from the expected value problem (EV), the stochastic program (SP), and the policy approach using historical data from Hanford, California. The percentage values are the increase over

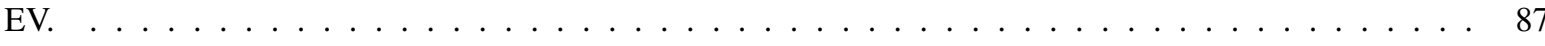

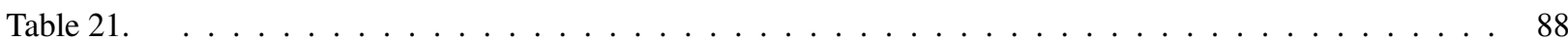

Table 22. Relative gap (\%) in PPA prices over perfect information on RTM15 price forecasts for SOLRAD data for Hanford, California. Both the price point forecast and the historical mean use model (3.49) with $\lambda=0$, and both approaches benefit from the conditional DNI point forecast. The values mean that relative to a perfect information PPA price, the price grows by $9.60 \%$ under the conditional point forecast

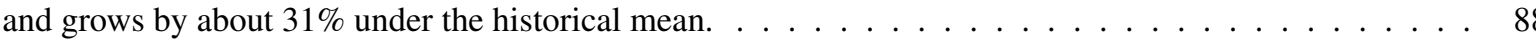

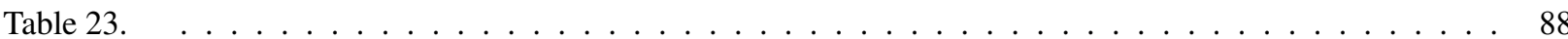

Table 24. Comparison of annual hybrid simulation metrics between the dispatch model with objective function (3.50) and (3.51). ${ }^{\dagger}$ Solve times are based on Quad-Quad core CPUs at 2.93 GHz 32GB RAM 1TB HDD hardware. . . . . . . . . . . . . . . . . . . . . . . . .

Table 25. CSP-PV hybrid sub-system operating cost ranges based on literature (references provided in brackets) or engineering approximations and the current value of each cost, given in units of $\$ / M W h . \quad \ldots \quad \ldots 88$

Table 26. Case study plant design . . . . . . . . . . . . . . . . . . . . . . . . . . . 89

Table 27. Solar global horizontal, direct normal, and diffuse horizontal irradiance year totals for N. Chile and S. Nevada. . . . . . . . . . . . . . . . . . . . . . . . . 89

Table 28. Comparison of advantages and disadvantages for different user interface options _ . . . . . . . 90

Table 29. Key parameters used for the case studies . . . . . . . . . . . . . . . . . . . 105

Table 30. Optimization variables solved for each case and parameters that are varied for each case study. . . 105 


\section{Introduction}

Concentrating solar power (CSP) technologies can utilize heat from concentrated sunlight from a field of tracking mirrors to generate electricity, reform fuel, provide process heat, or augment fossil plant heat sources. The most common CSP conversion systems generate electricity using conventional steam turbines in a Rankine cycle, though power cycles using supercritical carbon dioxide $\left(\mathrm{s}-\mathrm{CO}_{2}\right)$ may represent the most promising path forward (Dunham and Iverson, Turchi, Ma, and Dyreby). Among the four major CSP technologies - Parabolic Trough, Linear Fresnel, Dish Stirling, and Power Tower - the lattermost (also called a "Central Receiver" system and illustrated in Figure 1 ) has greatest potential for efficiency improvement and cost reduction (Margolis, Coggeshall, and Zuboy). The first US commercial power tower facilities are only recently coming online with Bright-Source ${ }^{\mathrm{TM}}$ 's Ivanpah I-III facilities and SolarReserve ${ }^{\mathrm{TM}}$ 's Crescent Dunes facility. These facilities represent an important step for CSP in the US, but the relative scarcity of power tower facilities worldwide leaves a dearth of knowledge on operations and maintenance $(\mathrm{O} \& \mathrm{M})$ costs, performance impacts, and operating strategies to minimize cost of energy.

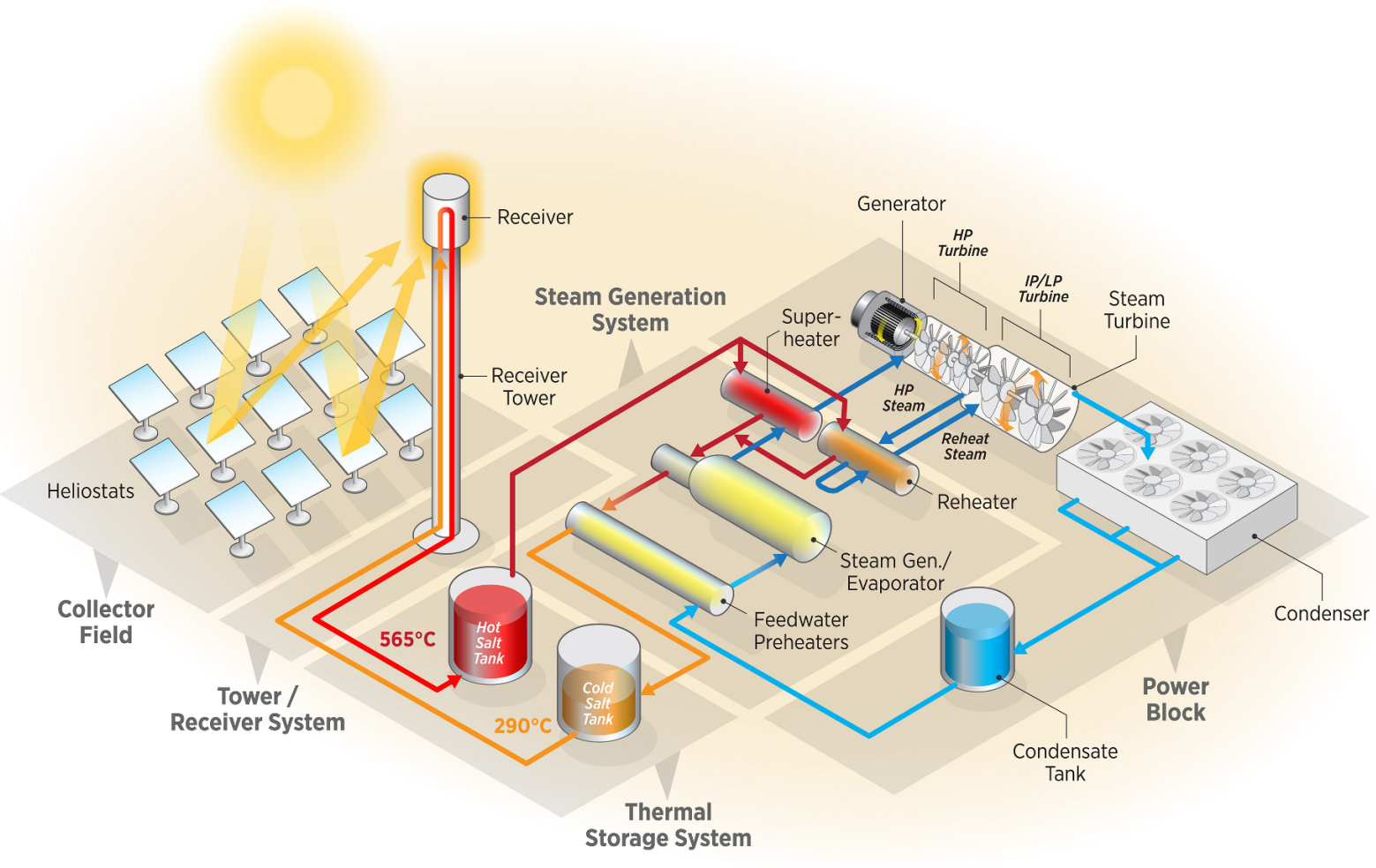

Figure 1. A molten-salt power tower system (Image credit: NREL).

Electricity-generating power tower systems focus light from thousands of independent heliostats onto a thermal receiver, which uses the focused light to warm a heat transfer fluid (HTF), typically, a molten nitrate salt. The HTF is then sent to a power generation cycle or diverted into thermal energy storage (TES) for later use. Although power tower systems can achieve capacity factors approaching base-load status (for example, the Gemasolar facility in Fuentes de Andalucía, Spain (Lata, Rodríguez, and Lara)), US market structures often preferentially value energy production during peak demand hours of the day. Hence, cost-effective solutions typically operate diurnally with potential for multiple, daily production cycles. Because the lifetime of power generation equipment is highly sensitive to heating and cooling rates, cycle startup and grid synchronization typically requires between 1-3 hours, depending on the extent to which equipment has cooled during down-time. 
Thermal storage is - in principle - a straightforward proposition. However, optimal utilization of a TES resource is complex and multi-faceted: thermal energy may be dispatched to produce electricity immediately upon first availability, or thermal energy may be reserved for next-day peak periods at risk of filling storage and dumping energy, or a portion of the thermal energy can be reserved to maintain equipment temperatures, reducing power cycle startup time, etc. Many possible dispatch permutations variously emphasize producing peak power, operating through transients, expediting daily startup, etc. The best operation strategy can change day-to-day throughout the year, depending on the weather and market pricing forecasts.

Several important design and operational decisions are not typically at the forefront of technology development but lie at the heart of what motivates CSP design. They arise due to the close interrelation among subsystems, and due to environmental operating conditions, especially when considering the potentially revolutionary subsystem technologies targeted by the US Department of Energy's SunShot initiative. Specifically, considerations in optimal system design include: (i) the number of heliostats to be purchased considering degradation profiles, soiling rates, mirror washing procedures, parasitic power requirements, and labor rates; (ii) thermal storage dispatch strategy (Denholm and Mehos), accounting for cycle startup time and cumulative damage to the power cycle equipment, (iii) the sizing thermal storage, the power cycle, and the solar field, and (iv) receiver and tower geometry. Finally, CSP - though having a significant operational advantage due to TES - must also develop tools that respond to energy production uncertainty in the near (24-72 hour) time-horizon.

Previous work has shown that careful energy system design and dispatch can reduce costs, e.g., for an appropriately retrofitted building relative to reliance solely on the grid (Pruitt, Braun, and Newman). Rather than performing an expensive retrofit, a more cost-effective and, indeed, imperative, approach given current market competition for lowcost energy systems calls for an immediate, structured optimization effort regarding CSP. Other researchers have shown that significant $O \& M$ cost reduction is possible from a rigorous accounting of O\&M costs (Cohen, Kearney, and Kolb).

\subsection{Project Approach}

Several key outcomes are targeted by the project. First, we develop a set of modeling tools that capture the performance, maintenance, operations, cost, and forecasting aspects of CSP molten salt power tower technology. These models leverage existing tools such as System Advisor Model (SAM), SolarPILOT ${ }^{\mathrm{TM}}$, and those developed in literature, but also involve significant new development, particularly with respect to O\&M considerations such as dispatch operations, direct normal irradiance (DNI) and price forecasting, and component degradation.

Second, we examine and develop techniques for algorithmic optimization of both plant dispatch and of system design. We pose the CSP design and operations analysis in the form of an objective function whose variables are subject to several constraints, and we then seek to minimize the power purchase agreement (PPA) price associated with the objective function. The optimization problem at hand involves computationally expensive evaluations at each point, and information about the rate of change of the objective function with respect to each variable (function derivatives) are not explicitly available and must be calculated. This, in combination with stochastic behavior, implicates a complex and difficult optimization problem. Therefore, we undertake additional development of optimization techniques that enable solving this class of problem.

Finally, we will use the resulting tools to elucidate the ordinarily overlooked trilateral relationship between designpoint criteria, initial component cost, and lifetime O\&M costs. A rigorous modeling and solution approach emphasizes a clear and repeatable methodology, providing insight into the nature of the relationships while leaving the detailed engineering and costing exercises to the technology developers who are best suited for that work. Ultimately, this project will deliver a tool and methodology for use by entities that have access to proprietary design and cost information.

\subsection{Background on Modeling Tools and Resources}

Exploring the tradeoff between technology cost and performance is possible with robust technology simulation software in which the detail of each subsystem is captured within the context of the entire power plant. The National 
Renewable Energy Laboratory (NREL) develops software tools for characterizing subsystem cost and performance (e.g., SolarPILOT) and for predicting the productivity of the integrated power plant over the course of a year using measured weather data (e.g., SAM Gilman and Dobos). These essential tools provide a framework for accurately and quickly quantifying the impact of design and operational decisions.

SolarPILOT and SAM span the range of analysis from initial design to simulation and financial evaluation. SolarPILOT is a unique tool for layout, optimization, and optical characterization of the heliostat field. The software is capable of evaluating the tradeoff between heliostat cost and optical performance, the impact of mirror soiling and washing schedules both globally and in local regions within the heliostat field, and of selecting solar field design variables that minimize the expected cost of energy. SolarPILOT is available as a stand-alone software package and is utilized via an application programming interface as the optical simulation engine in SAM. The SAM software combines performance, cost, and financial models for a variety of renewable energy technologies, and is used to predict total plant and subsystem productivity, detailed component behavior, and financial metrics including cost of energy, power purchase agreement price, and internal rate of return.

SAM's CSP technology performance models are derived from a combination of engineering physical principles and (semi-)empirical correlations. The SAM Molten Salt Power Tower (SAM-MSPT) model (Wagner) uses a streamlined version of the SolarPILOT optical simulation engine and detailed thermal models of the receiver, thermal storage, and power cycle subsystems. SAM has received continuous funding from the DOE since 2006, and currently SAM-MSPT supports a generalized and stable plant control algorithm and implements a short-time-horizon (deterministic) TES dispatch optimization model.

The outcome of a SAM-MSPT simulation typically consists of several performance and financial metrics of interest - typically PPA price - though many different flavors of this metric exist. The process for obtaining such a metric involves a series of carefully orchestrated procedures. The first step is to define the input parameters, including system sizing, optical and thermodynamic performance characteristics, component geometries, and costs. Next, the SAM-MSPT model evaluates the system to determine an improved design relative to that of the baseline system, manipulating receiver height and diameter, tower height, and heliostat field layout positions. This step utilizes the SolarPILOT optical simulation engine to balance the optical performance of the heliostat field against costs using a simplified capital cost and annual productivity estimate.

In the third step, SAM-MSPT invokes the SolarPILOT engine to characterize the optical efficiency and concentrated solar flux intensity on the receiver surface (which is a primary factor in thermal performance and receiver lifetime) at a selected set of sun positions throughout the year. The resulting data sets serve as surrogate models that can be quickly evaluated at each time step in a subsequent annual simulation, greatly improving simulation speed and adding little error to energy cost estimates. Next, SAM-MSPT evaluates the system over each hour of a "typical meteorological year" using aggregated weather data from the selected location. This model is perhaps the most complex of any in the set, as behavior in each subsystem is closely tied to the control algorithm, behavior in connected subsystems, and the energy state of the system in the previous time step. For example, the thermal efficiency of the power cycle depends on the temperature of the hot HTF entering the heat exchanger, and this temperature can vary with the source of the fluid (receiver or TES system, or a mixture thereof), or thermal losses in TES or other connection piping. In turn, the thermal efficiency of the receiver depends on how efficiently heat is utilized in the power cycle. Many implicit interconnections play an important role in determining the overall performance of the plant. Once the annual hourly simulation is complete, SAM-MSPT then calculates revenue, discounted lifetime costs, and financial metrics of interest.

Appropriate modeling of system uncertainties is key when optimizing the design of engineering-based systems. This involves capturing in a probabilistic model, for example, randomness associated with pricing or weather forecasts. If such a probabilistic model is posited, fit with relevant data coupled with expert knowledge, and the model's goodness-of-fit is deemed reasonable, then this model can be incorporated within a stochastic optimization model such as a stochastic program. This project characterizes the impact of stochastic weather and market price processes on plant performance and design. 


\subsection{Model Configuration and Team Roles}

Figure 2 presents the relationship between the model used in this project and the role of each project group. The four research institutions participating in the project include Argonne National Laboratory (Laboratory for Advanced Numerical Simulations), Colorado School of Mines (Department of Mechanical Engineering), Northwestern University (Center for Optimization and Statistical Learning), and the National Renewable Energy Laboratory (Thermal Sciences) who are leading the project. SolarReserve is an industrial partner in an advisory role, assisting in the collection of data and model verification.

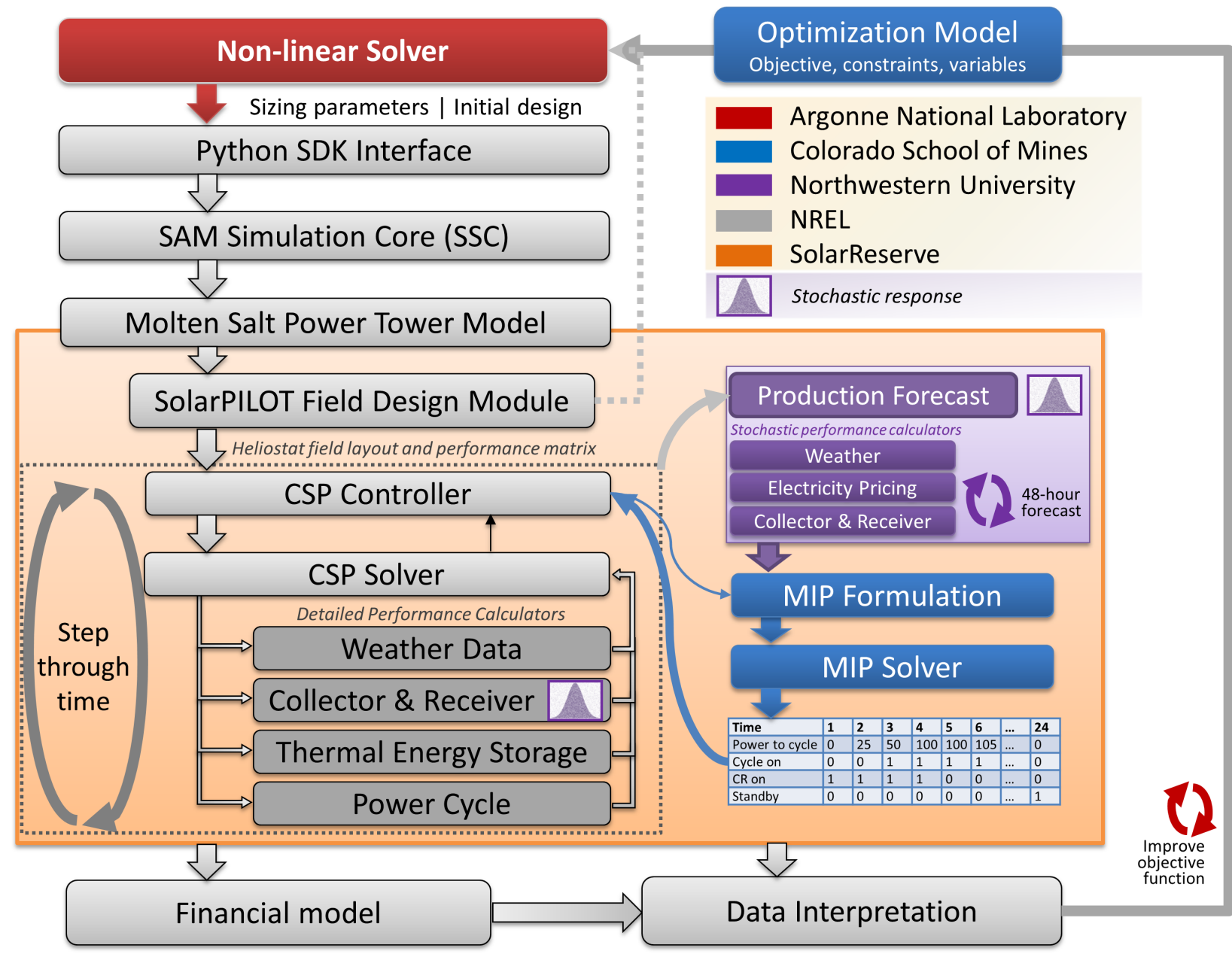

Figure 2. Summary of the optimization model configuration and team roles within the project. Topical areas and models are shaded according to the organization that is primarily contributing. Models are deterministic except where noted as stochastic.

The diagram presents the relationships and layers that exist between separate efforts in this project. At the highest level, an outer optimization loop seeks to determine the optimal values for the set of design and operation parameters previously discussed. The outer loop consists of an objective function and constraining relationships, an algorithm and methodology for systematically improving that objective function, and a set of "black-box" simulation and design modules (SAM, SolarPILOT) that evaluate the performance of a posed design instance. Objective function variables such as number of O\&M staff, mirror washing frequency, subsystem sizing, etc., are related to the objective function value both explicitly and via the black-box tools that evaluate performance, as described elsewhere in this report. 
The outer-loop constraints define relationships between the optimization variables and other performance model inputs, among the optimization variables themselves, and between design variables and engineering limits that must be imposed. For example, an optimization variable "number of O\&M staff" may impact the cost of O\&M, the lifetime of plant components, and the fraction of the heliostat field that is available at a given time. Several constraints must be put in place to define these relationships so that a change to the optimization variable results in an appropriate change to cost, lifetime, or availability inputs.

Figure 2 also shows an "inner-loop" optimization problem that determines the optimal day-to-day energy dispatch profile given a particular system design, pricing schedule, and weather forecast. The inner-loop optimization is formulated as a mixed-integer linear program (MIP), and is an approximation of the detailed SAM-MSPT performance calculators that run as part of SAM annual simulations. The program runs once for each day of the annual simulation, providing an operation schedule to the SAM control algorithm that maximizes revenue subject to a number of constraints.

Lastly, Figure 2 shows the aspects of the modeled system that we treat as stochastic. Weather, pricing, and solar field performance predictions are subject to uncertainty because of the chaotic nature of future events. The performance of the solar field is predictable to the extent that weather conditions are known, but field equipment is also subject to periodic failure, and the future availablity of heliostats cannot be known exactly. Consequently, this work attempts to treat these aspects of uncertainty as part of the optimization process so that the system design can account for the realities of plant operation over time. 


\section{Methodology and Results}

\subsection{Optimization model}

This project defines an optimization model that relates design and operations variables to an objective function and constraints with a goal of improving overall techno-economic viability of the technology at hand by simultaneously manipulating variable values while evaluating the resulting impact on the objective - PPA price. Note that this project addresses several optimization problems, including the aforementioned design problem, a separate but related dispatch optimization problem, and several others. For the sake of clarity, the broadest design problem is referred to as the "outer loop." The process of evaluating the outer loop is illustrated in Figure 2 above.

\subsubsection{Variables}

We select a set of decision variables that affect the performance and cost of the system either with respect to initial capital investment or longer-term operational and maintenance requirements. The variables reflect real decisions that must be made about subsystem sizing, geometrical configuration, policy for replacement or restoring equipment, or other matters. Variables relate to one another by their effect on the objective function and by constraints; for example, a change in the tower height variable value may warrant changes to the design-point DNI value, receiver geometry, or solar multiple in order to achieve an improvement in the objective function value. Hence, we have selected a variable set (Table 1) that enables simultaneous optimization of various aspects of plant design and performance, exploring trade-offs between initial design, O\&M, and productivity. Variables may have either continuous or integer domains, and this characteristic affects the methodology by which the variable values are optimized.

Table 1. Variable Names and Description

\begin{tabular}{|c|c|c|l|}
\hline Symbol & Units & Type & Description \\
\hline h_tower & $\mathrm{m}$ & Continuous & Tower height \\
\hline D_rec & $\mathrm{m}$ & Continuous & Receiver diameter \\
\hline rec_height & $\mathrm{m}$ & Continuous & Receiver height \\
dni_des & $\mathrm{kW} / \mathrm{m}^{2}$ & Continuous & Receiver DNI design point \\
solarm & - & Continuous & Solar multiple \\
\hline design_eff & - & Continuous & Cycle design point conversion efficiency \\
P_ref & $\mathrm{kW}$ & Continuous & Cycle design point power output \\
tshours & hours & Continuous & Hours of full-load power generation from TES (TES capacity) \\
\hline degr_replace_limit & - & Continuous & Mirror degradation replacement threshold \\
\hline N_panels & - & Integer & Number of panels per heliostat \\
om_staff* & - & Integer & Number of full-time staff available for heliostat repairs \\
\hline n_wash_crews & - & Integer & Number of full-time staff available for mirror washing \\
\hline Value optimized in dedicated sub-problem & \\
\hline
\end{tabular}

Each variable affects the objective function (presented in Section 3.1.2 below) differently with respect to the optimization objective and the set of sub-models that incorporate on the variable. The objective function, which we call " $\mathcal{Z}$ ", is composed of seven separate but interrelated sub-models, including the:

i) explicit terms model $(\mathcal{E})$,

ii) solar field design and characterization model $(\mathcal{D})$,

iii) heliostat mechanical failure and availability $\operatorname{model}(\mathcal{M})$,

iv) heliostat optical degradation and soiling model $(\mathcal{O})$,

v) annual performance simulation model $(\mathcal{S})$,

vi) cycle component availability model $(\mathcal{C})$, and the

vii) financial power purchase agreement $(\mathrm{PPA})$ price model $(\mathcal{F})$. 
Variables are classified according to model dependency, as shown in Table 2, facilitating sub-problem optimization in which an algorithm can quickly assess the impact of certain variables on the objective function without requiring a full set of model evaluations. Note that inclusion in a set indicates that the full objective $\mathcal{Z}$ cannot be determined without first evaluating the affected sub-model(s). For example, a change in the number of hours of thermal storage $\left(x^{\text {hours,tes }}\right)$ requires execution of only the performance simulation $\mathcal{S}$, while a change in tower height $\left(x^{h, t o w e r}\right)$ implicates every sub-model and is thus much more computationally expensive to optimize. Note that variables denoted "*" receive special treatment, as dedicated sub-problem models are solved to determine the optimal value at each iterate. Therefore, although these items are considered variables for the purposes of describing the optimization problem, in practice, they are not ultimately independent.

Table 2. Functional dependence for each decision variable. Shaded cells indicate whether a particular function should be re-evaluated upon the change in a particular variable value.

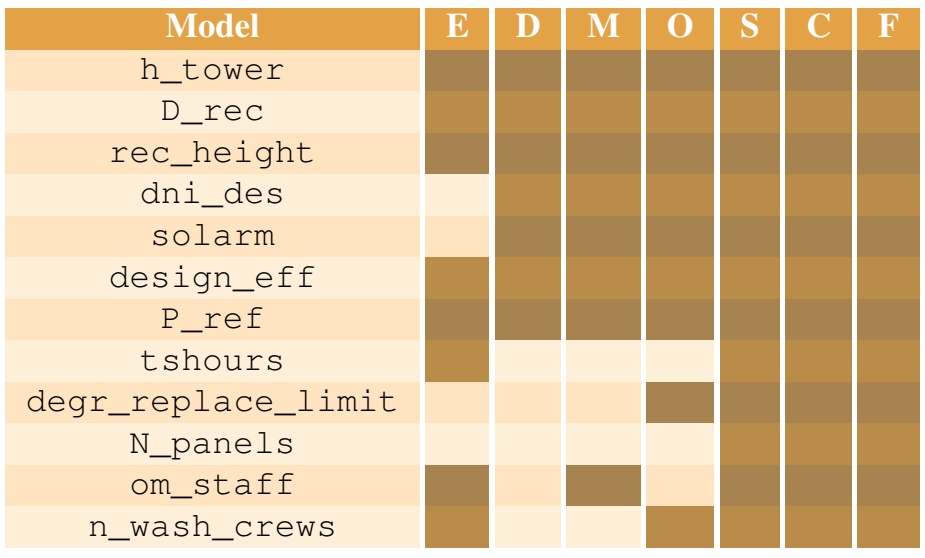

\subsubsection{Objective function}

The objective function, $f(x)$, that we minimize includes system costs, maintenance costs, and (inverse) electricity sales. We let $X$ denote the set of points that satisfy the constraints, including integrality restrictions. In practice, these constraints are bounds on the decision variables, $X$. We assume that any simulation constraints are included in the objective terms, though we could handle more general simulation constraints explicitly. Thus, our abstract model is formally stated as shown in Eq. 3.1 where the expectation, $\mathbb{E}[\cdot, \xi]$, is taken over the random parameters, $\xi$, that include stochastic parameters such as cloud cover and electricity prices. We note that we reserve the term "variable" for decision variables, and instead refer to the "random variables" $\xi$ as "random parameters" in order to emphasize the fact that we do not optimize over these random parameters.

$$
\begin{aligned}
\underset{x}{\operatorname{minimize}} f(x)=Z= & \mathcal{F}\left(\mathcal{E}\left(\bar{x}^{\mathcal{E}}\right)+f\left(\mathcal{D}\left(\bar{x}^{\mathcal{D}}\right)\right)+\mathbb{E}\left(f\left(\mathcal{M}\left(\bar{x}^{\mathcal{M}}, \xi\right)\right)\right)+\mathbb{E}\left(f\left(\mathcal{O}\left(\bar{x}^{\mathcal{O}}, \xi\right)\right)\right)\right. \\
& \left.-\mathbb{E}\left[f\left(\mathcal{S}\left(\bar{x}^{\mathcal{S}}, \mathcal{M}\left(\bar{x}^{\mathcal{M}}, \xi\right), \mathcal{O}\left(\bar{x}^{\mathcal{O}}, \xi\right), \mathcal{C}\left(\bar{x}^{\mathcal{C}}, \xi\right)\right)\right)\right]\right)
\end{aligned}
$$

Figure 3 depicts the flow of decision variables to the five models that evaluate the objective function terms.

1. The Explicit terms model $(\mathcal{E})$ includes terms whose relationship with the objective function can be written explicitly without invoking any black-box model. Examples include tower cost, receiver cost, and O\&M labor costs. The explicit model $(\mathcal{E})$ uses the variables in $\bar{x}^{\mathcal{E}}$ to calculate the explicit terms within the objective function. Tower, Receiver, TES cost, among others, are explicit relationships based on a reference concentrating solar power system developed by NREL for use in SAM. Tower costs depend on tower height and receiver 


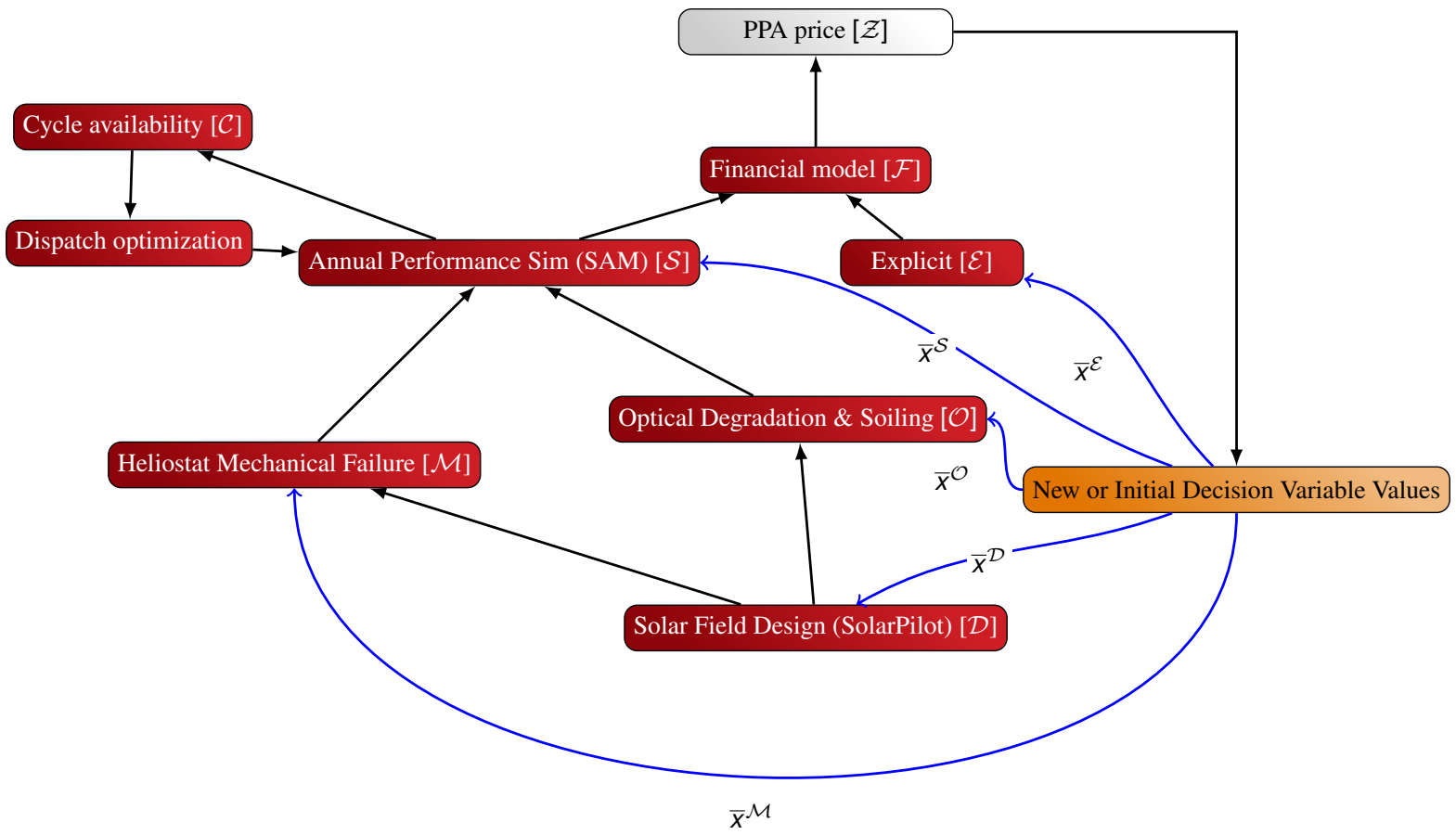

Figure 3. Variable set flow diagram

costs depend on the size of the receiver. Similarly, TES costs depend on the hours of storage, cycle design point power output, and cycle design efficiency.

2. The Solar field design and characterization model $(\mathcal{D})$ is a deterministic black-box model that utilizes SolarPILOT to create a heliostat field, assess land area requirements, and characterize optical performance. Area of the solar field, $A^{s f}$ is the total heliostat reflective surface area and is an output of the SolarPILOT design modeling capabilities. This term differs from land area of the heliostat field; however, SolarPILOT is capable of computing that area from the number of heliostats and layout of the field. Land area required for the entire CSP system, $A_{\text {land }}$, is an output of SolarPILOT and includes heliostat field area along with constant factors which account for buildings, additional land, and other required structures. After running the SolarPILOT design simulation $(\mathcal{D})$, we can use these terms in the functional forms for these costs.

3. The Heliostat mechanical failure and availability model $(\mathcal{M})$ is a stochastic black-box model that simulates heliostat failure on an hourly basis over the plant lifetime due to mechanical, electrical, or control events.

4. The Heliostat optical degradation and soiling model $(\mathcal{O})$ is a stochastic black-box model that simulates mirror soiling and optical degradation on an hourly basis over the plant lifetime. The mirror washing optimization algorithm both identifies the best procedure for washing mirrors and solves for the optimal number of wash crews given soiling rates, labor costs, etc.

5. The Annual performance simulation model $(\mathcal{S})$ is a black-box model that simulates plant electricity production, and includes the dispatch optimization sub-model that maximizes plant revenue subject to forecast uncertainty. The dispatch optimization algorithm can be posed as stochastic with respect to forecast uncertainty, although the broader $\mathcal{S}$ model is deterministic because the observed weather data itself is deterministic. This observation has significant implications for computational expense, as a deterministic $\mathcal{S}$ reduces the number of evaluations required to assess objective function improvement. 
6. The Cycle availability model $(C)$ predicts failure or performance degradation events that cause the power cycle's availability or maximum capacity to drop over a period of time until maintenance can be performed. The algorithm predicts failures using a probabilistic sampling methodology that is applied independently to different components in the cycle, where probability distribution parameters are defined based on each component's historical failure rate. The cycle availability model is called iteratively within the performance simulation in much the same way as the dispatch model is called on a rolling time horizon basis.

7. The Financial PPA price model $(\mathcal{F})$ calculates PPA price given an annual-hourly (or subhourly) power production series, system costs, an electricity sales price series, O\&M costs, and financial parameters. The financial model includes detailed cash flow analysis, tax incentives, depreciation, and other common features of utility-scale contractual arrangements. The financial model is borrowed from System Advisor Model and called via the SAM Simulation Core (SSC) application programming interface (API).

\subsection{Optimization methods}

Our optimization model possesses an objective and constraints that utilize different functional forms which describe the characteristics of, and methodology used to evaluate, a function $y=f\left(x_{1}, x_{2}, \ldots, x_{n}\right) \equiv f(\bar{x})$. The functional form $f$ is simply an operator on a set of independent variables $\bar{x}$, and may be deterministic, stochastic, externally evaluated, etc. For instance, to assess PPA price for a given scenario, including some initial cost, degradation and failure case, and operation strategy, we must employ an explicit nonlinear cost model, a "black-box" simulation to determine lifetime plant productivity, and stochastic failure and degradation models. This combination develops an accurate estimate of performance and cost and allows exploration of the impact of O\&M decisions and initial capital investment on PPA price.

Formally, the optimal design problem that we consider can be stated as a mixed-integer, derivative-free optimization problem under uncertainty:

$$
\begin{array}{ll}
\underset{\bar{x}}{\operatorname{minimize}} & \mathcal{E}(\bar{x})+\mathbb{E}[f(\mathcal{S}(\bar{x} ; \xi))] \\
\text { subject to: } & \bar{x} \in X, \quad x_{i} \in\{0,1\} \forall i \in I,
\end{array}
$$

where the components of $\bar{x}$ include both continuous and integer design variables, I indexes the set of integer variables, $\xi=\left\{\xi_{t}\right\}_{t \in \mathbb{T}}$ models the time series of uncertain parameters, $\mathcal{S}(\bar{x} ; \xi)$ is a single run of the SAM-MSPT simulation for a given design, $\bar{x}$, and a single sample path of the uncertain parameters, $\xi$, and $X$ denotes a deterministic set of constraints. This simulation is expensive to compute - typically requiring several minutes per evaluation and the black-box routine that computes $\mathcal{S}(\bar{x}, \xi)$ does not provide derivatives with respect to the design variables $\bar{x}$. The objective value for such a single realization combines the explicit objective such as investment cost and a cost that depends on the outcome of the simulation $f(\mathcal{S}(\bar{x}, \xi))$, where $\mathbb{E}[\cdot]$ is the expectation operator with respect to $\xi$, although we can easily accommodate other risk measures such as conditional value at risk, which measure resilience to a set of worst-case scenarios.

Optimal design problems such as (3.2) are challenging because they combine the combinatorial complexity of discrete decision variables with the computational challenges of simulation functions that are expensive to evaluate and for which derivatives are not available. Broadly speaking, there exist three classes of solution techniques for this class of problems: (i) evolutionary algorithms or heuristics (Goldberg); (ii) pattern-search techniques (Abramson; Audet and Dennis); and, (iii) model-based techniques (Wild, Regis, and Shoemaker; Wild). In this work, we concentrate on the latter class of algorithms, because they provide a practical approach to an a posteriori sensitivity analysis.

The three fundamental challenges of non-algebraic functions (e.g., those from SAM-MSPT), input uncertainty, and discrete decision variables imply that standard model-based mixed-integer optimization techniques (Mahajan et al.; Bonami et al.; Duran and Grossmann; Geoffrion; Westerlund et al.) cannot be applied. We develop new techniques for (3.2) that leverage existing local nonlinear solvers for derivative-free optimization that build a model of the objective function of (3.2), replacing the second term $\mathbb{E}[f(\mathcal{S}(\bar{x} ; \xi))]$ by $m_{k}(\bar{x} ; \xi)$, around a current iterate $\bar{x}_{k}$, and then compute a new iterate by solving the deterministic and fully algebraic model inside a trust-region (Wild, Regis, and 
Shoemaker; Exler and Schittkowski). Traditional derivative-free optimization techniques are designed for problems with deterministic simulations and continuous variables.

In this section, we present a new techniques for simultaneous optimization of a set of integer variables with each iterate containing a continuous optimization sub-problem. The primary goal of this work is to produce an integer problem solver that requires a sufficiently low number of model evaluations so as to ensure overall optimization problem tractability. Our approach for the solver involves constructing a set of "hyperplanes" (planes in $N$-dimensions) that together represent a surface that is guaranteed to lie below the actual model surface. The fact that the surrogate model is a valid underestimator is important in determining when the optimization algorithm has converged. When the underestimator and the actual model surface return values that are sufficiently close together, it is evident that additional optimization iterations will not be productive, and the algorithm can terminate with the "optimal" solution.

We have concentrated on three separate developments to improve the solver for the underestimator:

1. Tight bounds on the tolerance/constants, $\epsilon_{\lambda}$ and big- $M$ that are used in the mixed-integer linear programming (MILP) formulation of the master model. Tightening these bounds improves the solution time, but not to the level that makes it a competitive sub-problem solver.

2. Heuristics for selecting subsets of interpolation points that are used to create the cutting-plane model. In particular, we have defined the notion of "useful points" and developed heuristics for identifying such points. Unfortunately, we can also identify cases where it remains computationally difficult to find such points effectively.

3. Trust-region approach to accelerate the generation of the piecewise linear model. The bottleneck in our solver remains the creation of the piecewise linear solver. We can show that a trust-region approach can be developed that improves the performance without sacrificing global convergence properties.

Below, we elaborate on each of these developments in turn and show benchmark results that illustrate the solver improvement.

\subsubsection{Tight Bounds for MILP Constants.}

The MIP model leaves open the question of how to find sufficient values for model parameters called $M_{i}, \epsilon_{\lambda}$ and $M_{\lambda}$. If we choose these constants incorrectly, then the resulting MIP model no longer provides a lower bound and the optimization problem cannot be efficiently solved. Sufficient values of $M_{\lambda}$ and $\epsilon_{\lambda}$ are not straightforward to calculate, but bounds on these values can be computed by solving a set of optimization problems involving hyperplanes. Because the hyperplane is generated using $n$ integer points, it can be shown that there exists a solution $c^{i_{i j}-}$ and $b^{i_{i_{j}-}}$ that is nonzero and integral. Consider a hyperplane $S \triangleq\left\{x: c^{T} x+b=0\right\}$ such that $c$ and $b$ are integral. The Euclidean distance between an arbitrary point $\hat{x} \in \mathbb{Z}^{n} \backslash S$, and $S$, is greater than or equal to $\frac{1}{\|c\|_{2}}$. This principle can be used directly to get a sufficient value of $\epsilon_{\lambda}$ or $M_{\lambda}$, and the bounds on these parameters can be further tightened by optimization. We have also investigated "cuts" which are a way of removing unused portions of the variable space in the optimization problem, reducing the computation requirement for each iteration. 
The following techniques can be used to compute $M_{i}, \epsilon_{\lambda}$, and $M_{\lambda}$ for the MILP cutting-plane model

$$
\begin{array}{cl}
\underset{x, \lambda, z, w}{\operatorname{minimize}} & \eta \\
\text { subject to: } & \eta \geq\left(c^{i}\right)^{T} x+b^{i}-M_{j}\left(1-\sum_{j=1}^{n+1} z^{i_{j}}\right), \quad \forall i \in W(X) \\
& \sum_{j=1}^{n+1} z^{i_{j}} \leq 1, \forall i \in W(X) \\
& x=x^{i_{j}}+\sum_{\substack{l=1 \\
l \neq j}}^{n+1} \lambda_{l}^{i_{j}}\left(x^{i_{j}}-x^{i_{l}}\right), \quad \forall i \in W(X), \forall i_{j} \in i \\
& \lambda_{l}^{i_{j}} \geq-M_{\lambda}\left(1-z^{i_{j}}\right), \quad \forall i \in W(X), \forall i_{j}, i_{l} \in i, j \neq l \\
& \lambda_{l}^{i_{j}} \leq-\epsilon_{\lambda}+M_{\lambda} w_{l}^{i_{j}}, \quad \forall i \in W(X), \forall i_{j}, i_{l} \in i, j \neq l \\
& n z^{i_{j}} \leq \sum_{\substack{l=1 \\
l \neq 1}}^{n_{1}} w_{l}^{i_{j}} \leq n-1+z^{i_{j}} \quad \forall i \in W(X), \forall i_{j} \in i \\
& w_{l}^{i_{j}}, z^{i_{j}} \in\{0,1\}, \quad \forall I, j \in\{1, \ldots, n+1\}, I \neq j ; \quad \forall i \in W(X) \\
& x \in \Omega .
\end{array}
$$

The MIP model (3.2.1) leaves open the question of how to find sufficient values for $M_{i}, \epsilon_{\lambda}$ and $M_{\lambda}$. We next show that if we choose these constants incorrectly, then the resulting MIP model no longer provides a lower bound. In the remainder of this section, we indicate how to choose suitable values that guarantee the lower bounding property of this model.

A large $\epsilon_{\lambda}$ or small $M_{\lambda}$ (or both) could result in an incorrect value of $z^{i_{j}}=1$ for a point $x \notin$ cone $\left(x^{i_{j}}-X^{i}\right)$,

violating the implication of $z^{i_{j}}=1$ and yielding an invalid lower bound on $f$. Similarly, one can encounter an invalid lower bound at an iteration if $M_{\lambda}$ is chosen to be smaller than required.

We first show how to compute bounds on $M_{j}$. Let $I_{f}$ be a valid lower bound of $f$ on $\Omega$. With this lower bound, the model parameters $M_{i}$ can be defined as

$$
M_{i}=\max _{x \in \Omega}\left\{\left(c^{i}\right)^{T} x+b^{i}\right\}-l_{f}
$$

For example, if $\Omega \subset\left\{x: I_{x} \leq x^{k} \leq u_{x}\right\}$, we can set

$$
M_{i}=\sum_{h: c_{j}^{i}<0} c_{h}^{i} l_{x}+\sum_{h: c_{j}^{i}>=0} c_{h}^{i} u_{x}+b^{i}-l_{f}, \quad h=1, \ldots, n ; \forall i .
$$

Sufficient values of $M_{\lambda}$ and $\epsilon_{\lambda}$, however, are not as straightforward to calculate as they depend on the rays generated at $x^{i_{j}}$, and the domain $\Omega$. We show next, that bounds on these values can be computed by solving a set of optimization problems.

Next, we develop bounds on $\epsilon_{\lambda}$ and $M_{\lambda}$. First, we propose a sufficient value for $\epsilon_{\lambda}$ based on the representations of the $n+1$ hyperplanes formed using different combinations of $n$ points, $\forall i \in W(X)$. We can use one of the representations of the hyperplane passing through points $X^{i} \backslash\left\{x^{i_{j}}\right\}, j=1 \ldots n+1$ to obtain bounds on $\epsilon_{\lambda}$, by solving an optimization problem for each $i_{j} \in i$. We denote by $c^{i_{i_{j}}-}$ and $b^{i_{i^{-}}}$the solution of

$$
\begin{array}{ll}
\underset{c, b}{\operatorname{minimize}} & \|c\|_{1} \\
\text { subject to: } & (c)^{T} x^{i_{I}}+b=0, \quad l=1, \ldots, n+1, I \neq j \\
& \|c\|_{1} \geq 1 \\
& c \in \mathbb{Z}^{n}, b \in \mathbb{Z} .
\end{array}
$$


Problem (P-hyp) can be easily cast as an integer program by replacing $\|c\|_{1}$ by $e^{T} y$, where $e=(1, \ldots, 1)$ is the vector or all ones, and the variables $y$ satisfy the constraints $y \geq c, y \geq-c$. Because, the hyperplane is generated using $n$ integer points, it can be shown that there exists a solution $c^{i_{i_{j-}-}}$ and $b^{i_{i_{j}-}}$ that is nonzero and integral. We can now prove the following proposition.

Proposition 3.2.1 Consider a hyperplane $S \triangleq\left\{x: c^{T} x+b=0\right\}$ such that $c$ and $b$ are integral. The Euclidean distance between an arbitrary point $\hat{x} \in \mathbb{Z}^{n} \backslash S$, and $S$, is greater than or equal to $\frac{1}{\|c\|_{2}}$.

Proposition 3.2.1 can be used directly to get a sufficient value of $\epsilon_{\lambda}$ as follows.

$$
\epsilon_{\lambda}=\min _{i \in W(X), i_{j} \in i} \frac{1}{\left\|c^{i_{i_{j}-}}\right\|_{2}} .
$$

The bound can be tightened using the following optimization problem.

$$
\begin{array}{cl}
\underset{x}{\operatorname{minimize}} & \frac{\left|\left(c^{i_{i_{j}-}}\right)^{T} x+b^{i_{i_{j}-}}\right|}{\| i_{i_{j-}}} \\
\text { subject to: } & \left|\left(c^{i_{i_{j}-}}\right)^{T} x^{i_{l}}+b^{i_{i_{j}-}}\right| \geq 1 \\
& x \in \Omega .
\end{array}
$$

If we denote by $\epsilon_{\lambda}^{i_{j_{j}-}}$, the optimal value of $(\mathrm{P}-\epsilon)$, then the following is a sufficient value of $\epsilon_{\lambda}$.

$$
\epsilon_{\lambda}=\min _{i \in W(X), i_{j} \in i} \epsilon_{\lambda}^{i_{i_{j}-}}
$$

Similarly, if we maximize the objective in $(\mathrm{P}-\epsilon)$, and denote the optimal value by $M_{\lambda}^{i_{i_{j}-}}$ we get a sufficient value for $M_{\lambda}$ as follows.

$$
M_{\lambda}=\max _{i \in W(X), i_{j} \in i} M_{\lambda}^{i_{i_{j}-}}
$$

It is also possible to update the values of $M_{i}, \epsilon_{\lambda}$, and $M^{\lambda}$ before solving (3.2.1) at each iteration.

We have also investigated so-called "no-good-cuts". The problem with having values of $\epsilon_{\lambda}>0$ too small is that it allows us to ignore the cuts at the interpolation points, $x^{(k)}$, causing a violation of the bound, $\eta \geq f^{(k)}$, for $x=x^{(k)}$ in the MIP problem resulting in repetition of iterates in the algorithm. We can easily fix this situation, either with "no-good cuts", which exclude a single integer assignment, or by adding the following valid inequality to the MIP:

$$
\eta \geq f^{(k)}-M\left\|x^{(k)}-x\right\|_{1},
$$

which we can write as a linear constraint, if we introduce a binary representation of the variables, $x$, requiring $n U$ binary variables, $\xi_{i j}, i=1, \ldots, n, j=1, \ldots, U$, where $n$ is the dimension of our problem, and $L$ is the upper bound on $0 \leq x \leq U$. With this representation, we obtain

$$
x_{i}=\sum_{j=1}^{U} i \xi_{i j}, \quad 1=\sum_{j=1}^{U} \xi_{i j}, \quad \xi_{i j} \in\{0,1\},
$$

and write the $\eta$-constraint equivalently as

$$
\eta \geq f^{(k)}-M \sum_{i=1}^{n}\left(\sum_{j: \xi_{i j}^{(k)}=0} \xi_{i j}+\sum_{j: \xi_{i j}^{(k)}=1}\left(1-\xi_{i j}\right)\right) .
$$


We can also spawn fewer binary variables using the following.

$$
x_{i}=\sum_{j=1}^{\left\lfloor U_{i}\right\rfloor} 2^{j} \cdot \xi_{i j}, \quad \xi_{i j} \in\{0,1\},
$$

\subsubsection{Heuristics for Accelerating the Creation of Cutting Planes}

We have observed that the number of cut for our piecewise linear model grows exponentially with the iteration count. In order to keep the work per iteration manageable, we have investigated the notion of useful points, which are points that generate cuts that exclude other non-optimal points.

We start by defining what it means for a point $\bar{x} \in \Omega$ to be "necessary". A point is necessary, if no poised set of $n+1$ points, $X^{i} \subseteq \Omega$, exists such that $\bar{x} \in U^{i}$ and $f\left(x^{*}\right) \leq c^{i^{T}} \bar{x}+b^{i}$. It is obvious that $\bar{x}$ will have to be evaluated (irrespective of the search strategy) and must belong to every optimal set $X^{*}$, sufficient for convergence of our Algorithm. Determining the set of necessary points does not seem viable without realizing the values of $f$ over entire $\Omega$. It is noteworthy that at any iteration, the set of evaluated points is typically a strict subset of $\Omega$.

Similarly, we can define the notion of useful cuts. Figure 4 show the number of "useful" cuts generated per iteration using the 'lowest function value' strategy for 3 test functions on the grid $[-4,4]$ for $n=3,4$, when all possible cuts are generated at each iteration. A cut is termed 'useful' at an iteration $k$ if it prunes at least one 'promising' point $x \in P^{k}$ at an iteration $k$, where $P^{k} \triangleq\left\{x \in \Omega: \eta^{k-1}(x)<u_{f}^{k-1}\right\}$.
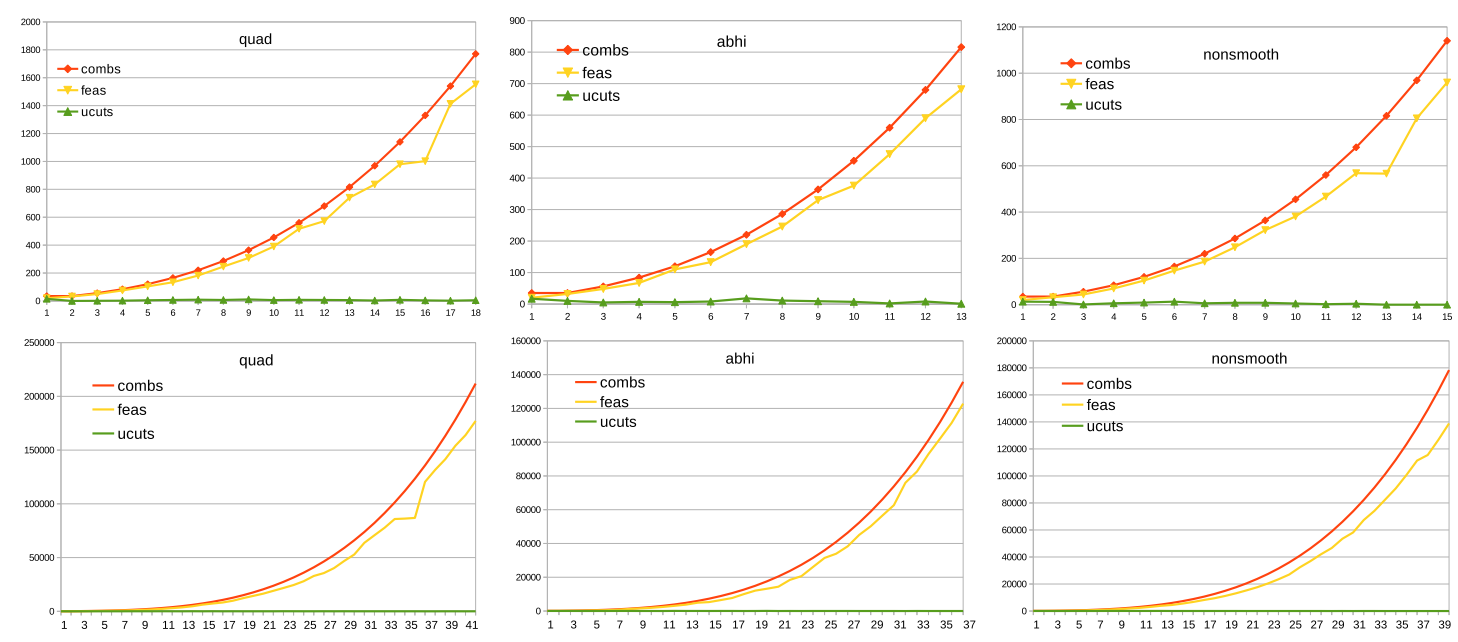

Figure 4. Graphs showing the number of feasible combinations of $n+1$ points and the secants that pruned at least one 'potential' point at an iteration for $n=3$ and $n=4$.

Similarly, Figure 5 shows the number of 'useful' interpolation points corresponding to Figure 4 . A point is termed 'useful' at an iteration $k$ if it constitutes at least one useful cut. An important observation is that though the fraction of useful cuts is typically very small, the fraction of useful points could be high, indicating that most of the available interpolation points may contribute at least one useful cut.

\subsubsection{New Trust-Region Method for Integer Optimization}

To further improve the efficiency of our approach, we have developed trust-region approach. The basic idea is to consider the cutting-plane model inside an $\ell_{\infty}$-norm trust region, to reduce the number of points that must be considered in the cutting-plane model. If we make progress and reduce the objective function, then we move the trust-region to our new iterate. Otherwise, we continue to generate new points within the trust-region. Once the trust-region model appears to indicate optimality, we must increase its radius to avoid getting trapped in local minimizers. 

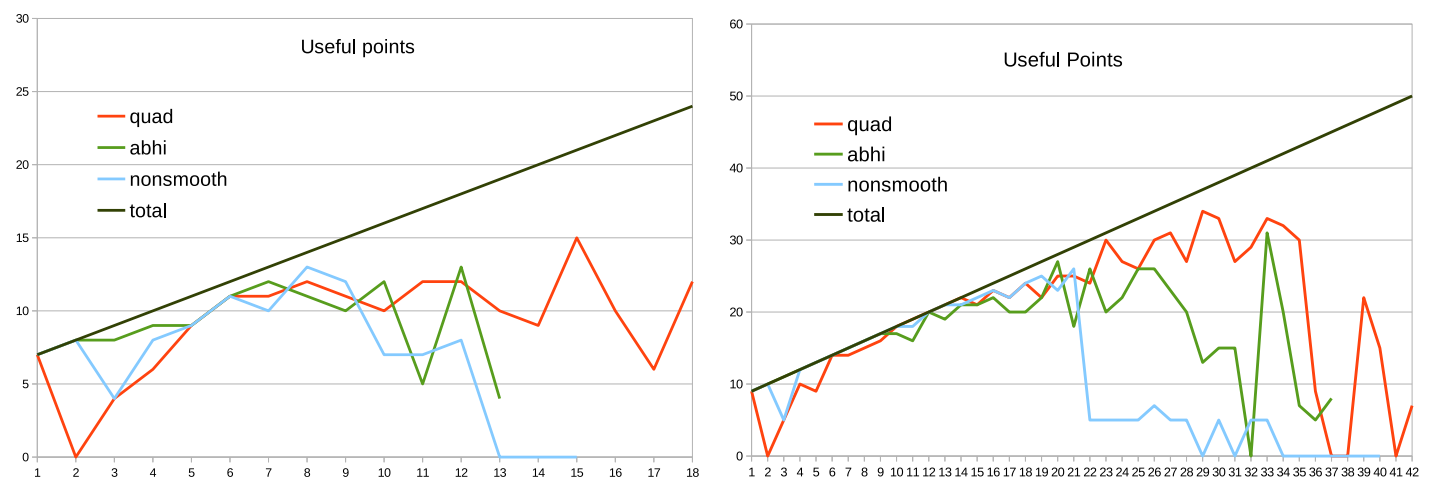

Figure 5. Graphs showing the number of points that formed 'useful' secants, those which pruned at least one 'potential' point at an iteration for $n=3$ and $n=4$.

We start by describing the master problem that encodes the conditions in which each secant hyperplane is a valid underestimator of $f$. Let $W(X)$ denote the set of all multi-indices $i$ corresponding to poised subsets $X^{i}$ of $X$. If $f$ has been evaluated at every point in $X$, we construct the secant hyperplane $\left(c^{i}\right)^{T} x+b^{i}$ for every multi-index $i \in W(X)$.

$$
\begin{array}{ll}
\underset{x}{\operatorname{minimize}} & \eta \\
\text { subject to: } & \eta \geq\left(c^{i}\right)^{T} x+b^{i}, \text { if } x \in U^{i}, \forall i \in W(X) \\
& \eta<U, x \in \Omega,
\end{array}
$$

where $U$ is a current upper bound. This problem can be formulated as a MILP, however, it is more efficient to instead update the values of $\eta$ as we add new cuts to our model, using the heuristics described in the previous section.

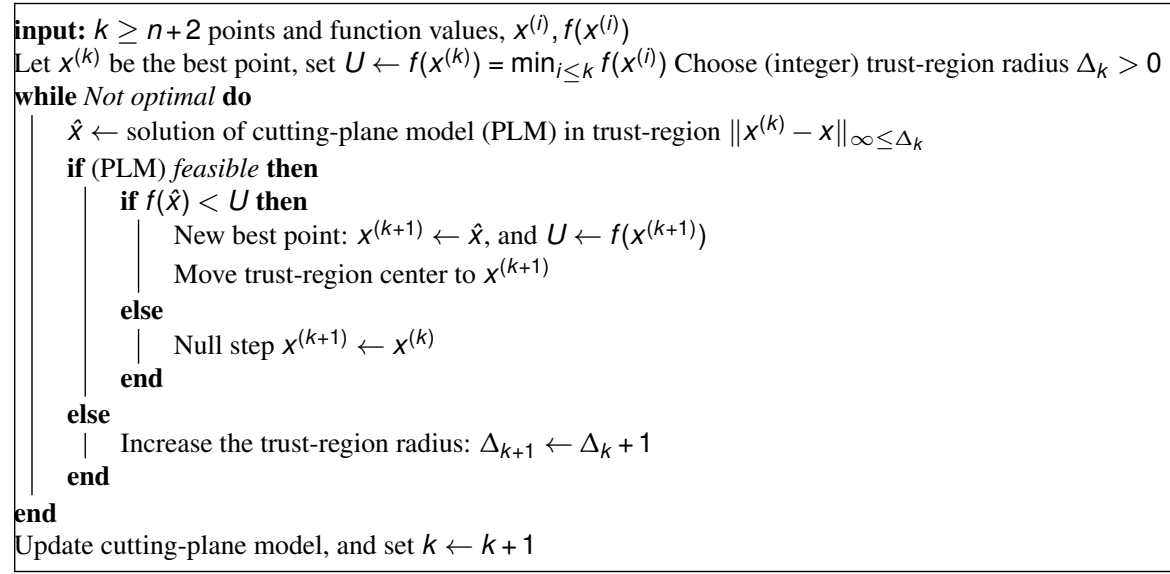

\section{Algorithm 1: Trust-Region Algorithm for Solving MIP-DFO}

We note that the increase in trust-region radius is only activated when the cutting-plane model PLM becomes infeasible. This update ensures that the algorithm remains globally convergent.

\subsubsection{Performance Improvements}

Overall, the heuristics described in the previous sections greatly improve the effectiveness of our mixed-integer algorithm, and enables us to solve instances with more integer variables to global optimality. Figure 6 shows the improvement on a benchmark problem for solving instances of size $n=2,3,4$ that resulted from the new trust-region approach. The solid lines indicate the convergence of the optimality gap without the trust-region, and the dashed lines the convergence with the trust region. 

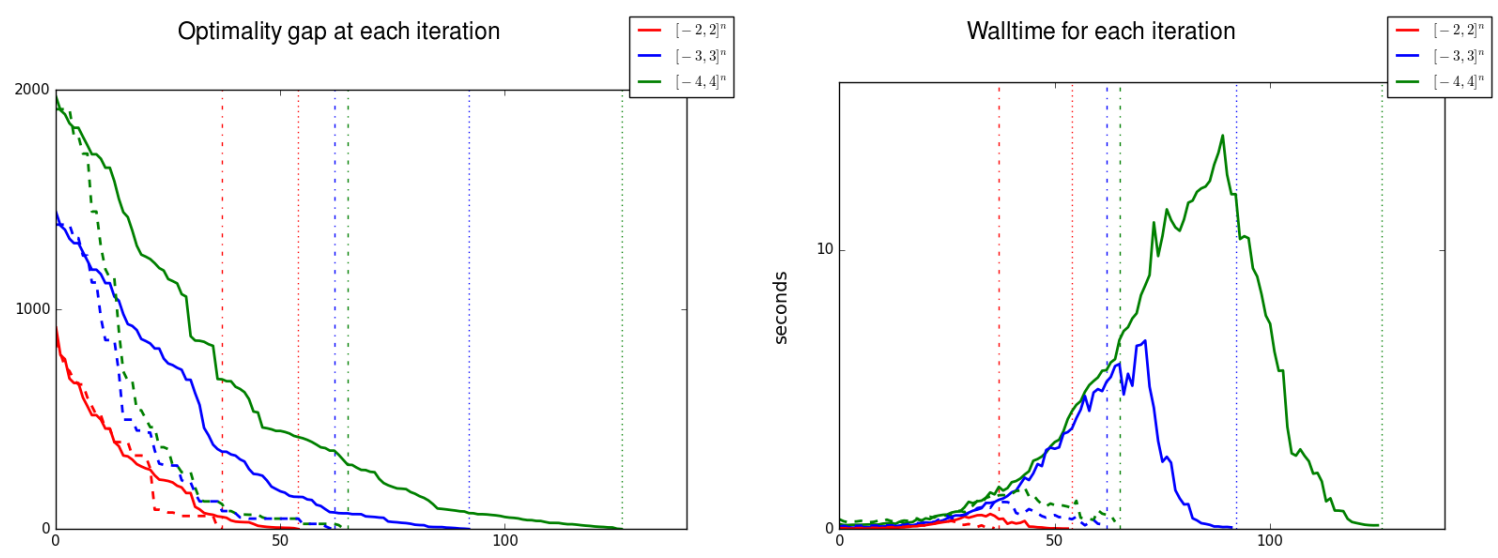

Figure 6. Convergence of optimality gap (left) and wall-clock time (right) for trust-region (dashed) versus traditional approach (solid),

The overall improvement in the heuristics is illustrated also in Figure 7. Blue and red bars show the MIP model using CPLEX, which time out for problems of dimension $n=3$, yellow is the enumeration scheme which times out for dimension $n=4$, and green and brown bars correspond to the enumeration scheme with heuristics and trust region added respectively, which enable us to solve slightly larger problems.

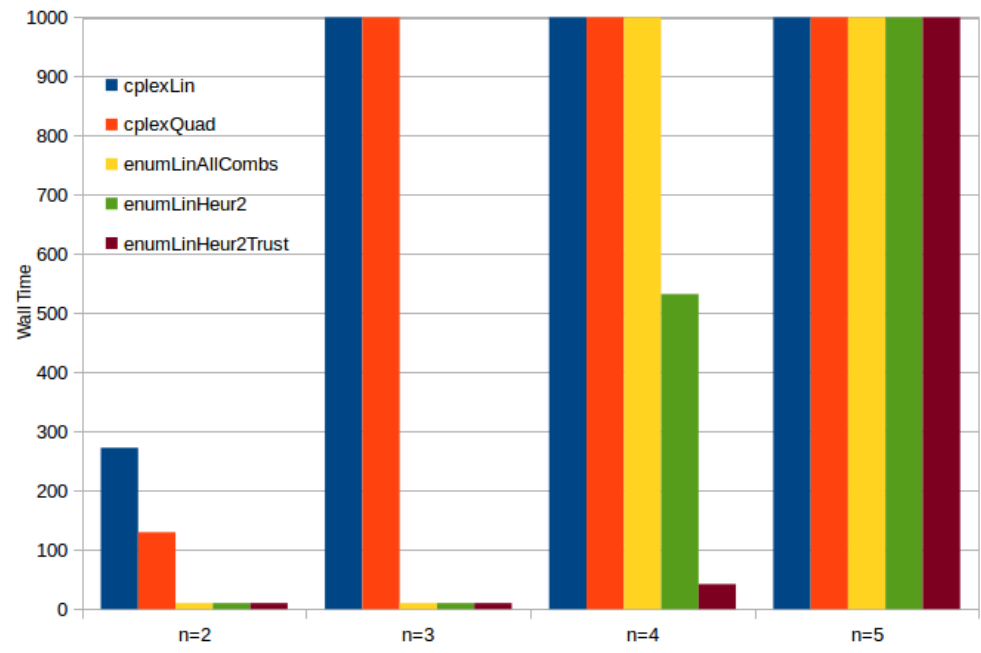

Figure 7. Improvement in wall-clock solution time with different cutting plane models. Blue and red bars show the MIP model using CPLEX, the yellow bars are the simple enumeration scheme, and the green and brown bars correspond to the enumeration scheme with heuristics and with trust region added.

\subsubsection{Solver Implementation Summary}

Much of the work regarding solver development has emphasized techniques for solving problems involving integer variables, but the fact remains that the majority of the variables involved in design and operations decisions can be expressed as continuous, at least from a predictive modeling standpoint. With this in mind, we have now combined the integer solver with a nonlinear solver for the continuous sub-problem. In essence, as the integer solver determines the response surface and direction of descent, each point of evaluation for the integer variables involves a full optimization of the continuous sub-problem. This approach involves somewhat more computational expense than a 
purely continuous optimization problem, but the ability to optimize both non-relaxable integer and standard continuous variables in a black-box, nonlinear problem is a significant advance. We note that the continuous sub-problem can be solved by conventional nonlinear solvers, of which several freely available options are suitable. Our solution involves pairing the novel integer variable optimization program with NLOpt's BOBYQA and/or COBYLA algorithms; these have previously been used with some success by the team for system design, heat exchanger sizing, and other applications.

\subsection{Probabilistic predictive modeling}

The integration of intermittent renewable sources into the power grid, without impacting system reliability and without increasing costs, poses challenges. Mitigating the risk of uncertain solar resource availability can significantly increase the economic viability of plants relying on solar energy (Kaur, Pedro, and Coimbra). Accurate forecasts of solar irradiance is central to achieving substantial economic benefit. Due to the pervasive use of PV technology, forecasting global horizontal irradiance (GHI) is a relatively mature area. In contrast, our CSP technology requires forecasts of DNI, and accurately forecasting DNI is significantly more difficult than GHI.

A CSP system with TES helps mitigate some of the risk associated with uncertain solar irradiance. That said, fully exploiting CSP systems requires dispatch optimization coupled with accurate forecasts of both DNI and uncertain energy prices. The economic analysis of storage systems in CSP in different markets has received some attention, e.g., (Kost, Flath, and Möst; Usaola; Lizarraga-Garcia et al.). With TES, CSP has the capability of dispatching energy when energy prices are high, and holding thermal storage when prices are relatively low. Again, the importance of accurate DNI forecasting, coupled with accurate price forecasting is paramount. If ample DNI will be available tomorrow, our system can produce power aggressively today, while if solar irradiance will be low tomorrow, then we should maintain enough storage at the end of today to capture peak energy prices tomorrow. Of course, DNI and prices cannot be forecast perfectly, and hence capturing the probability of high prices coupled with low DNI availability is key to dispatching the system in a manner that is well-hedged against a collection of DNI and price scenarios.

We address the following issues with respect the uncertain operating environment of a CSP plant:

1. Characterizing the uncertainty in solar irradiance and electricity prices, using predictors rooted in both historical data and in weather forecasts.

2. Developing a dispatch optimization model that takes as input up-to-date forecasts and takes advantage of the nature of thermal energy storage.

\section{Uncertainty in DNI and Energy Prices}

We characterize the uncertainty in both DNI and energy prices using autoregressive time series models coupled with exogenous predictors. Time series models attempt to quantify the significant autocorrelation between the variable of interest and the historical data; see, e.g., (Tsay). Here, autocorrelation refers to the dependency structure that occurs between a random variable such as DNI in one time period (e.g., hour) and the same random variable in a previous time period. So, DNI in hour $t$ can be used to help predict DNI in a future hour $t^{\prime}>t$, effectively building a regression model that uses the former as a predictor of the latter. Time series models can also account for temporal variability via time-dependent coefficients, differencing, extracting seasonality, etc. In the literature on forecasting DNI, several time series models have been put forward, including autoregressive models (AR), autoregressive moving-average models (ARMA), variants of these models that use additional exogenous predictors, and other models such as artificial neural networks and support vector machines; see, e.g., Marquez and Coimbra; Reikard; Law, Kay, and Taylor; Mellit et al.

Broadly speaking, two important classes for estimating energy prices include: (i) models rooted in stochastic processes such as the mean-reverting Ornstein-Uhlenbeck process, and (ii) time series and regression approaches similar to those just sketched. In the former approach, the mean reversion property is desirable because if we were to instead assume a model of, say, geometric Brownian motion, then after a jump, energy prices would "re-center" at the new level, and this is inconsistent with energy price data. For examples of the former approach see, e.g., Blanco and 
Soronow. While we investigated using an Ornstein-Uhlenbeck process, we found that it did not provide an adequate fit, and so we do not report on it in detail here. Reference Tsay includes a good overview of the latter approach to forecasting energy prices using time series models.

In what follows, we use autoregressive moving average time series models with exogenous predictors, which we refer to as ARMA-X models, to fit both DNI and price data. We analyze the correlation between DNI and price, and how this dependency plays a role in our predictive models.

Overall, our DNI forecasting work studied four data sets: (i) TMY3 data for the site Blythe Riverside Airport, California; (ii) SURFace RADiation (SURFRAD) data for Desert Rock, Nevada (DRA); (iii) SOLRAD (formerly ISIS) data for Albuquerque, New Mexico (ABQ); and, (iv) SOLRAD data for Hanford, California (HNX). Relative to (i), data sets (ii)-(iv) have the advantage of being historical data for multiple years at one-minute or three-minute resolution, depending on the specific year, rather than a typical year of composite data with coarser hourly resolution. There is a lack of price data available at sites (ii) and (iii), but we do have price data available from the California Independent System Operator (CAISO) near Hanford, at least for recent years. In what follows we report results for both (iii) and (iv), but when we need to use both price and DNI simultaneously we focus on Hanford.

\subsubsection{Solar resource forecasting}

Global horizontal irradiance, i.e., total solar irradiance, is composed of DNI and diffuse horizontal irradiance (DHI), and ground-reflected radiation, although the latter-most type is ignored in practice as being insignificant compared to DNI and DHI. As discussed above, CSP plants generate electricity via the DNI component of solar irradiance. While some work in the literature attempts to forecast DNI using a GHI forecast, this is not preferred in the context of CSP because GHI and DNI are affected differently by various weather conditions (Law et al.). For these reasons, we prefer to directly forecast DNI.

DNI data follow a diurnal cycle and exhibit nonstationary variability, meaning that the variance of DNI cannot be characterized with a single value but rather changes over time (e.g., both with the time of day and with the month of the year). We use ARMA models with additional predictors from the National Digital Forecast Database (NDFD) of the National Weather Service (NWS), and our approach is motivated in large part by the work of Marquez and Coimbra [41], although we employ simpler forecasting models than their neural networks.

\section{DNI data aggregation}

The observed DNI values are recorded at a much finer time resolution-one-minute and three-minute incrementsthan the hourly dispatch decisions that are employed in our optimization model. For this reason, we aggregate these data to coarser time scales prior to performing a statistical fit. A simple approach is to aggregate data at, say, onehour or thirty-minute resolution. However, this simple approach has some drawbacks because the initial, and final, time periods in each day will have fewer positive DNI observations due to the specific time of sunrise and sunset. Through numerical experimentation, we observed that using time periods that are instead of equal length between sunrise and sunset significantly improves forecasting accuracy. In particular, we select a number of bins for a day, say $B$. Then, putting aside rounding issues, if we have a total of $N$ minute-level observations of DNI for a specific day, the first bin contains the first $N / B$ observations, the second bin contains the second $N / B$ observations, etc. Thus, if $B=10$, which is what we use in numerical results reported below, each bin contains $10 \%$ of the daylighthour observations of DNI. With this approach, the temporal duration of one bin slowly changes over the course of the year.

\section{DNI data transformation}

An ARMA model assumes stationarity of the data, meaning that the data points fluctuate around a fixed mean with a constant variation. In contrast, the DNI data are seasonal because the sun's position changes in the sky over the course of a day and over the course of a year. We remove this seasonality by subtracting an estimate of the timevarying mean and dividing by an estimate of the time-varying standard deviation. A straightforward application of this idea, based on the hour of the day (e.g., the 10am hour), fails to achieve the desired goal because daylight hours vary over the course of the year. So, consistent with the bin-based idea discussed above, we introduce a parameter, 
denoted $\lambda(t)$, where $t$ corresponds to a time at which clearsky is positive (a daylight hour) and $\lambda(t)$ measures the proportion of the daylight hours that have elapsed on a given day:

$$
\lambda(t)=\frac{t-t_{r}}{t_{s}-t_{r}}
$$

Here, $t_{r}$ and $t_{s}$ denote the sunrise and sunset times of the day. Note that $0 \leq \lambda(t) \leq 1$ for time $t$ for which the clearsky value is positive. Throughout we use the clearsky model of Ineichen and Perez; Perez et al.

We use a combination of two methods for transforming the DNI dataset in order to remove seasonality and obtain (near) stationarity. Both of our methods effectively equate distinct times $t$ and $t^{\prime}$ when they have equal values of $\lambda$, i.e., when $\lambda(t)=\lambda\left(t^{\prime}\right)$ : (i) we standardize DNI at time $t$ by the moving average (denoted $\mu_{M A}(t)$ ) and standard deviation (denoted $\sigma_{M A}(t)$ ) of the last 30 days, and (ii) we standardize DNI at time $t$ by the average (denoted $\mu_{P Y}(t)$ ) and standard deviation (denoted $\sigma_{P Y}(t)$ ) of the same month in the previous year. The method we use employs a weighted combination of these two ideas using weight $0<w_{M A}<1$ :

$$
\begin{gathered}
\mu_{W C}(t)=w_{M A} \cdot \mu_{M A}(t)+\left(1-w_{M A}\right) \cdot \mu_{P Y}(t) \\
\sigma_{W C}^{2}(t)=w_{M A} \cdot \sigma_{M A}^{2}(t)+\left(1-w_{M A}\right) \cdot \sigma_{P Y}^{2}(t) .
\end{gathered}
$$

We calculate the standardized DNI value, denoted by $\widetilde{D N} I_{t}$, as

$$
\widetilde{D N} I_{t}=\frac{D N I_{t}-\mu_{W C}}{\sigma_{W C}} .
$$

The standardized DNI may exhibit extreme values that limit our ability to forecast well. To this end, we apply a transformation that truncates standardized values outside of a threshold value, which we denote $\gamma$. A typical such truncation (see, e.g., Uniejewski, Weron, Ziel, et al.) produces values as:

$$
Z_{t}= \begin{cases}\gamma \operatorname{sgn}\left(Y_{t}\right), & \text { if }\left|Y_{t}\right|>\gamma \\ Y_{t}, & \text { if }\left|Y_{t}\right| \leq \gamma\end{cases}
$$

where $\operatorname{sgn}(\cdot)$ is the sign of its argument. Thus, if $\gamma=3$ then we truncate values that deviate from the mean by more than three standard deviations. It is also possible to truncate values in a one-sided manner, meaning the upper or lower tail. We apply one-sided truncation with $\gamma=3.2$ on the lower tail. The motivation for this is that, for our purposes, we do not want low values of DNI, which are unimportant for operating a CSP system well, to distort the fit.

\section{Exogenous variables for DNI fitting}

Exogenous variables provide information that is not inherent within the time series under consideration. We consider a set of candidate exogenous variables for our ARMA-X models, similar to those NWS NDFD predictors used in the study of Marquez and Coimbra, with additional clearsky and $C S \cdot(1-S C)$ predictors, which is the product of the clearsky (CS) and the complement of the sky-cover (SC) value; see Nonnenmacher, Kaur, and Coimbra. Table 3 lists the exogenous variables we investigated.

We normalize the exogenous variables so that their values lie between 0 and 1 , by subtracting the minimum value and then dividing by the range. We access historical forecast values of these exogenous variables through the NDFD database, which consists of gridded digital forecasts of these weather elements for 16 predefined geographical sectors throughout the U.S. The NDFD provides forecasts of weather elements up to a resolution of $2.5-\mathrm{km}$ spatially and 1-hour temporally. In addition to values forecast for future, the NDFD also provides historical forecast values for predefined sectors. The software allows us to probe the database at a specific latitude and longitude and for a specific weather element. With this functionality, we have access to NDFD database for specific weather elements, e.g., sky cover, and for a location close by any station we consider in our numerical experiments. 


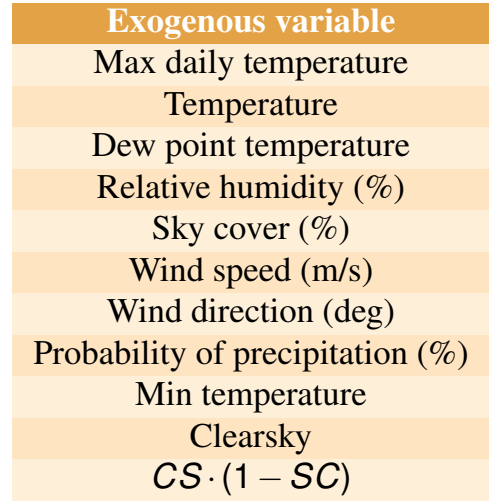

Table 3. Candidate exogenous variables.

of these using observed data.

\section{DNI model}

Using the standardized and transformed DNI values, we fit an ARMA-X model:

$$
\begin{aligned}
Z_{t}= & \phi_{0}+\phi_{1} Z_{t-1}+\cdots+\phi_{p} Z_{t-p} \\
& +\theta_{1} \varepsilon_{t-1}+\cdots+\theta_{q} \varepsilon_{t-q} \\
& +\beta_{1} X_{1, t}+\cdots+\beta_{r} X_{r, t}+\varepsilon_{t}
\end{aligned}
$$

where $\left\{\varepsilon_{t}\right\}$ is a sequence of independent and identically distributed mean-zero normal random variables; $p$ and $q$ are nonnegative integers; $\phi_{0}, \phi_{1}, \ldots, \phi_{p}$ and $\theta_{1}, \ldots, \theta_{q}$ are autoregressive and moving-average parameters, respectively; and, $X_{1, t}, \ldots, X_{r, t}$ are exogenous predictors with parameters $\beta_{1}, \ldots, \beta_{r}$. We estimate all

\section{DNI fitting results}

We compute the quality of models based on goodness-of-fit measures between our predictions and observed data using mean absolute error (MAE), square of the correlation coefficient $\left(R^{2}\right)$, and root mean square error (RMSE). We partition the data into a training dataset and a testing dataset. In assessing goodness of fit, we "roll forward" on the test dataset with six-hour time increments, and compute the goodness-of-fit measures on these six-hour-ahead forecasts. In order to select the specific model from the family of models described by equation (3.11), we perform a stepwise analysis, analogous to stepwise regression. At each step of the stepwise analysis, we greedily choose the predictor for which the improvement in goodness-of-fit measures is the largest. First we focus on selecting the $p$ and $q$ parameters that characterize the lag in the ARMA portion of the ARMA-X model, and then we choose the subset of exogenous predictors to incorporate into the model.

Based on our numerical analysis, we choose $w_{M A}=0.1$, meaning that $10 \%$ weight is put on the previous month and $90 \%$ weight on the previous year for observed DNI values. Our stepwise analysis leads to our choice of the ARMA-X(2,1) model, i.e., we use $p=2$ autoregressive terms and $q=1$ moving average term. In terms of exogenous predictors, we select sky cover, clearsky, $C S(1-S C)$, and probability of precipitation. In addition, as we roll forward on the test dataset, we refit the parameters of the model every 10 days. (We simply refit the model with respect to the predictors that have already been selected; i.e., we do not redo the stepwise analysis.)

The MAE, RMSE, and $R^{2}$ goodness-of-fit measures can be computed in-sample (on the training set) and out-ofsample (on the testing set). There is typically some degradation as we move from in-sample to out-of-sample results, due to over-fitting on the training set. Tables 3.4(a) and 3.4(b) show representative results. The MAE and RSME values degrade from 93.0 and 149.4 to 107.6 and 168.0 while the $R^{2}$ value degrades from 0.82 to 0.76 when we move from a train set to a test set. The middle columns in these tables show analogous results when only using DNI from the previous period as a predictor. Not surprisingly, the fit is poorer, but there is little-to-no degradation from in-sample to out-of-sample data. While such statistical measures provides insight regarding the quality of the ARMA-X models that we fit, the more important measure of quality in our setting is the potential for the ARMA$\mathrm{X}$ model to allow us to capture increased revenue when dispatching a CSP plant. We will explore this below, after reviewing our statistical forecasting models for energy prices.

\subsubsection{Electricity sales price forecasting}

The data we use for forecasting energy prices are available from the electricity markets in California, which are operated by CAISO, the California Independent System Operator. While there are markets for ancillary services (regulation up, regulation down, spinning reserve and non-spinning reserve), here we focus on energy markets. The CAISO energy markets produce locational marginal prices (LMP), which capture the marginal cost of serving an additional unit of demand (\$/MWh) at a particular bus in the electric network. The historical LMPs in the real-time market (RTM) and the day-ahead market (DAM) are available through a CAISO Web interface. 


\section{Table 4}

(a) Goodness of fit associated with stepwise analysis of ARMA-X model on train data (2010) for Hanford.

\begin{tabular}{|l|cc|}
\hline & $(1,0)$ & Fit A RMA-X Model \\
\hline MAE & 130.4 & 93.0 \\
$R^{2}$ & 0.72 & 0.82 \\
\hline RMSE & 183.9 & 149.4 \\
\hline
\end{tabular}

(b) Goodness of fit associated with ARMA-X model on test data (2011) for Hanford.

\begin{tabular}{|l|cc|}
\hline & $(1,0)$ & Fit A RMA-X Model \\
\hline MAE & 126.0 & 107.6 \\
$R^{2}$ & 0.71 & 0.76 \\
\hline RMSE & 183.8 & 168.0 \\
\hline
\end{tabular}

In order to download a large price dataset from this interface, we built a Web scraper, which can handle downloading and parsing price data automatically. The bulk of the energy tends to be sold in the day-ahead market, yet as we will describe further below, our numerical analysis indicates that there are significant opportunities for CSP systems in real-time markets because of its volatility; see also Dowling, Kumar, and Zavala. Therefore, we focus on using LMPs both in the DAM and RTM in our numerical experiments.

The day-ahead market (DAM) releases results at 1pm for the following day, including prices with hourly resolution. The intra-day real-time market (RTM) includes an hourly market as well as shorter-term fifteen-minute (RTM15) and five-minute (RTM5) markets. Relative to the DAM, the RTMs are more volatile processes with larger price fluctuations. The hourly RTM closes 75 minutes before the trading hour starts and opens up for bids at $1 \mathrm{pm}$ before the trading day. Market participants can buy and sell additional power in the RTM to satisfy the load not covered by day-ahead schedules. In other words, the RTM serves to mitigate the discrepancies between the realized demand and the forecasted demand. The RTM tends to be a more volatile market with larger price fluctuations.

\section{Price data transformation and aggregation}

Figure 8 shows hourly LMP DAM price data, in box-and-whisker format, for CONTADNA_1_N001 (near Hanford, California), for 2015-2017, for the months of January, April, July, and October. Like DNI data, we can see that price data exhibit seasonality within a day and differences over a the course of a year. As a result, we perform the same transformation as shown in equations (3.8) and (3.9). We do not perform a truncation of the type shown in equation (3.10) with the price data.

We face the following issue in reconciling how we forecast DNI and energy prices. In the former case, as we have sketched above, we focus on daylight hours and use time-bins that are equally spaced from sunrise to sunset. Dayahead energy prices and the hourly RTM market prices are specified in "on-the-hour" hourly format, and shorterterm RTM prices have finer time resolution. In the same way we aggregate one- or three-minute DNI values, we similarly aggregate, e.g., the 15-minute RTM prices to hourly resolution. Then for all price data, we perform all forecasts on the natural hourly basis, rather than the basis used when forecasting DNI.

\section{Price model}

The basic mathematical form of the ARMA-X model we use for forecasting energy prices is the same as that used in forecasting DNI; i.e., we use equation (3.11). The following are the candidate exogenous predictors that we use for the price model: (i) DNI observations, (ii) hourly average of price data in the last two weeks, (iii) maximum price observed yesterday, (iv) minimum price observed yesterday, (v) the price data of the subsequent hour yesterday, and (vi) ambient temperature.

\section{Price fitting results}

We follow the same procedure, i.e., stepwise analysis with goodness-of-fit measures, described when fitting the DNI model in order to select the energy price model. The procedure indicates that incorporating DNI as an exogenous predictor in the price model improves its accuracy. In addition, we include the average hourly price over the past two weeks. In terms of DNI, we note that we use our conditional DNI point forecast as the exogenous predictor when requisite DNI observations have not yet been realized. We choose $p=3$ autoregressive terms, and $q=0$ moving 


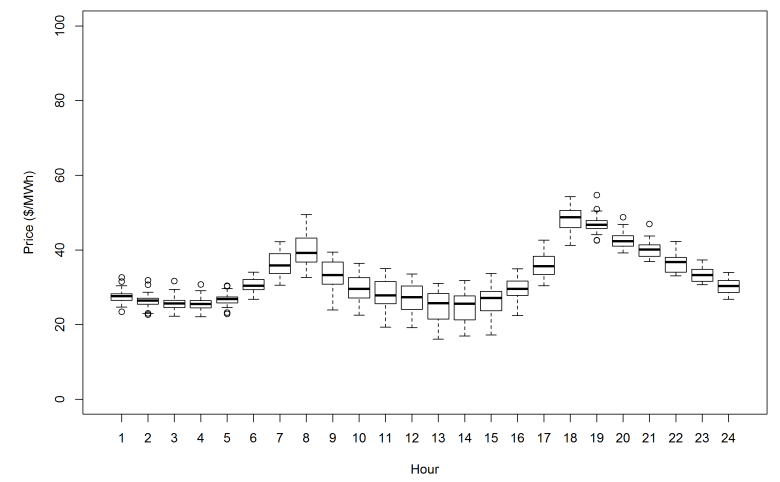

(a) January

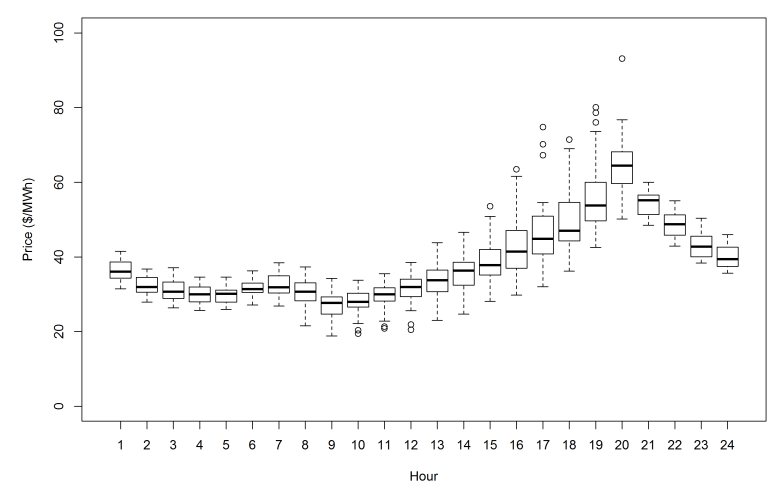

(c) July

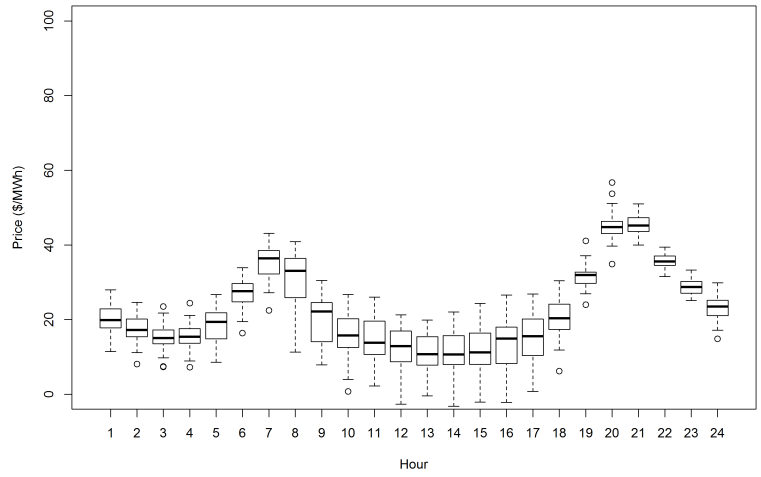

(b) April

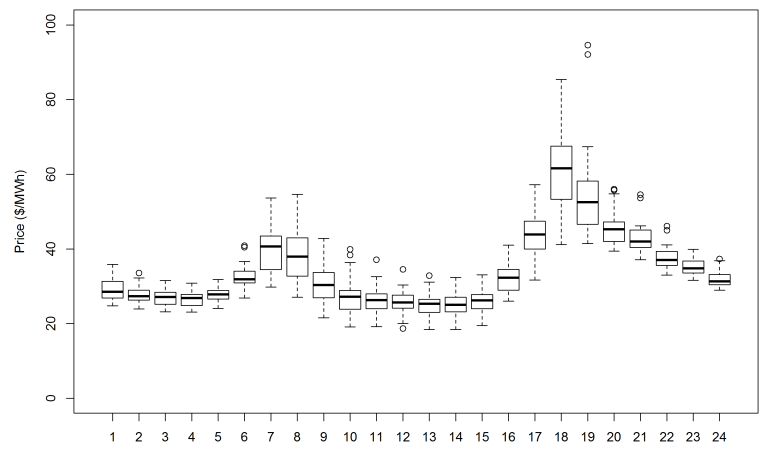

Hour

(d) October

Figure 8. Boxplots of hourly LMP DAM price data over 2015-2017 in months January, April, July, and October for node CONTADNA_1_N001.

average terms for the ARMA-X price model.

Again, MAE, RMSE, and $R^{2}$ values were used to assess the goodness of fit, and we follow the same in-sample and out-of-sample procedure sketched above for DNI. We do not report these values in detail here, and defer assessment of the value of the energy price model to the below when it is coupled with the dispatch optimization.

\subsubsection{Heliostat field availability}

The heliostat field availability model is designed to approximate the relationship between the stochastic nature of component-level failure events, the number of staff available to carry out repairs, and the proportion of heliostats that are available for energy production over time. The model simulates failures and repairs in the heliostat field by generating component lifetimes and repair times that are governed by user-specified random variables. When the lifetime of any of a heliostat's components expires, the heliostat no longer functions and enters a repair queue until an O\&M staff member is available to remove the component from the queue to perform repairs. After the component is restored, it is treated as either "good-as-new" or "good-as-old" according to user specifications, and the heliostat is once again operational until the next component failure.

To our knowledge, there is little to no publicly available data on heliostat component failures over long durations. 
Hence, our goal is to establish a model framework that is flexible enough to accept either a simple set of inputs (e.g. overall mean time-to-failure and mean time-to-repair) or a more complex set of inputs (e.g. different time-tofailure or time-to-repair distributions for many components) that may be proprietary or available in the future. The model treats each heliostat as a collection of components and/or failure mechanisms that operate serially; that is, the failure of a single component precludes operation of that heliostat until a repair are completed. The user can specify heliostats that consist of any number of components depending on how much is known about the various possible heliostat failure mechanisms.

\section{Failure and Repair Distributions}

We assume all heliostats contain $N$ components $(N \geq 1)$, each of which represents a different failure mechanism. The cumulative distribution function for a Weibull random variable, shown in Equation 3.12, is used to describe time-to-failure for each component:

$$
F(x)=\frac{\beta}{\eta}\left(\frac{x}{\eta}\right)^{\beta-1} e^{-\left(\frac{x}{\eta}\right)^{\beta}} .
$$

Equation 3.13 represents the conditional cumulative probability distribution of $\tau$ given that the component's current age is $t$ and follows from the Weibull probability density function in Eqn. 3.12:

$$
F(\tau)=1-\exp \left[-\left(\frac{t+\tau}{\eta}\right)^{\beta}+\left(\frac{t}{\eta}\right)^{\beta}\right]
$$

We generate a time-to-failure for each component, for each heliostat by generating a uniform $[0,1]$ random variate via the WELL512 method (Panneton, L'Ecuyer, and Matsumoto) and taking the inverse of Equation 3.13 to obtain the lifetime, $\tau$. For a given component, this sampling occurs once at the beginning of the simulation and again upon completion of each repair. The component's operational age, $t$, is used as input if the repair restores a component to a "good-as-old" state; otherwise, the age may be reset to zero if a repair event restores the component to a "goodas-new" state. An analogous process is used to generate repair times from exponential random variables, whose cumulative distribution functions are equivalent to equation 3.13 with $\beta=1$; we assume the time required to restore a component does not depend on age.

\section{Repair Policies}

Upon failure of one (or more) of a heliostat's components, it is placed in a repair queue according to a prioritization scheme. Because there exists a potentially wide range of mean component repair times, and heliostat productivity varies by location in the field, a variety of nuanced approaches are possible in determining the queuing/repair process. We investigated five different strategies:

1. no prioritization, i.e., repairs assigned in random order (baseline strategy);

2. repairs prioritized by highest-to-lowest annual average heliostat performance of the failed heliostat;

3. repairs prioritized by shortest-to-longest expected value (mean) repair time of the failed component(s); and,

4. repairs prioritized by largest-to-smallest ratio of annual average heliostat performance of the failed heliostat to mean repair time of the failed component(s).

Option 2 delays repair of less-efficient outer heliostats in favor of more-efficient inner heliostats, as down-time of the less-efficient heliostats imposes a smaller penalty on overall performance-weighted availability. Option 3 delays long-duration repairs in favor of quick repairs in expectation, but requires knowledge of expected component repair time, possibly based on historical maintenance data. Option 4 uses the ratio of the measure for option 2 to that for option 3 to determine repair priority. Overall availability at any point in time is computed from a performanceweighted average given the set of available heliostats; that is, the availability is equal to the sum of the annual energy output of each available heliostat, divided by that of all heliostats in the field.

\section{Inputs and Outputs}


Table 5. Input parameters used by the heliostat availability model. Parameter and variable names match those used in DAO-tk scripting interface

\begin{tabular}{|c|c|c|c|}
\hline Name & Data type & Description (units) & Default value \\
\hline plant_lifetime* & Integer & Simulated plant life (years) & 30 \\
\hline avail_model_timestep & Floating-point number & Simulation step length (hours) & 24 \\
\hline om_staff** & Integer & $\begin{array}{l}\text { number of O\&M staff hired in each } \\
\text { year }\end{array}$ & NA \\
\hline om_staff_max_hours_week & Floating point number & $\begin{array}{l}\text { Maximum labor allowed per staff } \\
\text { member (hours/week) }\end{array}$ & 35 \\
\hline n_helio_sim & Integer & Number of & 1,000 \\
\hline solar_resource_file* & String & File path to TMY weather file & “" \\
\hline helio_repair_priority & String & Prioritization policy & "PERF_OVER_MRT" \\
\hline helio_comp_mtr & $\begin{array}{l}\text { Vector of floating-point } \\
\text { numbers }\end{array}$ & $\begin{array}{l}\text { Mean time to repair for each } \\
\text { component (hours) }\end{array}$ & \{\} \\
\hline helio_comp_weibull_shape & $\begin{array}{l}\text { Vector of floating-point } \\
\text { numbers }\end{array}$ & $\begin{array}{l}\text { Shape parameter } \beta \text { for each } \\
\text { component (unitless) }\end{array}$ & \{\} \\
\hline helio_comp_weibull_scale & $\begin{array}{l}\text { Vector of floating-point } \\
\text { numbers }\end{array}$ & $\begin{array}{l}\text { Scale parameter } \eta \text { for each compo- } \\
\text { nent (unitless) }\end{array}$ & \{\} \\
\hline helio_comp_repair_cost & $\begin{array}{l}\text { Vector of floating-point } \\
\text { numbers }\end{array}$ & Repair cost for each component (\$) & \{\} \\
\hline avail_seed & Integer & $\begin{array}{l}\text { Random number generator seed } \\
\text { (unitless) }\end{array}$ & 123 \\
\hline
\end{tabular}

Table 5 details the following user-specified inputs to the heliostat availability model, which are accessible via the DAO-tk scripting interface. Inputs to our model include: (i) the assumed plant lifetime; (ii) the simulation step length; (iii) the number and weekly labor limits of O\&M staff members; (iv) the number of heliostats to simulate, which may be smaller than the number of heliostats in the design to decrease computation time; (v) the repair prioritization policy, for which the default input corresponds to option 4; (vi) repair costs by component, which we assume is constant for each component and excludes labor costs; (vii) the parameters of each distribution governing a component's lifetimes or repair times; and, (viii) a seed for random number generation.

Table 6 summarizes the outputs provided by the heliostat availability model after the simulation is complete. These outputs include both the mean and a time series of field availability, and summary statistics for the annual number and cost of repairs.

Test Case

Table 6. Output parameters generated by the heliostat availability model. Parameter names match those used in DAO-tk scripting interface

$\begin{array}{lll}\text { Name } & \text { Data type } & \text { Description (units) } \\ \text { n_repairs* } & \text { Integer } & \text { Number of repairs over time horizon (components) } \\ \text { staff_utilization } & \text { Floating-point number } & \text { O\&M staff utilization (fraction) } \\ \text { heliostat_repair_cost_y1** } & \text { Floating-point number } & \text { Heliostat repair cost in year 1 }(\$) \\ \text { heliostat_repair_cost } & \text { Floating point number } & \text { Total heliostat repair cost }(\$) \\ \text { avg_avail } & \text { Floating-point number } & \text { Average field availability over time horizon (fraction) } \\ \text { avail_schedule* } & \text { Vector of floating-point } & \text { Time-series of field availability at each timestep (fraction) } \\ & \text { numbers } & \\ \text { n_repairs_per_component } & \text { Vector of floating-point } & \text { Average annual heliostat component repairs (components) } \\ & \text { numbers } & \end{array}$


For the purposes of demonstrating the features of the model, we selected a set of six components per heliostat. In the absence of data to support empirically-driven parameters, our test's failure and repair distributions are made up to stipulate (i) a high constant rate of control failures that can typically be repaired quickly, (ii) moderately frequent mirror, elevation drive, and azimuth drive failures which increase over time and require a moderate amount of time to repair, and (iii) infrequent support structure failures which require an exceedingly long repair time. Differences between specified component mean repair times are exaggerated to demonstrate model functionality and to illustrate differences in repair prioritization schemes, and range from 2 hours (e.g., controls) to >50 hours (e.g., support structure) in the results that follow.

Table 7 displays the collection of components that we use for initial testing of the model, as well as the parameters of the distributions that govern the lifetimes and repair times for each component. These parameters have no origin in actual heliostat operational data and are intended solely for demonstration of the model functionality. Some parameters are based very loosely on publicly available online databases of Weibull failure parameters for common mechanical components (Barringer), but we do not necessarily expect this set of parameters to realistically describe a heliostat field.

Table 7. Component parameters for model demonstration

$\begin{array}{llllll}\text { Component } & \beta & \eta(\mathrm{hr}) & \begin{array}{l}\text { Median time to } \\ \text { failure }(\mathrm{yr})\end{array} & \begin{array}{l}\text { Mean time to } \\ \text { repair (hr) }\end{array} & \begin{array}{l}\text { Repair to good- } \\ \text { as-new }\end{array} \\ \text { Control } & 1.0 & 12,000 & 0.95 & 2.0 & \text { True } \\ \text { Elevation drive } & 2.0 & 75,000 & 7.1 & 8.0 & \text { True } \\ \text { Azimuth drive } & 2.0 & 75,000 & 7.1 & 8.0 & \text { True } \\ \text { Mirror } & 1.5 & 100,000 & 8.9 & 20.0 & \text { True } \\ \text { Mirror support } & 1.5 & 300,000 & 26.8 & 50.0 & \text { True } \\ \text { Pedestal } & 1.2 & 1,000,000 & 84.1 & 100.0 & \text { True }\end{array}$

Figure 9 illustrates an example of simulated hourly availability over a 25-year simulation period for a field of 8,660 heliostats, each of which contains the six components described above. Simulations were conducted with 10 repair staff, and the color of each line in Figure 9 corresponds to the four different repair prioritization options described above. Figure 9 uses hourly time resolution and each simulation required approximately 1-2 minutes of simulation time for the 30-year simulation period. However, use of time steps as long as 24 hours adequately captured field availability within the range of values achieved from different realizations of the stochastic model.

Figure 9 shows that for this collection of probability distributions that govern component lifetimes and repair times, options 1 and 2 (repairs prioritized by random order or heliostat performance) each produced significantly lower availability during the later years than options 3 and 4 (repairs prioritized by lowest-to-highest mean repair time, with or without prioritizing on heliostat productivity); however, option 2 (i.e., prioritized by productivity) provided a slight improvement in availability when computed as an efficiency-weighted average. Hourly availability is nearly flat during the first few years of simulation, followed by a sharp decrease in availability after approximately years 5-10. This is due to the increased failure rate for components which wear out over time (i.e., $\beta>1$ ). In this example, we observe the interesting result that simply prioritizing repairs by repair time among the available staff significantly increased mean field availability, while there was limited marginal benefit in also prioritizing repairs according to performance. This effect can be at least partially attributed to the wide range of component mean repair times, when compared to the range of heliostat performance. All test cases to date indicate a higher average field productivity when prioritizing short-duration repairs; however, the relative difference between repair-prioritization schemes decreases with the variability in mean repair time between components.

\subsubsection{Soiling and degradation}

The efficiency of CSP plants depends on the reflectance of the solar field's mirrors, which are negatively impacted by the accumulation of dust and particulate matter over time, or soiling, and permanent damage to the reflective surface, or degradation. The purpose of the soiling and degradation models is to approximate activity in the optical field that reduces the reflectivity of the mirrors due to these two environmental factors. The stochastic model steps 


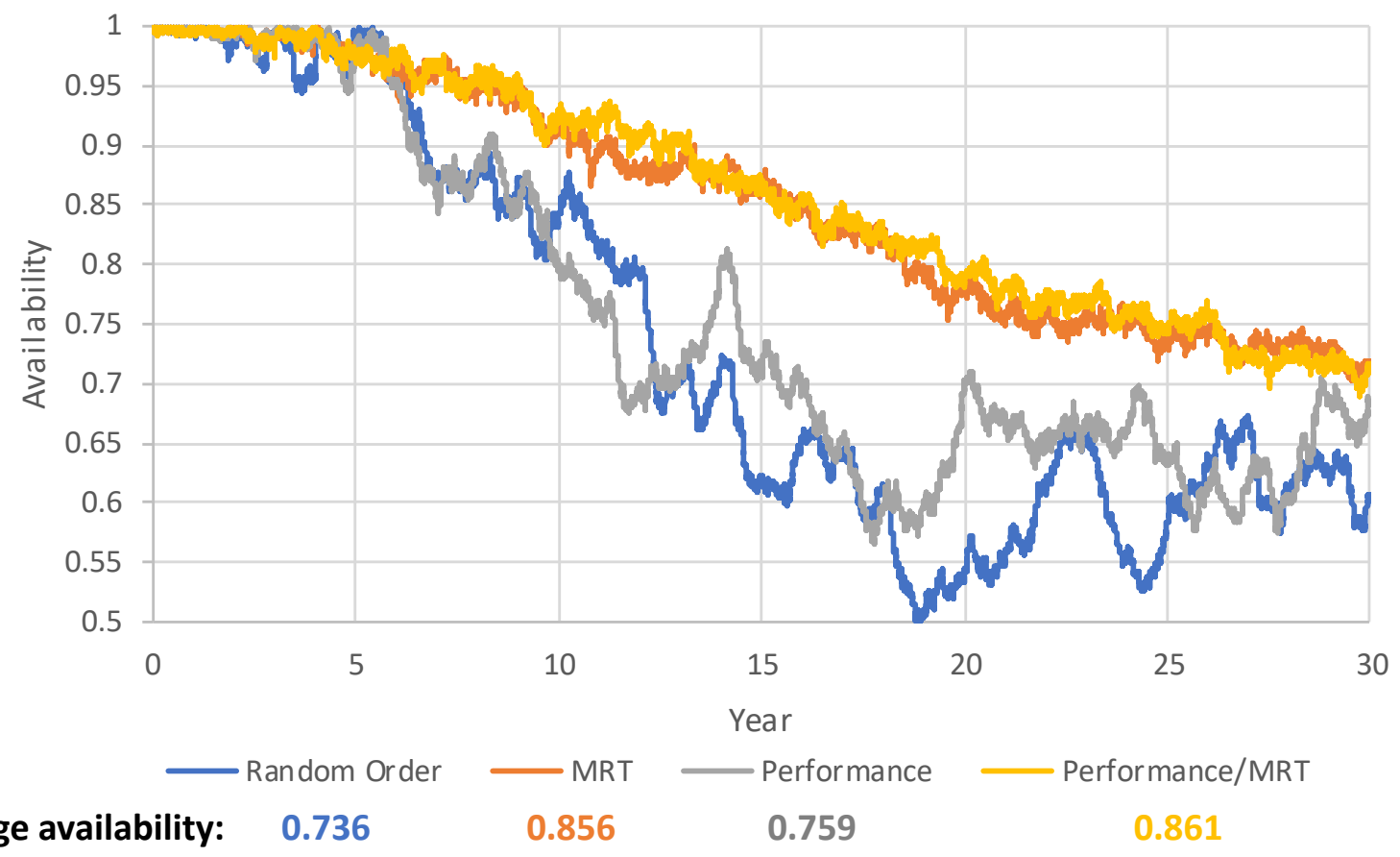

\section{$\begin{array}{lllll}\text { Average availability: } & 0.736 & 0.856 & 0.759 & 0.861\end{array}$}

Figure 9. Hourly solar field availability for a case study using 10 O\&M staff; each line denotes a different repair prioritization policy. Component lifetime and repair time distributions are described in Table 7

through time, obtains realizations of soiling and degradation rates at specified intervals, and applies these rates to the heliostats on an hourly basis. Each wash crew is assigned a subset of the heliostats in the solar field, and in the simulation, rotates through their assigned heliostats, cleaning the mirrors and replacing them as needed. The outputs of this model include the operations and maintenance costs associated with the mirrors in the solar field, the number of wash vehicles to procure, and an assignment of heliostats for each wash crew.

Sarver, Al-Qaraghuli, and Kazmerski provide a review of the literature dedicated to measuring the effects of soiling on the performance of CSP and photovoltaic plants, and indicate that while efficiency reductions over time vary widely by location, field tests showed that arid and windy locations are subject to efficiency losses as high as $10 \% /$ day for a horizontal surface. Several models are proposed for estimating the specular reflectivity of a single heliostat (Pettit; Gee et al.; Meyen et al.), and Zhu et al. develop a method to characterize a the efficiency of a CSP plant's collection system using measurements from a subset of the heliostats in the solar field (Zhu, Kearney, and Mehos).

Several technologies exist to mitigate the impact of soiling on solar field efficiency, including anti-soiling coatings (Hunter et al.; Sarver, Al-Qaraghuli, and Kazmerski) and automated, self-cleaning technologies (Mazumder et al.; Mazumder et al.; Joglekar et al.). Currently, the most cost-effective method for removing dust to minimize soiling losses is the use of wash crews, who manually clean the mirrors with a brush and a solution of demineralized water and a mild detergent (Fernández-García et al.). Some vehicle technologies optimize the routing of wash crews to maximize the rate at which mirrors are washed in a power tower facility's solar field (Alon et al.).

The literature dedicated to wash crew scheduling is largely restricted to policies that determine either an optimal frequency of cleaning all mirrors in the solar field, or a threshold policy that dictates a cleaning of the field when reflectance falls below some threshold. Optimal, site-specific cleaning frequencies exist to balance the costs of cleaning with efficiency degradation due to dust (Bergeron and Freese). Several works track soiling-related losses over time for implementations of CSP parabolic troughs, and provide recommended cleaning frequencies according 
to each location's observations (Deffenbaugh, Green, and Svedeman; Mani and Pillai). Wolferstetter et al. develop a model that seeks an optimal wash crew policy for parabolic trough plants, in which the candidate policies offer either a constant cleaning rate or cleaning with all available crews when the field availability falls below a certain threshold (Wolfertstetter et al.); the authors propose another policy in which additional crews are assigned when a second threshold is passed. Similar condition-based cleaning policy and determine the optimal time-varying threshold cleaning policy using a Markov decision process in (Truong Ba et al.). While these models area appropriate for CSP technologies in which each heliostat provides a similar amount of energy to the plant over time, they do not consider the unequal productivity of heliostats in a power tower configuration.

Field data for the degradation of mirrors is fairly limited, and performance by test varies widely. A literature review of both accelerated ad outdoor exposure tests for glass-backed silver mirrors, silvered aluminum reflectors, and silvered polymer reflectors in (García-Segura et al.) shows that specular reflectivity degradation rates vary from $0.75 \%$ per year (Almanza et al.) to more than 50\% in a single year; however, outdoor exposure tests in recent years have shown that specular and hemispherical reflectivity degradation rates have improved significantly when compared to older technologies.

The improved soiling and degradation models that we develop feature two significant improvements. The first upgrade determines the number of vehicles and wash crews to maintain the solar field that minimizes the sum of revenue losses to soiling and capital and labor costs, by solving a dynamic program that allows for washing policies that allow for an unequal number of heliostats to be assigned to different wash crews. This development (i) allows for policies in which wash crews prioritize the most productive heliostats nearest the receiver by washing them more frequently, and (ii) removes an integer variable from the outer loop optimization, which should improve run times for the broader toolkit. The second improvement calculates an optimal threshold for replacement of the mirrors under an assumed annual energy output and constant mirror degradation rate.

\subsubsection{Wash crew hiring subproblem}

We describe the wash crew hiring subproblem below, as a mixed-integer nonlinear program.

Table 8. Components of the wash crew subproblem formulation

\begin{tabular}{|c|c|c|}
\hline \multicolumn{3}{|c|}{ Sets and Indices } \\
\hline$i$ & & heliostat collections (e.g., rings), $\in \mathcal{I}=\{1, \ldots, l\}$ \\
\hline$j$ & & candidate wash crews, $j \in \mathcal{J}=\{1, \ldots, J\}$ \\
\hline \multicolumn{3}{|r|}{ 管 } \\
\hline$d_{i}$ & MWh & annual DNI collected by ring $i$, with no soiling \\
\hline$t_{i}$ & $\mathrm{~h} /$ group & time to wash heliostat collection $i$ \\
\hline$c_{j}$ & $\$$ & annual cost of hiring crew $j$ \\
\hline$p$ & $\$ / M W h$ & revenue per unit DNI sent to receiver, after conversion to electricity \\
\hline \multicolumn{3}{|c|}{ Functions } \\
\hline$f(\cdot)$ & & average soiling-related efficiency loss of a heliostat, according to wash frequency \\
\hline \multicolumn{3}{|c|}{ Decision variables } \\
\hline$X_{i j}$ & & proportion of heliostat collection $i$ assigned to wash crew $j$ \\
\hline$Y_{j}$ & $\mathrm{~h}$ & time for crew $j$ to wash all assigned mirrors \\
\hline$Z_{j}$ & & one if crew $j$ is hired, and zero otherwise \\
\hline
\end{tabular}

Formulation 


$$
\begin{aligned}
\min _{X, Y, Z} Z= & p \cdot \sum_{i \in \mathcal{I}}\left(d_{i} \sum_{j \in \mathcal{J}} X_{i j} \cdot f\left(Y_{j}\right)\right)+ \\
& \sum_{j \in \mathcal{J}} X_{i j}=1, \forall i \in \mathcal{I} \\
& Y_{j}=\sum_{i \in \mathcal{I}} t_{i} \cdot X_{i j}, \forall j \in \mathcal{J} \\
& 0 \leq X_{i j} \leq Z_{j}, \forall i \in \mathcal{I}, j \in \mathcal{J} \\
& Y_{j} \geq 0, \forall j \in \mathcal{J} \\
& Z_{j} \in\{0,1\}, \forall j \in \mathcal{J} .
\end{aligned}
$$

\section{Discussion}

We seek, via the objective function (3.14a), hiring and assignment decisions for wash crews that provide the minimum lost revenue for the plant, plus the annualized cost of hiring wash crews and purchasing equipment. The revenue parameter $r$ assumes that the revenue gained by absorbing DNI is linear in the DNI collected at the central receiver; while dispatch optimization is required to avoid the curtailment of collection due to a lack of available thermal energy storage (Wagner et al.; Wolfertstetter et al.), we adopt this simplification for our planning model. We have developed results showing that the objective in (3.14a) is convex when the average heliostat efficiency function is convex and nondecreasing, and the binary variables, $Z_{j}, j \in \mathcal{J}$, are fixed. Constraint (3.14b) enforces the assignment of each collection of heliostats to one or more mash crews. Constraint (3.14c) tracks the time it takes for a wash crew to perform a cleaning of all assigned heliostats. Constraints (3.14d)-(3.14f) provide nonnegativity, binary, and simple-bound restrictions.

While the formulation above is nonconvex in general, we can recast this problem as a dynamic program for our application to obtain a solution that includes (i) the number of wash crews to hire, and (ii) an assignment of wash crews to heliostats in the field. This requires an equal amount of time to clean each heliostat, which may be reasonable if the allocation of heliostats to wash crews yields connected regions, and if wash vehicle travel time between stops is negligible compared to time spent cleaning the mirrors.

\section{Heliostat replacement policy}

For our degradation model, we have developed a heliostat replacement policy in which the mirrors are replaced at varying thresholds according to the estimated annual power output of each heliostat. Much like the soiling model described above, we consider a monotonically increasing function to represent a mirror's expected degradation over time, and we assume that each mirror in the solar field is subject to the same degradation function according to that mirror's age. Let $h(t)$ be the expected degradation-related efficiency loss when after a time interval of length $t$, and let $r$ be the (fixed) replacement cost for a mirror. Then, the expected loss rate over the replacement interval for heliostat $i$, which we term $L_{i}(t)$, is

$$
L_{i}(t)=p \cdot d_{i} \cdot g(t)+\frac{r}{t}, \forall i \in \mathcal{I}
$$

if $L_{i}(t)$ is convex, then this loss rate is minimized by solving for $L^{\prime}(t)=0$, which, for a linear degradation function, i.e., $g(t)=a t / 2$, with $a \in \Re_{+}$fixed, yields the following optimal solution $t^{*}$ :

$$
t^{*}=\sqrt{\frac{2 r}{p \cdot d_{i} \cdot a}} .
$$

If the model assumes a degradation rate that accelerates over time, i.e.,

$$
g(t)=b t \cdot c^{t}
$$


then the loss rate for heliostat $i, L_{i}(t)$ is as follows:

$$
\begin{aligned}
L_{i}(t) & =p \cdot d_{i} \frac{\int_{0}^{t} x \cdot(b x) c^{x} d x}{t}+\frac{r}{t} \\
& =p \cdot d_{i} \cdot \int_{0}^{t} \cdot\left(b x^{2}\right) c^{x} d x+\frac{r}{t} \\
& =p \cdot d_{i} \cdot\left(\frac{b t}{\log (c)}-\frac{b}{\log ^{2}(c)}\right) c^{t}-\frac{1}{t \cdot \log ^{2}(c)}+\frac{r}{t} \\
& =p \cdot d_{i} \cdot\left(\frac{b t}{\log (c)}-\frac{b}{\log ^{2}(c)}\right) c^{t}-\frac{1-r \log ^{2}(c)}{\log ^{2}(c) \cdot t}
\end{aligned}
$$

While an closed-form solution to equation (3.18) does not exist, $L_{i}(t)$ is convex when $g(t)$ is defined by equation (3.17) with $b \geq 0$ and $c \geq 1$, so we can obtain the replacement threshold that minimizes $L_{i}(t)$ solution using bisection.

\section{Implementation notes and run times}

The soiling and degradation models are implemented in C++ as a library in NREL's DAO-tk project on GitHub. Run times for the wash crew hiring problem and calculation of optimal replacement thresholds took less than two seconds on a personal laptop across a collection of case studies.

\section{Case studies}

For the case studies that follow, we adopt a design obtained using SolarPILOT (Wagner and Wendelin) that includes 8,662 heliostats; the data from SolarPILOT include each heliostat's location and its estimated annual energy collection. Table 9 details the assumed inputs to our problem for the sensitivity analysis; we assume that lost revenue is subject to a discount rate, while the labor and materials costs for wash crews remain constant for the plant's lifetime. The simulations assume independent gamma processed govern the soiling and degradation of each mirror in the field over time, similar to the process used in (Yuan, Pandey, and Bickel).

\subsection{Sensitivity analysis}

Sensitivity analysis allows us to understand the impact several assumptions have on the recommended number of wash vehicles to procure and crews to hire. Figure 10 displays the optimal number of wash crews for the baseline case described in Table 9, but also varies key parameters by up to 50\%. The results show that while wash crew vehicle cost does not have a significant impact on the decision, revenue and soiling rate increases lead to an increase in the number of wash crews to hire, while a reduction in wash crew cleaning rate or hourly costs leads to the hiring of additional wash crews.

\subsection{Modeling improvements}

To show the relative value of the wash scheduling and mirror replacement policies, we show the estimated sum of revenue loss and replacement costs as a function of the different policies we adopt. In the first case, we assume that wash crews have an equal allocation of mirrors in the optical field, no mirrors are replaced due to degradation, and the number of wash crews hired approximately yields the soiling-related reflectivity of $95 \%$ from the baseline case in Wolfertstetter et al. In the second case, we optimize the number of wash crews. In the third case, we allow all mirrors to be replaced with the same reflectivity threshold, which we determine by using the average annual output per mirror in the solar field. In the fourth case, we calculate an optimal replacement threshold for each heliostat. Finally, in the fifth case, we allow for the wash crews to maintain an unequal number of heliostats, in an effort to prioritize the higher performing ones nearest to the collector. Table 10 shows the summary statistics for the performance measures we select, which are average field reflectivity (net of soiling and degradation losses), number of replacements, and change in relative losses to soiling, degradation, and replacement costs. We build a confidence interal (CI) using 100 replicates of each optical field simulation common random number substreams were used for each case. 
Table 9. Inputs to case studies for soiling and degradation models

\begin{tabular}{|c|c|c|c|c|}
\hline Parameter & Units & Base Value & Source & Notes \\
\hline Crew wash rate & $\frac{m^{2}}{h}$ & 1,840 & {$[1]$} & $\begin{array}{l}50 \% \text { reduction from trough } \\
\text { wash rate }\end{array}$ \\
\hline Vehicle capital cost & $\$$ & 100,000 & [2], p. 139 & \\
\hline Wash crew cost rate & $\frac{\$}{\text { crew-hr }}$ & 50 & [2], p. 138 & $\begin{array}{l}\text { Mean for Mr. Twister; } \\
\text { includes labor, fuel, water, } \\
\text { vehicle maint. }\end{array}$ \\
\hline Vehicle lifetime & $\mathrm{yr}$ & 10 & [2], p. 139 & \\
\hline Plant lifetime & $\mathrm{yr}$ & 35 & & \\
\hline $\begin{array}{l}\text { System efficiency / field } \\
\text { efficiency }\end{array}$ & fraction & 0.331 & [3], Table 1 & \\
\hline Dumping, heat loss & fraction & 0.101 & [3], Table 1 & \\
\hline Average revenue & $\frac{\$}{\mathrm{kWh}}$ & 0.137 & [4], Table 3 & Crescent Dunes \\
\hline Soiling rate/day & fraction & 0.0036 & [5] & $\begin{array}{l}\text { Midpoint of range for even } \\
\text { US sites }\end{array}$ \\
\hline Degradation/year & fraction & 0.0275 & [6] & \\
\hline Mirror cost & $\frac{\$}{m^{2}}$ & 23.06 & [2], Table 3-22 & \\
\hline Discount rate & $\%$ & 6 & [3] & \\
\hline $\begin{array}{l}\text { 1. Wolfertstetter et al. } \\
\text { 2. Kolb et al. } \\
\text { 3. Röger et al. } \\
\text { 4. Mehos et al. } \\
\text { 5. Deffenbaugh, Greer } \\
\text { 6. Nostell, Roos, and }\end{array}$ & deman & & & \\
\hline
\end{tabular}

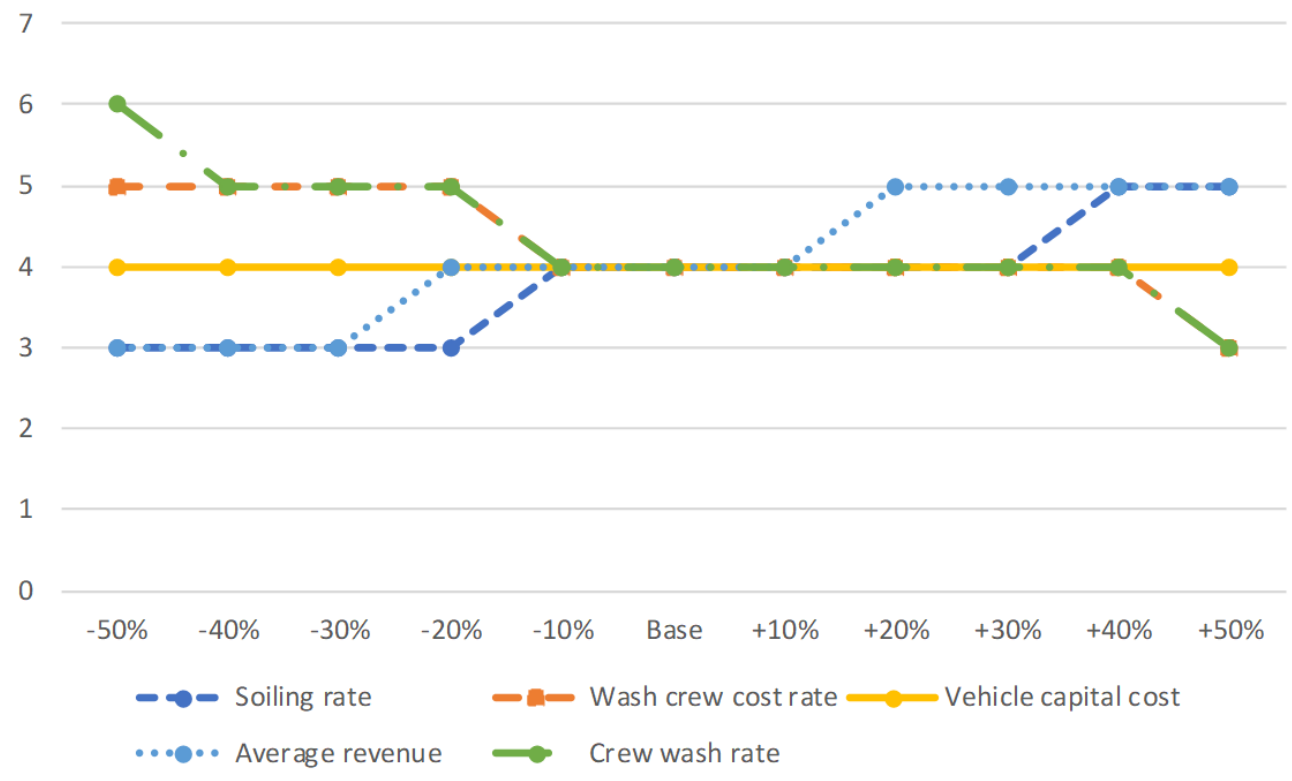

Figure 10. Sensitivity plot showing the optimal number of wash crews as a function of changing parameter values; base values are available in Table 9 
Table 10 proves that beyond the replacement of mirrors that break due to weather or maintenance, it is worthwhile to measure specular reflectivity to assess whether or not a proactive replacement is warranted. While optimizing the replacement threshold is useful for the mirrors in the solar field, finding a separate replacement threshold for each mirror according to its annual energy output doesn't provide a material difference in profit. Finally, while hiring the correct number of wash crews is critical to maximizing profits, there is limited upside to using a heliostat washing policy in which crews maintain the mirrors at unequal frequencies according to power output in this case study.

\subsubsection{Power cycle availability}

There is an increasing need for CSP systems to frequently startup, shutdown, and ramp up electricity generation to both maximize profit (by meeting peak pricing times) and to compensate for the rising share of renewable energy sources that do not provide constant power throughout the day. This cycling and fast ramping causes increased wear and tear on the components of the power plant when compared to baseload, i.e., steady state, operations (Keatley). Though previous research has focused on optimizing the dispatch of electricity to maximize profits (Wagner et al.), there is a need to better quantify how different dispatch policies impact the frequency of partial or full plant shutdowns caused by failures in the power cycle. Additionally, there is a desire to explore the impact of these events on total revenues, as the presence of thermal storage and intermittent nature of solar irradiance may lead to maintenance-related losses that vary from those of a plant configured for baseload operations. Finally, we are interested in evaluating the impact that cycling and ramping costs have on optimized dispatch decisions over short timeframes, and determining the penalties that yield maximum revenues over the operating horizon while accounting for component failures in the power cycle.

We describe a maintenance and failure simulation that is integrated with an optimization dispatch model in order to account for the impacts of excessive cycling and ramping. We use a Monte Carlo-based procedure to simulate stochastic events in a power plant, when provided plant specifications and hourly dispatch decisions from an optimization model. These events then serve as inputs to the next iteration of the optimization model that is successively solved on a rolling horizon. This method is used to obtain dispatch policies while accounting for component reliability and maintenance requirements. In particular, we are interested in simulating large component failures and maintenance events that may be pre-specified (e.g., a maintenance event occurs for every five thousand hours of operation) or stochastic in nature (e.g., component failure inter-arrival times for the heat exchanger are exponentially distributed with a mean time of 10,000 hours of use). The model is used to validate certain inputs to the optimization model, such as the expected cost of a cold start, based on optimized dispatch (e.g., Kumar et al.). Our research concerns simulating maintenance and failure events for critical components of the power cycle of the CSP plant, described in the figure as the Steam Generation System and Power Block. Section 3.3.5.2.1 describes the specific components modeled in the simulation.

Since there is usually not enough storage to operate continuously at full capacity and there may be times during the day when it is not profitable to sell electricity, it desirable to start and stop (cycle) the plant often and quickly ramp up electricity generation to capacity in order to meet peak pricing periods. Additionally, the varying natures of solar radiance, weather, and electricity prices induce cycling operations and fast ramping (Wagner et al.). Though a CSP system has a greater ability to vary power output throughout the day over traditional power plants that are designed to operate at a fixed capacity, cycling and fast ramping cause additional wear and tear on the critical components of a CSP plant (Keatley).

The goal of the research presented in this section is to improve the dispatch planning of a power plant by adding impacts of maintenance, failure, and repair events to understand the tension between the advantages of meeting dynamic pricing schedules and the costs of additional wear and tear on plant components due to cycling and fast ramping. We develop a novel maintenance and failure simulation that uses the operational decisions of the plant to change occurrences of component failures. We integrate the simulation with an existing dispatch mixed integer program (MIP) using a rolling solution method in order to provide a realistic dispatch schedule that incorporates component availability in real-time. 


\subsubsection{Literature review}

Many factors influence the failure rates of components in a power plant. Thermo-mechanical fatigue, creep, corrosion, load cycling, and fast ramping are some conditions which negatively impact these failure rates (Benato, Stoppato, and Bracco, Tică et al.). A variety of researchers examine the impact of cycling and fast ramping on the wear and tear of traditional power plants as they adapt to meet the variable demand due to increases in renewable energy sources on the grid and deregulated energy markets (e.g., Mirandola, Stoppato, and Lo Casto; Stoppato et al.; Keatley, Shibli, and Hewitt; Guédez, Spelling, and Laumert; Aminov and Kozhevnikov). Kumar et al. use industry data to quantify the cost of cycling the power plant, both financial and in increased expected forced outage rates. A CSP plant must also vary power generation due to TES limitations, weather conditions, and to maximize profit by optimizing a dispatch schedule around pricing profiles (Wagner et al.). This introduces decisions regarding how often to start and stop the CSP plant power cycle or how quickly to ramp up generation to meet peak pricing profiles, while minimizing maintenance costs and down time due to failures.

Dispatch optimization of a power plant, that incorporates operations and maintenance costs, is a studied research area. As described in Section 2, Wagner et al. optimize the dispatch of a CSP plant with thermal storage using a MIP. They consider the impacts of operating decisions on maintenance by including penalties for startups and ramping.Santos and Uturbey develop a mixed integer nonlinear program to optimize dispatch in a combined heat and power plant. They model maintenance costs by applying a constant penalty to each startup and shutdown. Liu et al. determine profit ranges of a virtual power plant using a deterministic dispatch model with interval optimization, whereby they determine outcomes using the high, low, and middle interval of uncertain parameters. They model maintenance costs as a linear function of subsystem outputs. Our research uses a similar deterministic dispatch model to that of Wagner et al., but rather than using a constant penalty for a subset of dispatch decisions, we let the dispatch decisions serve as input to a Monte Carlo failure simulation, which, in turn, generates component failures that influence revenues and costs. Wu, Shahidehpour, and Khodayar incorporate ramping penalties for thermal generators in a unit commitment model; however, generator availability in this setting is generally addressed by incorporating spinning reserve (e.g., Aminifar, Fotuhi-Firuzabad, and Shahidehpour) and/or n-1 reliability constraints (e.g., O'Neill et al.; Hedman et al.), whereas we directly model forced outages for a single plant.

The majority of models in the literature separate the power plant into a collection of system components and simulate the operating states (such as full operational, partially operational, etc.) in order to conduct analysis or optimization. Tică et al. develop an adaptation of a simulation to determine a minimum-time startup sequence for a combinedcycle power plant. The goal of their work is similar to ours in that we also develop a simulation that is integrated with an optimization model; however, different stresses define the condition of each component, and spontaneous component failures are not considered. Multiple works use a Markov chain or state space method to calculate steady state failure rates (e.g., Zare; Kumar, Sharma, and Tewari). Markov systems are used when the system can be characterized as possessing a "memory-less" process for failures (Haghifam and Manbachi). Frangopoulos and Dimopoulos use the state space method to provide a set of probabilities of the system being in a certain state at any point in time, and identify the most often occurring failure states, calculate availability and reliability of the system, and produce better estimates of system cost, profit, and output. They account for both active and passive redundancy using these methods. Godoy, Benz, and Scenna use a state space method to consider the impact of maintenance strategies on a natural gas combined cycle power plant. While these models are useful to characterize system reliability, they do not consider the impact of component failures on optimized dispatch decisions.

Most research identifying failure and repair rates is related to organic Rankine cycles and combined-cycle gas turbine power plants (e.g., Zare; Sabouhi et al.). Similar to our research, others investigate the trade-off between plant operations that maximize profit using peak pricing targets and the negative impact this strategy can have on the frequency of critical component failures. Mirandola, Stoppato, and Lo Casto modify components' equivalent lifetimes due to cycling or fast ramping by conducting analysis of a design and thermodynamic simulation under different dispatch strategies. Their research differs from ours in that it is focused on superheater pipes in a coal power plant and they use existing simulators to model component structural reliability. Aminov and Kozhevnikov evaluate fuel and turbine overhaul costs of a gas turbine power station under varying dispatch decisions. They use a procedure, similar to ours, whereby the equivalent lifetime of a component depends on its number of starts. While there is significant overlap 
between previous research and our application, there are critical differences in operation. Dispatch decisions in a CSP plant must consider varying solar and weather conditions, which change the amount of dispatchable thermal storage and increase the need for cycling and fast ramping. Additionally, much of the current research only considers modeling a few components instead of all critical subsystems in a power plant.

We simulate events using component failure mode distributions and repeat operating policies multiple times with Monte Carlo methods. The stochastic nature of component lifetimes, repair times, and failure probabilities leads to the use of Monte Carlo simulation, a process of repeating an experiment many times with randomly generated values for unknown parameters and using the distribution of results to make claims about the system (Fishman). Borgonovo, Marseguerra, and Zio develop a Monte Carlo simulation of components in an industrial plant to evaluate the effects that different maintenance and part renewal strategies have on component availability, costs, aging, and obsolescence. Marseguerra and Zio use a similar process to model component failures that are integrated with a genetic algorithm to optimize plant revenue, including profits and maintenance costs.

Several papers discuss CSP system modeling and remark on their performance and efficiency, but lack in-depth discussion of reliability (e.g., Menéndez et al.; Altés Buch; Powell and Edgar); similar work has been done on combined-cycle plants (). Other papers discuss reliability, but are concerned with optimizing plant design and operational scenarios, such as Aguilar et al. Similarly, Guédez, Spelling, and Laumert analyze turbine lifetimes and cost expenditures of CSP systems with varying amounts of TES. To the authors' knowledge, to date, there is not any research evaluating the impact that operational decisions have on component reliability when determining an hourly optimal dispatch schedule. Our research combines a model that simulates failures with a dispatch MIP that optimizes decisions to maximize profit. We perform a Monte Carlo simulation with these integrated models in order to develop more accurate power generation policies that consider component wear and tear, and to characterize the effects of cycling and ramping on both revenues and unplanned maintenance events.

Simulation optimization approaches are commonly used for cases in which the true objective function of a collection of decision variables is unknown, but may be estimated using simulation (April et al.; Fu, Glover, and April; $\mathrm{Chen,} \mathrm{Fu,} \mathrm{and} \mathrm{Shi).} \mathrm{In} \mathrm{a} \mathrm{sample} \mathrm{path} \mathrm{optimization} \mathrm{approach,} \mathrm{Monte} \mathrm{Carlo} \mathrm{simulation} \mathrm{techniques} \mathrm{are} \mathrm{used} \mathrm{to} \mathrm{gener-}$ ate multiple sample paths for uncertain parameters, which then serve as input to an optimization model that obtains optimal decisions in a multi-stage setting (e.g., Higle and Sen; Mak, Morton, and Wood). Other simulation optimization approaches evaluate a stochastic black-box function when provided initial values for decision variables as input, and then a new decision vector is selected either by exhaustion of a pre-defined collection for ranking and selection (Kim and Nelson), or by a search, which may be (i) tailored toward building a response surface (Kleijnen), (ii) random (e.g., Kushner and Yin), (iii) gradient-based (Andradóttir), or (iv) according to a metaheuristic (Olafsson, $\mathrm{Li}$, and $\mathrm{Wu}$ ). The search terminates when either the search space or computational budget is exhausted, or there is a failure to improve after a prespecified number of evaluations. While the parametric analysis on cycling costs we implement falls under ranking and selection, and we do optimize over samples of failure events, our approach differs from both classes of methods above because optimal decision variables under initial conditions serve as input to the simulation, and if any failure events occur, then the optimization model is solved again starting from the time of failure; this cycle of optimization and simulation occurs for a time block in an iterative fashion until no new failure events occur.

Some simulation optimization works involve the use of a rolling-horizon solution approach, in which a simulation occurs within each time block. Jung et al. use simulation to generate demand realizations, and then solve mixedinteger programming subproblems in each time block to obtain safety stock decisions for a supply chain management problem. Chang et al. iteratively use a heuristic scheduling policy with simulation to generate delay realizations, then obtain new schedules via metaheuristic for a yard crane scheduling problem. Unlike these applications, in which decisions for each subproblem (i.e., time block) are optimized and evaluated exactly once, our approach requires re-optimizing and re-simulating part of a subproblem when a spontaneous failure occurs.

\subsubsection{Integrated models}

Concentrating solar power plants are particularly interesting for study because: (i) there is a desire to operate in such a way to maximize profit by generating electricity during peak pricing times; and (ii) there is a need to protect 
the components from excessive wear and tear. The dispatch optimization model - here referred to as $\mathcal{R}-$ serves as input to the maintenance and failure simulation. We detail how plant operations are used as input to simulate maintenance, failure, and repair events. Finally, we describe how the reliability simulation integrates with the SAM and the dispatch optimization using a rolling solution method.

\subsection{Simulation model $(\mathcal{S})$}

The CSP maintenance, failure, and repair simulation, hereupon referred to as $(\mathcal{S})$, includes critical components in the power cycle of the plant and simulates preventative maintenance and wear and tear. Operational decisions from $(\mathcal{R})$, which may include excessive cycling or fast ramping, serve as input to $(\mathcal{S})$. The results of $(\mathcal{S})$ include failures, repair times, and reduced capacity and efficiency for each time period in which one or more components of the power cycle is down due to failure.

We include in $(\mathcal{S})$ the critical components in the power cycle that have a well-defined, negative impact on the capacity and/or efficiency of the power cycle upon failure. We do not consider reliability for components outside of the Rankine cycle, such as the heliostats and receiver. We consider failure events for the following components of the Steam Generation System and Power Block in Figure 1: (i) turbines; (ii) feedwater heater, boiler, and hot salt pumps; (iii) feedwater heaters (i.e., steam-to-water heat exchangers); (iv) condenser fans; (v) condenser heat exchangers; (vi) reheaters; (vii) superheaters; (viii) boilers; and, (ix) economizers. Each component can have several parts and failure modes. Additional components of interest and associated failure modes can be added.

The failure of some components (e.g., generator) may preclude power cycle operations during repair; others (e.g., a single condenser bay) may impose a limit on power output by imposing a capacity and/or an efficiency degradation, but still allow the power cy cle to operate when they fail. A capacity degradation, resulting in a new relative capacity, occurs if the failure of a component reduces the maximum thermal energy consumption of the power cycle, thereby limiting the amount of electrical power that can be generated. A relative capacity parameter, $F_{t}^{c a p}$, is applied to the maximum thermal power consumption constraint in $(\mathcal{R})$, here shown in constraint (3.19). An efficiency degradation, resulting in a new relative efficiency, occurs if the failure of a component reduces the amount of electrical power that can be generated for a given amount of thermal power consumed. A relative efficiency parameter, $F_{t}^{e f f}$, is applied to the maximum electrical power generation constraint in $(\mathcal{R})$, shown in constraint (3.20). Definitions are provided in Table 11.

$$
\begin{aligned}
& x_{t} \leq F_{t}^{\text {cap }} Q^{u} y_{t} \forall t \in \mathcal{T} \\
& \dot{w}_{t} \leq F_{t}^{e f f} f\left(x_{t}, y_{t}, \eta\right) \quad \forall t \in \mathcal{T}
\end{aligned}
$$

Though the CSP production plant consists of relatively new technology in the collection system, many components in the power cycle have been used in other thermal generation plants for decades. Here, we use data from components in nuclear power plants as the source of the failure modes and distributions. A report from the Idaho National Laboratory (INL) (Eide et al.) provides failure distributions for nuclear energy production components that are analogous to those in the CSP components we model. The INL report uses four years of data from over a hundred nuclear power plants to detail failure modes for components. The air-cooled condenser differs from the cooling towers used for the systems described in the INL report, so for these components, we use reliability data from the standard developed by IEEE for commercial power systems.

The failure modes detailed in the INL report include probability distributions for different potential events for each component. The two types of failure modes can be differentiated as expenditure of lifetime, i.e., the number of operating hours that pass before a component ceases to function; and, a failure probability, i.e., an event that only occurs when the plant starts to generate electricity. Probabilities are governed by beta distributions, and component failure rates are governed by gamma distributions in the INL report; hence, lifetimes are governed by inverse-gamma distributions (Forbes et al.). Example failure modes with inverse-gamma distributions include external leaks and failure while operating. Some components, such as pumps, have a different failure rate for the first hour a component operates from the rate for when a component has been operating for more than one hour. 
Lifetimes of component failure modes are reduced when a component is operating using the component's hazard rate, or its rate of lifetime degradation during operations without the effects of fast ramping. The hazard rate dictates how fast a lifetime is reduced for each period of operation; e.g., a hazard rate of 1.1 would reduce a lifetime by one hour and six minutes for every hour of operation.

Random variates are generated using the WELL512 implementation developed by Panneton, L'Ecuyer, and Matsumoto. Specifically, we use WELL512 to obtain a uniform $[0,1]$ random variate, and then calculate component lifetimes and repair durations by evaluating the inverse of each event's cumulative distribution function at this value. Gamma- and beta-distributed random variates are generated in the simulation as needed for each failure mode, for each component. Algorithms detailed in Fishman, in combination with WELL512 random variates, are used to generate these lifetimes and probabilities in order to reduce the dependency on external libraries.

\section{Failure and repair events}

The INL report (Eide et al.) describes failure distributions that differ by component and by the plant's operating state, which we define as one of the following: (i) off-line; (ii) standby mode; (iii) the start of online mode (i.e., the power cycle starts to generate electricity); (iv) the first hour of online mode; and (v) ongoing online mode (i.e., the plant has been in online mode for more than one hour). No failures occur in state (i). We use lifetimes to represent failure modes for states (ii), (iv), and (v), and we use probabilities to represent failure modes for state (iii). The heat exchangers include distributions for specific events that may occur in multiple operating modes, such as tube leaks; in these cases, the cumulative running time accounts for all standby and online states. As stated in Section 3.3.5.2.1, we use Hale and Arno for condenser reliability data; this source provides the mean time between failures without consideration for whether the component has been operating for less than an hour or more than an hour. Therefore, we use both online states (iv) and (v) in determining the time at which a condenser component fails. We assume that failure events occur at the beginning of a time period; that is, if a component's lifetime would expire under continuous operation 30 minutes into an hour-long period based on the dispatch provided, we assume that the component is down for the entire time period, rather than for only the last thirty minutes.

A repair event begins immediately after a component failure. During repair events, we assume that plant power cycle thermal capacity and efficiency is reduced by a specified fraction for each component. Repair rate data for most of the components in this maintenance model are sourced from IEEE, an IEEE best practices publication. Condenser repair rates come from a survey by Hale and Arno. This survey includes operational and maintenance data on power generation components collected over a minimum of five years across 40 sites. The U.S. Army converted this survey into a technical report to support decisions requiring reliability metrics (Department of the Army). These sources provide the repair rate as a mean time to repair. We simulate repair times using an exponential distribution with the inverse of mean time to repair as the rate parameter. A constant is added to this repair time to allow for the time required for the system to sufficiently cool down before repairs may begin. Required cooldown times are sourced from interviews with CSP plant operators.

If a repair event takes place for any part of a time period, we assume the capacity and/or efficiency degradation is in effect for that entire time period; or, no operation takes place if the mandatory shutdown capacity or shutdown efficiency limit is reached. For example, if the period length is one hour and a repair event starts in period 5 and lasts for 2.2 hours, the component is not operational in periods 5, 6, or 7. After it is repaired, we treat it as new, i.e., we reset the the hazard rate to one and remove the capacity degradation due to that component.

If multiple components have failed, we assume the effects of capacity and efficiency degradation are additive. As an example, if one failed component reduces the maximum thermal power consumption of the power plant by $10 \%$, and a second component causes a 5\% reduction if it fails in isolation, the cycle thermal power capacity is reduced by $15 \%$ when both components are under repair. If the sum of reductions due to failures meets or exceeds the mandatory shutdown capacity, then we assume the plant's capacity is zero, and no operation takes place for that time period. Similarly, if the combined reductions meet or exceed the no-restart capacity, no operation takes place until all components are repaired. We assume that any number of repairs may take place in parallel.

Components with redundancy are an exception to how repair events are treated. Aguilar et al. describe the concept of 
redundancy in a utility plant. Redundancy enables plant operation to continue in the event of planned maintenance or a component failure by incorporating backup components that are able to perform the same function as a primary component. Active redundant components continue to operate in parallel and passive redundant components are in a standby state waiting to be used when necessary. We model the CSP plant with passive redundancy for all the water pumps. When a pump fails, the standby pump begins operating after a specified transition time (modeled as a short "repair time" for ease of execution in the simulation).

\section{Cycling and ramping effects}

Cycling and fast ramping can cause adverse effects on components in a power cycle. Dispatch decisions include hot starts, warm starts, and cold starts, any of which, when invoked, constitute cycling. Under normal operations, $(\mathcal{R})$ determines whether the plant is in standby mode at the start of power generation. $(\mathcal{R})$ classifies the event as a hot start if the plant is in standby mode. When the system restarts after the conclusion of a repair event, $(\mathcal{S})$ treats the event as a warm start if the repair event's duration is less than a specified threshold, and as a cold start otherwise. Fast ramping is an increase in power output between two discrete time periods that exceeds a given threshold. We assume fast ramping causes accelerated wear and tear to each component, proportional to its hazard rate.

Kumar et al. describe the impact of cycling on failure rates and the accelerated rate of component wear and tear due to ramping. The former is given as a percentage increase in the failure rate per start, while the latter is given as a multiple of normal wear and tear. Failure modes that have lifetimes are simulated as a nonhomogeneous process as follows:

1. Initial lifetimes (i.e., the operating time to failure without considering ramping and cycling) are randomly generated according to a gamma distribution, using specified shape and scale parameters for each component.

2. Hazard rates (i.e., the rate of degradation) are initialized to a value of one for each new component.

3. Hazard rates are increased by a given hot-start penalty for the component when a hot start occurs. This change to the hazard rate lasts until the component fails and is then repaired. An analogous penalty is applied to warm and cold starts. We fit the 25th, 50th, and 75th percentiles for the penalties associated with hot, warm, and cold starts in Kumar et al. to bounded Johnson distributions, and, for each plant instance, we generate a common random percentile from these distributions to obtain the cycling penalties for the plant. The penalties in Kumar et al. denote an absolute increase in expected forced outage rate (EFOR), and present a baseline assumption of $2 \%$; therefore, the relative increases in hazard rate we implement are scaled up by a factor of 50 from those presented by the authors.

4. Lifetimes of components are reduced when the plant is operating based on the following conditions:

A. When the ramping threshold is not exceeded (i.e., the amount of increase in electrical energy generation this period compared to last period is less than the threshold), the life of the component is reduced by the product of the period length (e.g., 1 hour) and the hazard rate, as shown below:

$$
\text { lifetime } \leftarrow \text { lifetime }- \text { period_length } * \text { hazard_rate }
$$

B. When fast ramping occurs, the lifetime is reduced by the product of the ramping penalty, the period length, and the hazard rate, as shown below:

$$
\text { lifetime } \leftarrow \text { lifetime }- \text { ramping_penalty } * \text { period_length } * \text { hazard_rate }
$$

\section{Preventative maintenance events}

A preventative maintenance event occurs when the plant has operated for a specified number of hours. No plant operations take place during these events, which have a fixed duration. For example, a plant may be off-line for a week to perform preventative maintenance after every 5,000 hours of operation. Ramping and cycling do not affect the operating time remaining before the next preventative maintenance event. The results from the maintenance event can be defined as returning certain components to "good as new," i.e., resetting the lifetime and hazard rate to original conditions, or alternatively, reducing the failure rate by some amount determined as input. 
Kumar et al. note that EFOR increases from the baseline only when the number of start exceeds of some predetermined number. As a conservative measure, we apply the penalty to the hazard rate of each component upon every start. Then, when maintenance occurs, we reduce each component's hazard rate by the penalty associated with the expected number of starts to take place over the interval between maintenance events, with the exception that we never reduce the hazard rate below one.

\subsection{Integrated rolling solution}

This section describes the rolling horizon solution method we use to obtain an optimal dispatch schedule over an operating horizon of one year, while accounting for reliability and scheduled maintenance events. This method was first developed by Le and Day and has been used in a variety of applications, including network revenue management (Ciocan and Farias), queueing management (Shah and Wischik), and microgrid design (Silvente et al.). Figure 11 illustrates the development of a year-long solution by optimizing over, and simulating downtime for, 24-hour increments in a rolling horizon that resolves an instance of the dispatch model $(\mathcal{R})$ with added capacity and effectiveness constraints, when failures occur, and shifts by 24 hours after the first 24 -hour increment is finalized.

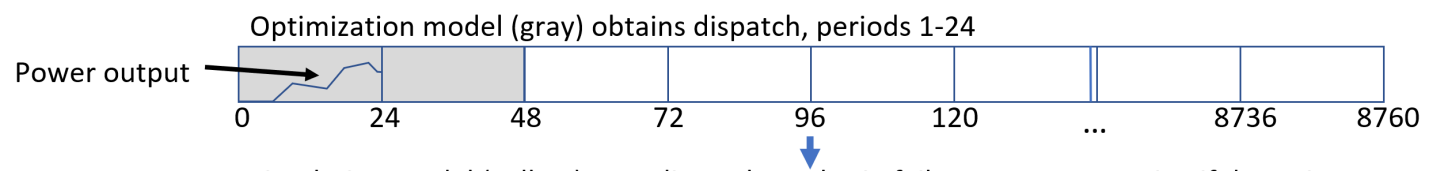

Forced downtime Simulation model (yellow) uses dispatch to obtain failure events, stopping if downtime

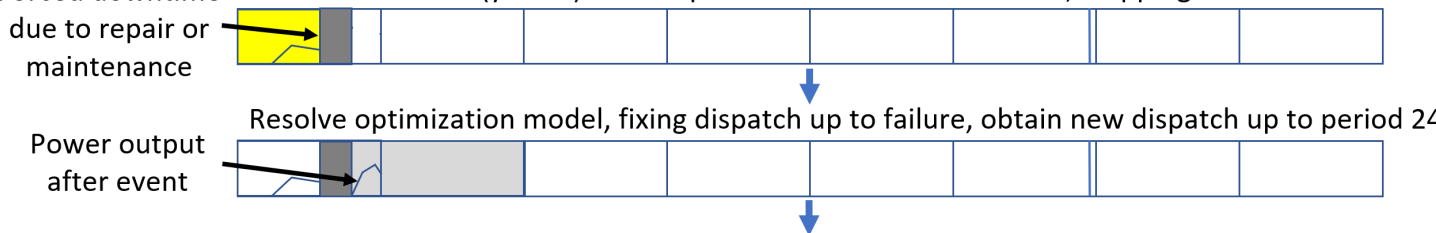

No new repair Simulation model uses dispatch to obtain failure events up to period 24

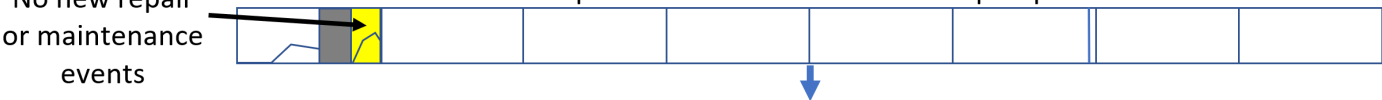

If no new events up to period 24, optimization model obtains dispatch, periods 25-48

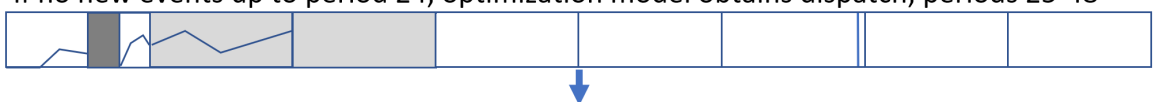

Effective capacity Simulation model uses dispatch to obtain failure events, stopping if downtime reduction due to failure

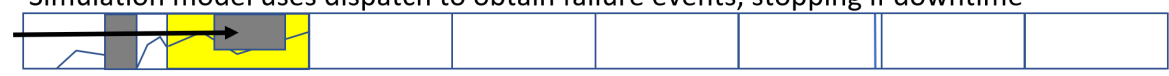

Figure 11. Solution development by the optimization model $(\mathcal{R})$ and simulation model $(\mathcal{S})$, which obtain optimal dispatch and simulated failure and maintenance events over a rolling horizon.

In our method, $(\mathcal{R})$ obtains an optimal dispatch solution for the first 24-hour time frame of the operating horizon using inputs from the SAM engineering model. (As described in Section 3.4.1, $(\mathcal{R})$ solves a 48-hour horizon, but only the first 24 hours are saved.) Next, $(\mathcal{S})$ obtains failure and maintenance events using the 24-hour schedule as input. If $(\mathcal{S})$ results in any capacity reductions, efficiency reductions, or maintenance events, $(\mathcal{R})$ is resolved with variables fixed at their original values up to the first period with a failure or maintenance event. It is also resolved after a repair event concludes. Variables are fixed in $(\mathcal{R})$, using their values determined before using $(\mathcal{S})$, by adding the constraint shown in equation (3.21).

$$
x_{t}=x_{t}^{\text {fixed }} \forall t<\text { failure_period }
$$

where,

New constraints limiting plant capacity and efficiency (shown in equations (3.19) and (3.20) in Section 3.3.5.2.1) are added to $(\mathcal{R})$ beginning with the first period containing a failure or maintenance event. For example, if a component 
fails in period 12 with a resulting $50 \%$ capacity reduction while it is being repaired for four time periods, then a constraint is added to $(\mathcal{R})$ limiting power generation by $50 \%$ for periods 12 through 15 . Additionally, thermal power consumption is fixed, using the original schedule, through period 11 (the period before the failure). A new dispatch schedule for periods 12 through 24 is obtained with these added constraints. If another simulated event occurs before the time frame ends, then the optimization model is again resolved with added constraints. This process continues until the time frame ends. At this point, the 24-hour solution is provided to the engineering model, which creates the final solution. Then, we start the process again with the next time frame (i.e., periods 25-48). This process continues until the end of the operating horizon.

\subsection{Inputs}

The inputs to the cycle simulation model include the following categories:

- power cycle composition data, which describes the number of each type of components in the CSP plant;

- power cycle parameter data, which describes plant-wide attributes, such as the interval between planned maintenance events and the current plant state;

- dispatch data, which include time-series inputs for electricity generation and thermal power consumption;

- policy data, which include thresholds for halting operation either immediately after a component failure or after the dispatch data indicates a shutdown; and,

- simulation parameters, which includes random number seeds, the number of replicates to simulate, and the length of a time period.

Details on the full collection of specific inputs and outputs for model $(\mathcal{S})$ are available in the readme file for the cycle library, which can be found in the GitHub repository at the following link: https://github.com/NREL/ dao-tk/tree/develop/libcycle.

\subsection{Outputs}

The main deliverables of this simulation are (i) a collection of stochastic realizations of failure, repair, and maintenance events over the operating time horizon, and (ii) a time series of plant capacity and efficiency that account for any failed components. Each realization includes an optimized dispatch that accounts for power plant capacity and efficiency reductions from maintenance and repairs based on the operating strategy. The output of $(\mathcal{S})$ can be used to evaluate the impacts of different operating strategies. Section 3.3.5.3 describes a parametric study that varies dispatch penalties and operating strategies to determine the impact on electricity sales, number of start ups, and the amount of ramping.

\subsubsection{Results}

This section describes a the results of a case study that evaluates the impact that different operating strategies have on failures in a CSP plant using our methodology of a maintenance and failure simulation model integrated with an optimized dispatch model. Section 3.3.5.3.1 defines the design parameters of the CSP plant and the corresponding assumptions. Section 3.3.5.3.2 details the factors and response variables of the experiment. Section 3.3.5.3.3 presents an analysis of the results of the experiment.

\subsection{CSP plant modeled}

For this experiment, we use a CSP power tower with molten-salt storage as defined in SAM. We evaluate $120 \mathrm{MW}$ CSP plant located in Daggett, California, with a solar multiple of 3.0 and 15 hours TES. We use a 30-year period by rerunning the same one-year instance of SAM multiple times and saving the component states throughout. This time frame is used to evaluate long-term wear and tear due to dispatch decisions. We use a pricing profile that varies during the day and by season based on a proposed power plant near Rice, California (see Figure 12). The pricing profile changes the multiplier on the price at which the plant can sell electricity throughout the day and by season. 


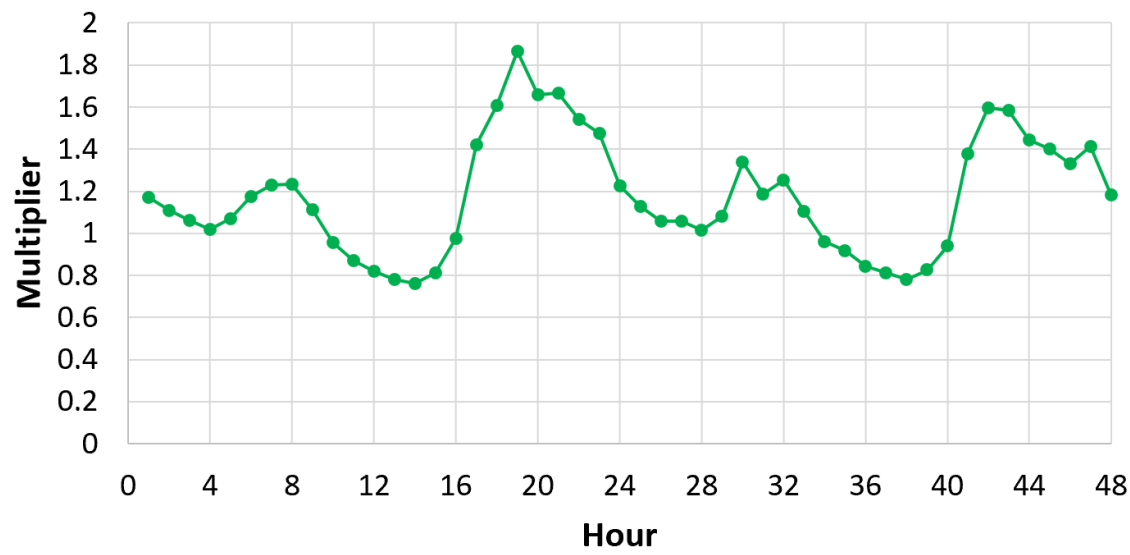

Figure 12. The pricing profile used with the repeated experiment, showing the first $\mathbf{4 8}$ hours of the one-year schedule which also varies by season.

Here, we describe assumptions and parameters for this experiment. To focus on the impact that operating decisions have on failures, we assume that there is no preventative maintenance conducted during the time horizon of the experiment. We exclude the costs of repairs due to a lack of empirical data in the literature and because we assume that revenue losses from decreased capacity and efficiency far outweigh the costs of repairs. When we begin the power plant simulation, we randomly generate component lifetimes and probabilities and set the hazard rates to 1.0. We do not use a warm-up period because we are simulating 30 years of a plant lifetime. All repairs result in generating a new component lifetime or probability with respect to the failure mode; however, the component retains the lifetimes and probabilities for all other failure modes. For example, performing a repair after failing to start does not impact the lifetime remaining. We assume heliostats never fail and that there are no losses due to dust and dirt. This choice is made so that there are no effects from heliostats on the experiment results. The dispatch is resolved when a failure occurs and when a repair concludes.

We use the gas combined cycle plant data from an NREL report concerning power plant cycling costs (Kumar et al.) as our source for ramping thresholds and multipliers. This report does not have data specific to CSP plants. The ramping penalty is applied as a multiplier (between $1.2 \mathrm{x}$ and $4 \mathrm{x}$ the hazard rate) when ramping is between 110$200 \%$ of the defined threshold. The ramping penalty is only applied during the period in which fast ramping occurs. Because Kumar et al. only present penalties for starting the power cycle, we do not apply any penalties when the power block is shutdown.

\subsection{Design of experiments}

We conduct a design of experiments using several factors and levels, as shown in Table 12, and generate solutions for a 30-year time frame. The goal is to determine the best operating policy to maximize sales when considering lost capacity and efficiency due to failures. Though electricity sales are the primary response of interest, we also examine the number of power cycle starts and the number of failures. The experiment design includes every level of the cycling cost factor with the low ramping penalty level. We also evaluate the two ramping penalty levels using several cycling cost levels. We use 30 replicates for statistical analysis. Each replicate uses a different random number seed in $(\mathcal{S})$ to model new instances of failure mode lifetimes and probabilities.

The cycling cost and ramping penalty factors are derived from the NREL cycling cost study by Kumar et al. We vary the cycling cost, the $(\mathcal{R})$ objective function penalty due to power cycle startups, between $\$ 0$ and $\$ 100,000$ per startup. The lowest cycling cost level, $\$ 0$ per startup, represents no penalties for startups in $(\mathcal{R})$. The highest cycling level, $\$ 100,000$ per startup, represents four times the highest value for any type of power plant in the NREL study. The $\$ 6,250$ value is near the median warm start cost for a $120 \mathrm{MW}$ gas combined-cycle power plant, and the penalty used in Wagner et al. The combined-cycle power plant is the closest match in operation to a CSP plant in 


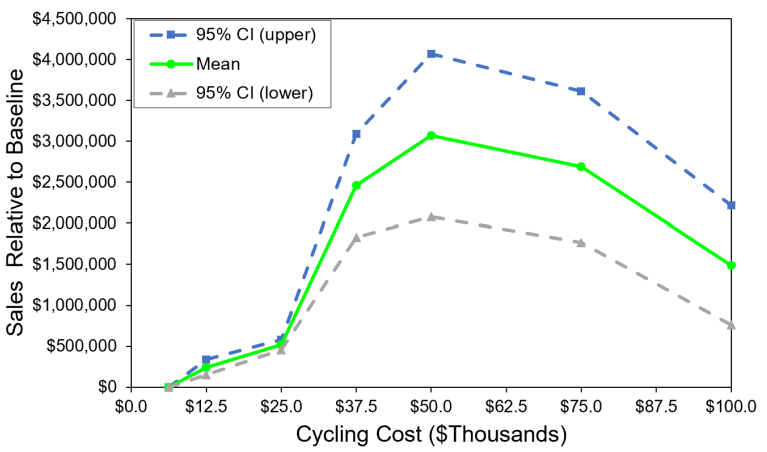

(a) Sales by cycling cost

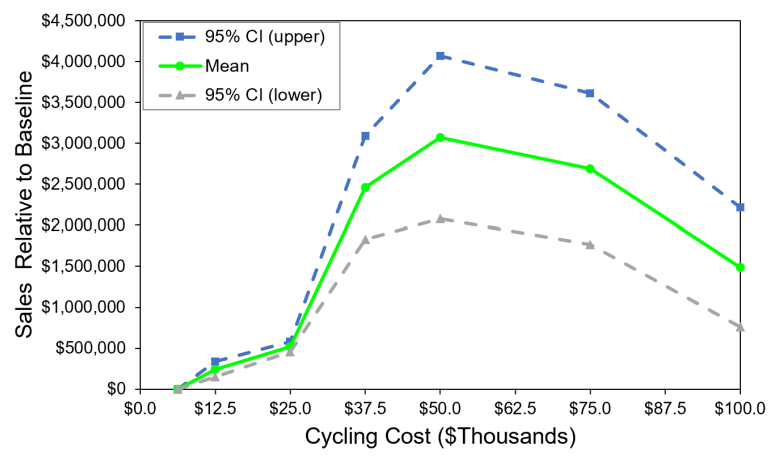

(b) Failures by cycling cost

Figure 13. Results of sales and failures by cycling cost, shown in parts (a) and (b), respectively. As the cycling cost is increased, there is a point when sales are maximized and the number of failures stop declining, on average.

the NREL cycling cost study. We include other levels spaced between the low and high values. We vary the ramping penalty, the $(\mathcal{R})$ objective function penalty due to ramping, between $\$ 0$ and $\$ 10$ per MW at the low and high levels of the cycling cost factor. The low value represents no ramping penalty and the high value is greater than the highest ramping cost for a power plant in the NREL study.

\subsection{Results analysis}

This section reviews the results from the experiment. We analyze the factors graphically and perform a paired t-test to determine which cycling cost results in the most improvement in sales, when considering component availability. We can use a paired t-test because each replicate consists of the same sequence of random numbers that determine component lifetimes and probabilities of failure. The paired t-test consists of subtracting the 30-year sales value for each cycling cost level and determining if the average difference contains zero using a significance of $\alpha=0.05$.

We find that increasing the cycling cost is significant up to about $\$ 50,000$ per startup. The next highest cycling cost tested, $\$ 75,000$ per startup, is not statistically different than $\$ 37,500$ or $\$ 50,000$ per startup. Part (a) of Figure 13 displays sales by cycling cost, scaled to the results from using a cycling cost of $\$ 6,250$, which is near the value used by Wagner et al. This figure shows that sales increase as the cycling cost is increased, due to less downtime from failures, until a threshold is reached where the cycling penalty is too restrictive. At this point, failures continue to decline, as shown in part (b) of Figure 13, but the high cycling penalty also precludes earning profit by reducing the number of startups. The ramping penalty is not significant for both sales and failures, likely because we are using an hour-fidelity model which does not need to ramp beyond the allowed threshold.

The results from our study also confirm that the integrated models are operating as expected. Increasing cycling costs results in fewer startups and increasing ramping penalties results in less ramping (not shown). We also find that the number of startups has a significant impact on the number of failures, as shown in Figure 14. More startups result in more failures, on average.

The results of these experiments may be specific to the CSP plant design, the location, and the component failure and repair distributions we use. We provide this analysis as an example of the type of insights that could be derived using the integrated dispatch optimization and failure simulation models. Future users should employ their specific power plant designs, locations, pricing profiles, and failure and repair rates.

\subsubsection{Conclusion}

This section presents a methodology for considering power plant maintenance and component failures in conjunction with dispatch optimization. We integrate a failure simulation with a dispatch MIP and evaluate the impact that 


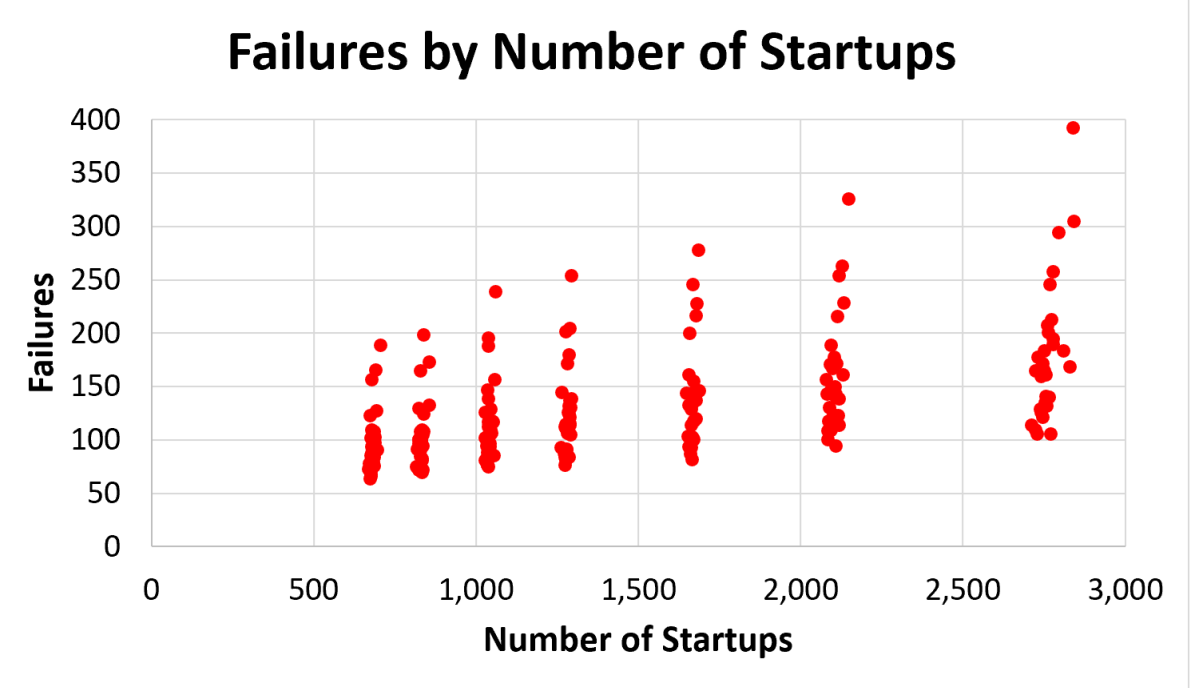

Figure 14. The number of failures are directly related to the number of startups. The four groups of startups are a result of the four cycling costs, with higher cycling costs associated with fewer startups.

different operating strategies have on electricity sales and component failures in a CSP plant. Our results show that the number of startups (driven by the cycling cost penalty) significantly affect electricity sales when considering component failures using a 30-year time horizon and a CSP plant with a solar multiple of 3.0 and 15 hours of TES. Applying our methodology of integrating a reliability simulation with an optimized dispatch model to a CSP plant resulted in finding a cycling penalty that increased average sales by about $\$ 3$ million over 30 years, compared to the penalty used in prior research (Wagner et al.).

The insights gained by studying the affect that different operating policies have on long-term reliability can support higher level decisions such as system design. For example, NREL's SAM can be used to optimize a CSP plant design for a given location and plant size. SAM uses dispatch optimization to help evaluate different design choices. But, that optimal design may be using dispatch policies that could have a long-term negative effect on the availability to generate power and the costs of maintenance. The profitability of a design could be over estimated without considering these losses. Integrating a maintenance and failure simulation with an optimized dispatch model takes into account potentially harmful operating policies.

Future work will incorporate repair costs for components, different power plant designs, and alternate locations and pricing profiles. Including parts and labor may add a significant expense to some operating policies. Here we only consider lost revenue due to downtime from failures. Additionally, this model can be incorporated into a larger problem that optimizes a CSP plant design that accounts for component availability.

\subsection{Dispatch optimization}

\subsubsection{Deterministic formulation}

The economic viability and widespread implementation of CSP technologies are strongly tied to their ability to extend their diurnal operational characteristics across peak demand time periods and during periods when solar energy is curtailed due to the sun setting or cloud cover (Jorgenson, Denholm, and Mehos). Thermal energy storage is an enabling technology which can amass the energy captured by the receiver as a reserve for dispatch at a later, more favorable time. In fact, TES integration enables CSP to be a dispatchable renewable resource whose economics are enhanced by both improved utilization of the power cycle and an ability to shift power production to better coincide with peak demands and high-value-electricity time periods (Turchi et al.).

The maximum storage capacity of the TES system is determined during a plant design process that considers several 
factors including the thermal power rating of the solar field and power cycle subsystems, plant location, project economics, and the desired capacity factor, which is defined as the quotient of total annual electrical energy production and the electrical energy production should the plant operate continuously at rated power output. Thermal energy storage sizing also depends on the operational scheme. For example, a plant that intends to operate primarily during high-revenue morning or evening periods while reducing production during daylight hours requires more TES capacity than a plant with an identical capacity factor that generates power during all daylight hours. CSP plants that target dispatch during high-revenue periods operate differently than those that minimize the average cost of energy. The former relies more extensively on a carefully planned dispatch schedule that anticipates the timing and level of thermal power production in the solar field, energy consumption for receiver and plant start up, and the charge state of TES over time. Formal optimization methods can determine the dispatch profile that maximizes electricity sales revenue over a particular time horizon given a specific system configuration, expected solar resource, pricing or time-of-dispatch (TOD) schedule, and operational constraints - a process referred to as dispatch optimization.

The intelligent dispatch of stored energy can greatly enhance the value of electricity by providing firm capacity and ancillary services, and by generating electricity during time periods in which rates are especially high (Denholm and Mehos). Dispatch optimization involves the manipulation of the timing and rate at which electricity is generated by the power cycle and captures both physical processes and time (Pruitt, Braun, and Newman). This section presents a foundational methodology, implementation, and publicly available tool for simulating CSP power tower systems with optimized dispatch. The method expands on previous work by directly incorporating formal optimization techniques into the SAM (Blair et al.) simulation software, for which previous research has relied on heuristics or on optimizing dispatch using simulation output a posteriori as optimization model input. SAM assesses CSP performance, simulating renewable technologies including CSP, wind, geothermal, photovoltaic, biomass, solar hot water, and generic systems. The software is free to download and use, and the tools developed in the current work are freely available (National Renewable Energy Laboratory). Each technology can be paired with a financial model to evaluate the economic performance of a project within particular market, incentive, and cost environments.

\section{Related Work}

Optimization modeling has been applied to many types of energy systems, e.g., (Pruitt, Braun, and Newman) who retrofit an existing building and determine a corresponding dispatch strategy, and (Perera et al.) who examine multiple objectives in optimizing stand-alone hybrid energy systems, also with the corresponding dispatch. Other authors examine only dispatch, e.g., (Nosrat and Pearce), who apply a simulation model to a hybrid photovoltaic and trigeneration power system to decrease waste from excess heat, while (Pandžić, Kuzle, and Capuder) formulate an optimization model (a mixed-integer linear program, like ours) that combines both dispatchable and intermittent power, the latter as a result of a virtual plant, to maximize profits. Similarly, (Martinez-Rojas et al.) develop an optimization model that dispatches wind, but, in contrast to the previous work, theirs focuses on minimizing active power losses in the system while constraining reactive power; the model is solved heuristically. Thorin, Brand, and Weber, Cho et al. and Fürsch et al. operate in a market environment (as does Pandžić, Kuzle, and Capuder), the former for a unit commitment problem, applying an exact approach (i.e., Lagrangian Relaxation) to a mixed-integer program; Cho et al. optimize a combined cooling, heating, and power system to optimize the tradeoffs between system cost, energy production and emissions, and test their model on a variety of geographic sites in the U.S. with differing weather conditions; Fürsch et al. examine the expansion of a power network and the corresponding dispatch strategies in Europe; using an optimization model which combines both investment and dispatch decisions, they conclude that even optimal grid extensions, coupled with capital cost reductions for renewable technologies, leads to significantly higher overall average electricity system costs over a time horizon of three to four decades. Parisio et al. use model predictive control within an optimization (mixed-integer programming) framework in which the goal is to minimize costs subject to microgrid system constraints such a capacities, minimum up- and down-times, and start-up and shutdown requirements. They test instances of their model on an experimental microgrid in Greece. Zheng et al. provide a review of bio-inspired optimization of sustainable energy systems. These works examine problems similar to ours in that dispatch policies are considered, some even using the mathematical framework in this section. However, none of these examines concentrating solar power in particular, with its own sets of objectives and rules. This section next discusses the research specific to power tower technology. 
Simulation is used to predict the total electrical energy production from an existing or previously designed CSP plant over its lifetime in order to evaluate the financial return on investment, the cost of energy, the environmental (mitigation) impact, or some other measure of interest. The standard method for CSP simulation requires calculation of plant behavior over a time horizon (typically, one year with one-hour time steps) (Hirsch et al.), and it develops a picture of long-term energy production by sequentially modeling performance at relatively short time steps compared to the overall time window of interest (e.g., hourly calculations to establish lifetime metrics). CSP systems are primarily constrained by immediate concerns, such as component or subsystem operational states, conservation of mass and energy, and heat transfer, thermodynamic, or thermo-mechanical principles.

The previous dispatch approach implemented in SAM uses a simple heuristic that allows the user to specify requirements before thermal storage can be dispatched; this heuristic does not consider the expected future thermal energy production, TES charge state, or price at which electricity can be sold, but instead determines the operational state of the power cycle based on the current TES charge state and the hour of the day. The heuristic can improve plant production during high-value hours as exemplified by SAM or Guédez et al., but can ultimately decrease the utilization of the solar field throughout the year because of TES over-charge situations.

By contrast, this work adopts a formal approach by formulating the problem as a mixed-integer program (MIP) that leverages state-of-the-art modeling languages and solvers ((Fourer, Gay, and Kernighan; IBM)) to make the solution of a mathematical problem containing thousands of variables and constraints tractable.

Madaeni et al. present a simplified approach for determining an optimal dispatch profile while implementing MIP techniques. The authors use SAM to generate an hourly thermal power production profile throughout the year that is considered as fixed input to the MIP model originally outlined in (Sioshansi). This approach factors in the simulated performance of the solar field, but omits interactions between the solar field and thermal storage or the power cycle. The latter subsystems are modeled as part of a MIP that determines the TES state of charge and electricity production from the cycle. This method improves tractability by reserving the detailed model to generate fixed input while utilizing a simplified energy balance model to characterize TES charge and power cycle generation. Furthermore, Madaeni et al. employ a rolling time horizon methodology in which they consider a 48-hour time horizon, updated every 24 hours. This work largely adopts this approach, but importantly, uses the optimized schedule to control operational decisions within SAM's detailed simulation model, whereas the Madaeni et al. work uses the results from the MIP as the actual estimate of plant production throughout the year.

\section{Goals of this work}

Dispatch optimization improves the profitability of existing or planned CSP facilities, but it is also of great interest to policymakers and researchers who seek to better understand the projected performance of CSP systems under various deployment and grid operations scenarios. However, previous work (cf. (Denholm and Mehos; Madaeni et al.; Sioshansi)) considers dispatchability from the perspective of grid integration in which CSP systems are designed at an energy-flow and system sizing level to assess suitability for meeting grid and market demands. The contribution of this work is its evaluation of the relationship between optimal dispatch profiles and technology design. Accordingly, this work fills the gap between prescriptive grid-level models on the one hand that indicate desired technology performance subject to high-level operational requirements (e.g., plant start up, maximum energy generation) and descriptive performance simulations on the other hand whose primary concern is to dynamically synthesize expected plant productivity and financial return given specific component or subsystem thermo-mechanical performance expectations. SAM develops these estimates using annual "macro-simulations" that consist of thousands of sequential "micro-simulations" within a time series, and the plant behavior at any given time step may depend on the state of the system in the previous time step(s).

The MIP in SAM operates under the following assumptions: (i) solar field thermal production over time is calculated using a simplified "forecast" model and provided as a fixed input to the dispatch model, and (ii) power cycle efficiency depends linearly on thermal input to the cycle and on the ambient temperature, and these efficiency corrections can be implemented as independent terms (see Section 3.4.1.6). Dispatch optimization enables investigation of detailed plant performance issues that are too complex to be easily represented in an energy-balance MIP model. For example, the mechanical stress associated with frequent thermal cycling of power generation equipment may 
lead to an increase in the frequency of required maintenance (Guédez et al.). A detailed model can capture these thermo-mechanical impacts when the plant control is influenced by optimized dispatch scheduling.

\section{Operating Considerations}

The plant dispatch schedule determines the timing and production level from the power cycle (turbine, generator, condenser, and associated equipment). During operation, the power cycle consumes stored thermal energy from the TES system. Thermal energy storage is charged using high-grade thermal energy that is generated by the solar field during daytime operation, and energy generation is affected by the optical and thermal efficiency of the solar field, by the intensity of the available solar resource, and by the operational state of the solar field. Receiver and power cycle start-up sequences are not necessarily coordinated, so both systems may operate independently with shared interest only in the energy state of the TES system. In some cases, the receiver must curtail energy generation to avoid over-charging thermal storage (thus wasting solar energy).

Before the power cycle or receiver can produce electricity or thermal energy, respectively, start-up requirements must be satisfied, including both a minimum start-up period and a minimum energy state requirement which are surrogates for temperature considerations. In the latter case, the plant equipment cools during shutdown periods and must overcome the system's thermal inertia to begin generating steam that powers the turbine. Likewise, the receiver consumes energy as it heats up and must complete a start-up procedure before producing useful thermal energy. Furthermore, turbine and heat exchanger equipment manufacturers limit the maximum rate of temperature increase during start-up to avoid thermal stress and mechanical failure risks. Both the energy and duration start-up requirements must be met before equipment can begin producing power. These requirements are implemented as a constraint on the maximum energy delivered for start-up during any given time period. Although the duration of start-up must last for at least a minimum number of time steps, longer start-up durations are allowed in practice based on energy availability, and the model must provide this flexibility.

Two start-up scenarios are considered for the power cycle: (i) cold start-up, which occurs when the power cycle has shut down for any period of time and seeks to restart; and (ii) hot start-up, which occurs when the power cycle has been in standby mode and seeks to restart. Cold start-up requires an additional energy contribution and incurs more component wear and tear, whereas hot start-up can happen immediately (from the perspective of the hourly model).

Standby is a mode of operation in which a small (but non-trivial) amount of thermal energy is consumed during each time period to maintain the power cycle and/or receiver equipment in a hot state, ready to quickly ramp up for electricity generation; however, no electricity is produced in standby mode. Consequently, maintaining the power cycle in standby mode is of value if multiple start-up cycles are anticipated over a relatively short time span, or if the energy penalty or ramp rate requirement for start-up is sufficiently severe to justify the small rate of energy consumption by the power cycle.

The receiver can also operate in a standby mode during cloudy periods to avoid the full start-up procedure. In standby, salt from the cold storage tank is pumped through the receiver, and the flow is diverted back into the cold tank where the fluid temperature can decay at a rate that corresponds to the thermal losses from the receiver. Finally, the model accounts for receiver shutdown energy consumption in which the heliostat field provides sufficient energy to allow the salt to drain out without freezing before the solar field ends operation for the day. The draining procedure requires approximately fifteen minutes while sunlight is still available, and this effect is modeled as the consumption of $25 \%$ of the hourly energy used at the minimum receiver production rate.

\subsubsection{Mathematical Formulation}

The parameters, sets, variables, objective function, and constraining relationships are described in this section. The model takes the parameters and sets as given and determines values for the decision variables to maximize an objective function while adhering to the constraints. Some parameters and all variables are subscripted with time $t$, indicating the time-varying nature of the decisions. 


\section{Parameters and Sets}

The following MIP, $(\mathcal{R})$, requires the initial operational state of the system, the collector field and receiver energy generation profile, the expected cycle conversion efficiency profile as a function of ambient temperature and thermal input, and the energy price or tariff profile (Table 13). (Initialization parameters used to set variable values at $t=0$ follow variable notation and are not included here.)

Table 13. Parameters and sets used in $(\mathcal{R})$.

\begin{tabular}{|c|c|c|}
\hline $\begin{array}{l}\text { Symbol } \\
\text { Sets }\end{array}$ & Units & Description \\
\hline $\mathcal{T}$ & & Set of all time steps in the time horizon, $T=|\mathcal{T}|$ \\
\hline \multicolumn{3}{|c|}{ Time-indexed parameters } \\
\hline$Q_{t}^{i n}$ & $\mathrm{~kW}_{t}$ & ${ }^{*}$ energy generated by the solar field in time $t$ \\
\hline$P_{t}$ & $\$ / \mathrm{kW}_{e}-\mathrm{hr}$ & electricity sales price in time $t$ \\
\hline$\dot{W}_{t}^{n e t}$ & $\mathrm{~kW}_{e}$ & net power production upper limit in time $t$ \\
\hline$\dot{W}_{t}^{\min }$ & $\mathrm{kW}_{e}$ & minimum net power production in time $t$ \\
\hline$\eta_{t}^{c}$ & - & normalized condenser parasitic loss in time $t$ \\
\hline$\gamma_{t}$ & - & exponential time weighting factor; $\Gamma^{(t)}$, where $\Gamma \approx 0.99$ \\
\hline$\Delta_{t}^{r s}$ & - & *estimated fraction of time step $t$ used for receiver start-up \\
\hline$\eta_{t}^{a m b}$ & - & cycle efficiency adjustment factor in time $t$ \\
\hline \multicolumn{3}{|c|}{ Steady-state parameters } \\
\hline $\bar{P}$ & - & mean sales price $(\$ / \mathrm{kW}-\mathrm{hr}) ; \sum_{t \in \mathcal{T}} P_{t} / T$ \\
\hline$\eta^{d e s}$ & - & cycle nominal efficiency \\
\hline$\eta^{p}$ & - & * slope of linear approximation of power cycle performance curve \\
\hline$\tau$ & $\mathrm{hr}$ & frequency of optimization problem execution \\
\hline$E^{u}$ & $\mathrm{~kW}_{t}$-hr & energy storage capacity \\
\hline$E^{r}$ & $\mathrm{~kW}_{t}$-hr & required energy consumed to start receiver \\
\hline$E^{c}$ & $\mathrm{~kW}_{t}$-hr & required energy consumed to start cycle \\
\hline$E^{h s}$ & $\mathrm{~kW}_{t}-\mathrm{hr}$ & heliostat field startup or shutdown parasitic loss \\
\hline$Q^{u}$ & $\mathrm{~kW}_{t}$ & cycle thermal power capacity \\
\hline$Q^{\prime}$ & $\mathrm{kW}_{t}$ & minimum operational thermal power input to cycle \\
\hline$W^{u}$ & $\mathrm{~kW}_{e}$ & cycle electric power rated capacity \\
\hline$W^{\prime}$ & $\mathrm{kW}_{e}$ & minimum electric power output from cycle \\
\hline$\dot{W}^{h}$ & $\mathrm{~kW}_{e}$ & heliostat field tracking parasitic loss \\
\hline$\dot{W}^{b}$ & $\mathrm{~kW}_{e}$ & power cycle standby operation parasitic load \\
\hline$\dot{W}^{r s b}$ & $\mathrm{~kW}_{e}$ & tower piping heat trace parasitic loss \\
\hline$Q^{r u}$ & $\mathrm{~kW}_{t}$ & allowable power per period for receiver start-up \\
\hline$Q^{r l}$ & $\mathrm{~kW}_{t}$ & minimum operational thermal power delivered by receiver \\
\hline$Q^{r s d}$ & $\mathrm{~kW}_{t}$ & required thermal power for receiver shut-down \\
\hline$Q^{r s b}$ & $\mathrm{~kW}_{t}$ & required thermal power for receiver standby \\
\hline$Q^{c}$ & $\mathrm{~kW}_{t}$ & allowable power per period for cycle start-up \\
\hline$Q^{b}$ & $\mathrm{~kW}_{t}$ & standby thermal power consumption per period \\
\hline$L^{r}$ & $\mathrm{~kW}_{e} / \mathrm{kW}_{t}$ & receiver pumping power per unit power produced \\
\hline$L^{c}$ & $\mathrm{~kW}_{e} / \mathrm{kW}_{t}$ & cycle Heat Transfer Fluid (HTF) pumping power per unit energy consumed \\
\hline$C^{r s u}$ & $\$$ & penalty for receiver start-up (from 0 ) \\
\hline$C^{r h s}$ & $\$$ & penalty for receiver start-up (from hot standby) \\
\hline$C^{\operatorname{csu}}$ & $\$$ & penalty for cycle start-up (from 0) \\
\hline$C^{\text {chs }}$ & $\$$ & penalty for cycle start-up (from hot idle) \\
\hline$C^{\delta W}$ & $\$ / \mathrm{kW}_{e}$ & penalty for any positive change in electricity production \\
\hline$\Delta$ & $\mathrm{hr}$ & time step duration \\
\hline
\end{tabular}




$\begin{array}{lcl}\text { Symbol } & \text { Units } & \text { Description } \\ \Delta^{J} & \text { hr } & \text { minimum duration of receiver start-up in period } \\ \alpha & \$ & \text { conversion factor between unit-less and monetary values } \\ \mathbb{M} & & \text { a sufficiently large number }\end{array}$

\section{Variables}

The variables (see Table 14) describe energy (thermal $k W_{t}-h r$ or electric $k W_{e}-h r$ ) states and power flows (thermal $k W_{t}$ or electric $k W_{e}$ ) in the system. Note that there is a one-to-one conversion between $k W_{t}$ and $k W_{e}$ units. Continuous variables " $x$," " $\dot{w}$, " " $u$," and " $s$ " representing power and energy relate to the receiver, power cycle, and TES. Binary variables " $y$ ” enforce operational modes and sequencing such that start-up must occur before normal operation, for example.

\section{Objective Function}

The objective maximizes electricity sales, which are represented as the summation over time of the product of electricity price and power generation less parasitic losses. Cost penalties associated with cycle start-up, receiver startup, and change in electricity production between time steps are subtracted from the revenue. Binary variables $y_{t}^{r s d}$ and $y_{t}^{c s d}$ introduce a small penalty that enforces receiver and power cycle shutdown logic, respectively.

$(\mathcal{R})$ maximize

$$
\begin{aligned}
& \sum_{t \in \mathcal{T}}[ \Delta \cdot P_{t}\left(\gamma_{t}\left(1-\eta_{t}^{c}\right) \dot{W}_{t}-L^{r}\left(x_{t}^{r}+x_{t}^{r s u}+Q^{r l} y_{t}^{r s b}\right)-L^{c} x_{t}\right. \\
&-\dot{W}^{h} y_{t}^{r}-\dot{W}^{b} y_{t}^{c s b}-\left(\dot{W}^{r s b}+E^{h s} / \Delta\right) y_{t}^{r s u} \\
&\left.\quad E^{h s} / \Delta \cdot y_{t}^{r s b}-E^{h s} / \Delta \cdot y_{t}^{r s d}\right) \\
&-\gamma_{t}\left(C^{r s u} y_{t}^{r s u p}+C^{r h s} y_{t}^{r h s p}\right) \\
&-\gamma_{t}\left(C^{c s u} y_{t}^{c s u p}+C^{c h s} y_{t}^{c h s p}+\alpha y_{t}^{c s d}+C^{\left.\delta W^{\prime} \dot{w}_{t}^{\delta}\right)}\right. \\
&\left.+\gamma_{t}\left(\bar{P} \Delta x_{t}^{r}+\alpha y_{t}^{r}\right)\right]
\end{aligned}
$$

\section{Constraints}

The relationships among the variables and parameters are established with a set of simultaneous equations and inequalities. These constraints are presented below topically with a brief description.

\subsubsection{Receiver Operations}

Receiver operations constraints include:

\section{Receiver Start-up}

$$
\begin{aligned}
& u_{t}^{r s u} \leq u_{t-1}^{r s u}+\Delta \cdot x_{t}^{r s u} \forall t \in \mathcal{T}: t \geq 2 \\
& u_{t}^{r s u} \leq E^{r} y_{t}^{r s u} \forall t \in \mathcal{T} \\
& y_{t}^{r} \leq \frac{u_{t}^{r s u}}{E^{r}}+y_{t-1}^{r} \quad \forall t \in \mathcal{T}: t \geq 2 \\
& y_{t}^{r s u}+y_{t-1}^{r} \leq 1 \quad \forall t \in \mathcal{T}: t \geq 2 \\
& x_{t}^{r s u} \leq Q^{r u} y_{t}^{r s u} \quad \forall t \in \mathcal{T}
\end{aligned}
$$




$$
\text { if } \begin{aligned}
Q_{t}^{i n} & =0 \text { then: } \\
y_{t}^{r s u} & =0 \quad \forall t \in \mathcal{T}
\end{aligned}
$$

Receiver Supply and Demand

$$
\begin{aligned}
& x_{t}^{r}+x_{t}^{r s u}+Q^{r s d} y_{t}^{r s d} \leq Q_{t}^{i n} \forall t \in \mathcal{T} \\
& x_{t}^{r} \leq Q_{t}^{i n} y_{t}^{r} \forall t \in \mathcal{T} \\
& x_{t}^{r} \geq Q^{r l} y_{t}^{r} \quad \forall t \in \mathcal{T} \\
& \text { if } Q_{t}^{i n}=0 \text { then: } \\
& \quad y_{t}^{r}=0 \quad \forall t \in \mathcal{T}
\end{aligned}
$$

Logic Governing Receiver Modes

$$
\begin{aligned}
& y_{t}^{r s u}+y_{t}^{r s b} \leq 1 \quad \forall t \in \mathcal{T} \\
& y_{t}^{r}+y_{t}^{r s b} \leq 1 \quad \forall t \in \mathcal{T} \\
& y_{t}^{r s b} \leq y_{t-1}^{r}+y_{t-1}^{r s b} \forall t \in \mathcal{T}: t \geq 2 \\
& y_{t}^{r s u p} \geq y_{t}^{r s u}-y_{t-1}^{r s u} \forall t \in \mathcal{T}: t \geq 2 \\
& y_{t}^{r s s p} \geq y_{t}^{r}-\left(1-y_{t-1}^{r s b}\right) \forall t \in \mathcal{T}: t \geq 2 \\
& y_{t-1}^{r s d} \geq\left(y_{t-1}^{r}-y_{t}^{r}\right)+\left(y_{t-1}^{r s b}-y_{t}^{r s b}\right) \forall t \in \mathcal{T}: t \geq 2
\end{aligned}
$$

$(\mathcal{R})$ considers receiver start-up inventory and the criteria that must be satisfied in order for it to produce useful power. Constraint (3.23a) tracks start-up energy "inventory" using an inequality, rather than an equality, to allow inventory to reset to zero in time periods following start-up completion; inventory is naturally maximized by the problem and can only be nonzero for time steps in which the receiver is starting up by Constraint (3.23b). Constraint (3.23c) allows receiver power production only after start-up has been completed or when the receiver was operating in the previous time step. Constraint (3.23d) ensures that receiver start-up mode does not persist while the receiver is operating in power-producing mode by disallowing start-up in the time step following normal power production operation. Constraint (3.23e) ensures that the actual power used for receiver start-up is no more than the ramp rate limit for each time step. Constraint (3.23f) prevents receiver start-up from occurring in time periods with trivial solar resource.

The total power produced by the receiver has an upper bound of the available energy $Q_{t}^{\text {in }}$, and any start-up or shutdown energy consumption detracts from production according to Constraint (3.24a). The receiver can only generate thermal power when it is in power-producing mode (i.e., $y_{t}^{r}=1$ ) by Constraint (3.24b). Constraint (3.24c) is enforced because of molten-salt pump operating limits and heat transfer requirements in the receiver, ensuring that the receiver energy generation must satisfy a minimum threshold. Constraint (3.24d) ensures that the receiver power-producing mode does not persist when no energy is available.

While the receiver is in standby mode, molten salt is circulated between the cold TES tank and receiver, enabling fast restart. A smaller hot start-up penalty is enforced when beginning normal operation from standby mode. Neither standby and start-up modes (Constraint (3.25a)) nor standby and power-producing modes (Constraint (3.25b)) can coincide. Standby mode can persist over time, but must follow time steps in which the receiver was either in standby or power-producing mode (Constraint (3.25c)). Constraints (3.25d) and (3.25e) enforce logic associated with incurring a penalty for receiver start-up from an off or standby state, respectively. Constraint (3.25f) enforces the logic for shut-down from a power producing or standby state. Constraint (3.31a) ensures non-negativity for receiver start-up power consumption and receiver start-up energy inventory. Non-negativity for $X_{t}^{r}$ is ensured via Constraint (3.24c). Constraint (3.31c) enforces binary requirements on the variables associated with generating usable thermal power, receiver start-up, receiver standby, receiver shut down, and receiver start-up penalties. 


\subsubsection{Power Cycle Operations}

Power cycle operation constraints largely mirror those of receiver operations and include:

Cycle Start-up

$$
\begin{aligned}
& u_{t}^{c s u} \leq u_{t-1}^{c s u}+\Delta \cdot Q^{c} y_{t}^{c s u} \quad \forall t \in \mathcal{T}: t \geq 2 \\
& u_{t}^{c s u} \leq \mathbb{M} y_{t}^{c s u} \forall t \in \mathcal{T} \\
& y_{t} \leq \frac{u_{t}^{c s u}}{E^{c}}+y_{t-1}+y_{t-1}^{c s b} \quad \forall t \in \mathcal{T}: t \geq 2 \\
& x_{t}+Q^{c} y_{t}^{c s u} \leq Q^{u} y_{t} \quad \forall t \in \mathcal{T} \\
& x_{t} \leq Q^{u} y_{t} \quad \forall t \in \mathcal{T} \\
& x_{t} \geq Q^{\prime} y_{t} \forall t \in \mathcal{T}
\end{aligned}
$$

Power Supply and Demand

$$
\begin{gathered}
\dot{w}_{t} \leq \frac{\eta_{t}^{a m b}}{\eta^{d e s}}\left(\eta^{p} x_{t}+y_{t}\left(W^{u}-\eta^{p} Q^{u}\right)\right) \quad \forall t \in \mathcal{T} \\
\dot{w}_{t}^{\delta} \geq \dot{w}_{t}-\dot{w}_{t-1} \quad \forall t \in \mathcal{T}: t \geq 2 \\
\text { If } \dot{W}_{t}^{\text {net }} \geq \dot{W}_{t}^{\min } \text { then: } \\
\dot{W}_{t}^{\text {net }} \geq \dot{w}_{t}\left(1-\eta_{t}^{c}\right)-L^{r}\left(x_{t}^{r}+x_{t}^{r s u}\right) \\
\quad-x_{t} L^{c}-y_{t}^{r s u}\left(\frac{\dot{W}^{r s b}}{\Delta}+\frac{E^{h s}}{\Delta}\right) \\
\quad-\dot{W}^{h} y_{t}^{r}-y_{t}^{c s b} \dot{W}^{b} \forall t \in \mathcal{T} \\
\text { else: } \quad \dot{w}_{t}=0 \forall t \in \mathcal{T}
\end{gathered}
$$

Logic Governing Cycle Modes

$$
\begin{aligned}
& y_{t}^{c s u}+y_{t-1} \leq 1 \quad \forall t \in \mathcal{T}: t \geq 2 \\
& y_{t}^{c s b} \leq y_{t-1}+y_{t-1}^{c s b} \forall t \in \mathcal{T}: t \geq 2 \\
& y_{t}^{c s u}+y_{t}^{c s b} \leq 1 \quad \forall t \in \mathcal{T} \\
& y_{t}+y_{t}^{c s b} \leq 1 \quad \forall t \in \mathcal{T} \\
& y_{t}^{c s u p} \geq y_{t}^{c s u}-y_{t-1}^{c s u} \forall t \in \mathcal{T}: t \geq 2 \\
& y_{t}^{c h s p} \geq y_{t}-\left(1-y_{t-1}^{c s b}\right) \forall t \in \mathcal{T}: t \geq 2 \\
& y_{t-1}^{c s d} \geq\left(y_{t-1}-y_{t}\right)+\left(y_{t-1}^{c s b}-y_{t}^{c s b}\right) \forall t \in \mathcal{T}: t \geq 2
\end{aligned}
$$

Constraint (3.26a) tracks start-up energy inventory, and Constraint (3.26b) allows nonzero inventory only during periods of cycle start-up. Constraint (3.26c) allows normal cycle operation only when start-up has been completed, when the cycle was previously operating, or when the cycle has been in standby mode. Constraint (3.26d) limits the cycle start-up rate, and Constraint (3.26e) enforces a maximum thermal power consumption limit by the power cycle. When operating, the cycle must produce a minimum amount of power enforced by Constraint (3.26f). Constraint (3.27a) determines electrical power production based on a linear cycle performance curve and the ambient temperature efficiency. The positive change in electrical power production is determined by Constraint (3.27b). The presence of $\dot{w}_{t}^{\delta}$ in the objective function provides a disincentive to vary power production from one time step to the next, thereby reducing system cycling and more closely representing operator-preferred generation profiles. The appropriate magnitude of this penalty parameter is unknown but is explored further in a sensitivity analysis provided 
in Section 4. Constraints (3.27c) and (3.27d) ensure that if the net power production upper limit is greater than or equal to that of the lower limit in any given time period, then that former production level must exceed that actually produced when efficiency is accounted for, less that from parasitics due to pumping power, heliostat field start-up, heliostat field tracking, power cycle standby, and tower piping heat trace. If the net power production upper limit is less than the lower limit in any given time period, the production level is zero. Start-up mode persistence is prevented in Constraint (3.28a). Standby mode can persist according to the analogous receiver requirements (Constraint (3.28b)). Standby and start-up modes cannot coincide (Constraint (3.28c)), nor can standby and power-producing mode (Constraint (3.28d)). Constraint (3.28e) enforces the penalty logic for start-up from an off state while (3.28f) enforces the penalty logic for start-up from a standby state. Constraint $(3.28 \mathrm{~g})$ enforces the logic for shut-down from a power-producing or standby state. Constraint (3.31b) ensures non-negativity for cycle start-up energy inventory, electrical power generation, and positive change in electricity production. Non-negativity for $x_{t}$ is ensured via Constraint (3.26f). Constraint (3.31d) enforces binary restrictions.

\subsubsection{Energy Balance}

The energetic state of the system implicates positive and negative power terms, and the charge state of thermal storage $\left(s_{t}\right)$ accounts for the cumulative difference between them. Several additional constraints regarding TES state of charge are enforced as follows:

$$
\begin{aligned}
& s_{t}- s_{t-1}=\Delta \cdot\left[x_{t}^{r}-\left(Q^{c} y_{t}^{c s u}+Q^{b} y_{t}^{c s b}+x_{t}+Q^{r s b} y_{t}^{r s b}\right)\right] \\
& \forall t \geq 2 \in \mathcal{T} \\
& x_{t+1}+ Q^{b} y_{t+1}^{c s b} \leq \frac{s_{t}}{\Delta_{t+1}^{r s}}-\mathbb{M} \cdot\left(-3+y_{t+1}^{r s u}+y_{t}+y_{t+1}+y_{t}^{c s b}+y_{t+1}^{c s b}\right) \\
& \quad \forall t \in \mathcal{T}: t \leq T-1
\end{aligned}
$$

Constraint (3.29a) ensures that energy into and out of TES balance with the charge state, and the conversion from power to energy introduces a time step parameter $\Delta$. Constraint (3.29b) addresses an artifact arising from the difference between the modeling time resolution (hourly) and the amount of time required to start the plant, which may not be in units of whole hours. If the power cycle is either running or in standby in time step $t$ and in time step $t+1$, and if the receiver starts up in time $t+1$, then the minimum charge level in TES in time $t+1$ must be sufficient to carry operation through the receiver start-up period. Note that $y_{t}+y_{t}^{c s b} \leq 1$ is enforced elsewhere. Equation (3.30) determines the expected fraction of each time step that would be used for receiver start-up, if applicable.

$$
\Delta_{t}^{r s}=\min \left\{1, \max \left\{\Delta^{\prime}, \frac{E^{c}}{\max \left\{\epsilon, Q_{t+1}^{i n} \Delta\right\}}\right\}\right\}
$$

Constraints (3.29a)-(3.29b) only track TES state of charge based on energy flow bookkeeping, not temperature.

Accounting for energy quality in the TES system via temperature of the molten salt introduces non-linear complexity and is not necessary in this formulation as previously discussed.

Variable bounds are enforced in (3.31a)-(3.31d), with (3.31b) bounding both the minimum and maximum amount of energy in storage.

$$
\begin{aligned}
& x_{t}^{r}, x_{t}^{r s u}, u_{t}^{r s u}, u_{t}^{c s u} \geq 0 \forall t \in \mathcal{T} \\
& x_{t}, \dot{w}_{t}^{\delta}, s_{t} \geq 0 ; s_{t} \leq E^{u} \forall t \in \mathcal{T} \\
& y_{t}^{r}, y_{t}^{r s u}, y_{t}^{r s u p}, y_{t}^{r s b}, y_{t}^{r h s p}, y_{t}^{r s d} \in\{0,1\} \forall t \in \mathcal{T} \\
& y_{t}, y_{t}^{c s u}, y_{t}^{c s b}, y_{t}^{c s u p}, y_{t}^{c h s p} \in\{0,1\} \forall t \in \mathcal{T}
\end{aligned}
$$

\subsubsection{Cycle Part-Load Correction}

An optimized dispatch profile may result in electricity production lower than the CSP plant design-point during certain time periods in order to conserve stored thermal energy for more favorable future market conditions, or to 


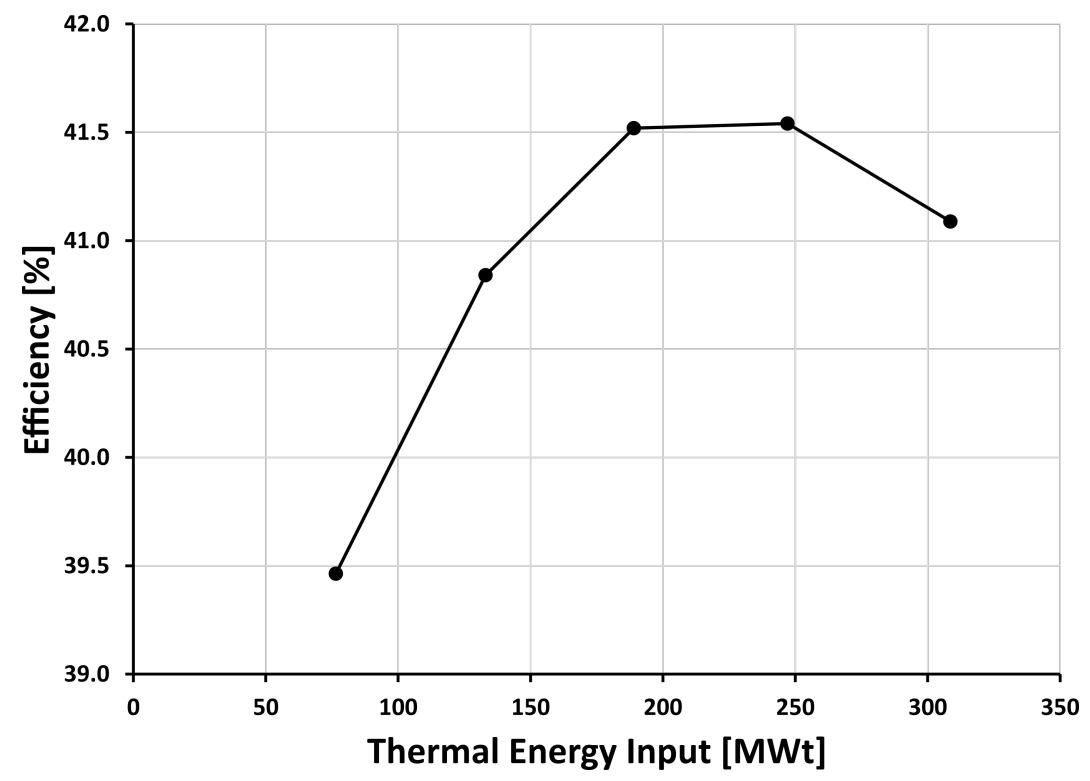

Figure 15. Cycle efficiency as a function of input thermal power represented using a piece-wise linear function.

avoid penalties associated with shut-down and start-up, for example. However, power cycle efficiency is adversely affected by departure from design, as shown in Figure 15 (Wagner).

The relationship between thermodynamic efficiency and thermal input is nonlinear and, consequently, poses computational challenges. In order to improve tractability in the corresponding optimization model, an approximately linear function of cycle thermal power consumption resolves the nonlinearity $\eta^{c y c l e}\left(x_{t}\right) \cdot x_{t}$ by modeling electrical output, shown in Constraint (3.27a). The linear coefficient is the quotient of the difference between the minimum and maximum output from the power cycle and the corresponding expression for the thermal power input.

$$
\eta^{p}=\frac{W^{u}-W^{\prime}}{Q^{u}-Q^{\prime}}
$$

\section{Dispatch Model Implementation}

The typical model instance contains 912 variables and 1,615 constraints. AMPL and CPLEX presolve reductions result in a problem with an average of 442 variables and 652 constraints, and an average run time on a Dell PowerEdge R410 server running Ubuntu 14.04 with 12GB RAM, 16 Intel processors at $2.72 \mathrm{GHz}$ each of 0.43 seconds per 48-hour horizon evaluation. By contrast, implementation of the model using LPSolve (Berkelaar, Eikland, and Notebaert), which is a freeware MIP solver platform for $\mathrm{C}++$, requires an average of 0.83 seconds per solve. Presolve reductions are less effective, producing instances with 890 variables and 920 constraints.

The number of time steps in the time horizon $(\mathcal{T})$ must be chosen with care, as it greatly affects the typical model described here as well as system techno-economic performance. The following considerations are relevant when choosing a time horizon duration: (i) the problem complexity grows exponentially with the time horizon length, and consequently, the amount of time needed for an annual simulation will also grow significantly; (ii) the optimized dispatch profile maximizes revenue within the allotted time horizon, and an insufficiently long horizon emphasizes near-term production at the expense of future, higher-value time periods; (iii) an optimal profile may require thermal energy to be held in storage overnight, and an insufficiently long time horizon (e.g., 24 hours) will fail to account for next-day requirements; and, (iv) given limitations on the number of branch-and-bound iterations and/or computation time per solve, an increased horizon length raises the likelihood of adopting a suboptimal dispatch profile, thereby negatively affecting expected plant performance. Figure 16 shows the impact of the time horizon length on the 
annual energy production and PPA price (discussed in the next section) for the reference plant defined in Table 15 below.

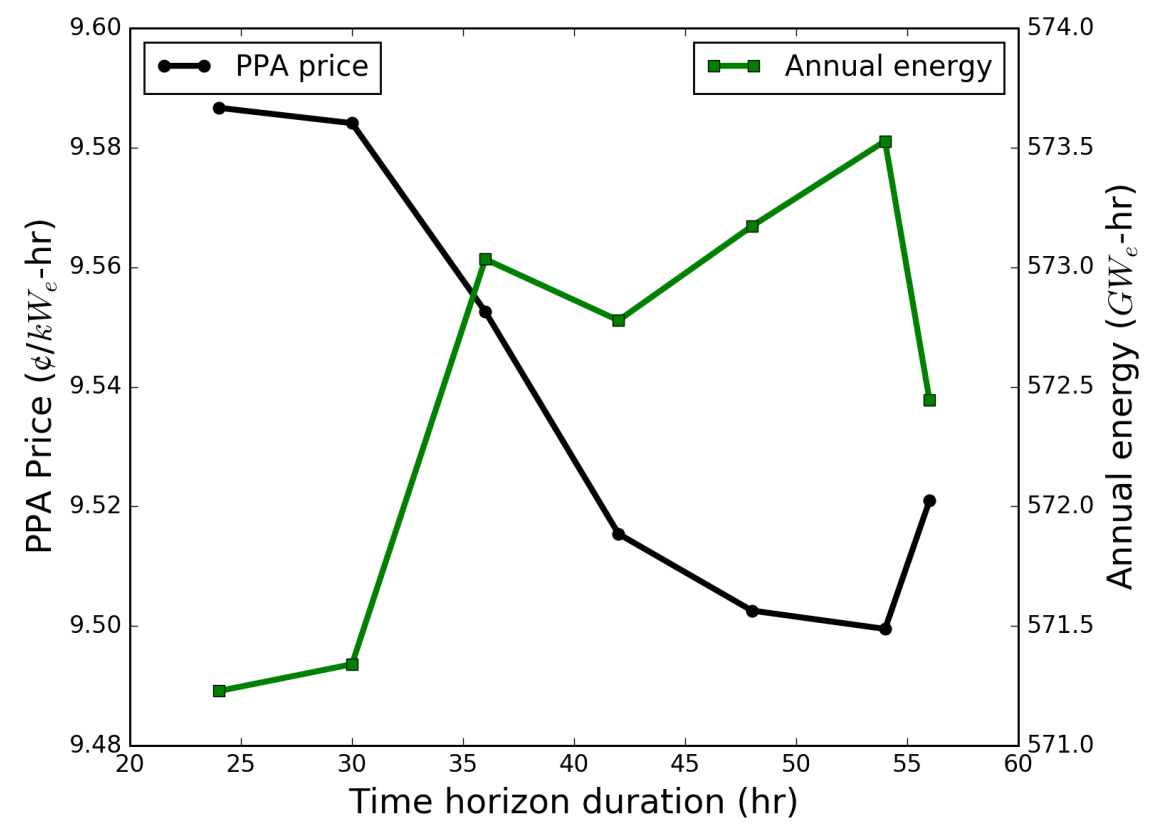

Figure 16. The impact of time horizon length (hours) on annual energy production and PPA price.

\subsubsection{Model Implementation}

Figure 17 illustrates the dispatch optimization model within SAM whose interface provides both input and output display. The user selects the technology and financial model, then modifies the inputs to emulate their technology configuration of interest, after which SAM simulates technical and financial performance by sending information from the interface to the SAM Simulation Core. Therein lies the molten salt power tower (SAM-MSPT) technology model that contains a solar field design algorithm called SolarPILOT and detailed calculators for determining weather data and the performance of the collector, receiver, power block, and TES subsystems.

The SAM-MSPT model simulates annual production by evaluating performance over a sequence of hourly time steps, at each of which the CSP controller determines the best operational mode given the conditions endogenous and exogenous to the system. The CSP solver ensures that all of the interconnected inputs and outputs among the calculators agree with respect to the thermodynamic state of the system. In summary, the architecture in Figure 17 characterizes a molten salt power tower plant with storage, in which the hour-by-hour plant operation protocol is determined using a 48-hour time horizon that rolls forward in 24-hour increments.

The Production Forecast Model determines expected future thermal energy generation of the solar field. While it is possible to implement a variety of techniques for predicting electricity pricing, ambient temperature, and direct normal irradiance, this approach uses "perfect forecasting" in which the model generates expected performance by reading ahead in the weather file. SAM-MSPT incorporates the time series data from the weather and pricing databases corresponding to the horizon over which the model is solved.

The heliostat field concentrates power on the receiver $\left(Q_{t}^{\text {helio }}\right)$ according to the instantaneous optical efficiency $\left(\eta_{t}^{s f}\right)$, direct normal irradiance $\left(d_{t}\right)$, and mirror area $\left(A^{s f}\right)$.

$$
Q_{t}^{\text {helio }}=\eta_{t}^{s f} d_{t} A^{s f}
$$




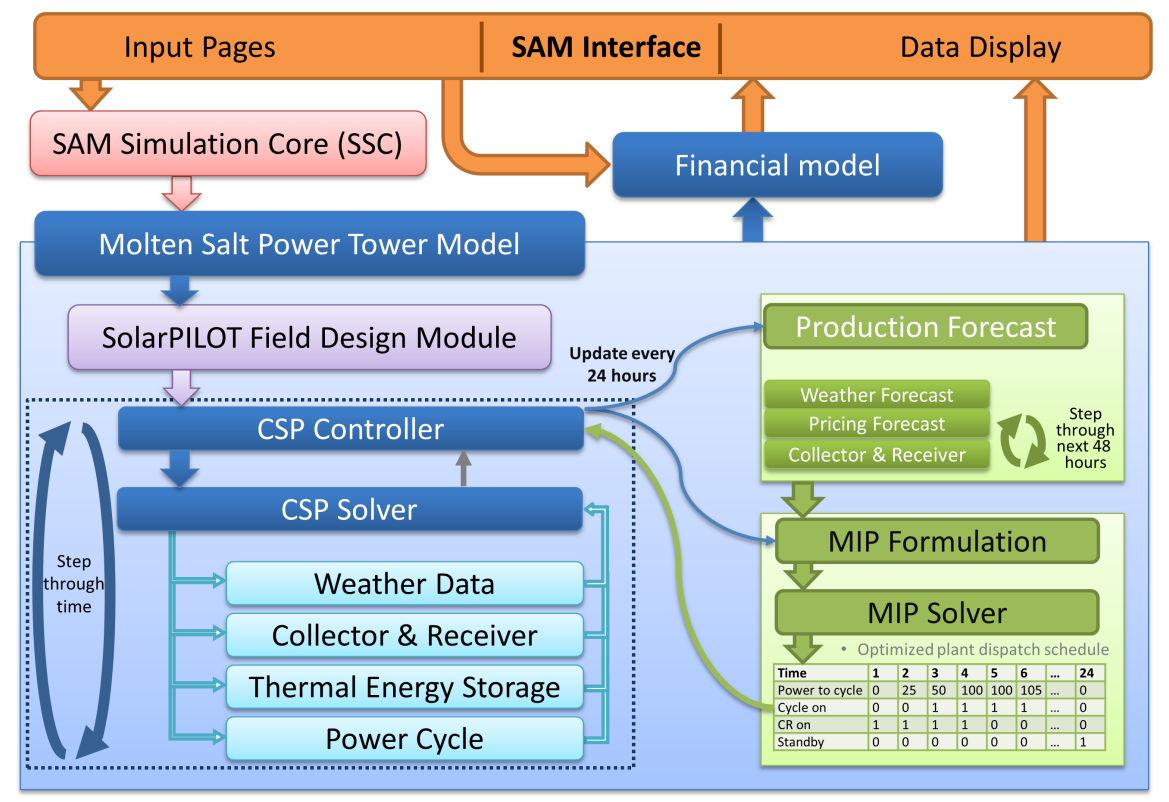

Figure 17. Information flow in the SAM-MSPT model. The MIP formulation is solved as a simultaneous set of equalities and inequalities, and the hourly solution profile is used by the CSP Controller to set target power production levels and operational states over the subsequent operational time horizon.

The total expected solar field production is the nonnegative difference of incident power on the receiver and convective $\left(Q_{t}^{c o n v}\right)$ and radiative $\left(Q_{t}^{\text {rad }}\right)$ thermal losses:

$$
Q_{t}^{i n}=\max \left[0, Q_{t}^{\text {helio }}-Q_{t}^{\text {conv }}-Q_{t}^{\text {rad }}\right] \forall t \in \mathcal{T}
$$

The following reduced-order relationships model the expected technical performance of the collector and receiver, providing a reasonably accurate approximation of expected field productivity. The collector field model generates a lookup table containing optical efficiency as a function of sun position, and the CSP controller supplies this information to the forecast model. The complexity of modeling the receiver thermal loss via convection and radiation from the heated surface necessitates a simplified forecasting model: an area-weighted average molten salt temperature is given as the weighted average of the inlet and outlet temperatures, where the coefficient is receiver-specific (Lata, Rodríguez, and Lara).

$$
T^{\text {eff }}=0.55 \cdot\left(T^{\text {out }}+T^{\text {in }}\right)
$$

Radiative losses are calculated at each time $t$ as:

$$
Q_{t}^{r a d}=A^{r e c} \sigma \epsilon\left(\left(T^{e f f}\right)^{4}-\left(T_{t}^{a m b}\right)^{4}\right)
$$

in which $A^{r e c}$ is the receiver surface area, $\sigma$ is the Stefan-Boltzmann constant, $\epsilon$ is the temperature-weighted surface emittance, and $T_{t}^{a m b}$ is the expected ambient dry-bulb temperature. Convective losses are expressed as a function of wind velocity for the molten salt technology, scaled by radiative loss. The coefficients in (3.37) are determined by regressing simulated data points that are generated using the SAM-MSPT detailed receiver model.

$$
\begin{aligned}
Q_{t}^{\text {conv }}= & \left(-5.645 \times 10^{-4} V_{t}^{3}+0.01561 V_{t}^{2}\right. \\
& \left.-0.00911 V_{t}+0.48124\right) Q_{t}^{r a d}
\end{aligned}
$$

where $V_{t}$ is the wind velocity at time $t$. 
The Engineering Performance Model (consisting of the CSP controller, CSP solver, and detailed performance calculators in Figure 17) predicts plant behavior and productivity over time using computationally expensive procedures derived from physically based, first-principles modeling of thermodynamics and heat transfer phenomena. The model's engineering performance behavior is validated and discussed in detail in (Wagner) and (Wagner and Gilman).

The MIP Mathematical Formulation, when solved with an appropriate algorithm, determines the performance and operation of the plant using the forecast model and various operational constraints (see Section 3.4.1.1).

The Pricing Model calculates the PPA price, which is the minimum value at which a power producer should agree to sell electricity in order to ensure that a specified internal rate of return is achieved. The PPA price is a useful surrogate for the profitability of a project in that it accounts for the variability in electricity value with time of day and time of year. As it is applied in SAM, the PPA price is multiplied by the hour-by-hour TOD or "tariff" rate to determine the value of electricity generated by the plant over time. SAM calculates the PPA price assuming a target internal rate of return (11\% in the current study) and an annual escalation rate of $1 \%$. For this reason - and somewhat counter-intuitively - a low PPA price is desirable. From the perspective of a power producer, a low PPA price improves its competitiveness. Alternatively, the PPA price could be specified and the internal rate of return maximized, and results from either approach would be equivalent. The results here translate the objective function value of $(\mathcal{R})$ into PPA price by taking fixed costs as sunk and maximizing revenue generated from electricity sales.

\subsubsection{Case Studies}

This study explores a range of plant TES sizes and solar multiples, the latter of which is defined as the ratio of solar field thermal power output to power cycle thermal input at design conditions. As the solar multiple increases, so too does the optimal amount of TES and the resulting plant capacity factor, but these values may be chosen independently. Table 15 provides a summary of key design parameters which are obtained from the default SAM-MSPT case. For this analysis, SAM automatically determines the heliostat field layout given the specified solar multiple and other design parameters. Each evaluation takes as fixed the TES and solar multiple and determines the optimal dispatch schedule for that system configuration.

In addition, this analysis considers four market scenarios (Figure 18), three of which have been adopted from Guédez et al., and one of which is the "generic summer peak" scenario used as the default for the SAM-MSPT model. The two-tier tariff market encourages daytime production with an evening spike. The pool price tariff introduces an additional morning spike and weights incentives seasonally. The fixed daytime tariff allows sales during daytime hours, but is unique in its binary nature; no revenue is available during nighttime operation. Finally, the SAM generic peak schedule combines features from the two-tier and pool price tariffs.

Using SAM-MSPT, this work compares the dispatch optimization methodology to the previous approach that relies on heuristic control which was configured to allow power generation any time the TES state of charge exceeded the threshold for minimum power cycle operation (satisfying Constraint (3.26f)). The cycle generates power at the design-point level unless insufficient energy is available in storage. Power cycle start-up occurs whenever energy in storage exceeds the quantity needed to deliver the start-up power for a single time period. The heuristic allows power generation until energy storage is exhausted each night, if applicable. This approach emphasizes maximum energy generation throughout the year.

\section{Results}

Table 16 presents the results of the PPA analysis, where the reported values correspond to the configuration with the minimum PPA price for the indicated scenario. Dispatch optimization successfully reduces PPA price compared to the heuristic dispatch method. Heavily weighted schedules (i.e., pool price and two-tier) lead to more substantial PPA price reductions (about 10-15\%), indicating that dispatch optimization is an essential aspect of plant operation for "peaker" markets that provide relatively short time windows of high-value energy pricing. The reader may also observe that systems operating in markets with more uniform tariff factors still benefit significantly from dispatch optimization, which alters the size of TES and the solar multiple at which PPA price is minimized. This implies 

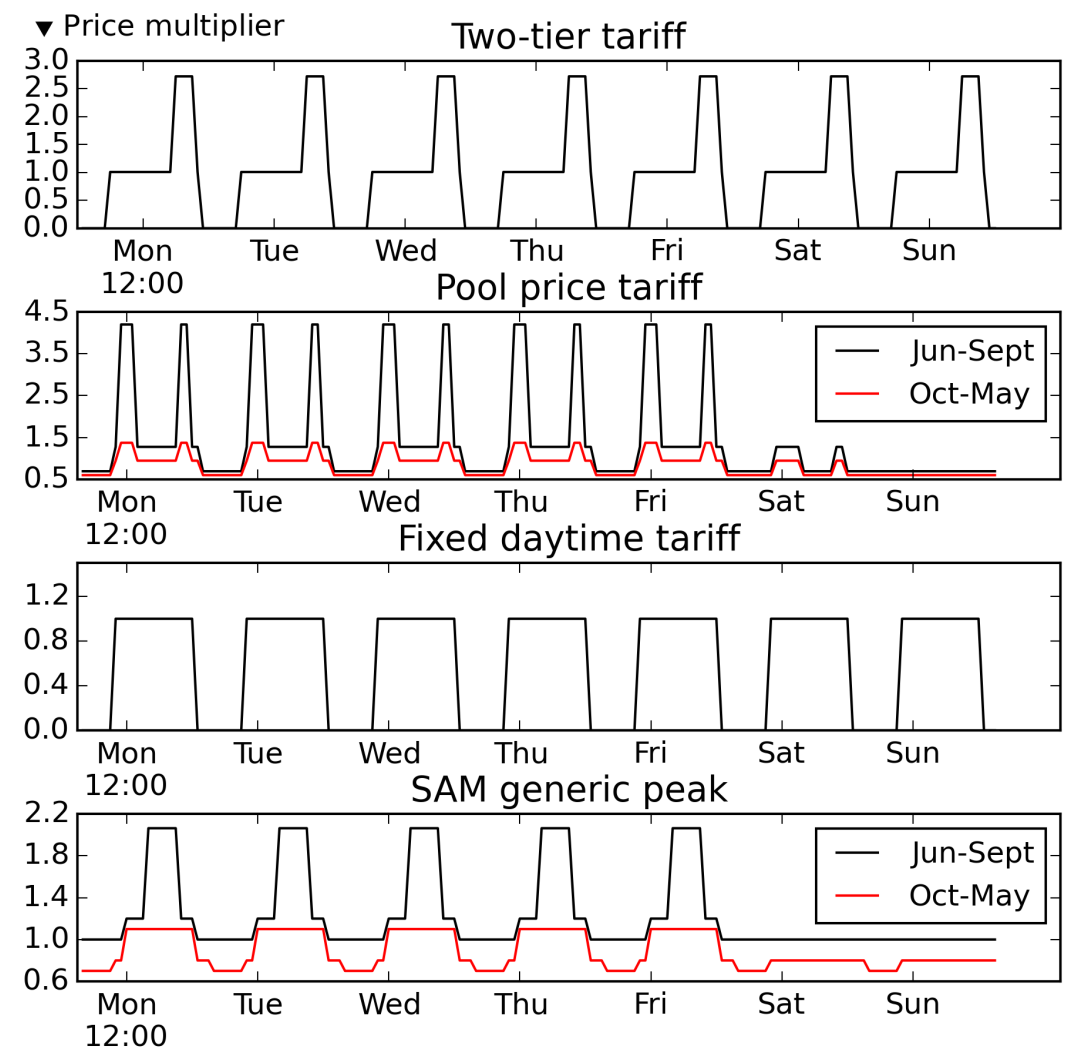

Figure 18. Market pricing scenarios presented by Guédez et al. These tariff schedules are implemented to determine the impact of dispatch optimization on system sizing.

that dispatch optimization should not be relegated to operational analyses, but rather should be part of the project screening and design process.

An important feature of dispatch optimization is the apparent improvement in the consistency of production during high-value time periods. Figure 19 illustrates this behavior for the pool price tariff scenario. Figures 3.19(a) and 3.19(b) show the hourly TES charge state profiles for each day of the year for both heuristic and optimized dispatch, respectively. Also shown on the plot are the tariff multiplier schedules for summer (red) and winter (blue) that determine the revenue associated with generation during a particular hour of the day.

Dispatch optimization substantially changes the daily operational profile. Whereas heuristic dispatch allows TES to discharge in the evening and late-night hours, optimized dispatch typically reserves some quantity of TES to allow morning start-up. The TES profiles show that heuristic dispatch is much more operationally repetitive relative to optimized dispatch, implying that the latter strategy accounts for expected resource availability and future pricing when scheduling TES usage.

Figures 3.19(c) and 3.19(d) show the distribution of electricity generation for each hour of the day over the course of the year. Each box-whisker plot describes the variability in electricity generation for each day at the specified hour, and the box limits indicate the extents of the first and third quartiles. The whiskers correspond to twice the inner quartile range. Points that lie outside of this limit are plotted individually and are distribution outliers.

Figure 3.19(c) shows that heuristic electricity production is highly variable in the early morning, evening, and nighttime hours (tall boxes) and is less variable during daytime hours (short boxes). The variability in electricity generation is inversely related to solar resource availability - an intuitive observation. However, production is highly 
variable during peak revenue hours as shown by the tariff multipliers. Figure 3.19(d) depicts optimized dispatch in which electricity generation variability is reduced during peak revenue hours.

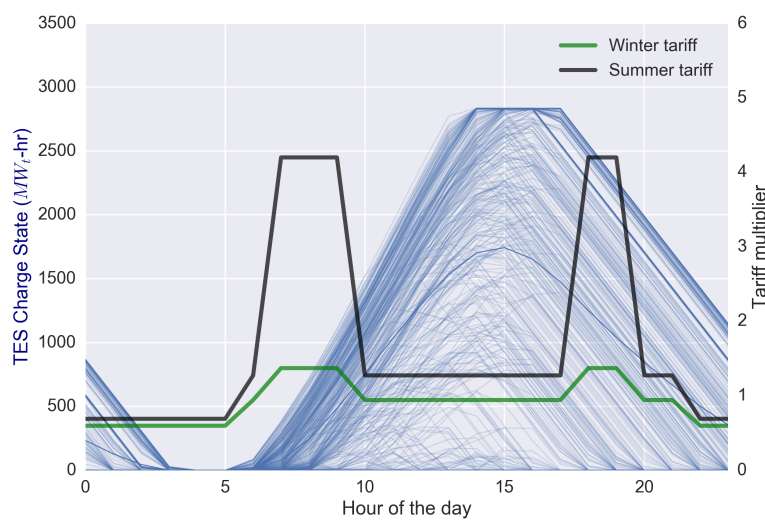

(a) Daily thermal storage charge state profile - heuristic dispatch

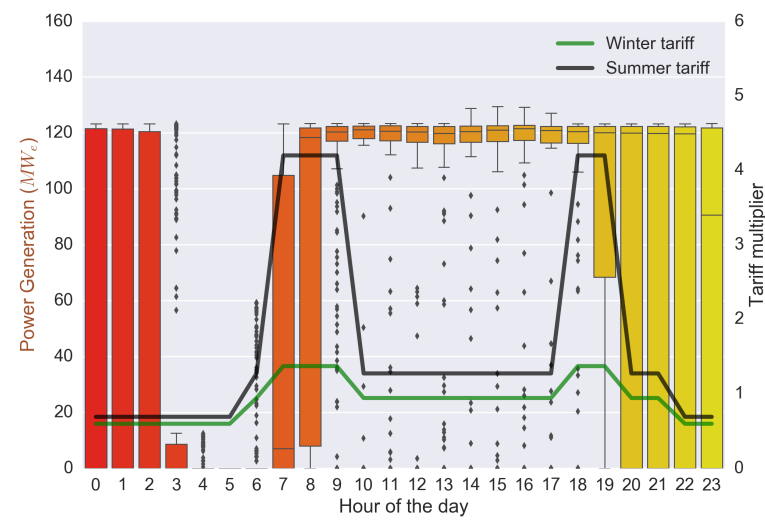

(c) Annual variability of electricity generation - heuristic dispatch

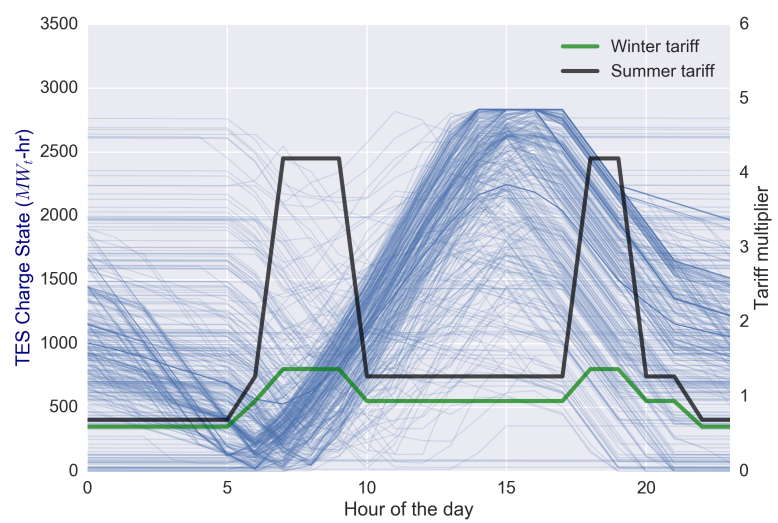

(b) Daily thermal storage charge state profile - optimized dispatch

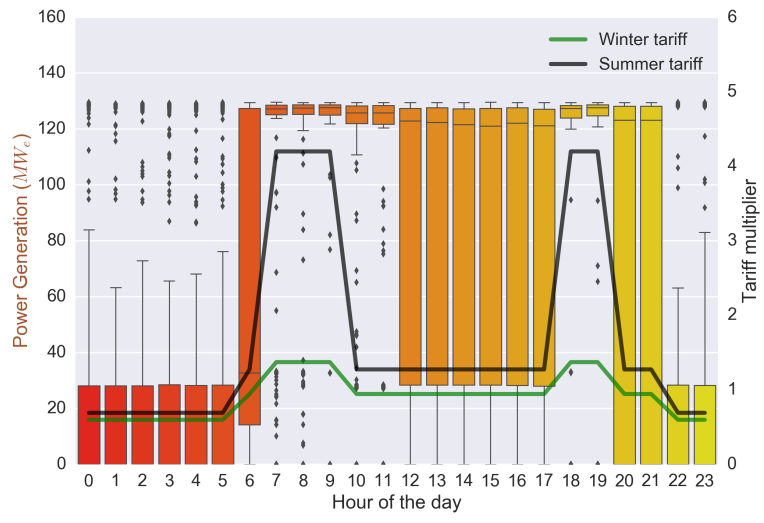

(d) Annual variability of electricity generation - optimized dispatch

Figure 19. Comparison of performance profiles for the pool price tariff schedule. Plots (a) and (b) show traces of the TES charge state for each day of the year. Plots (c) and (d) show box-whisker plots of daily electricity production variability over a year grouped by hour of the day. Each box indicates the mean annual electricity generation by hour, the first and third quartile limits (box limits), and two times the interquartile range (whiskers). "Outliers" are shown as blue dots. Summer (red) and winter (blue) tariff multipliers are overlaid on each plot.

\section{Penalty Parameter Sensitivity Study}

This study also seeks to understand the impact of operational cost parameters on both the generation profile and on PPA price. Plant operation that requires multiple daily start-ups or frequent production ramping may be difficult to execute and lead to additional maintenance costs over time. This work quantifies the revenue and production impact of obtaining "desirable" operational profiles (that is, relatively consistent output with few starts or ramp events).

The production change penalty, $C^{\delta \dot{W}}$, penalizes any positive change in power cycle electricity production from one hour to the next. Figure 20 presents the dispatch profile over several selected days in September in which four different penalty values are used. As the value of $C^{\delta \dot{W}}$ increases, the optimal solution sacrifices maximizing generation during periods of peak revenue and cycle efficiency to improve output stability. If increased to an extreme $\left(C^{\delta \dot{W}}=10.0\right)$, the dispatch profile approaches that of a baseload plant, only peaking for very short periods of time. 


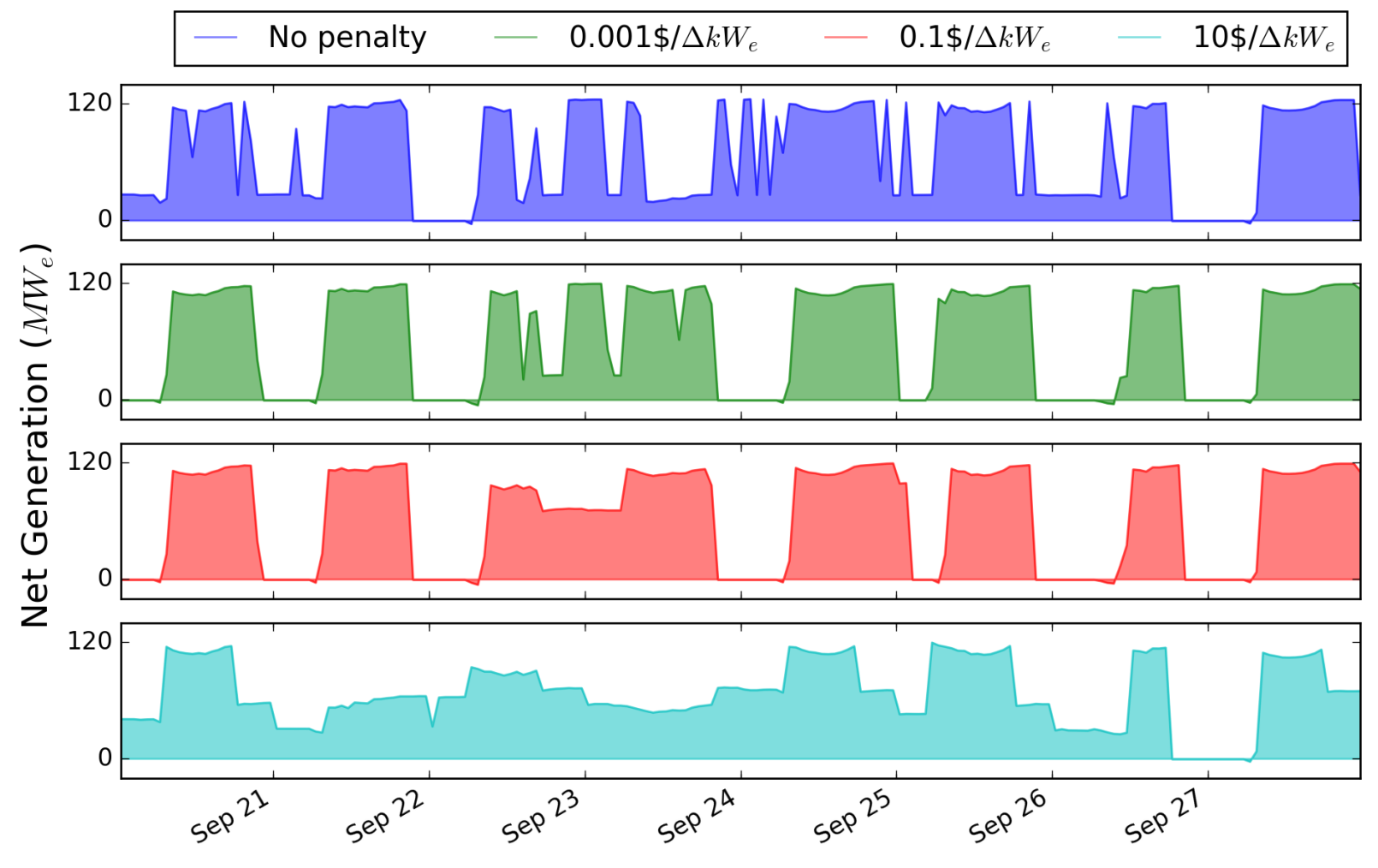

Figure 20. Plant power generation profile with varying change in production penalty values, $C^{\delta \dot{W}}$. (Penalties are given in the legend.)

This analysis leads to several considerations regarding the optimal design of a CSP plant given the potential ramping costs of the cycle. Namely, if production change costs truly are on the order of $10 \$ / \Delta k W e$, then the solar multiple, thermal storage, and power cycle sizes should generally be chosen to mimic a baseload plant. Penalties for frequent production changes would only be offset for the case in which TOD pricing variations are very large. Furthermore, an operator might reduce dispatch profile complexity but at some expense to apparent near-term plant profitability.

We also consider two TOD pricing scenarios ("Default" and "Peaker") in which the hours of TES and solar multiple are optimized for the no-penalty case. The first scenario is the generic summer peak profile in SAM, and the second is the pool price profile with spikes in pricing in the morning and evening and a price reduction during the middle of the day.

Figure 21 shows the impact of increasing the production change cost penalty on annual energy generation, PPA price, and number of turbine cycles per day, which is calculated as the total annual positive change in cycle production normalized to a value that corresponds to ramping the cycle from off to full load once a day for the entire year. If the number of cycles per day is greater than unity, then the turbine experiences more than one full cycle per day on average.

Several interesting observations arise from this analysis. First, the number of cycles per day decreases as the production change cost penalty increases, as intended. If production variability is not penalized at all, the optimal solution results in more than one cycle on average per day for both the Default and Peaker cases. As the penalty for changing production increases, the number of cycles drops significantly, which may be an important factor in increasing plant lifetime and reducing maintenance costs. Remarkably, the number of cycles can be reduced by $50 \%$ or more by manipulating this penalty without significant degradation of the objective function value. 
Second, the trends in reduction of cycles per day, annual energy generation, and PPA price are mirrored between the Default and Peaker cases. Both show that production change cost in the range of 0.5-2.0 $\$ / k W e$ minimizes PPA price and number of cycles per day, though the true costs of turbine ramping are not known and thus omitted from the PPA calculation. Non-coincidence of this outcome may imply an important cost threshold regarding CSP plants in general. Lastly, increasing the penalty leads to reduced annual output and increased PPA price below a threshold corresponding to $0.4-0.6$ cycles per day.

A final study considers the cycle start-up cost penalty's impact on the same performance metrics. This penalty is related to ramping cost, but differs in that it represents a penalty incurred for a discrete event that occurs only when the power cycle transitions from an off state to an on state, after which this penalty does not influence operation. Figure 22 shows the result of varying start-up cost for the Default case.

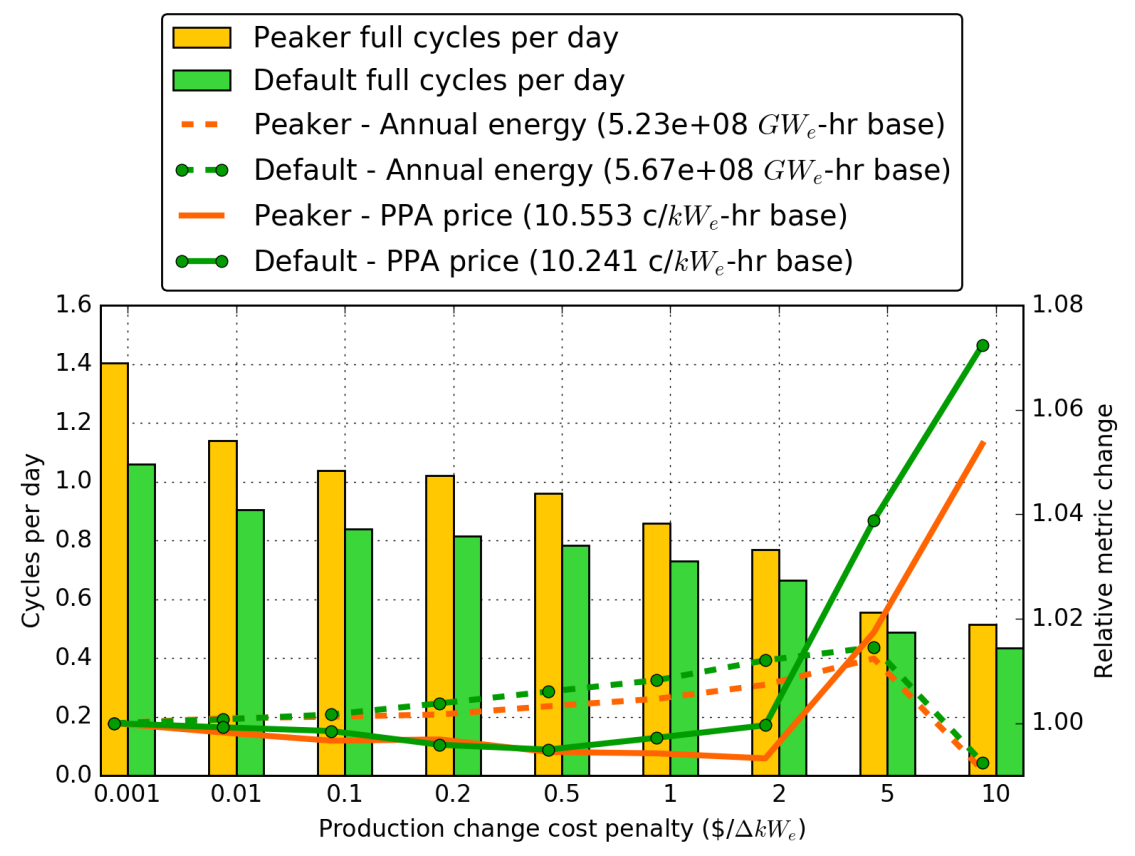

Figure 21. Impact of production change cost penalty on number of turbine cycles per day, annual energy generation, and PPA price for two pricing scenarios - a generic summer afternoon peak schedule (Default) and a morning/evening double-peak (Peaker) schedule. Annual energy and PPA price are shown as fractional values relative to the lowest-penalty case.

As with the production change penalty, the start-up penalty can significantly affect the behavior of the power cycle. A small cost of $\$ 100$ per start leads to a relatively large annual number of cycle starts (about 250). As a point of comparison, the number of cycle starts incurred using heuristic dispatch is 370 per year. The number of starts remains fairly constant (within variance that is to be expected based on numerical error in the solution) until the cost increases by a factor of 100 , and a factor increase of 1,000 reduces starts by approximately $50 \%$ without a significant effect on annual energy output and PPA price. Therefore, operational protocols that seek to minimize full cycle starts and stops can theoretically offer equally viable financial performance compared to more traditional approaches.

\section{Applications}

Results heretofore shown are readily applicable in practice, both for modeling and plant operations applications. First, modeling activities are fundamental to research, project development, and policymaking decisions, and an accurate estimate of technology performance directly impacts each of these areas. Researchers require tools that quantify the impact of advances in the technology, and utilize models to identify research priorities. The provision of 


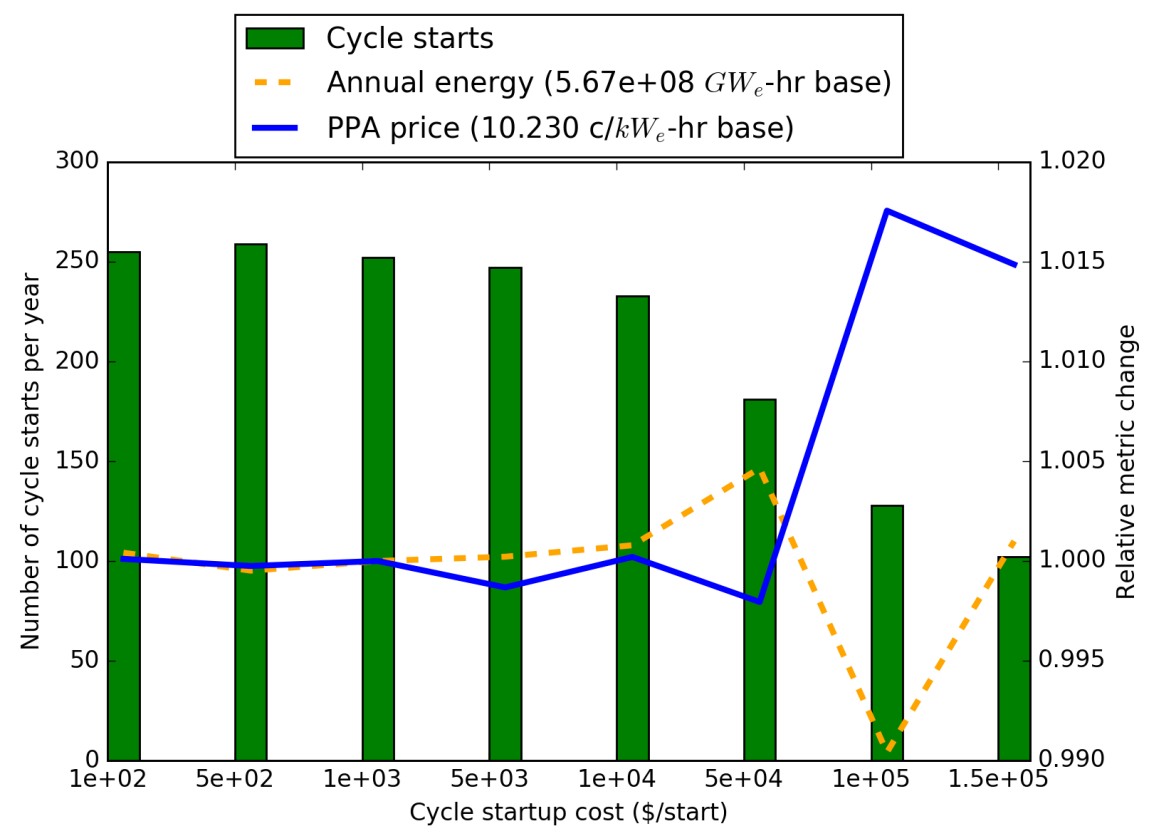

Figure 22. Number of cycle starts per year, annual energy output, and PPA price for the Default case with varying scenarios for cycle start-up cost.

a dispatch optimization tool in a publicly available software package enables assessment of the value of new thermal energy storage technologies - e.g., (Singh et al.), or power cycles - e.g., (Neises and Turchi), that otherwise may not interact with other subsystems as anticipated upon deployment. Project developers rely on models for initial plant design, attaining financing, project permitting, and finally, during plant operation. Dispatch optimization tools such as this are valuable for these purposes, and the authors present additional applied results and model validation in (Wagner et al.).

\subsubsection{Conclusions}

This section develops and implements a mixed-integer programming model within SAM to optimize the TES dispatch schedule for a molten salt power tower plant; this schedule provides a target power generation profile that is used in conjunction with a simulation model that evaluates plant performance at an hourly level over a year-long time horizon. SAM's detailed performance model mitigates some of the approximations present in the MIP formulation.

The results indicate that dispatch optimization can significantly improve plant revenue, though the gains vary with plant capacity factor and electricity markets; scenarios with heavily weighted pricing schemes or narrow windows of high revenue benefit the most, e.g., PPA price - indicative of the profitability of the plant - in these cases can improve by $10-15 \%$. Plant revenue is negatively affected by the energetic and financial cost of starting the solar receiver and power cycle equipment, and we show that optimized generation profiles can achieve a reduction in the number of turbine starts per year of $50 \%$ or more - in some circumstances - with little impact on project financial performance.

In summary, this model provides a methodology to optimize the trade-offs between component and subsystem performance, the effects of demand, and the amount of revenue obtained under various market schedules. Future work will incorporate, inter alia, forecast uncertainty, and more precise cost estimates of component operations and maintenance requirements. It will also examine how plant design and maintenance affect the overall cost and nature of the dispatch strategy. 


\subsubsection{Stochastic formulation}

The dispatch optimization model presented above relies on deterministic forecasts for DNI, price, and estimates of model parameters to calculate a single optimal operations profile. However, real-life forecasts are subject to uncertainty, and a purely deterministic dispatch model (with perfect information) overestimates the benefits of optimized dispatch while failing to provide a robust approach to undesirable operational cases.

Our approach for managing forecast uncertainty begins with developing a multi-stage stochastic model that is robust to a variety of likely scenarios. The model is derived from the deterministic program previously discussed, and is presented below. We assess the multi-stage program - which is computationally expensive - under contrived conditions to demonstrate the theoretical limits of the approach, and we compare performance to a heuristic model that we develop based on lessons-learned from the full model.

\subsubsection{Multi-stage stochastic program}

This section presents the mathematical formulation of the stochastic dispatch optimization model. Some parameters and all variables are indexed by time, $t$, and scenario, $\omega$, indicating their temporal and stochastic dependencies. Fundamentally, the model has a two-stage structure. Certain decisions are made in the first stage, and we must commit to these decisions prior to observing uncertain parameters including solar irradiance, energy prices, and temperature, which determines other model parameters. Other decision variables are in the second stage, and adapt to the stochastic realizations. Whether a decision variable belongs to the first stage, or second stage category in a particular time period depends on the nature of the variable. Below we specify six groups of variables. Some variables, such as the thermal power delivered by the receiver, are stochastic in all time periods, varying with realized DNI. Other variables governing receiver logic and start-up are first stage variables only in the very near term, and still other variables, such as the power cycle logic — as well as commitments of the receiver and power cycle, to be "on", i.e., in either standby or generation states—are committed to for longer periods in advance.

\subsection{Parameters and Sets}

Like the deterministic dispatch model, the stochastic model requires the initial operational state of the system, the collector field and receiver energy generation profile, the cycle conversion efficiency profile as a function of ambient temperature and thermal input, and the energy price or tariff profile; see Table 17.

Table 17. Parameters and sets.

\begin{tabular}{|c|c|c|}
\hline $\begin{array}{l}\text { Symbol } \\
\text { Sets }\end{array}$ & Units & Description \\
\hline $\mathcal{T}$ & & Set of all time periods in the time horizon, $T=|\mathcal{T}|$ \\
\hline $\mathcal{T}_{1}, \ldots, \mathcal{T}_{6}$ & & $\begin{array}{l}\text { Specifies number of time periods in the two-stage model's "first stage" for six groups of decision } \\
\text { variables; see Table } 18\end{array}$ \\
\hline$\Omega$ & & Set of all scenarios \\
\hline \multicolumn{3}{|c|}{ Time-indexed Parameters } \\
\hline$Q_{t, \omega}^{i n}$ & $\mathrm{~kW}_{t}$ & ${ }^{*}$ energy generated by the solar field in time $t$, scenario $\omega$ \\
\hline$P_{t, \omega}$ & $\$ / \mathrm{kW}_{e}-\mathrm{hr}$ & electricity sales price in time $t$, scenario $\omega$ \\
\hline$\eta_{t, \omega}^{a m b}$ & - & cycle efficiency adjustment factor in time $t$, scenario $\omega$ \\
\hline$\eta_{t, \omega}^{c}$ & - & normalized condenser parasitic loss in time $t$, scenario $\omega$ \\
\hline$\dot{W}_{t, \omega}^{\min }$ & $\mathrm{kW}_{e}$ & minimum net power production in time $t$, scenario $\omega$ \\
\hline$\Delta_{t, \omega}^{r s}$ & - & * estimated fraction at $t$ in $\omega$ used for receiver start-up \\
\hline$\dot{W}_{t}^{\text {net }}$ & $\mathrm{kW}_{e}$ & maximum net power production in time $t$ \\
\hline$\gamma_{t}$ & - & exponential time weighting factor; $\Gamma^{(t)}$, where $\Gamma \approx 0.99$ \\
\hline \multicolumn{3}{|c|}{ Time-invariant Parameters } \\
\hline$\phi_{\omega}$ & - & probability mass for scenario $\omega$ \\
\hline $\bar{P}_{\omega}$ & - & mean sales price under $\omega\left(\$ / \mathrm{kW}_{e}-\mathrm{hr}\right) ; \sum_{t \in \mathcal{T}} P_{t, \omega} / T$ \\
\hline$\eta^{d e s}$ & - & cycle nominal efficiency \\
\hline$\eta^{p}$ & - & * slope of linear approximation of power cycle performance curve \\
\hline$\tau$ & $\mathrm{hr}$ & frequency of optimization problem execution \\
\hline$E^{u}$ & $\mathrm{~kW}_{t}-\mathrm{hr}$ & energy storage capacity \\
\hline$E^{r}$ & $\mathrm{~kW}_{t}-\mathrm{hr}$ & required energy consumed to start receiver \\
\hline$E^{c}$ & $\mathrm{~kW}_{t}-\mathrm{hr}$ & required energy consumed to start cycle \\
\hline
\end{tabular}




\begin{tabular}{|c|c|c|}
\hline Symbol & Units & Description \\
\hline$E^{h s}$ & $\mathrm{~kW}_{t}-\mathrm{hr}$ & heliostat field startup or shutdown parasitic loss \\
\hline$Q^{u}$ & $\mathrm{~kW}_{t}$ & cycle thermal power capacity \\
\hline$Q^{\prime}$ & $\mathrm{kW}_{t}$ & minimum operational thermal power input to cycle \\
\hline$W^{u}$ & $\mathrm{~kW}_{e}$ & cycle electric power rated capacity \\
\hline$W^{\prime}$ & $\mathrm{kW}_{e}$ & minimum electric power output from cycle \\
\hline$\dot{W}^{h}$ & $\mathrm{~kW}_{e}$ & heliostat field tracking parasitic loss \\
\hline$\dot{W}^{b}$ & $\mathrm{~kW}_{e}$ & power cycle standby operation parasitic load \\
\hline$\dot{W}^{r s b}$ & $\mathrm{~kW}_{e}$ & tower piping heat trace parasitic loss \\
\hline$Q^{r u}$ & $\mathrm{~kW}_{t}$ & allowable power per period for receiver start-up \\
\hline$Q^{r l}$ & $\mathrm{~kW}_{t}$ & minimum operational thermal power delivered by receiver \\
\hline$Q^{r s d}$ & $\mathrm{~kW}_{t}$ & required thermal power for receiver shut-down \\
\hline$Q^{r s b}$ & $\mathrm{~kW}_{t}$ & required thermal power for receiver standby \\
\hline$Q^{c}$ & $\mathrm{~kW}_{t}$ & allowable power per period for cycle start-up \\
\hline$Q^{b}$ & $\mathrm{~kW}_{t}$ & standby thermal power consumption per period \\
\hline$L^{r}$ & $\mathrm{~kW}_{e} / \mathrm{kW}_{t}$ & receiver pumping power per unit power produced \\
\hline$L^{C}$ & $\mathrm{~kW}_{e} / \mathrm{kW}_{t}$ & cycle Heat Transfer Fluid (HTF) pumping power per unit energy consumed \\
\hline$C^{r s u}$ & $\$$ & penalty for receiver start-up (from 0 ) \\
\hline$C^{r h s}$ & $\$$ & penalty for receiver start-up (from hot standby) \\
\hline$C^{c s u}$ & $\$$ & penalty for cycle start-up (from 0 ) \\
\hline$C^{\text {chs }}$ & $\$$ & penalty for cycle start-up (from hot idle) \\
\hline$C^{\delta W}$ & $\$ / \mathrm{kW}_{e}$ & penalty for any positive change in electricity production \\
\hline$\Delta$ & $\mathrm{hr}$ & time step duration \\
\hline$\Delta^{\prime}$ & $\mathrm{hr}$ & minimum duration of receiver start-up in period \\
\hline$\alpha$ & $\$$ & conversion factor between unit-less and monetary values \\
\hline $\mathbb{M}$ & & a sufficiently large number \\
\hline
\end{tabular}

\subsection{Decision variables}

The decision variables in Table 18 describe energy states (thermal $k W_{t}$-hr or electric $k W_{e}$-hr), and power flows (thermal $k W_{t}$ or electric $k W_{e}$ ) in the system. Continuous variables " $x$," " $\dot{w}$," " $u$," and " $s$ " represent power and energy related to the receiver, power cycle, and thermal energy storage (TES). Binary variables " $y$ " enforce logic and operational modes. Parameters used to initialize decision variables at time $t=0$ follow the same notional format.

\subsection{Objective Function}

The deterministic dispatch model has been described in detail earlier. So, we do not revisit all of its logic. Rather, we simply point out differences in describing the objective function and constraints that arise with the stochastic model. In (3.38) we maximize expected profit.

$$
\begin{aligned}
& \text { maximize } \\
& \begin{array}{l}
\sum_{\omega \in \Omega} \sum_{t \in \mathcal{T}} \phi_{\omega}\left[\Delta \cdot P _ { t , \omega } \left(\gamma_{t}\left(1-\eta_{t, \omega}^{c}\right) \dot{w}_{t, \omega}-L^{r}\left(x_{t, \omega}^{r}+x_{t, \omega}^{r s u}+Q^{r l} y_{t, \omega}^{r s b}\right)-L^{c} x_{t, \omega}\right.\right. \\
\quad-\dot{W}^{h} y_{t, \omega}^{r}-\dot{W}^{b} y_{t, \omega}^{c s b}-\left(\dot{W}^{r s b}+E^{h s} / \Delta\right) y_{t, \omega}^{r s u} \\
\left.\quad-E^{h s} / \Delta \cdot y_{t, \omega}^{r s b}-E^{h s} / \Delta \cdot y_{t, \omega}^{r s d}\right) \\
-\gamma_{t}\left(C^{r s u} y_{t, \omega}^{r s u p}+C^{r h s} y_{t, \omega}^{r h s p}\right) \\
-\gamma_{t}\left(C^{c s u} y_{t, \omega}^{c s u p}+C^{c h s} y_{t, \omega}^{c h s p}+\alpha y_{t, \omega}^{c s d}+C^{\delta W^{\prime}} \dot{w}_{t, \omega}^{\delta}\right) \\
\left.+\gamma_{t}\left(\bar{P}_{\omega} \Delta x_{t, \omega}^{r}+\alpha y_{t, \omega}^{r}\right)\right]
\end{array}
\end{aligned}
$$

\subsection{Constraints}

The relationships among the variables and parameters are established with a set of simultaneous equations and inequalities. These constraints are presented below topically. 


\subsection{Receiver Operations}

Receiver operations constraints include:

Receiver Start-up

$$
\begin{aligned}
& u_{t, \omega}^{r s u} \leq u_{t-1, \omega}^{r s u}+\Delta \cdot x_{t, \omega}^{r s u} \forall \omega \in \Omega, t \in \mathcal{T}: t \geq 2 \\
& u_{t, \omega}^{r s u} \leq E^{r} y_{t, \omega}^{r s u} \forall \omega \in \Omega, t \in \mathcal{T} \\
& y_{t, \omega}^{r} \leq \frac{u_{t, \omega}^{r s u}}{E^{r}}+y_{t-1, \omega}^{r} \forall \omega \in \Omega, t \in \mathcal{T}: t \geq 2 \\
& y_{t, \omega}^{r s u}+y_{t-1, \omega}^{r} \leq 1 \quad \forall \omega \in \Omega, t \in \mathcal{T}: t \geq 2 \\
& x_{t, \omega}^{r s u} \leq Q^{r u} y_{t, \omega}^{r s u} \forall \omega \in \Omega, t \in \mathcal{T} \\
& \text { if } Q_{t, \omega}^{i n}=0 \text { then: } \\
& \quad y_{t, \omega}^{r s u}=0 \quad \forall \omega \in \Omega, t \in \mathcal{T}
\end{aligned}
$$

Receiver Supply and Demand

$$
\begin{aligned}
& x_{t, \omega}^{r}+x_{t, \omega}^{r s u}+Q^{r s d} y_{t, \omega}^{r s d} \leq Q_{t, \omega}^{i n} \quad \forall \omega \in \Omega, t \in \mathcal{T} \\
& x_{t, \omega}^{r} \leq Q_{t, \omega}^{i n} y_{t, \omega}^{r} \quad \forall \omega \in \Omega, t \in \mathcal{T} \\
& x_{t, \omega}^{r} \geq Q^{r l} y_{t, \omega}^{r} \forall \omega \in \Omega, t \in \mathcal{T} \\
& \text { if } Q_{t, \omega}^{i n}=0 \text { then: } \\
& \quad y_{t, \omega}^{r}=0 \quad \forall \omega \in \Omega, t \in \mathcal{T}
\end{aligned}
$$

Logic Governing Receiver Modes

$$
\begin{aligned}
& y_{t, \omega}^{r s u}+y_{t, \omega}^{r s b} \leq 1 \quad \forall \omega \in \Omega, t \in \mathcal{T} \\
& y_{t}^{r}+y_{t, \omega}^{r s b}=y_{t, \omega}^{r o n} \forall \omega \in \Omega, t \in \mathcal{T} \\
& y_{t, \omega}^{r s b} \leq y_{t-1}^{r}+y_{t-1, \omega}^{r s b} \quad \forall \omega \in \Omega, t \in \mathcal{T}: t \geq 2 \\
& y_{t, \omega}^{r s u p} \geq y_{t, \omega}^{r s u}-y_{t-1, \omega}^{r s u} \omega \in \Omega, \forall t \in \mathcal{T}: t \geq 2 \\
& y_{t, \omega}^{r s s p} \geq y_{t}^{r}-\left(1-y_{t-1, \omega}^{r s b}\right) \forall \omega \in \Omega, t \in \mathcal{T}: t \geq 2 \\
& y_{t-1, \omega}^{r s d} \geq\left(y_{t-1}^{r}-y_{t}^{r}\right)+\left(y_{t-1, \omega}^{r s b}-y_{t, \omega}^{r s b}\right) \forall \omega \in \Omega, t \in \mathcal{T}: t \geq 2
\end{aligned}
$$

Nearly all of these constraints replicate those of the deterministic model, except that we now track them for each scenario, $\omega$. This includes tracking scenario dependencies in the decision variables as well as scenario dependencies in energy delivered by the solar field on the right-hand side of constraints (3.39f), (3.40a), and (3.40b). Another important distinction concerns constraint (3.41b). In the deterministic model this is a " $\leq 1$ " constraint, but here in involves the new decision variable, $y_{t, w}^{r o n}$, which indicates whether the receiver will be "on", i.e., either in standby mode or generating thermal power.

\subsection{Power Cycle Operations}

Cycle Start-up

$$
\begin{aligned}
& u_{t, \omega}^{c s u} \leq u_{t-1, \omega}^{c s u}+\Delta \cdot Q^{c} y_{t, \omega}^{c s u} \forall \omega \in \Omega, t \in \mathcal{T}: t \geq 2 \\
& u_{t, \omega}^{c s u} \leq \mathbb{M} y_{t, \omega}^{c s u} \forall \omega \in \Omega, t \in \mathcal{T} \\
& y_{t, \omega} \leq \frac{u_{t, \omega}^{c s u}}{E^{c}}+y_{t-1, \omega}+y_{t-1, \omega}^{c s b} \quad \forall \omega \in \Omega, t \in \mathcal{T}: t \geq 2
\end{aligned}
$$




$$
\begin{aligned}
& x_{t, \omega}+Q^{c} y_{t, \omega}^{c s u} \leq Q^{u} y_{t, \omega} \quad \forall \omega \in \Omega, t \in \mathcal{T} \\
& x_{t, \omega} \leq Q^{u} y_{t, \omega} \quad \forall \omega \in \Omega, t \in \mathcal{T} \\
& x_{t, \omega} \geq Q^{\prime} y_{t, \omega} \quad \forall \omega \in \Omega, t \in \mathcal{T}
\end{aligned}
$$

Power Supply and Demand

$$
\begin{gathered}
\dot{w}_{t, \omega} \leq \frac{\eta_{t, \omega}^{a m b}}{\eta^{d e s}}\left(\eta^{p} x_{t, \omega}+y_{t, \omega}\left(W^{u}-\eta^{p} Q^{u}\right)\right) \forall \omega \in \Omega, t \in \mathcal{T} \\
\dot{w}_{t, \omega}^{\delta} \geq \dot{w}_{t, \omega}-\dot{w}_{t-1, \omega} \forall \omega \in \Omega, t \in \mathcal{T}: t \geq 2 \\
\text { If } \dot{W}_{t}^{\text {net }} \geq \dot{W}_{t, \omega}^{\text {min }} \text { then: } \\
\dot{W}_{t}^{\text {net }} \geq \dot{W}_{t, \omega}\left(1-\eta_{t, \omega}^{c}\right)-L^{r}\left(x_{t, \omega}^{r}+x_{t, \omega}^{r s u}\right) \\
\quad-x_{t, \omega} L^{c}-y_{t, \omega}^{r s u}\left(\frac{\dot{W}^{r s b}}{\Delta}+\frac{E^{h s}}{\Delta}\right) \\
\quad-\dot{W}^{h} y_{t, \omega}^{r}-y_{t, \omega}^{c s b} \dot{W}^{b} \forall \omega \in \Omega, t \in \mathcal{T}
\end{gathered}
$$

else:

$$
\dot{w}_{t, \omega}=0 \forall \omega \in \Omega, t \in \mathcal{T}
$$

Logic Governing Cycle Modes

$$
\begin{aligned}
& y_{t, \omega}^{c s u}+y_{t-1, \omega} \leq 1 \quad \forall \omega \in \Omega, t \in \mathcal{T}: t \geq 2 \\
& y_{t, \omega}^{c s b} \leq y_{t-1, \omega}+y_{t-1, \omega}^{c s b} \forall \omega \in \Omega, t \in \mathcal{T}: t \geq 2 \\
& y_{t, \omega}^{c s u}+y_{t, \omega}^{c s b} \leq 1 \quad \forall \omega \in \Omega, t \in \mathcal{T} \\
& y_{t, \omega}+y_{t, \omega}^{c s b}=y_{t, \omega}^{c o n} \forall \omega \in \Omega, t \in \mathcal{T} \\
& y_{t, \omega}^{c s u p} \geq y_{t, \omega}^{c s u}-y_{t-1, \omega}^{c s u} \forall \omega \in \Omega, t \in \mathcal{T}: t \geq 2 \\
& y_{t, \omega}^{c h s p} \geq y_{t, \omega}-\left(1-y_{t-1, \omega}^{c s b}\right) \forall \omega \in \Omega, t \in \mathcal{T}: t \geq 2 \\
& y_{t-1, \omega}^{c s d} \geq\left(y_{t-1, \omega}-y_{t, \omega}\right)+\left(y_{t-1, \omega}^{c s b}-y_{t, \omega}^{c s b}\right) \forall \omega \in \Omega, t \in \mathcal{T}: t \geq 2
\end{aligned}
$$

Stochastic parameter $\eta_{t, \omega}^{a m b}$ appears in constraint (3.43a) and $\dot{W}_{t, \omega}^{\min }$ appears in constraint (3.43c). In an analogous manner to how $y_{\omega, t}^{r o n}$ modified a constraint for receiver operations, in constraint $(3.44 \mathrm{~d}) y_{\omega, t}^{c o n}$ indicates whether the power cycle is either in standby or generating power. We must commit to the $y_{\omega, t}^{c o n}$ (and $y_{\omega, t}^{r o n}$ ) decisions for several time periods into the future, while the choice of "standby" or "generating power" can be delayed and adapt to stochastic realizations of DNI and other uncertain parameters.

\subsection{Energy Balance}

$$
\begin{aligned}
& s_{t, \omega}-s_{t-1, \omega}=\Delta \cdot\left[x_{t, \omega}^{r}-\left(Q^{c} y_{t, \omega}^{c s u}+Q^{b} y_{t, \omega}^{c s b}+x_{t, \omega}+Q^{r s b} y_{t, \omega}^{r s b}\right)\right] \\
& \forall \omega \in \Omega, t \geq 2 \in \mathcal{T} \\
& x_{t+1, \omega}+Q^{b} y_{t+1, \omega}^{c s b} \leq \frac{s_{t, \omega}}{\Delta_{t+1, \omega}^{r s}}-\mathbb{M} \cdot\left(-3+y_{t+1, \omega}^{r s u}+y_{t, \omega}+y_{t+1, \omega}+y_{t, \omega}^{c s b}+y_{t+1, \omega}^{c s b}\right) \\
& \forall \omega \in \Omega, t \in \mathcal{T}: t \leq T-1
\end{aligned}
$$


Equation (3.46) determines the expected fraction of each time step that would be used for receiver start-up, if applicable, and this parameter appears in constraint (3.45b):

$$
\Delta_{t, \omega}^{r s}=\min \left\{1, \max \left\{\Delta^{\prime}, \frac{E^{c}}{\max \left\{\epsilon, Q_{t+1, \omega}^{i n} \Delta\right\}}\right\}\right\} \text {. }
$$

\subsection{Nonanticipativity}

Nonanticipativity constraints specify the number of time periods in the first stage using sets $\mathcal{T}_{1}, \ldots, \mathcal{T}_{6}$ and the categorization of decision variables in Table 18. If we were to solve the stochastic dispatch model without these nonanticipativity constraints then the model would amount to a separate model for each scenario, $\omega$, and the system would have perfect information regarding the future for each scenario. The nonanticipativity constraints require that we must commit to certain decisions now, prior to realizing the values of the stochastic parameters.

If we commit to power cycle production decisions, say, 10 hours into the future then $\mathcal{T}_{1}=\{1,2, \ldots, 10\}$, and if the receiver logic only requires more immediate commitments over the next three hours then $\mathcal{T}_{2}=\{1,2,3\}$. Assuming that $\Omega=\{1,2, \ldots,|\Omega|\}$ then the nonanticipativity constraints for group 1 and group 2 variables are:

$$
\begin{aligned}
& x_{t, \omega}=x_{t, 1}, \quad \forall \omega \in \Omega, t \in \mathcal{T}_{1} \\
& \dot{w}_{t, \omega}=\dot{w}_{t, 1}, \quad \forall \omega \in \Omega, t \in \mathcal{T}_{1} \\
& \dot{w}_{t, \omega}^{\delta}=\dot{w}_{t, 1}^{\delta}, \quad \forall \omega \in \Omega, t \in \mathcal{T}_{1} \\
& x_{t, \omega}^{r s u}=x_{t, 1}^{r s u}, \quad \forall \omega \in \Omega, t \in \mathcal{T}_{2} \\
& u_{t, \omega}^{r s u}=u_{t, 1}^{r s u}, \quad \forall \omega \in \Omega, t \in \mathcal{T}_{2} \\
& y_{t, \omega}^{r}=y_{t, 1}^{r}, \quad \forall \omega \in \Omega, t \in \mathcal{T}_{2} \\
& y_{t, \omega}^{r s u}=y_{t, 1}^{r s u}, \quad \forall \omega \in \Omega, t \in \mathcal{T}_{2} \\
& y_{t, \omega}^{r s b}=y_{t, 1}^{r s b}, \quad \forall \omega \in \Omega, t \in \mathcal{T}_{2} \\
& y_{t, \omega}^{r s d}=y_{t, 1}^{r s d}, \quad \forall \omega \in \Omega, t \in \mathcal{T}_{2} \\
& y_{t, \omega}^{r s u p}=y_{t, 1}^{r s u p}, \quad \forall \omega \in \Omega, t \in \mathcal{T}_{2} \\
& y_{t, \omega}^{r h s p}=y_{t, 1}^{r h s p}, \quad \forall \omega \in \Omega, t \in \mathcal{T}_{2} .
\end{aligned}
$$

Nonanticipativity constraints for the remaining four groups of decision variables in Table 18 follow similarly, although we note that $\mathcal{T}_{4}=\emptyset$ because these decision variables are stochastic in all time periods.

\subsection{Simple Bounds}

$$
\begin{aligned}
& x_{t, \omega}^{r}, x_{t, \omega}^{r s u}, u_{t, \omega}^{r s u}, u_{t, \omega}^{c s u} \geq 0 \forall \omega \in \Omega, t \in \mathcal{T} \\
& x_{t, \omega}, \dot{w}_{t, \omega}^{\delta}, s_{t, \omega} \geq 0 ; s_{t, \omega} \leq E^{u} \forall \omega \in \Omega, t \in \mathcal{T} \\
& y_{t, \omega}^{r}, y_{t, \omega}^{r s u}, y_{t, \omega}^{r s u p}, y_{t, \omega}^{r s b}, y_{t, \omega}^{r h s p}, y_{t, \omega}^{r s d} \in\{0,1\} \quad \forall \omega \in \Omega, t \in \mathcal{T} \\
& y_{t, \omega}, y_{t, \omega}^{c s u}, y_{t, \omega}^{c s b}, y_{t, \omega}^{c s u p}, y_{t, \omega}^{c h s p} \in\{0,1\} \forall \omega \in \Omega, t \in \mathcal{T}
\end{aligned}
$$

\subsection{Assessment of the multi-stage model}

To create extreme scenarios that help reveal the structure of stochastic programming solutions, we designed a binary clear-sky experiment, in which, independently, each day has a 50-50 chance of having either zero DNI all day or having the clear-sky values of DNI all day. Here, we momentarily restrict attention to the situation in which the peak price for energy is much higher than the nominal price, and then we discuss extensions to handle the more general case. Motivated by the characteristics of solutions to our stochastic programs, we propose a simpler two-phase 
policy-based approach, which maximizes the following quantities in sequence: (i) the electricity generation during the peak-price hours of the current day and (ii) the inventory at the end of the current day.

Given the inventory in thermal energy storage (TES) at the beginning of the day, the experimental policy first maximizes the electricity generation during peak price hours on that day. Let the resulting total amount of peak-hour generation be $\dot{W}_{p}(\mathrm{MWh})$. In the second phase, the policy approach maximizes inventory at the end of the day, subject to a constraint requiring that we produce at least $(1-\epsilon) \dot{W}_{p}$ during the peak hours, where $\epsilon$ is a small number; we use $\epsilon=0.00001$ in our experiments. We commit to the dispatch decisions of the second-phase optimization problem, update the state of the system accordingly, and roll forward to the next day, recalling that the realized DNI could be either zero or clear sky. We opt to maximize the peak-hour production first because of the time value of the money, and the fact that in our simple experiments the peak price is identical each day. The second optimization model helps hedge against scenarios with zero DNI in the future. By doing so, we can still collect revenue during peak hours of a subsequent day with no DNI.

Naively implementing the proposed policy can result in excessive receiver and/or power cycle start-ups and shutdowns. We can resolve this issue by incorporating in the second optimization model the actual objective function (termed profit here) of the dispatch model, which includes revenue as well as the start-up costs. By doing so, the new objective function is a weighted combination of profit and terminal inventory at the end of the day. Putting a large weight on the inventory component of the objective function yields an optimization model that is equivalent to maximizing terminal inventory.

We present the results of the proposed policy, and compare it to alternative dispatch strategies, in two experimental settings: (i) the binary (0/1) clear-sky synthetic data described above, and (ii) historically observed DNI values. We create the synthetic dataset using clear-sky values for Hanford, California. We construct the second experimental setting from historical observed DNI values using ISIS data for Hanford, California for years 2010-2012.

For the numerical experiments we report here, we define two electricity price points (high and low), and we assume the price structure is deterministic and does not vary from day-to-day. In this report, we fix $\rho=20$ as the ratio of high price to low price to amplify the effects we are investigating. Let $\Delta^{h}$ be the number of peak price hours on any day, and $\Delta_{t}^{h}$ be an indicator function for peak price at hour $t$. We set $\Delta^{h}=3$ hours and $\Delta_{t}^{h}=1$ for $t=12,13,14$; otherwise, $\Delta_{t}^{h}=0$.

We emphasize that unlike experimental results described in earlier reports, here we are not employing SAM. Rather, all the accounting of revenue is done in the dispatch optimization model. This means that we have perfect information on DNI during the current day of operations. Integrating this work with SAM is an important next step.

We compare the performance of three models: (i) the deterministic expected value problem (EV); (ii) the stochastic program described in previous reports (SP); and, (iii) the policy-based approach that we have sketched here. Over the course of one year, we compare the revenue obtained by these three approaches. Table 19 indicates that policy approach outperforms both the stochastic program and expected value problem in terms of revenue on the synthetic dataset.

Using the synthetic data, Figure 23 shows the dispatch profiles for each method on a sequence of days in February. The first day is a clear-sky realization, the next three days have scenarios of zero DNI, and the final day is again a clear-sky realization. The blue curve indicates the thermal power delivered by the receiver to the system, and hence the lack of the blue curve on the middle three days shows the realizations of zero DNI. The green line indicates the inventory level in TES, while the red and light blue lines show thermal input and electricity output of the power cycle, respectively. The initial TES inventories differ on the first day because these are snapshots of the rollinghorizon scheme under each method. Note that the expected value problem (EV) fails to generate electricity during the high-price hours on February 13 and 14. The EV problem "believes" that it will have DNI of half the clear-sky value, and hence fails to save adequate inventory. For the same reason, the EV solution did not fully utilize peak price hours on February 12, i.e., it produced at the high price only for an hour although the high price lasts for three hours. In contrast, the stochastic program (SP) generated more during the peak price hours on February 12 and 13. Still, SP fails to produce electricity on February 14 . We only solve the SP for two-day horizons at a time, and 
so it fails to see far enough in the future to save adequate TES inventory. On the other hand, the policy approach achieves full peak-price production on all three days, February 12-14. While the policy approach ensures high price production today, it also invests in the future peak price production in order to protect the system against worst-case scenarios.

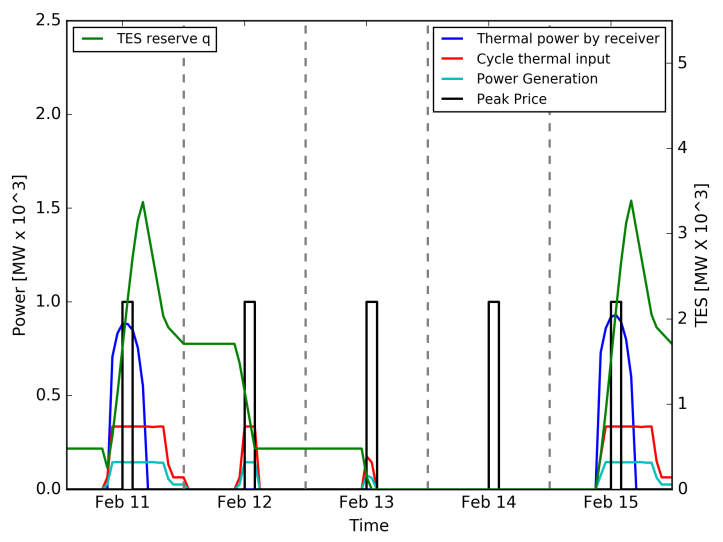

(a) Stochastic program

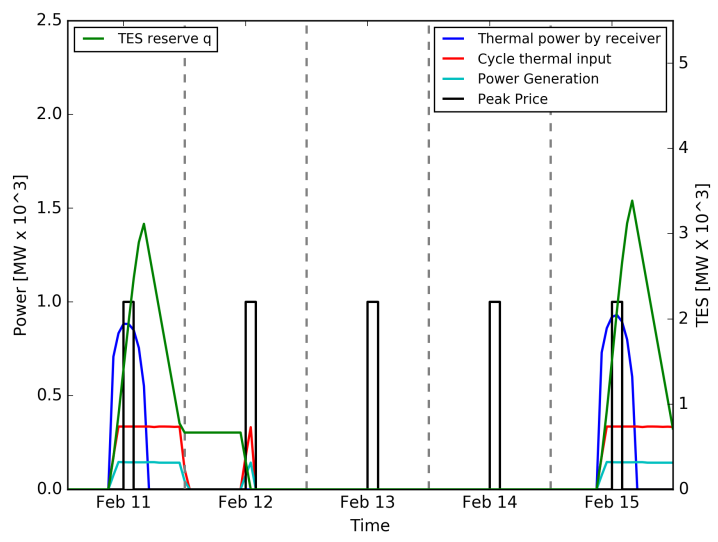

(b) Expected value problem

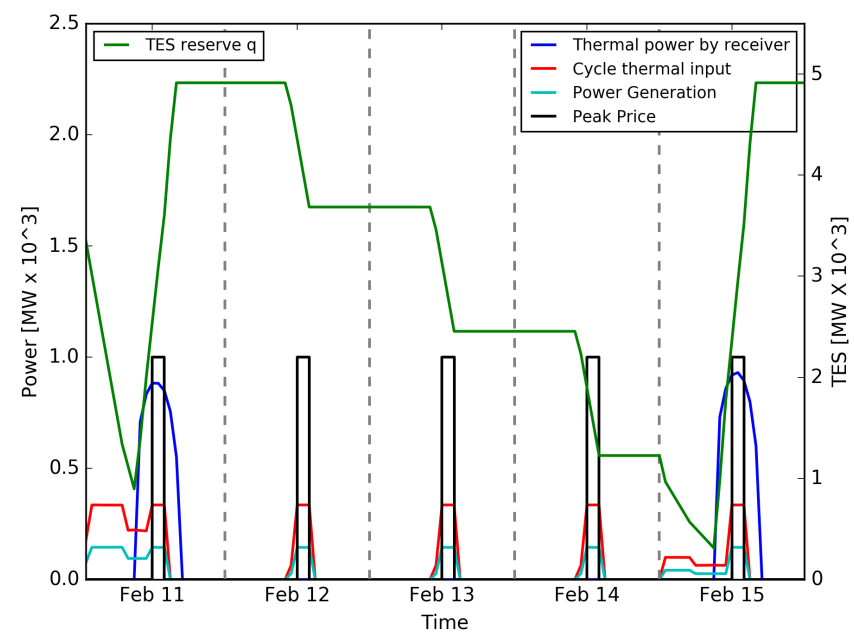

(c) Policy

Figure 23. Dispatch profiles over Feb 11-15 on the synthetic dataset

Table 20 provides numerical results for the 2010-2012 historical years for Hanford, which are analogous to those in Table 19 for the synthetic dataset. All three policies reflect the fact that the total DNI increased from 2010 to 2011 to 2012. Consistent with the intuition we have from Figure 23, it is not surprising that larger benefits from the stochastic program and the proposed policy are derived in years when DNI is scarcer.

One of the shortcomings of the deterministic model for optimizing dispatch is that large deviations from the point forecast result in lost revenue relative to strategies that hedge against uncertain DNI. The stochastic program for optimizing dispatch helps to overcome this issue, and its solutions are characterized by keeping larger amounts of inventory in TES, particularly when the peak-price value is large relative to the nominal price. Still, the limited time 
horizon (two days) of the stochastic program means that it, too, can be short-sighted. Our numerical results indicate that the simple policy we sketched in this report has the potential to mitigate this issue. While we presented results here for the case in which the peak-price value is large, the policy will need to be modified to reflect the ratio of peak-to-nominal prices, and also to handle uncertain energy prices. As a result, we are developing two approaches to guiding dispatch: (i) we will optimize the relative weight that we place on profit and terminal inventory in the policy we have described above, and (ii) we will use a stochastic dynamic program, which like the policy we describe here, can be solved quickly, and yet look further into the future to appropriately capture the benefit of holding TES inventory. Finally, as indicated above, an important next step will to be integrate this work with SAM.

\subsubsection{Heuristic model}

Stochastic programs can be computationally expensive to solve, especially when the number of scenarios is large. After studying the characteristics of solutions to the stochastic programs to optimize dispatch, we were motivated to explore computationally simpler approaches that produce dispatch decisions that mimic these characteristics. Results indicate that these solutions are typically at least as good those produced by the stochastic program itself, at least when peak energy prices are high and the pattern of peak and nominal prices are consistent from day to day. We describe our ideas here.

We now restate the dispatch problem using the following simplified expression:

$$
\begin{array}{cl}
\max _{x, s} & (1-\lambda) \cdot\left[\operatorname{Rev}_{1}\left(x_{1}\right)+\operatorname{Rev}_{2}\left(x_{2}\right)-\operatorname{Cost}_{1}\left(x_{1}\right)-\operatorname{Cost}_{2}\left(x_{2}\right)\right]+\lambda \cdot s_{2} \\
\text { s.t. } & \left(x_{1}, s_{1}\right) \in X_{1}\left(x_{0}, s_{0}\right) \\
& \left(x_{2}, s_{2}\right) \in X_{2}\left(x_{1}, s_{1}\right) .
\end{array}
$$

Here, the model is formulated for a 48-hour time horizon. The vector $x=\left(x_{1}, x_{2}\right)$ denotes operational decisions on day 1 and day 2, which include logical and continuous decisions concerning the power cycle and receiver. Variable $s_{1}$ denotes thermal energy storage at the end of day 1 . The objective function (3.49a) includes revenue terms and cost terms over the two days, and the constraints (3.49b) govern day 1 operations while constraints (3.49c) govern day 2 operations. The constraints on day 2 take the TES at the end of day $1, s_{1}$, as input, and some components of $x_{1}$ also inform permissible operations on day 2, e.g., through logical variables describing the operational state, ramping constraints, and more. The model is solved in a rolling-horizon manner, and hence day 1's constraints take into account previous day decisions, including TES via $\left(x_{0}, s_{0}\right)$.

The nominal dispatch optimization model uses $\lambda=0$. However, after exploring two-stage stochastic programming models, we found that model (3.49) performed well with $0<\lambda<1$. A modest reward, $\lambda>0$, on terminal inventory at the end of day 1 suffices to hedge against DNI and price uncertainty in day 2 . We formulated this simple variant of the nominal dispatch optimization model after studying the characteristics of solutions to two-stage stochastic programming models that we also formulated, but do not detail here. The approach adopted here also has the advantage of being computationally cheaper to implement than a full stochastic programming model. In what follows, we refer to this scheme as the "policy approach." As model (3.49) shows, the policy approach maximizes a weighted sum of: (i) the profit over a 48-hour period, and (ii) the inventory of thermal energy storage at the end of the first 24-hour period. The intuition for this type of policy is that the central tradeoff in operating a CSP plant under DNI and price uncertainty hinges on collecting profit today, possibly during medium- or low-price hours, versus saving energy to produce tomorrow when DNI may be low and/or prices may be high. In other words, following this policy can allow us to collect revenue during peak-price hours of a subsequent day.

Our dispatch optimization model recommends solutions to SAM, which attempts to follow energy production targets, while accounting for system thermodynamics with higher fidelity than our optimization model. Even though we solve a 48 -hour dispatch optimization model, only the solution for the first 24 hours is passed to SAM. Then, we step forward 24 hours in SAM and the process repeats. In what follows, we also analyze the benefit of having greater adaptivity in operations (i.e., not committing to decisions for the next 24 hours) and more recent information in our conditional DNI and price forecasts, by stepping forward, say, 12 hours rather than 24 in the rolling-horizon scheme. 
The approach with greater adaptivity, e.g., stepping forward only 12 hours, is appropriate when selling energy in the intra-day markets rather than the day-ahead market.

\subsubsection{Case study}

We report numerical results for (i) historically observed DNI values collected at Hanford, California using the SOLRAD data, and (ii) historical price data collected at a pricing node, named CONTADNA 1 N001, which is close to Hanford, CA both in DAM and RTM. For the RTM we use 15-min market, denoted by RTM15.

\section{Rolling-horizon scheme}

We follow a rolling-horizon scheme using the optimization model, the forecasting models, and SAM in order to mimic real-time application of the model. That is, we update the training dataset as DNI or price values are revealed, and then periodically refit the ARMA-X model to the revised training dataset. For this numerical study, we reestimate our model parameters every 10 days.

As sketched above, our rolling-horizon scheme can take different time increments, e.g., 12-hr, 8-hr, or 6-hr, since this allows us to use the most up-to-date DNI and price values in our forecasts and shorter-horizon forecasts tend to be more accurate. We produce varying lengths of forecasts for a given time horizon utilizing the most up-to-date information while rolling forward in specified time increments. For instance, given a 48-hr time horizon and 6-hr time increments, we generate 48,42,36,30-hr ahead forecasts for that 48-hr time window while including the most recent observations revealed in the last $6-\mathrm{hr}$ at each step. See Figure 24 for an illustration of this particular example. The rolling-horizon mechanism is mirrored in the dispatch optimization so that we only commit to those dispatch decisions in the specified time increment window, e.g., we implement the dispatch decisions in the first 6-hr window. The varying time increments can inform us about the value of operational adaptivity for a CSP plant.

We note that we can either perform our accounting for revenue within the simpler optimization model, or by embedding what we have just described within SAM. Of course, we want to do the higher-fidelity accounting using SAM because the former approach tends to be too optimistic. In what follows, we largely perform our accounting in SAM. That said, the optimistic approach can also be useful for deciding to not further pursue a particular option. We describe concrete examples below.

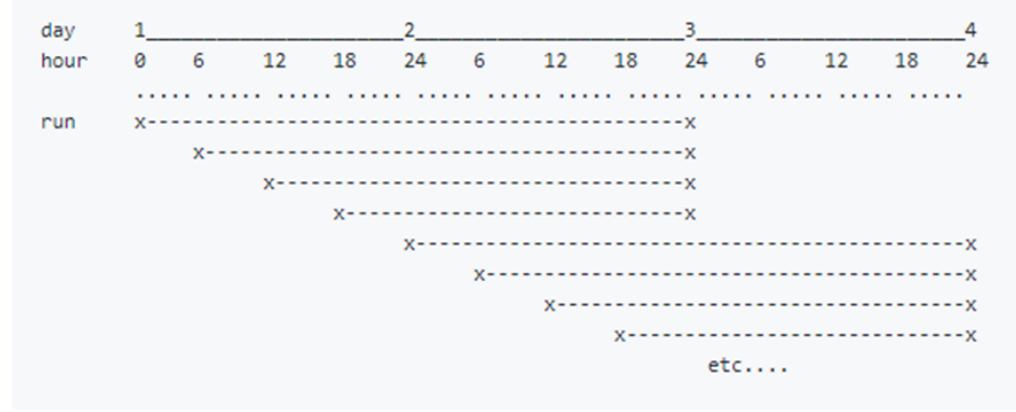

Figure 24. Illustration of 48-hr rolling horizon scheme with 6-hr time increments.

\section{Results}

We describe a number of distinct experiments in what follows. Some of these experiments use default SAM options for energy prices with pre-specified price multipliers based on time of day. In other experiments, we use historical energy prices in the DAM and RTM, coupled with forecasts of these prices. In addition, in some experiments we use the nominal dispatch model; i.e., model (3.49) with $\lambda=0$, and in other cases we use the policy-based dispatch model; i.e., model (3.49) with $\lambda>0$. These experiments are designed to isolate the cause, and quantify the potential benefit, of the various aspects of our proposed approach. 
For our first experiment, we assume a 48-hr time horizon with 24-hr time increments. We use the nominal dispatch model in which terminal inventory is not rewarded. We use default SAM settings for energy prices with time-of-day multipliers that repeat in an identical manner each day. We use the historically observed DNI values for Hanford, CA, for the three years 2010-2012 as our test dataset. We compare the performance of dispatch decisions obtained under three DNI input settings: (i) perfect information on future DNI, (ii) conditional DNI point forecasts obtained by equation (3.11), and (iii) the average of historical hour-by-hour DNI data. When computing this historical average we use the previous years' data in the identical week as well as the previous and subsequent week. In Table 3.21(a) we report the relative improvement in PPA price compared to dispatching under the previous years' DNI averages as a point forecast. For example, in 2010 the PPA price is $16.52 \%$ lower when using our conditional point forecast for DNI, relative to simply using the historical average. If we had perfect information on DNI when scheduling the plant, the PPA price instead is $21.09 \%$ lower in 2010 . Of course, we should not be able to improve upon a PPA price based on perfect information. We view these results as encouraging in that our conditional DNI forecasts allow us to capture the bulk of the upper bound on conceivable improvement over historical averages.

Table 3.21(b) illustrates the potential benefit of using the policy approach. Here, we use customized price multipliers in SAM with just two price levels, low and high. The ratio of high price to low price is denoted by $\rho$, and in these experiments we use $\rho=10$, a large value. We let $\Delta^{h}$ be the number of peak price hours on any day, and the experiments use $\Delta^{h}=3$ hours with high prices in hours $t=12,13,14$. Under perfect information, we obtain a PPA price, and suppose that value is 10 cents per $\mathrm{kW}$-hr. Then, the policy value of $9.87 \%$ in 2010 means that the PPA price under the policy base approach is 10.987 cents and that under the nominal dispatch model is 11.471 cents. In this way, a value of $0.00 \%$ is the ideal in Table 3.21(b) and larger values represent larger degradation in PPA over having perfect information. The policy and the nominal approach both already have the significant benefit afforded by the conditional DNI point forecast discussed above in Table 3.21(a).

Next we turn to incorporating price predictions in our experiments while using our best estimate of DNI, i.e., our conditional DNI point forecast. Analogous to our method with DNI, we form three price forecasts: (i) perfect information on future energy prices, (ii) conditional price forecast obtained by using the ARMA-X model, and (iii) the average of historical hour-by-hour prices data. The average is computed in an analogous way as for DNI, using three-week periods from previous years. We begin with DAM prices. We observe less than a $1 \%$ improvement in revenue using perfect information versus using historical averages. Here, this experiment and accounting is done purely within the optimization model rather than SAM. (Using SAM to compute PPA prices yields similar results.) Switching to RTM prices yields different results, suggesting a potential advantage. We attribute the distinction between the DAM results and the RTM results to the larger volatility of the latter prices. For these reasons, in what follows we restrict our attention to forecasting RTM15 prices to understand the value of conditional price point forecast. Since the historical RTM15 prices for our particular price node are available starting 2016, we present our results for year 2017, i.e., RTM15 prices for 2016 forms our training dataset. Table 22 shows the relative improvement in PPA price, calculated in SAM, over the baseline price method for a 48-hr rolling horizon with 24-hr time increments under the nominal dispatch model. As in results presented above, a $0.00 \%$ value is the ideal, albeit unachievable, resulting meaning no growth in PPA relative to having perfect information.

We next analyze the value of adaptability of the dispatch optimization model. If the CSP plant has the operational flexibility to be adaptive to changing weather conditions and electricity prices, it can make more informed dispatch decisions. The conditional DNI and price forecasts have improved accuracy over shorter horizons to better inform such decisions. We first return to understanding this in the context of uncertain DNI, using default energy prices in SAM. In Table 3.23(a), we show how PPA prices change with respect to the case of perfect information for 6- and 24-hr time increments.

Table 3.23(b) reports the results of an analogous experiment except that we use the conditional DNI forecast and the conditional price forecast at 6 - and 24 -hour increments.

\subsubsection{Hybrid systems dispatch}

In the last half decade, the solar industry has shown increasing interest in the design and deployment of CSP-PV hybrid systems. Preliminary analysis of CSP-PV hybrids demonstrate that the inclusion of photovoltaics can increase 
the capacity factor of a plant from $80 \%$ to roughly $90 \%$ in a cost-effective manner Green et al. The dispatchability of CSP systems with the use of thermal energy storage (TES) increases grid flexibility, which allows for greater penetrations of non-dispatchable renewable resources (i.e., solar PV and wind) Denholm. Solar hybridization can generate baseload power at a lower levelized cost of electricity than CSP or PV systems could alone Pan and Dinter.

Designing CSP-PV hybrid systems possesses its own unique challenges, requiring simulation to couple both subsystems, which has historically been done using monthly average hourly PV production. This method does not capture the variability of PV resource, thereby over predicting the production of the CSP-PV hybrid system. By integrating previously developed tools, the evaluation of annual performance of solar hybrid system designs is possible. The backbone of this analysis is the deterministic dispatch optimization model discussed previously. A visualization of the hybrid design and simulation tool is shown in Figure 25. At the top of the flowchart, a user sets high-level system characteristics according to which each plant subsystem is designed. Figure 26 provides a solar hybrid system layout.

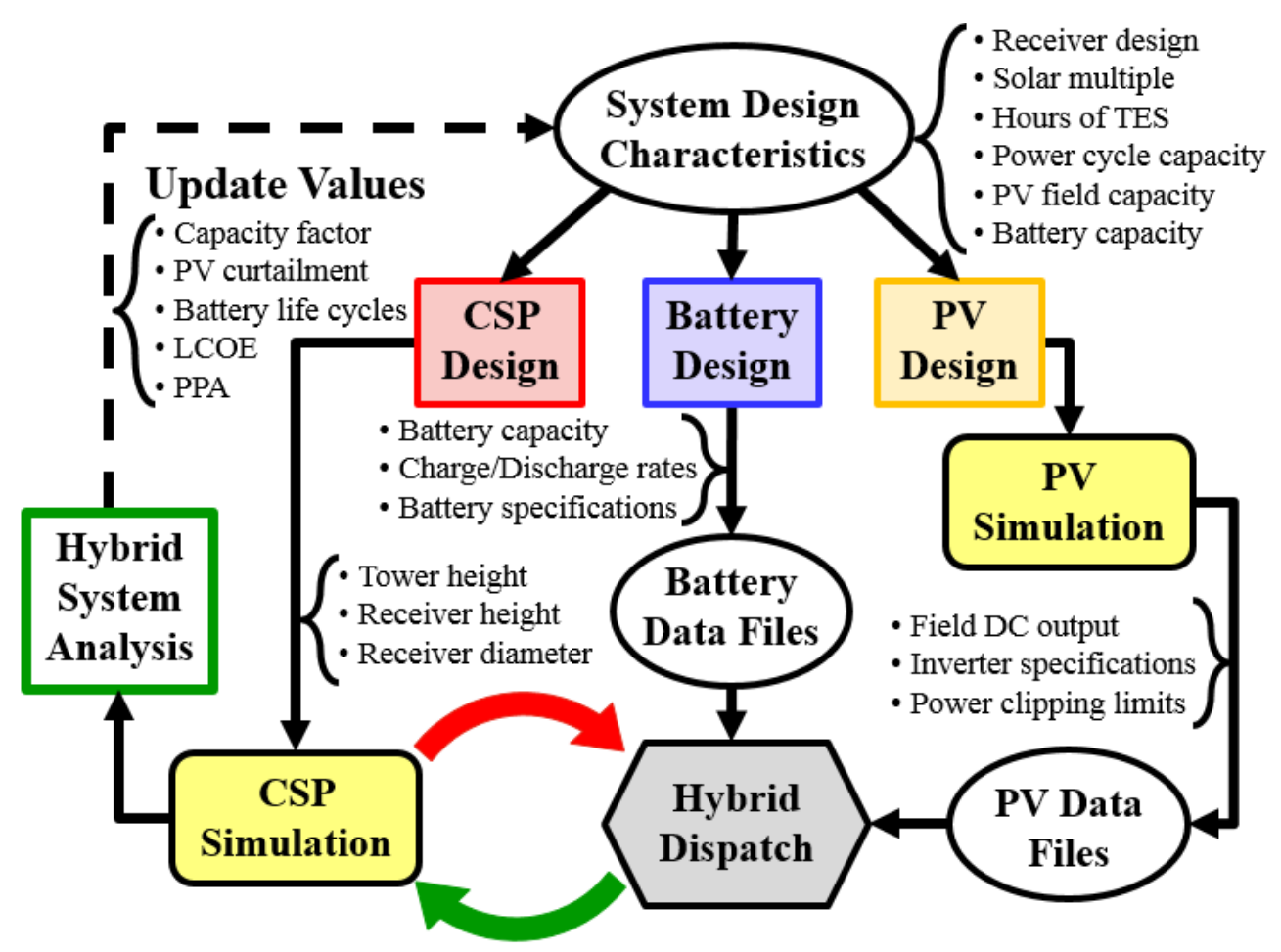

Figure 25. CSP-PV hybrid system design and simulation flow chart. CSP and PV simulations use SAM for performance prediction.

The PV field design is determined from the user input of a module (PV panel), a DC-to-AC inverter, and the PV field DC capacity. Then, SAM simulates the annual performance of the design and returns annual system metrics (i.e., annual energy, capacity factor, and inverter clipping losses). If the design does not meet user specifications, the field is redesigned and simulated. This process continues until the photovoltaic field performance meets user needs. When the photovoltaic field design is finalized, data for the hybrid dispatch model is generated. These data files contain inverter specifications and the predicted DC output for the field at each time step.

We additionally consider inclusion of a battery to enhance dispatch flexibility; for our study, we use a lithiumion battery. The battery system is connected to the PV. The battery reacts quickly to fast changes in net demand, delivering power to the grid. The battery's connection is represented by the green box on the left side of Figure 26. The battery can either be charged directly from the PV generation or through the rectifier connected to the AC 


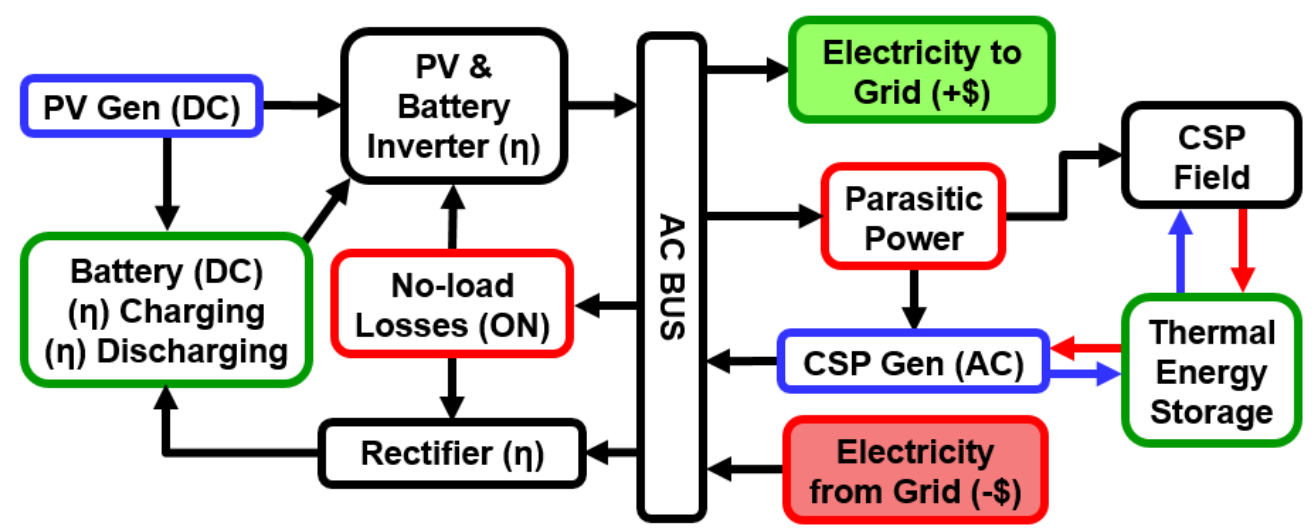

Figure 26. System layout consisting of CSP (with TES), PV field generation, and battery storage

(alternating current) bus. The battery discharges its energy through the inverter. Our model connects 3.4 Amp-hour lithium-ion cells to build the battery of the requisite capacity and voltage. The capacity of the battery is determined by the number of cells in parallel, while the voltage is determined by the number in series. This aspect of design may be considered by the optimizer, as it affects both capacity and maximum charge/discharge rate.

Once all the corresponding subsystems have a design, SAM's MSPT annual simulation begins, uses the rolling time horizon, and the hybrid dispatch optimization model to determine the subsystem operations that maximizes revenue. The hybrid dispatch optimization model was described in previous reporting.

\subsubsection{Hybrid dispatch optimization model}

To better represent operations of a CSP-PV hybrid system, we reformulate the dispatch model's objective function to include operating costs of the following plant sub-systems: receiver and CSP field, photovoltaic field, power cycle, and battery. Function (3.50) is the previously used objective, with different types of terms differentiated by color, and includes a solar curtailment bias term. Originally, we included this term to adjust CSP field curtailment to occur at the end of the day to be consistent with SAM simulations. However, we remove this term due to poor model behavior and replace its functionally with the receiver operating cost term, shown in Function (3.51), the new dispatch objective. The terms in teal represent power flow to and from the system in the form of electricity being sold or purchased. The terms in red represent fixed costs associated with receiver and cycle start-up. The terms in blue represent variable costs, given in $[\$ / \mathrm{kWh}]$, associated with sub-system operations.

$$
\begin{aligned}
& (\mathcal{R}) \text { Maximize } \sum_{t \in \mathcal{T}}[\Delta \cdot \overbrace{P_{t}}^{\text {Price }} \cdot \delta_{t}(\overbrace{\dot{w}_{t}^{S}}^{\begin{array}{c}
\text { Electricity } \\
\text { sold }
\end{array}}-\overbrace{\dot{w}_{t}^{P}}^{\begin{array}{c}
\text { Electricity } \\
\text { purchased }
\end{array}}) \\
& -\overbrace{C^{r s u} y_{t}^{r s u p}}^{\begin{array}{c}
\text { Receiver cold } \\
\text { start cost }
\end{array}}+\overbrace{C^{r h s} y_{t}^{r h s p}}^{\left.\begin{array}{c}
\text { Receiver hot } \\
\text { start cost }
\end{array}\right)}
\end{aligned}
$$

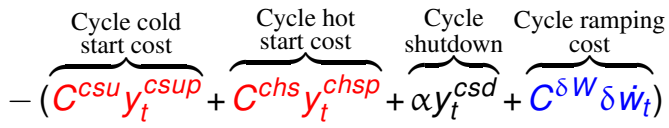

$$
\begin{aligned}
& \left.\left.+\delta_{t}\left(\bar{P} \Delta x_{t}^{r}+\alpha y_{t}^{r}\right)\right]\right\} \text { Solar curtailment bias }
\end{aligned}
$$




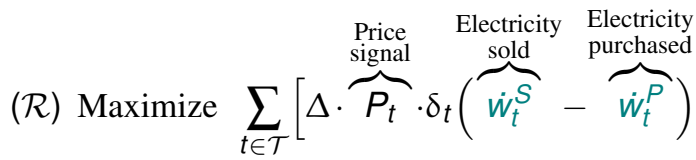

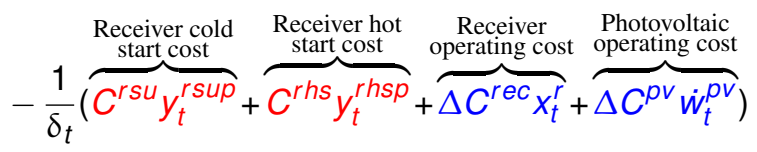

$$
\begin{aligned}
& -\frac{1}{\delta_{t}}(\overbrace{C^{c s u} y_{t}^{c s u p}}^{\begin{array}{c}
\text { Cycle cold } \\
\text { start cost }
\end{array}}+\overbrace{C^{c h s} y_{t}^{c h s p}}^{\begin{array}{c}
\text { Cycle hot } \\
\text { start cost }
\end{array}}+\overbrace{\alpha y_{t}^{c s d}}^{\text {Cycle }}+\overbrace{C^{\delta W_{\delta}} \delta \dot{W}_{t}}^{\text {shutdown }}) \\
& -\frac{1}{\delta_{t}} \overbrace{\Delta C^{\text {cycle } \dot{w}_{t}}}^{\begin{array}{c}
\text { Cycle } \\
\text { operating cost }
\end{array}}+\overbrace{\Delta C^{\text {bat }} \dot{w}_{t}^{b d}}^{\left.\begin{array}{c}
\text { Battery } \\
\text { operating cost }
\end{array}\right]}]
\end{aligned}
$$

Figure 27 contrasts dispatch profiles for which we use the same system design and constrain the electricity generation to be below a grid capacity of $100 \mathrm{MWe}$ (net). The only difference in the mathematical optimization models used to produce the two generation profiles is the objective function. The left axis in Figure 27 represents the dependent variables associated with gross electric power generation (positive) and storage (negative) from the individual plant sub-systems over the time horizon. This electric power generation figure is a slack area plot, meaning that sum of the areas at any given point in time is the total gross generation or consumption by the system. PV curtailment (in red) represents lost energy. Due to plant parasitic loads, gross power generation is greater than the grid capacity of $100 \mathrm{MWe}$. The right axis represents the following dependent variables: normalized thermal energy storage (TES) (red line), battery state of charge (SOC) (blue line), and PPA price multiplier (black line).

From Figure 27a, the generation profile using dispatch optimization with objective function (3.50) results in a majority of PV power being curtailed and the battery being cycled during the last five days of the time horizon. This behavior is thought to be due to only penalizing cycle ramping and the CSP solar curtailment bias term described earlier. However, this generation profile is sub-optimal because the operational costs of running the power cycle and CSP receiver are greater than that of PV generation. Therefore, it is intuitive from an economic standpoint that CSP receiver production is curtailed to a greater extent than PV production. Figure $27 \mathrm{~b}$ presents the generation profile resulting from having run the dispatch model with objective function (3.51), which includes operating costs of sub-systems, allowing for the dispatch model to evaluate the trade-offs between operations to ensure the most cost effective generation profile. There is still some PV curtailment during March $10^{\text {th }}$ and $11^{\text {th }}$, resulting from the power cycle minimum turn down and decision to avoid a cycle start cost penalty (by not turning off the cycle) for a short period of time during peak PV generation. Instead of shutting down the power cycle, the dispatch model captures excessive PV generation, to the extent possible, using battery storage, and curtails the remainder.

Table 24 presents annual simulation metrics for qualitative comparison of the dispatch model with objective functions (3.50) and (3.51). Objective (3.51) results in a substantial reduction in PV curtailment, from $24.9 \%$ to $0.3 \%$. There are also reductions in PPA price, the number of power cycle starts, battery life cycle usage, and average day solve time. In summary, hybrid system dispatch optimization with objective (3.51) provides a more efficient representation of CSP-PV hybrid system operational decisions.

Sub-system operating costs are not known with certainty. However, Table 25 presents a range of operating costs based on literature and engineering approximations. The battery operating cost does not include the cost of replacement due to capacity fade.

We improved the run times for hourly and 10-minute instances of the hybrid dispatch model by implementing variable elimination and passing a partial initial feasible solution. Variable elimination consists of removing variables from the model whose values are necessarily zero in any optimal - or even feasible - solution. For example, there 

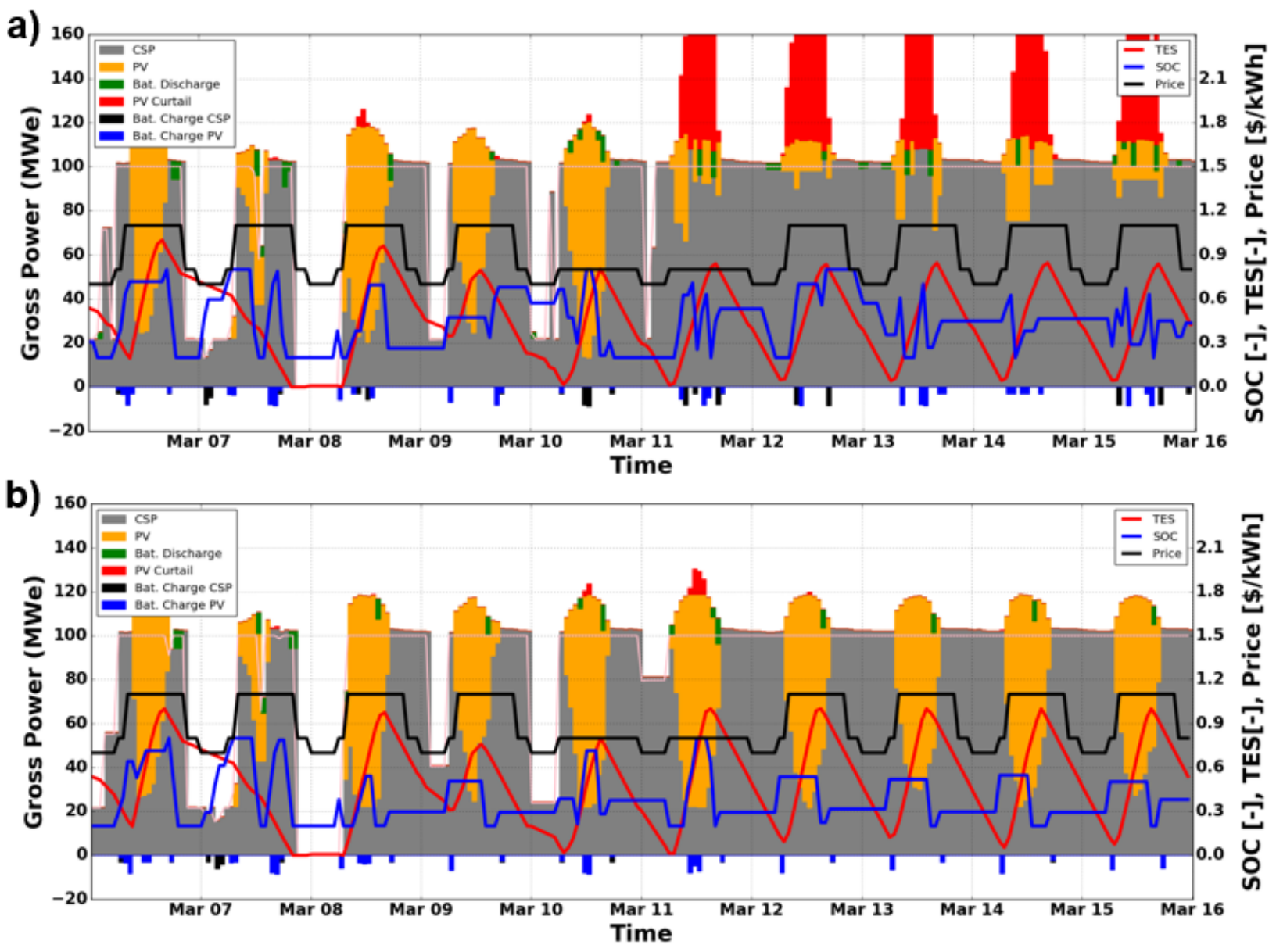

Figure 27. Example gross electric generation profiles using dispatch optimization (a) with objective function (3.50) and (b) with objective function (3.51). The left axis represents the dependent variables associated with gross electric power generation (positive) and storage (negative) from the individual plant sub-systems over the time horizon. The sub-systems include: CSP power cycle (gray), PV field (orange), battery discharge (green), PV field curtailment (red), battery charging by the power cycle or grid (black), and battery charging by the PV system (blue). PV curtailment (in red) represents lost energy. The right axis represents the following dependent variables: normalized thermal energy storage (red line), battery state of charge (SOC) (blue line), and PPA price multiplier (black line).

is a subset of time during which solar energy is available, which constitutes the solar day. We can therefore remove variables representing CSP thermal or PV field power generation outside of the solar day.

Our dispatch optimization model solves on a rolling 48-hour time horizon. Each time the model is solved, decisions spanning the first 24-hours are passed back to SAM for simulation. After simulating 24-hours, SAM reposes the 48-hour dispatch problem. To try to reduce solve times, we pass the last 24-hours of the 48-hour solution to the next 48-hour dispatch problem as a partial initial feasible solution. This process helps the solver start with a reasonable "guess" of the optimal dispatch for the first day in the 48-hour problem.

Future work for the hybrid dispatch model should include adjustments to model formulation to better reflect the current industry concerns, and the inclusion of formulation cuts, solver parameter tuning, and a heuristic to provide a whole initial feasible solution to further improve solve times. 


\subsubsection{Efficient subhourly dispatch optimization}

The hybridization of PV-CSP-battery technology is particularly interesting when considering short-duration variability (e.g., on the order of 5-15 minutes) in the solar resource. However, solving the model as previously stated using sub-hourly time steps proves to be computationally burdensome. In this section, we present techniques to reduce solution times for a sub-hourly dispatch model which is then able to capture solar resource variability with respect to plant operations. Specifically, we reformulate the dispatch problem to consist of two time horizons, each with different time fidelities: (i) sub-hourly fidelity for the first 24 hours that corresponds to that of the input weather data file (i.e., 10-minute); and (ii) hourly time fidelity for the remainder of the horizon, associated with aggregated SAM input data, i.e., fidelity that correspond to that used in the day-ahead energy market. The aggregated data in (ii) improves the tractability of the problem instances by reducing the number of variables in the second 24-hour time window.

A simple case has each of the two windows spanning a day, as shown in Figure 28, "Version A." Considering the second 24-hour window at hourly, rather than 10-minute, fidelity reduces the number of time intervals by $41.6 \%$. Other variants are possible, and can invoke a sliding time window implementation (Figure 28, "Version B."); in this case, however, there is a trade-off between problem complexity (i.e., solution time) and the number of solves required to obtain a day-one solution. We suspect that moving to five-minute time intervals (i.e., those present in real-time energy market decision-making) would require Version B; however, more testing is necessary to confirm this hypothesis.

Original: 10 -minute time intervals

\begin{tabular}{|c|c|c|} 
Day 1 & Day 2 & Tot. \\
\hline 144 Time intervals & 144 Time intervals
\end{tabular}

Version A: Day 1: 10-minute, Day 2: hourly

\begin{tabular}{|c|c|} 
Day 1 & Day 2 \\
\hline 144 Time intervals & 24 Time intervals
\end{tabular}

Version B: Day 1: rolling time horizon @ 5-minute for first 6 hours, Rest of horizon: hourly

\begin{tabular}{|c|c|c|c|c|c|}
\hline \multicolumn{4}{|c|}{ Day 1} & Day 2 & \\
\hline 72 steps & \multicolumn{3}{|c|}{18 Time intervals } & 24 Time intervals & 114 \\
\hline Fixed & 72 steps & \multicolumn{2}{|c|}{12 Time intervals } & 24 Time intervals & 108 \\
\hline Fix & & 72 steps & 6 steps & 24 Time intervals & 102 \\
\hline \multicolumn{3}{|c|}{ Fixed } & 72 steps & 24 Time intervals & 96 \\
\hline
\end{tabular}

Figure 28. The original formulation has sub-hourly time intervals throughout the time horizon. Version A possesses sub-hourly time intervals during the first day and aggregates data to hourly fidelity for the second day. Version B possesses sub-hourly time intervals for a time window less than a full day, and requires multiple solves to produce a day-one solution - in this example, four six-hour solves using five-minute time fidelity.

After testing both versions of the new formulation with 10-minute time intervals, the greater reduction in solution times occurs using Version A. The distribution of sub-hourly dispatch problem solution times for a year's worth of solves are presented in Figure 29 for the original (left) and Version A (right) formulations; instances are run on a Quad-Quad core CPU at $2.93 \mathrm{GHz}$ with $32 \mathrm{~GB}$ RAM and 1TB HDD hardware. For each instance, we set a time limit of 120 seconds; for the original implementation, 55 problem instances reached this limit, resulting in likely sub-optimal solutions, while none reached the limit using Version A. Additionally, Figure 29 shows that the solve time distribution of the original formulation is highly variable with a mode (excluding runs that reached the time limit) occurring between 10 and 12 seconds. By contrast, solution times associated with Version A are concentrated between 0 and 4 seconds, with a mode occurring between 0.5 and 0.75 seconds. The total time required to solve a complete year using the original formulation versus Version A is about 5 hours in contrast to 12 minutes, respectively. That is, Version A results in a $96 \%$ reduction in total solve time compared to the original formulation, while 
the solution quality produced by Version A is as good as or better than that produced by the original formulation. We note that these improvements, while implemented on the hybrid system, are also applicable to dispatch optimization of a CSP-only system.
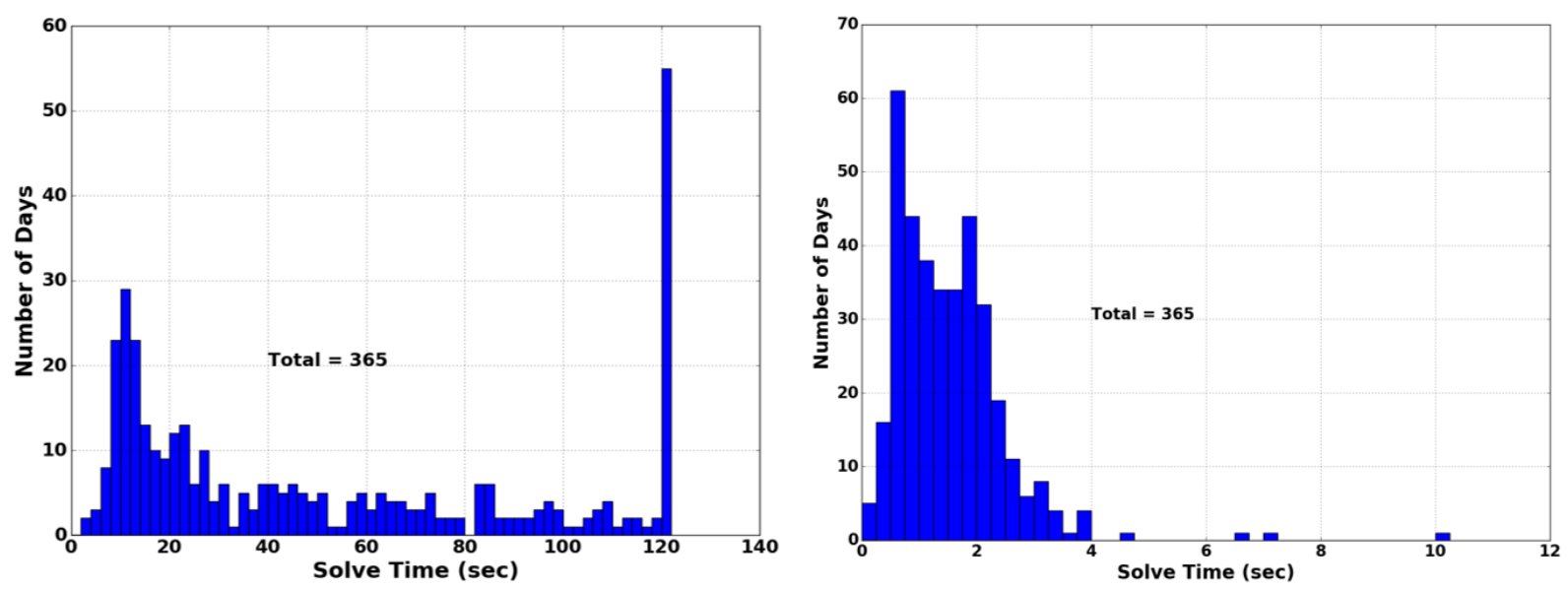

Figure 29. The distribution of dispatch problem solve times for a year's worth of time periods for the original (left) and Version A (right) formulations

\subsubsection{Hybrid system design optimization}

Shorter dispatch solve times allow us to focus on hybrid system design optimization. The evaluation of a particular hybrid design while also accounting for the corresponding dispatch strategy is computationally expensive, e.g., 10 - 15 minutes per design, and the underlying optimization model is impossible to express explicitly. Therefore, we optimize the design of CSP-PV hybrid system using a derivative-free black-box optimization approach.

Black-box optimization involves only a query from the solver related to the objective function value given a set of variable values, rather than, for example, possessing the ability to obtain gradient information or knowledge of the analytic form of the model. Black-box optimization solvers make a trade-off between exploitation and exploration. Exploitation consists of probing a small region to find a better solution than the solver currently has, and is referred to as local search. Exploration consists of probing a larger space to find a new region with solutions better than the incumbent, and is referred to as global search. Exploration can result in convergence to the globally optimal solution, but is computationally expensive due to the number of function evaluations required to explore the entire feasible region. To effect these implicit solves, we use ORBIT (Optimization by Radial Basis function Interpolation in Trust-regions) (Wild, Regis, and Shoemaker), which has been shown to converge to a local minimum with a small number of function evaluations. To overcome the challenges associated with local search methods, we sample the design space to provide a "good" initial solution to the solver. We use a Latin hypercube with a maximin secondary criterion. However, alternate sampling techniques exist, and future work will test solution sensitivity to the sampling technique.

Sampling provides an initial solution as a means to explore the design space before preforming a local search, but can be computationally expensive. On the other hand, it can be done in parallel and, in our case, requires a SAM simulation to uniquely call a dispatch optimization model instance and wait for the corresponding solution. To this end, we have altered SAM to allow the user to provide a unique identification string that corresponds to a temporary dispatch optimization model file. SAM and dispatch optimization use the unique identification string to communicate during the annual model run without interference from other, parallel runs. After a run is complete, a cleaning procedure deletes all the uniquely identified files. Although its efficiency can be improved, this approach has proven effective for our testing, and required the least development time and fewest changes to SAM. To optimize hybrid system design, a modified PPA metric is used as the objective function, shown in Equation (3.52). 


$$
\min \operatorname{PPA}(\mathcal{S})+\alpha \mid \max \text { flux }-\max \text { obs. } \operatorname{flux}(\mathcal{D}) \mid
$$

where PPA is the power purchase agreement which is a function of the annual run $\mathcal{S}, \alpha$ is a weighting factor to scale the flux difference to PPA, max flux is the maximum allowable receiver flux specified by user, and max obs. flux is the maximum observed flux on the receiver during a given run, which is a function of CSP design. The second term penalizes maximum flux deviations from allowable flux, which ensures that the CSP solar field and receiver are not under- or over-utilized.

\subsubsection{Hybrid system case study}

The hybrid system of interest is grid-limited and requires the dispatch of individual sub-systems (i.e., power cycle, photovoltaics, and battery) to ensure that the power limit of $310 \mathrm{MW}_{\mathrm{e}}$ is not exceeded. The CSP sub-system consists of two twin molten salt power tower CSP plants, each with a power cycle capable of $163 \mathrm{MW}_{\mathrm{e}}$ output with 12 hours, or $4716 \mathrm{MWh}_{\mathrm{t}}$, of thermal storage and a receiver capable of $565 \mathrm{MW}_{\mathrm{t}}$ production. The $\mathrm{PV}$ sub-system consists of a single-axis tracking PV field capable of $325 \mathrm{MW}_{\mathrm{dc}}$ production with a maximum inverter power output of 270 $\mathrm{MW}_{\mathrm{ac}}$. The battery sub-system has a capacity of $150 \mathrm{MWh}_{\mathrm{e}}$ electrical energy with a maximum discharge rate of $1 \mathrm{C}$ (i.e., $150 \mathrm{MW}_{\mathrm{e}}$ ). Table 26 summarizes design parameters and defines operational limits. Given solar resource and receiver-rated thermal power, SolarPILOT (Wagner and Wendelin) generates the CSP heliostat field layout for the two locations.

\subsection{Full-Factorial Experiment}

We characterize the performance of the dispatch model $(\mathcal{M})$ for a fixed hybrid system design and operating costs using a full-factorial experiment. The design of experiments consists of two factors, each with two levels. The two factors chosen for this experiment are location of the CSP-PV hybrid plant and the electricity market in which the plant is being dispatched.

\section{Plant Location}

The location of the hybrid plant is important with respect to its performance and profitability. Geographic location determines the solar resource availability for electricity generation. For our case study, we choose northern Chile (N. Chile) and southern Nevada (S. Nevada) as the two levels for the location factor, the former location owing to its solar resource and our industry partners' interest in the development of hybrid plants within this region and the latter region owing to its high solar resource and the availability of sub-hourly (i.e., one-minute time scale) solar data (Andreas and Stoffel).

Figure 3.30(a) compares the direct normal irradiance for N. Chile and S. Nevada using a sorted histogram. The yearly totals for the three solar irradiance resources are given in Table 27. N. Chile has a higher solar resource than S. Nevada because the area has low atmospheric attenuation. S. Nevada has higher annual diffuse irradiance because there are more days with cloud cover than N. Chile. CSP systems can only use direct normal irradiance, while PV systems can generate electricity from both direct normal and diffuse horizontal irradiance.

\section{Electricity Markets}

The electricity market in which the plant bids into affects the dispatch strategy due to the time-of-use price of electricity. For our factorial experiment, we choose the N. Chile spot market (NC spot) and the 2016 Pacific Gas \& Electric (PG\&E) full capacity deliverability market as the two levels for the market factor, the former owing to our industry partner's interest, and the latter owing to its simple design and the possibility that a plant in southern Nevada may be designed to dispatch against such a market.

Figure 3.30(b) compares the two markets using a sorted histogram of normalized electricity prices. Both markets follow a similar price trend. However, the PG\&E market has more high-price and more highly priced hours relative to those in the $\mathrm{NC}$ spot market. 


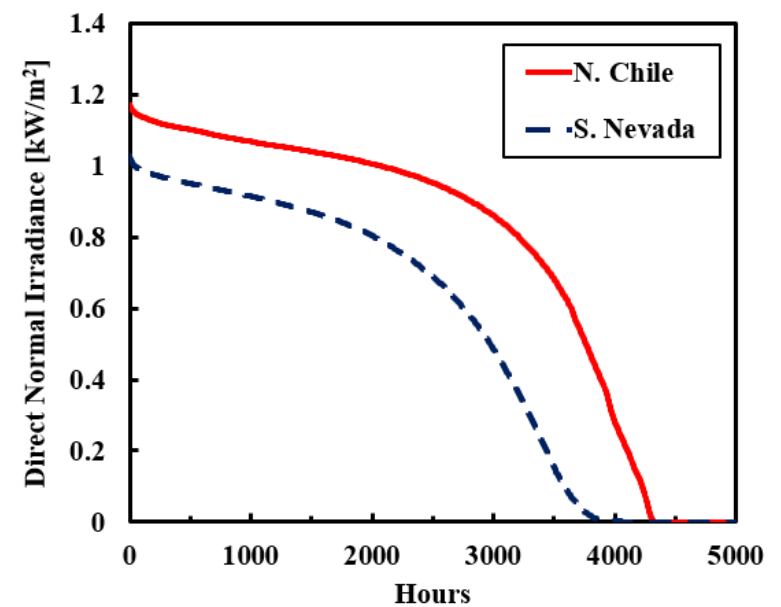

(a) Sorted histogram of the direct normal irradiance for N. Chile and S. Nevada

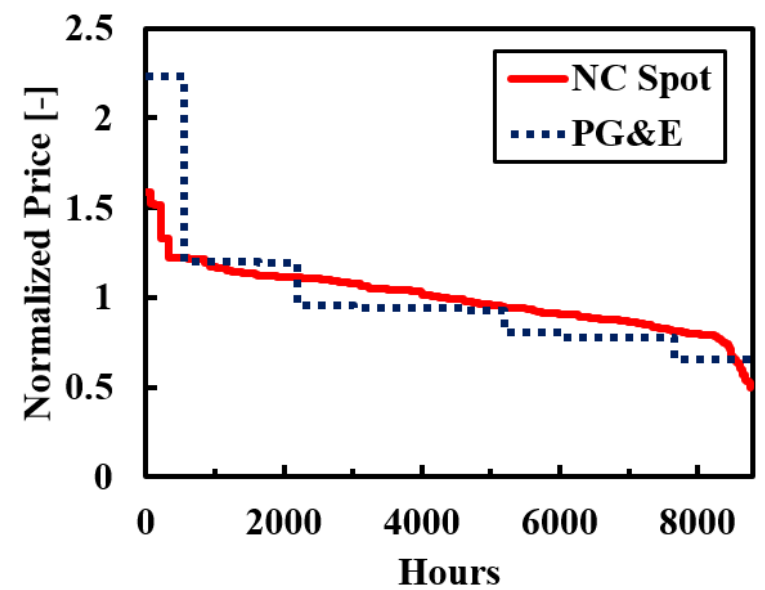

(b) Sorted histogram of the normalized prices for N. Chile spot (NC spot) and Pacific Gas \& Electric (PG\&E) markets.

Figure 30. Sort histograms to compare direct normal irradiance and market structures of the full-factorial experiment

Figure 31 depicts the time-of-use variation of the NC spot and PG\&E markets. The NC spot market exhibits monthly and week-versus-weekend variation in the electricity price, shown in Figure 3.31(a). Generally, high-price time periods occur at the end of the solar day during the winter and spring, i.e., July through December. During the fall (April through June), the NC spot market possesses relatively flat prices- either slightly above or below the annual average. The PG\&E market has only seasonal variation, with the high-price time periods occurring between the hours of 4 and 9 p.m. The highest values occur during the months of July, August, and September.

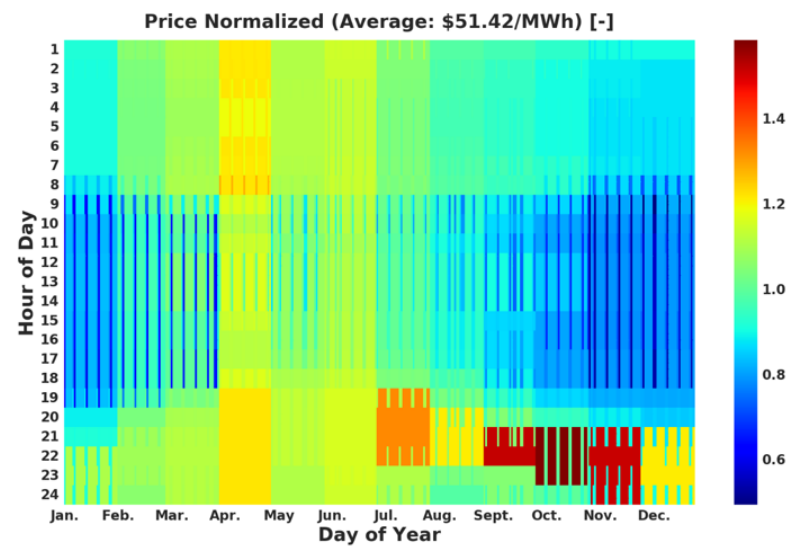

(a) NC spot market normalized to an average electricity price of $\$ 51.42 / \mathrm{MWh}$

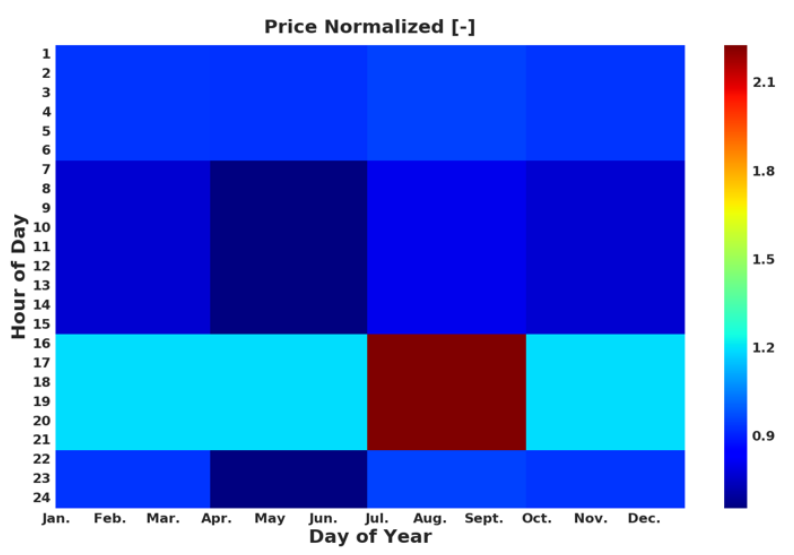

(b) PG\&E market normalized by the power purchase agreement.

Figure 31. Time-of-use variation of electricity prices for the Northern Chile spot (NC spot) and Pacific Gas \& Electric (PG\&E) market.

Due to the seasonal variation in both markets and the difference in seasons at the same point in time in the northern and southern hemispheres, we shift the one of the markets by six months when evaluating the location-market combinations of $\mathrm{N}$. Chile in a PG\&E market and S. Nevada in a NC spot market. This seasonal shift was done by 
appending the first 181 days (i.e., first six months) of the year to the end of the last 184 days (i.e., last six months) of the year. This method does not preserve the monthly transitions that were present in the original price structure. However, it does allow for comparison between the original markets because this shift does not change the number of time periods that exhibit a particular price.

\subsection{Solve Times and Solution Quality}

Each location-market combination in the full-factorial experiment requires the solution of 365 instances of the dispatch problem to produce an annual schedule. We limit each instance of the dispatch problem to 8 threads on the computing system, solving either to an optimality gap of $1 \times 10^{-3}$ or to a specified time limit, whichever occurs first. Applying the described solution techniques to $(\mathcal{M})$ yields a solution time for the year-long solves of about 34 minutes, an 86 to $93 \%$ improvement compared to the original formulation solution time without considering the three methods we mention above.

\subsection{Annual Plant Performance}
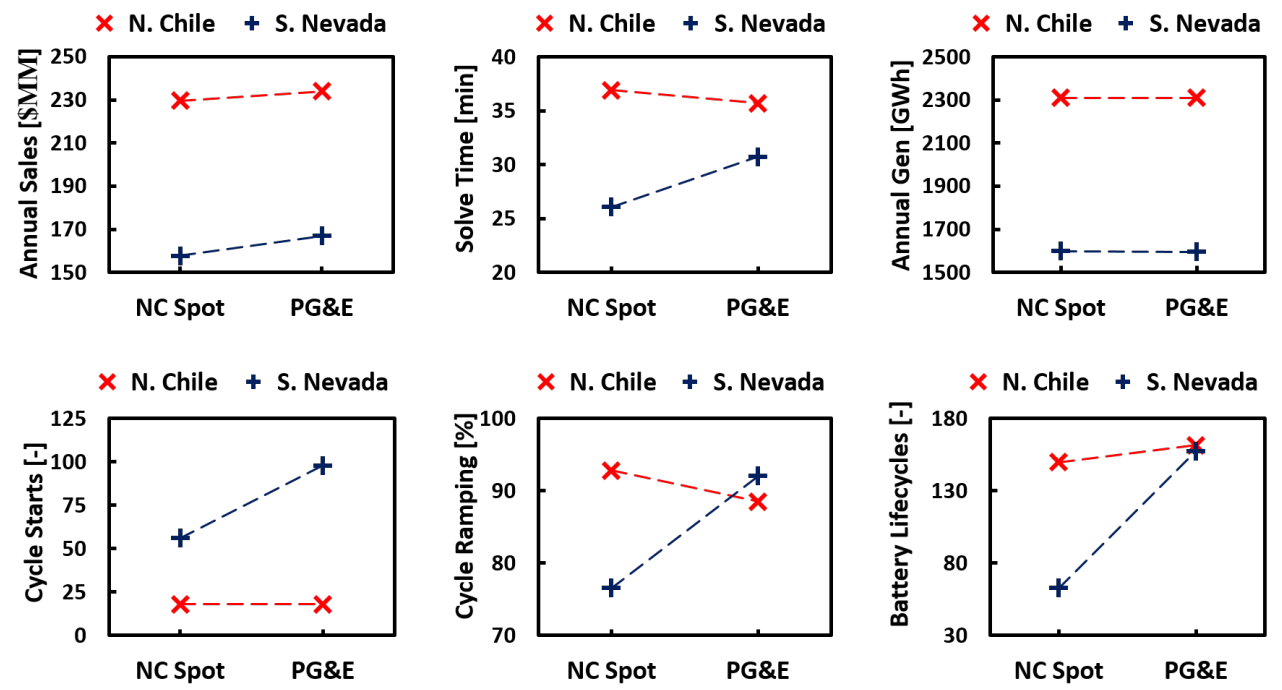

Figure 32. Annual solution attributes for the full-factorial experiment; annual sales, solve time, annual generation, cycle starts, cycle ramping, and battery lifecycles.

An annual schedule of the CSP-PV hybrid plant allows us to understand the performance of a particular system design in a given location and under certain market conditions. Figure 32 plots six annual dispatch schedule responses for the full-factorial experiment.

We calculate annual sales as the sum over all time periods within a year of the product of the energy generation of the hybrid system, the price multiplier, and an assumed PPA price of $\$ 0.1 / \mathrm{kWh}$. This response plot shows that the PG\&E market results in greater sales than the NC spot market owing to the higher price multiplier during highprice time intervals in the PG\&E market relative to those for the NC spot market. However, the PG\&E market has a greater effect on this plant design in S. Nevada than in N. Chile, owing to the lower solar resource in S. Nevada and optimization having a greater effect by dispatching generation during high-revenue time intervals.

Figure 32 depicts annual solution time and generation responses; the former show that N. Chile annual dispatch strategies require more computational time, consistent with the results reported above. Annual generation shows that there is little-to-no interaction between generation and the market factor because the price at which electricity is sold should not affect how much electricity the plant can generate throughout the year. However, the annual generation 
plot shows the impact of the two locations. Due to the greater solar resource in N. Chile, this plant design produces about $700 \mathrm{GWh}$ more electricity than the same plant in S. Nevada.

The responses cycle starts and cycle ramping demonstrate how the power cycles would be operated. Cycle ramping is the daily average percentage of the rated cycle power the cycle ramps up and down, e.g., 100\% represents the power cycle ramping from zero to rated power every day. The hybrid plant power cycle in $\mathrm{N}$. Chile would only need to start up about 20 times in a given year. However, the same plant in S. Nevada would need to start the power cycle 50 to 100 times annually, depending on the market, which could cause more wear and tear on the cycle, resulting in an increase in maintenance costs. Interestingly, the PG\&E market requires more ramping than the NC spot market for the S. Nevada location, but less for the N. Chile location.

The last response plotted is battery lifecycles, important to track because they help determine if the battery must be replaced during the 25- to 30-year life of the these projects, and calculated based on the current throughput from the battery, $i_{t}^{-}$and $i_{t}^{+}$, using the following equation:

$$
b^{C}=\sum_{t=1}^{\mathcal{T}} \frac{\Delta\left(i_{t}^{-}+i_{t}^{+}\right)}{C^{B}}
$$

where $C^{B}$ is the capacity of the battery. Of the four cases, the S. Nevada with in the NC spot market possesses the lowest number of battery lifecycles owing to the relatively flat price structure in the NC spot market and less abundant solar resource in S. Nevada. The battery is used in N. Chile to capture excess solar energy that would otherwise be curtailed. However, the hybrid system could be redesigned to include little-to-no solar curtailment.

\subsection{Software development}

The goal of this subtask is to develop a software interface that allows users to access the optimization, design, and operations algorithms that have been produced in this project, and to develop improvements in the implementation that reduce the computation time required to obtain solutions for users. The user interface (UI) must satisfy a comprehensive list of requirements, including:

- Provide access to model functionality

- Provide data importing and exporting tools

- Allow flexibility in the sequence of modeling steps to accommodate different development needs

- Be able to store projects and load them for later use

- Be readily accessible for users with an engineering or operations background

- Provide data visualization tools

- Run on different operating systems

- Document functionality

- Be able to interact with external engineering models

Several different UI approaches are possible, and common ones include a web interface, standalone desktop application, script-based interface, command line interface, application programming interface, or a spreadsheet interface. Each option offers certain advantages and limitations, and the selection of the most appropriate interface for this project must weigh these aspects, as summarized in Table 28.

After weighing the choices, we selected the script UI as the best option. This approach offers the wide flexibility that is needed for our application, while remaining accessible to most users and providing some guidance in terms of workflow. The script UI is implemented as a standalone application that can be installed on a local machine and not require a reliable internet connection, which may be challenging in remote or lesser-developed locations. 
The working name for the software is Design, Analysis, and Operations Toolkit, or "DAO-Tk." The acronym echoes the Chinese word Dao (or Tao), which has one of several interpretations meaning "a holistic approach," roughly describing the primary goal of this project with respect to CSP modeling.

The application is developed in C++ using the wxWidgets interface toolkit. The scripting language is NREL's $L K$, which has been developed specifically for simulation-based software and is used in SAM, SDKTool, SolTrace, and SolarPILOT ${ }^{\mathrm{TM}}$. LK offers many built-in functions for processing files and executing common mathematical functions, and it provides flexible data types such as tables and arrays that expedite script development. The LK language is syntactically similar to $\mathrm{C}++$, but Python-like data structures and built-in functions improve usability. 
Table 10. Mean field reflectivity (Refl.), mean number of mirror replacements (NR), and point and interval estimates for the mean relative loss reduction (LR) as a function of replacement and washing policies

$\begin{array}{lccccc}\text { Replacement/Wash Policy } & \text { Crews } & \text { Refl. } & \text { NR } & \text { Mean LR } & \text { 95\% CI LR } \\ \text { None/Uniform } & 3 & 0.9127 & 0 & \text { NA } & \text { NA } \\ \text { None/Uniform } & 5 & 0.928 & 0 & 0.1750 & {[0.1729,0.1771]} \\ \text { Uniform/Uniform } & 5 & 0.9473 & 11,116 & 0.2534 & {[0.2441,0.2627]} \\ \text { Individual/Uniform } & 5 & 0.9477 & 11,677 & 0.2507 & {[0.2475,0.2539]} \\ \text { Individual/Individual } & 5 & 0.9478 & 11,678 & 0.2521 & {[0.2489,0.2553]}\end{array}$

Table 11. Definitions for the power cycle failure formulation.

\begin{tabular}{|c|c|c|}
\hline \multicolumn{3}{|l|}{ Set } \\
\hline $\mathcal{T}$ & & set of all time steps in the time horizon \\
\hline \multicolumn{3}{|c|}{ Parameters } \\
\hline$F_{t}^{c a p}$ & & relative capacity at time $t$ \\
\hline$F_{t}^{e f f}$ & & relative efficiency at time $t$ \\
\hline$\eta$ & & denotes efficiency parameter \\
\hline$Q^{u}$ & $\mathrm{~kW}_{t}$ & cycle thermal power capacity \\
\hline \multicolumn{3}{|l|}{ Variables } \\
\hline$x_{t}$ & $\mathrm{~kW}_{t}$ & cycle thermal power consumption at time $t$ \\
\hline$\dot{w}_{t}$ & $\mathrm{~kW}_{e}$ & electrical power generation at time $t$ \\
\hline$y_{t}$ & & 1 if cycle is generating electric power at time $t 0$ otherwise \\
\hline \multicolumn{3}{|l|}{ Function } \\
\hline$f\left(x_{t}, y_{t}, \eta\right)$ & $\mathrm{kW}_{e}$ & maximum power generation function \\
\hline
\end{tabular}

Parameters
$x_{t}^{\text {fixed }}:$ previously determined thermal consumption at time $t \quad\left[\mathrm{~kW}_{\text {thermal }}\right]$
failure_period : first period with a failure or maintenance event

Table 12. Factors and levels of first experiment.

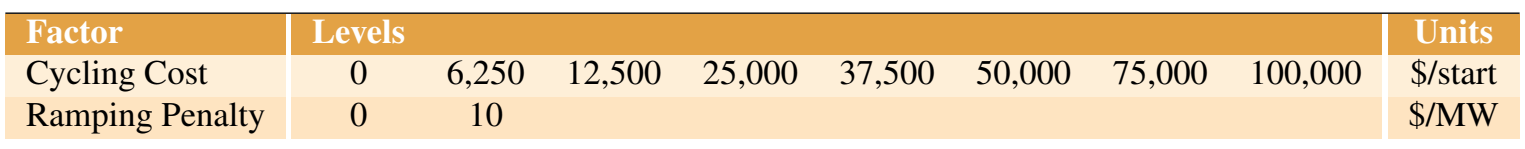


Table 14. Variables used in (R).

\begin{tabular}{|c|c|c|}
\hline $\begin{array}{l}\text { Symbol } \\
\text { Contin }\end{array}$ & $\begin{array}{l}\text { Units } \\
\text { us }\end{array}$ & Description \\
\hline$x_{t}$ & $\mathrm{~kW}_{t}$ & Cycle thermal power consumption at $t$ \\
\hline$\dot{w}_{t}$ & $\mathrm{~kW}_{e}$ & Electrical power generation at $t$ \\
\hline$\dot{w}_{t}^{\delta}$ & $\mathrm{kW}_{e}$ & Positive change in electricity production at $t$ \\
\hline$x_{t}^{r}$ & $\mathrm{~kW}_{t}$ & Thermal power delivered by the receiver at $t$ \\
\hline$x_{t}^{r s u}$ & $\mathrm{~kW}_{t}$ & Receiver start-up power consumption at $t$ \\
\hline$u_{t}^{r s u}$ & $\mathrm{~kW}_{t}-\mathrm{hr}$ & Receiver start-up energy inventory at $t$ \\
\hline$u_{t}^{c s u}$ & $\mathrm{~kW}_{t}-\mathrm{hr}$ & Cycle start-up energy inventory at $t$ \\
\hline$s_{t}$ & $\mathrm{~kW}_{t}-\mathrm{hr}$ & TES reserve quantity at $t$ (auxiliary variable) \\
\hline \multicolumn{3}{|l|}{ Binary } \\
\hline$y_{t}^{r}$ & & 1 if receiver is generating "usable" thermal power at time $t ; 0$ otherwise \\
\hline$y_{t}^{r s u}$ & & 1 if receiver is starting up at time $t ; 0$ otherwise \\
\hline$y_{t}^{r s b}$ & & 1 if receiver is in standby mode at time $t ; 0$ otherwise \\
\hline$y_{t}^{r s d}$ & & 1 if receiver shut down at time $t ; 0$ otherwise \\
\hline$y_{t}^{c S u}$ & & 1 if cycle is starting up at time $t ; 0$ otherwise \\
\hline$y_{t}^{c s b}$ & & 1 if cycle is in standby mode at time $t ; 0$ otherwise \\
\hline$y_{t}^{c s d}$ & & 1 if cycle is shutting down at time $t ; 0$ otherwise \\
\hline$y_{t}$ & & 1 if cycle is generating electric power at time $t ; 0$ otherwise \\
\hline$y_{t}^{r s u p}$ & & $\begin{array}{l}1 \text { if receiver is starting up at time } t \text { and was not in standby mode at time } \\
t-1 ; 0 \text { otherwise }\end{array}$ \\
\hline$y_{t}^{r h s p}$ & & $\begin{array}{l}1 \text { if receiver is starting up at time } t \text { and was in standby mode at time } t-1 ; 0 \\
\text { otherwise }\end{array}$ \\
\hline$y_{t}^{\text {csup }}$ & & $\begin{array}{l}1 \text { if cycle is starting up at time } t \text { and was not in standby mode at time } t-1 \text {; } \\
0 \text { otherwise }\end{array}$ \\
\hline$y_{t}^{c h s p}$ & & $\begin{array}{l}1 \text { if cycle is starting up at time } t \text { and was in standby mode at time } t-1 ; 0 \\
\text { otherwise }\end{array}$ \\
\hline
\end{tabular}




\section{Table 15. Case study plant design and control parameters}

$\begin{array}{lcc}\text { Parameter } & \text { Units } & \text { Value } \\ \text { Gross electrical output } & \text { MWe } & 115 \\ \text { Cycle design efficiency } & \% & 41.2 \\ \text { Cycle design thermal input } & \mathrm{MWt} & 278.1 \\ \text { Cycle maximum output } & \mathrm{MWe} & 120.75 \\ \text { Cycle minimum output } & \mathrm{MWe} & 28.75 \\ \text { Cycle start-up energy } & \mathrm{MWt}-\mathrm{hr} & 57.5 \\ \text { Cycle start-up time } & \mathrm{hr} & 0.5 \\ \text { Cycle standby consumption } & \mathrm{MWt} & 23 \\ \text { Receiver max. output (relative*) } & - & 1.2 \\ \text { Receiver min. output (relative) } & - & 0.25 \\ \text { Receiver start-up energy (relative) } & - & 0.25 \\ \text { Receiver start-up time (relative) } & - & 0.2 \\ \text { Receiver HTF temperature } & { }^{\circ} \mathrm{C} & 574 \\ \text { Heat rejection technology } & - & \text { Air cooled } \\ \text { Heliostat size } & \mathrm{m}^{2} & 144.4 \\ \text { Maximum receiver flux } & \mathrm{kW} / \mathrm{m}^{2} & 1,000 \\ \text { Hours of TES } & \mathrm{hr} & 1, \ldots, 18 \\ \text { Solar multiple } & - & 0.8, \ldots, 3\end{array}$

*Relative to receiver thermal input design point.

Table 16. Characteristics for each market scenario in which PPA price is at a minimum value, both for heuristic $(\mathrm{H})$ and optimized $(\mathrm{O})$ dispatch.

\begin{tabular}{|l|cc|cc|cc} 
Market & \multicolumn{2}{|c|}{ Solar mult. } & \multicolumn{2}{c|}{ Hours TES } & \multicolumn{2}{c|}{ PPA price } \\
scenario & H & O & H & O & H & O \\
\hline Two-tier & 2.2 & 2.0 & 5 & 7 & 8.97 & 7.71 \\
Pool price & 2.2 & 2.2 & 8 & 9 & 9.66 & 8.75 \\
Fixed daytime & 1.8 & 2.0 & 4 & 6 & 13.30 & 12.75 \\
SAM default & 2.6 & 2.6 & 12 & 14 & 10.41 & 10.09
\end{tabular}


Table 18. Decision variables

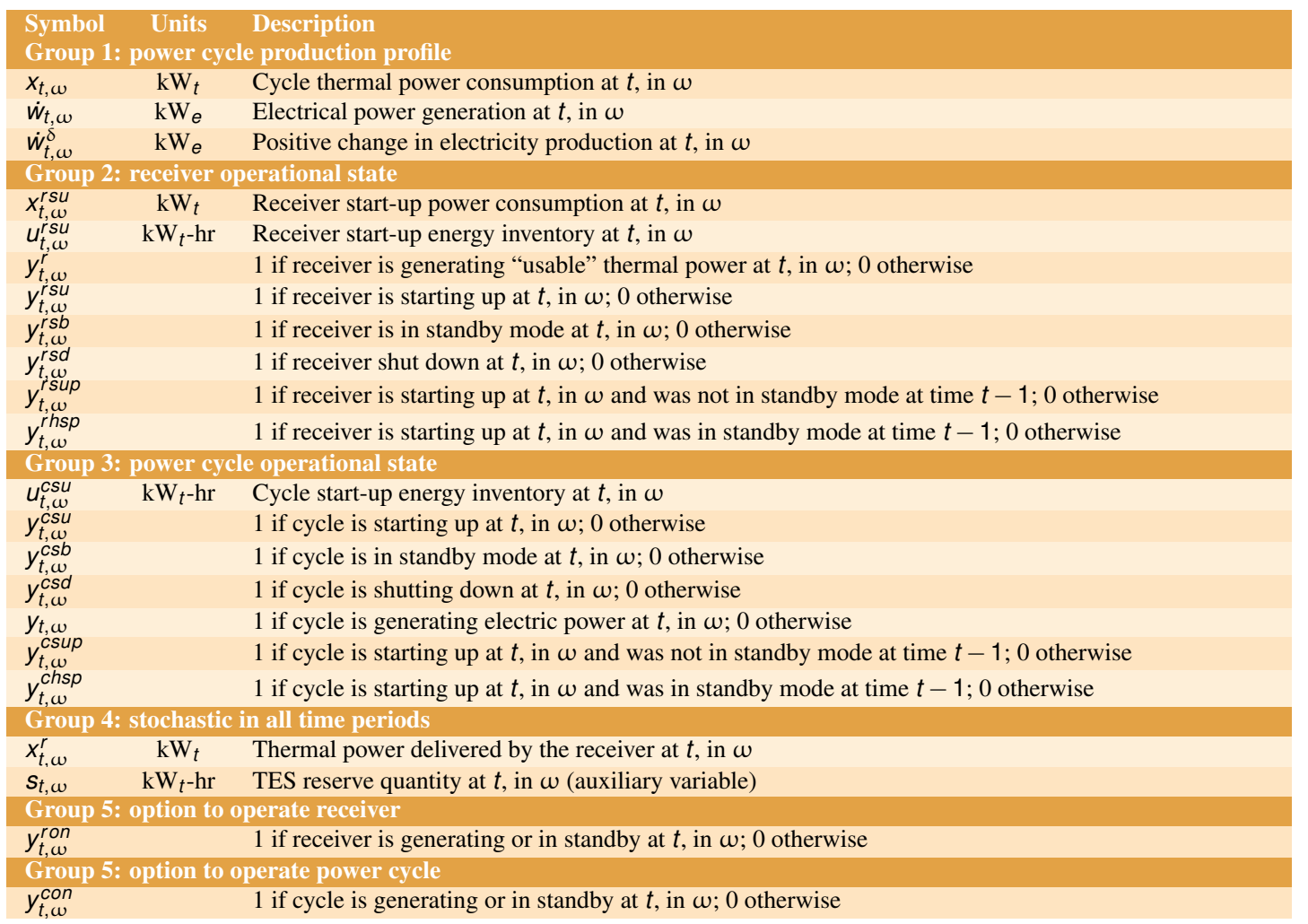

$\begin{array}{lll} & \text { Revenue }(\$) & \text { Revenue }(\%) \\ \text { EV } & 22,589,292 & - \\ \text { SP } & 25,922,107 & 14.8 \\ \text { Policy } & 29,349,125 & 29.9\end{array}$

Table 19. Revenue for one year derived from the expected value problem (EV), the stochastic program (SP), and the policy approach using synthetic data. The percentage values are the increase over EV.

\begin{tabular}{|c|c|c|c|c|c|c|}
\hline \multicolumn{7}{|c|}{ Revenue } \\
\hline & \multicolumn{2}{|l|}{2010} & \multicolumn{2}{|l|}{2011} & \multicolumn{2}{|l|}{2012} \\
\hline & $\$$ & $\%$ & $\$$ & $\%$ & $\$$ & $\%$ \\
\hline $\mathrm{EV}$ & $24,789,873$ & - & $28,963,500$ & - & $29,978,444$ & - \\
\hline SP & $26,158,645$ & 5.6 & $30,350,246$ & 4.8 & $31,269,338$ & 4.3 \\
\hline Policy & $27,064,016$ & 9.2 & $30,696,800$ & 6.0 & $31,295,170$ & 4.4 \\
\hline
\end{tabular}

Table 20. Revenue derived from the expected value problem (EV), the stochastic program (SP), and the policy approach using historical data from Hanford, California. The percentage values are the increase over EV. 
(a) Relative improvements (\%) in PPA prices over historical average DNI for SOLRAD data for Hanford CA. Here, we use the nominal dispatch model, calculate the PPA price in SAM using 24-hour time increments, and energy prices according to default SAM multipliers.

\begin{tabular}{|l|lll|}
\hline & \multicolumn{3}{|c|}{ Test Years } \\
& 2010 & 2011 & 2012 \\
\hline Observed DNI & 21.09 & 18.16 & 17.80 \\
Point forecast & 16.52 & 13.58 & 14.29
\end{tabular}

(b) Relative gap (\%) in PPA prices over perfect information on DNI for SOLRAD data for Hanford, CA. Both the policy approach and the nominal approach use model (3.49) with $\lambda>0$ and $\lambda=0$, respectively, and both approaches benefit from the conditional DNI point forecast. The final column's values for 2012 mean that a perfect information PPA value grows by $5.30 \%$ under the policy approach and grows by $11.18 \%$ under the nominal dispatch model.

\begin{tabular}{|l|lll|} 
& \multicolumn{3}{|c}{ Test Years } \\
& 2010 & 2011 & 2012 \\
\hline Policy & 9.87 & 8.99 & 5.30 \\
Nominal & 14.71 & 15.22 & 11.18 \\
\hline
\end{tabular}

Table 22. Relative gap (\%) in PPA prices over perfect information on RTM15 price forecasts for SOLRAD data for Hanford, California. Both the price point forecast and the historical mean use model (3.49) with $\lambda=0$, and both approaches benefit from the conditional DNI point forecast. The values mean that relative to a perfect information PPA price, the price grows by $9.60 \%$ under the conditional point forecast and grows by about $31 \%$ under the historical mean.

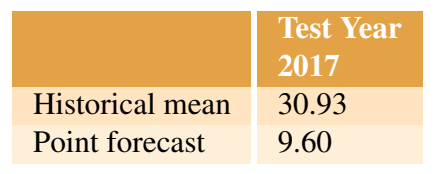

Table 23

(a) Relative gap (\%) in PPA prices over perfect information on DNI for 2017 SOLRAD data for Hanford, California.

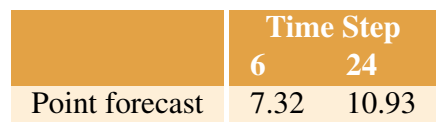

(b) Relative gap (\%) in PPA prices over perfect information on RTM15 for 2017 SOLRAD data for Hanford, California.

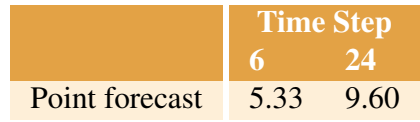

Table 24. Comparison of annual hybrid simulation metrics between the dispatch model with objective function (3.50) and (3.51). ${ }^{\dagger}$ Solve times are based on Quad-Quad core CPUs at $2.93 \mathrm{GHz}$ 32GB RAM 1TB HDD hardware.

$\begin{array}{lcc} & \text { Objective }(\mathbf{3 . 5 0}) & \text { Objective (3.51) } \\ \text { Generation [GWhe] } & 632.9 & 638.6 \\ \text { PPA [\$/kWh] } & 11.66 & 11.54 \\ \text { PV Curtailment [\%] } & 24.9 & 0.3 \\ \text { Cycle Starts [-] } & 86 & 37 \\ \text { Battery Life Cycles [-] }^{\text {Average Day Solve Time [sec] }}{ }^{\dagger} & 259.6 & 156.7 \\ & 2.04 & 1.01\end{array}$

Table 25. CSP-PV hybrid sub-system operating cost ranges based on literature (references provided in brackets) or engineering approximations and the current value of each cost, given in units of $\$ / M W h$.

$\begin{array}{lccc}\text { Sub-system } & \text { Lower } & \text { Upper } & \text { Setting } \\ \text { Receiver/CSP field (thermal) } & 3.25 & 9.33 & 5.00 \\ \text { Photovoltaic (Whaley) } & 1.00 & 8.00 & 1.00 \\ \text { Power Cycle (Kumar et al.) } & 0.85 & 3.24 & 2.00 \\ \text { Battery (Akhil et al.) } & 1.10 & 5.50 & 3.00\end{array}$


Table 26. Case study plant design

$\begin{array}{lcc}\text { Parameter } & \text { Units } & \text { Value } \\ \text { CSP system: } & \mathrm{MW}_{\mathrm{t}} & 393 \\ \text { Cycle design thermal input } & \mathrm{MW}_{\mathrm{e}} & 163 \\ \text { Cycle maximum gross output } & \mathrm{MW}_{\mathrm{e}} & 37.5 \\ \text { Cycle minimum gross output } & \mathrm{MWh}_{\mathrm{t}} & 197 \\ \text { Cycle start-up energy consumption } & \mathrm{hr} & 0.5 \\ \text { Cycle minimum start-up time } & \mathrm{MW}_{\mathrm{t}} & 78.6 \\ \text { Cycle standby consumption } & \mathrm{MW}_{\mathrm{t}} & 565 \\ \text { Receiver maximum/design thermal output } & \mathrm{MW}_{\mathrm{t}} & 141 \\ \text { Receiver minimum output } & \mathrm{MWh}_{\mathrm{t}} & 141 \\ \text { Receiver start-up energy } & \mathrm{hr} & 0.2 \\ \text { Receiver minimum star-up time } & \mathrm{MWh}_{\mathrm{t}} & 4716 \\ \text { Thermal storage maximum charge } & & \\ \text { PV and battery system: } & \mathrm{MW}_{\mathrm{dc}} & 325 \\ \text { PV field capacity } & \mathrm{MW}_{\mathrm{ac}} & 270 \\ \text { PV inverter maximum output } & \mathrm{MWh}_{\mathrm{e}} & 150 \\ \text { Battery capacity } & \mathrm{MW}_{\mathrm{e}} & 150 \\ \text { Battery maximum power output } & & \\ \text { Whole system: } & \mathrm{MW}_{\mathrm{e}} & 310 \\ \text { Grid maximum power limit } & & \end{array}$

Table 27. Solar global horizontal, direct normal, and diffuse horizontal irradiance year totals for N. Chile and S. Nevada.

\begin{tabular}{l|ccc} 
& $\begin{array}{c}\text { Global Horizontal } \\
{\left[\mathrm{kWh} / \mathrm{m}^{2} / \text { year] }\right.}\end{array}$ & $\begin{array}{c}\text { Direct Normal } \\
{\left[\mathrm{kWh} / \mathrm{m}^{2} / \mathrm{year}\right]}\end{array}$ & $\begin{array}{c}\text { Diffuse Horizontal } \\
{\left[\mathrm{kWh} / \mathrm{m}^{2} / \text { year] }\right.}\end{array}$ \\
\hline N. Chile & 3782.1 & 2730.4 & 353.4 \\
S. Nevada & 2682.9 & 2083.2 & 490.4
\end{tabular}


Table 28. Comparison of advantages and disadvantages for different user interface options

\begin{tabular}{|c|c|c|}
\hline Interface & Advantages & Disadvantages \\
\hline Web & $\begin{array}{l}\text { - Easily updated } \\
\text { - Familiar to most users } \\
\text { - Can be readily customized } \\
\text { - Many development tools available }\end{array}$ & $\begin{array}{l}\text { - Requires reliable connection to server } \\
\text {-Data download and upload required } \\
\text { - Connections prone to security risk } \\
\text { - Requires hosting service }\end{array}$ \\
\hline Desktop app & $\begin{array}{l}\text {-Easily controlled workflow } \\
\text { - Clear options for input values } \\
\text { - Self-contained distribution }\end{array}$ & $\begin{array}{l}\text {-Lacks flexibility for use in } \\
\text { different projects } \\
\text {-Large development effort } \\
\text { - Not easily automated }\end{array}$ \\
\hline Script & $\begin{array}{l}\text { - Highly flexible } \\
\text {-Enabled complete automation } \\
\text { of workflow } \\
\text { - Takes advantage of existing } \\
\text { LK language }\end{array}$ & $\begin{array}{l}\text { - Helpful to have programming knowledge } \\
\text {-Does not provide guided workflow } \\
\text { - May not interface seamlessly with } \\
\text { standard programming languages }\end{array}$ \\
\hline Command line & $\begin{array}{l}\text { - Allows interactive use } \\
\text { - Highly flexible } \\
\text {-Can be automated via shell scripts }\end{array}$ & $\begin{array}{l}\text {-Least guidance offered to user } \\
\text { - Requires programming knowledge } \\
\text {-Limited options for in-program } \\
\text { data visualization }\end{array}$ \\
\hline Language API & $\begin{array}{l}\text {-Highly flexible } \\
\text { - Facilitates use of full features of } \\
\text { calling language } \\
\text { - Low development effort }\end{array}$ & $\begin{array}{l}\text {-Many opportunities for errors in } \\
\text { making data connections } \\
\text { - Requires full programming knowledge } \\
\text { - No software-provided visualization }\end{array}$ \\
\hline Spreadsheet & $\begin{array}{l}\text {-Can readily implement } \\
\text { formulas for inputs } \\
\text { •Exposes all data } \\
\bullet \text { Most broadly usable }\end{array}$ & $\begin{array}{l}\text { - Notoriously confusing } \\
\text { workflow management } \\
\text { •Exposes all data } \\
\text { - Manually intensive usage }\end{array}$ \\
\hline
\end{tabular}




\subsubsection{Computational efficiency}

Here, we detail a collection of four methods that we implement to improve the time required to obtain model outputs for a given design. In what follows, we describe these methods and, if needed, the names of the variables associated with activating these improvements when evaluating the objective value of a design.

\subsubsection{Data clustering}

Net electricity generation $\left(W^{\text {net }}\right)$ and revenue $(R)$ from a full annual hourly time series simulation in SAM can be represented by equation 3.53 , in which the functional dependence of the hourly net electricity generation $\left(w_{t}^{\text {net }}\right)$ on the hourly electricity price $\left(P_{t}\right)$ arises as a result of the dispatch optimization algorithm, and the hourly solar field availability factor $\left(s_{t}^{\text {avail }}\right)$ combines information from the heliostat failure, optical degradation, and soiling models.

$$
W^{\text {net }}=\sum_{t=1}^{8760} w_{t}^{\text {net }}\left(\text { weather }_{t}, P_{t}, s_{t}^{\text {avail }}\right) \quad, \quad R=\sum_{t=1}^{8760} P_{t} w_{t}^{\text {net }}\left(\text { weather }_{t}, P_{t}, s_{t}^{\text {avail }}\right)
$$

We seek to reduce the computational expense of the full annual performance simulation in SAM by grouping daily weather and pricing profiles into $N_{c}$ clusters, each containing samples of $N_{d}$ consecutive days which share similar characteristics such that simulation of hourly electricity generation from one sample per cluster (the exemplar) can effectively represent all samples within the cluster. Annual electricity generation and revenue can then be approximated from the clustered simulations via equations 3.54 and 3.55, in which $W_{j}^{\text {net }}$ is the total generation over the $N_{d}$-day period serving as the exemplar for cluster $j, t_{1, j}$ is the first time point in this period, and $f_{j}$ is the weighting factor for cluster $j\left(0<f_{j}<1\right)$ defined by equation 3.56. Weighting factors are calculated from elements of the partition matrix $\left(\omega_{i, j}\right)$, which describes the fraction of data point $i$, which, in turn, is allocated to cluster $j$. In this context, a "data point" is defined as a single group of $N_{d}$ consecutive days.

$$
\begin{gathered}
W^{\text {net }} \approx \sum_{j=1}^{N_{c}} W_{j}^{\text {net }} f_{j}\left(\frac{365}{N_{d}}\right)=\sum_{j=1}^{N_{c}}\left[\sum_{t=t_{1, j}}^{\left[t_{1, j}+24 N_{d}\right.} w_{t}^{* \text { net }}\left(\text { weather }_{t}, P_{t}, \bar{s}_{t}^{\text {avail }}\right)\right] f_{j}\left(\frac{365}{N_{d}}\right) \\
R \approx \sum_{j=1}^{N_{c}}\left[\sum_{t=t_{1, j}}^{t_{1, j}+24 N_{d}} \bar{P}_{t, j} w_{t}^{* \text { net }}\left(\text { weather }_{t}, P_{t}, \bar{s}_{t}^{\text {avail }}\right)\right] f_{j}\left(\frac{365}{N_{d}}\right) \\
f_{j}=\frac{1}{N_{p}} \sum_{i=1}^{N_{p}} \omega_{i, j}
\end{gathered}
$$

The $N_{d}$-day production simulation for the cluster $j$ exemplar begins one day prior to time $t_{1, j}$ and ends one day after the $N_{d}$-day period to minimize end effects. Hourly electricity generation from the limited time-domain simulation $\left(w_{t}^{* \text { net }}\right)$ is differentiated from that provided by the full annual simulation $\left(w_{t}^{\text {net }}\right)$ to denote inconsistencies which may arise due to end effects or the use of cluster-average hourly solar field availability factors $\left(\bar{s}_{t}^{\text {avail }}\right)$. The clustered simulation requires, at most, computation of $24 N_{c}\left(N_{d}+2\right)$ hours, and can be slightly reduced when individual simulations of exemplars which consist of consecutive days are combined into a single simulation to minimize computational overhead and overlap. Electricity generation profiles are computed by dispatching against the electricity price for the exemplar from cluster $j$, while revenue calculations utilize the cluster-average electricity pricing profile $\left(\bar{P}_{t, j}\right)$ defined in equation 3.57. Cluster-average hourly solar field availability factors are computed similarly.

$$
\bar{P}_{t, j}=\frac{\sum_{i=1}^{N_{p}} \omega_{i, j} P_{t, i}}{\sum_{i=1}^{N_{p}} \omega_{i, j}}
$$

\section{Clustering}

Full-year hourly weather and pricing profiles were separated into a set of non-overlapping 2-day groupings, each of which was described by a set of $N_{f}$ features selected from various combinations of: 
1. Average morning and afternoon DNI on each day

2. Average morning and afternoon PPA multiplier on each day

3. Average morning and afternoon difference between actual and clear-sky DNI on each day

4. Average DNI during the day preceding the 2-day grouping

5. Average DNI during the day following the 2-day grouping

All hourly DNI profiles were modified prior to clustering to set $D N I_{t}=0$ in any time period $t$ during which the wind velocity exceeds the maximum allowable value for heliostat operation. As such, excessively windy time periods are presented to the clustering algorithm as if they were cloudy. While only morning/afternoon divisions were considered in this analysis, future efforts may consider time-resolution refinement with a concomitantly larger number of data features, if necessary. Ideally the selected data features will comprise the minimum set necessary to group the data set in a physically meaningful way.

Two clustering algorithms were considered in this study: (1) k-means Arthur and Vassilvitskii, and (2) affinity propagation Frey and Dueck. Each algorithm seeks to group data points so as to minimize the within-cluster sum of squares (WCSS), defined as the sum of the Euclidean distance in $N_{f}$-dimensional space between each data point and the centroid of the cluster to which it belongs. Figure 33 illustrates one example of clustered 2-day DNI profiles created with an affinity propagation algorithm and features 1-4 above.

K-means algorithms are among the most common in data mining applications, and utilize a user-specified number of clusters with unrestricted placement of cluster centroids. Affinity propagation algorithms restrict the cluster centroids to coincide with data points, and form clusters from natural groupings occurring in the data set, with indirect influence over the number of clusters available through specification of the self-affinity of any data point. Each algorithm explicitly assumes hard partitioning, meaning that each data point belongs to one, and only one, cluster $\left(\omega_{i, j}=0\right.$ or 1). However, accumulation of annual electricity generation in this analysis was evaluated with weighting factors $\left(f_{j}\right)$ arising from either hard or soft/fuzzy cluster boundaries. The partition matrix for fuzzy partitioning was computed from equation 3.58, in which $c_{j}$ is the centroid of cluster $j$ and parameter $m=2$ Bezdek, Ehrlich, and Full.

$$
\omega_{i, j}=\left[\sum_{k=1}^{N_{c}}\left(\frac{\left\|x_{i}-c_{j}\right\|}{\left\|x_{i}-c_{k}\right\|}\right)^{\frac{2}{m-1}}\right]^{-1}
$$

\section{Comparison with full annual simulation}

Annual electricity generation, revenue, and overall cash flow estimated from reduced simulations based on clustered profiles were compared with the corresponding values from a full annual simulation. These comparisons were carried out using three different sets of metrics, k-means and affinity propagation clustering algorithms with either hard and soft cluster weighting, and two sets of independent variable values: (1) independent variation of each variable at low/mid/high levels or (2) variable combinations based on Latin hypercube sampling within the full parameter space. Figure 34 illustrates example results for annual revenue units and the overall cash flow objective using 25 randomly selected independent variable sets, TMY weather data for Daggett, CA, the SAM default block PPA multiplier schedule, 2-day groupings, and data features 1-4 above. Figure 35 illustrates the error in cash flow relative to the full annual simulation for each variable combination and clustering algorithm, and Figure 36 quantifies the simulation time relative to the full annual simulation for each variable combination. Note that the total simulation time in Figure 36 includes the time required to generate the heliostat field layout and run the current degradation, soiling, and availability models, neither or which is altered by the clustered simulation methodology considered here.

Figure 34 suggests that reduced simulations based on clustered profiles can closely capture overall trends in electricity generation, revenue, and cash flow, even with a relatively small number of clusters. The average (absolute) error across all variable combinations in Figure 35 is approximately $\$ 22 \mathrm{M}$ and $\$ 17 \mathrm{M}$ for k-means and affinity propagation algorithms, respectively, at 25-30 clusters, decreasing to $\$ 16 \mathrm{M}$ and $\$ 10 \mathrm{M}$ at 60-65 clusters. This represents at most $2 \%$ of the highest cash flow observed from this set of variable values $(\$ 1139 \mathrm{M})$ for even small numbers of clusters.

92

This report is available at no cost from the National Renewable Energy Laboratory (NREL) at www.nrel.gov/publications 

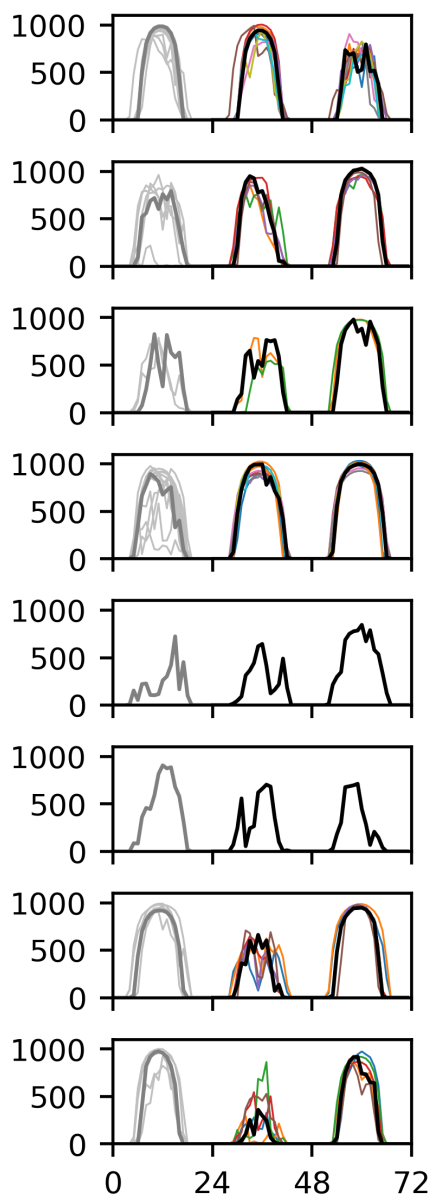
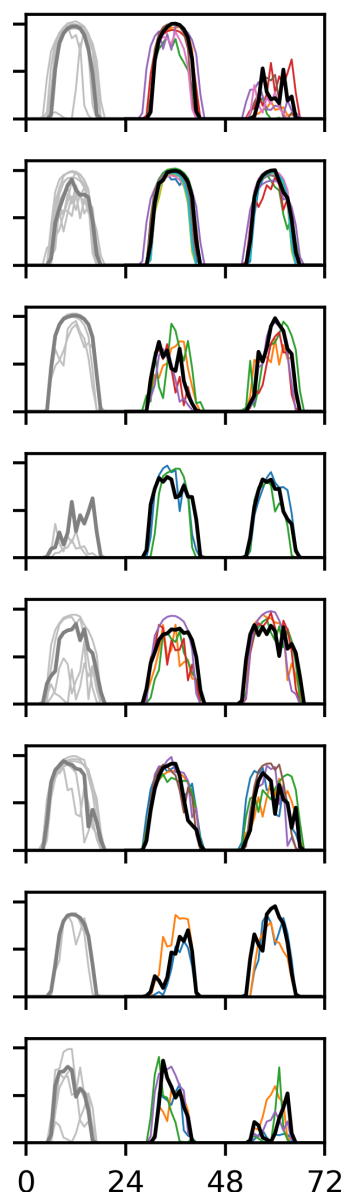
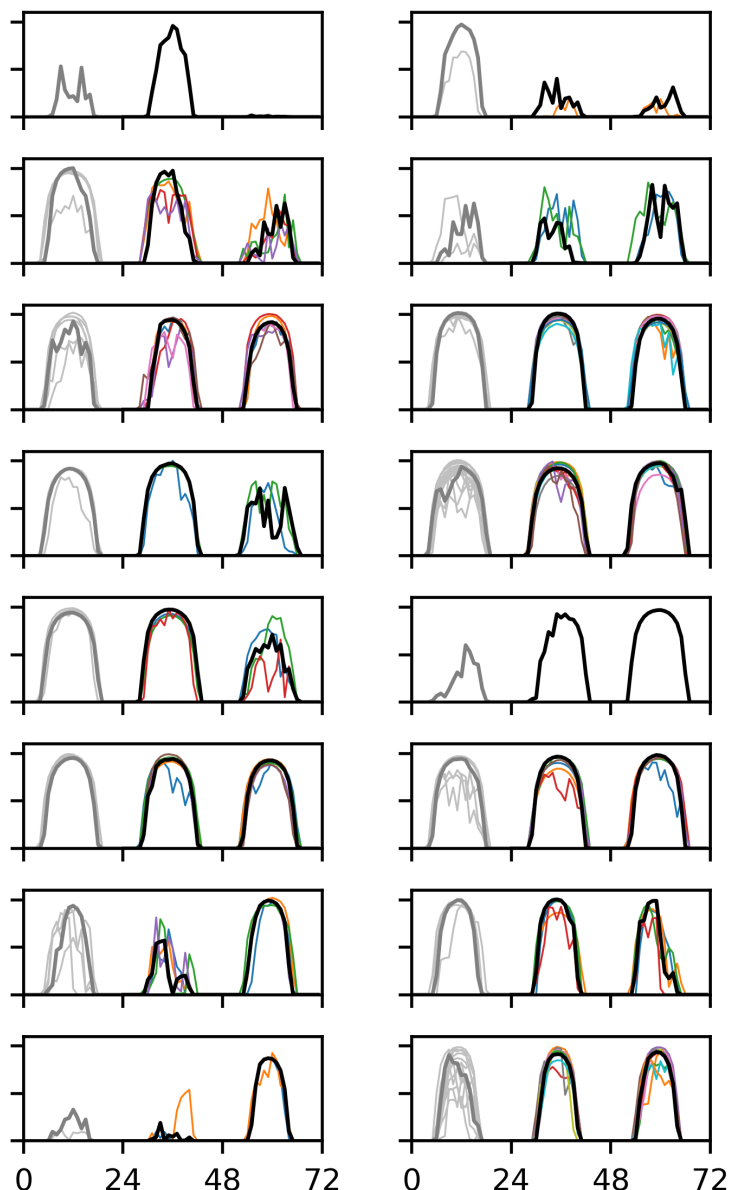

Figure 33. Clustered 2-day DNI profiles (including previous day). Bold lines indicate the cluster exemplar profile

Simulations with 60-65 clusters reproduced the full annual simulation results within approximately $1-1.5 \%$ of the highest cash flow (on average), and still provide a computational cost savings on the order of 25-50\%. Comparisons of full annual vs. clustered simulations have also been completed for: (1) Tonopah, NV TMY weather data with the SAM default block PPA multiplier schedule, and (2) Rice, CA 2015 weather data with CAISO 2015 hourly LMPs at a nearby node. These simulations exhibit a similar error magnitude and are omitted here for brevity. In most cases clusters formed with the affinity propagation algorithm slightly outperform those created with the k-means algorithm, likely owing to the constrained selection of a cluster centroid from the data set. No consistent preference was observed for hard vs. soft partition weighting.

Next, we extend the algorithms to optionally consider multiple years of weather and electricity pricing data simultaneously. This extension is motivated by the observation that multiple years of weather data may not contain a substantially larger number of characteristic repeating weather/pricing patterns than are observed within a single year. Thus, for example, if 30 exemplar profiles are needed to adequately capture performance in a single year, it may be possible that only 40 exemplar profiles are required to adequately capture performance over the course of 10 years.

Here, we follow an analogous procedure to the previous analysis, but expand the data set to consist of all two-day blocks which occur throughout the duration of multiple years. Thus any given cluster may contain two-day blocks 

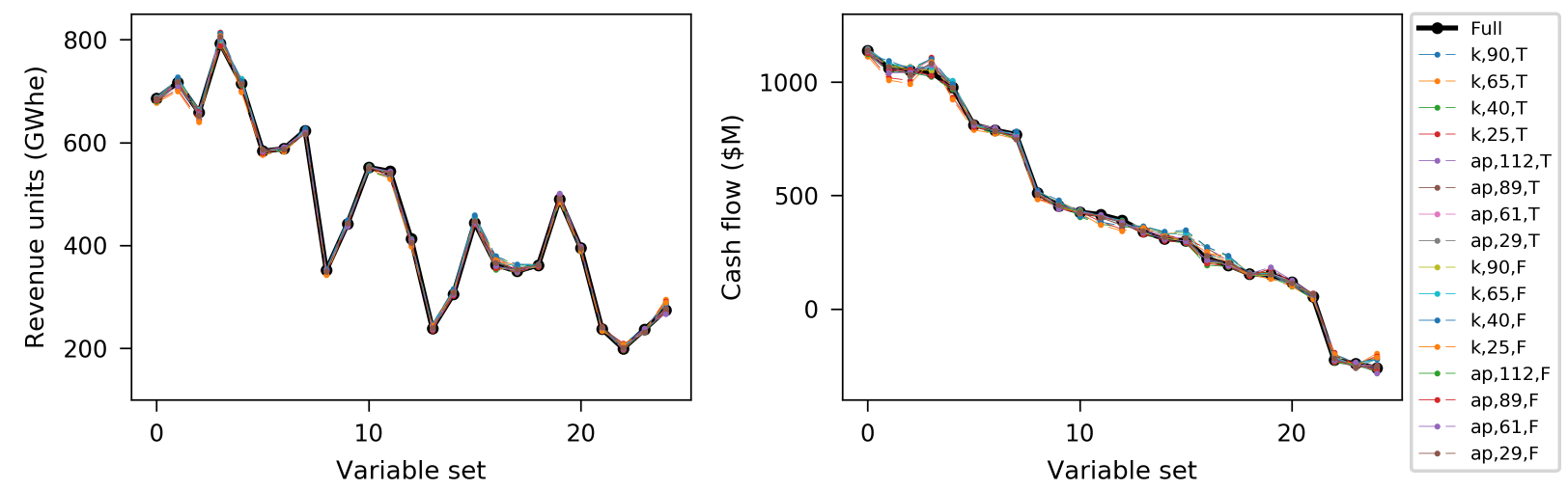

Figure 34. Comparison of full and clustered simulations at 25 randomly selected independent variable combinations (displayed in order of decreasing cash flow).
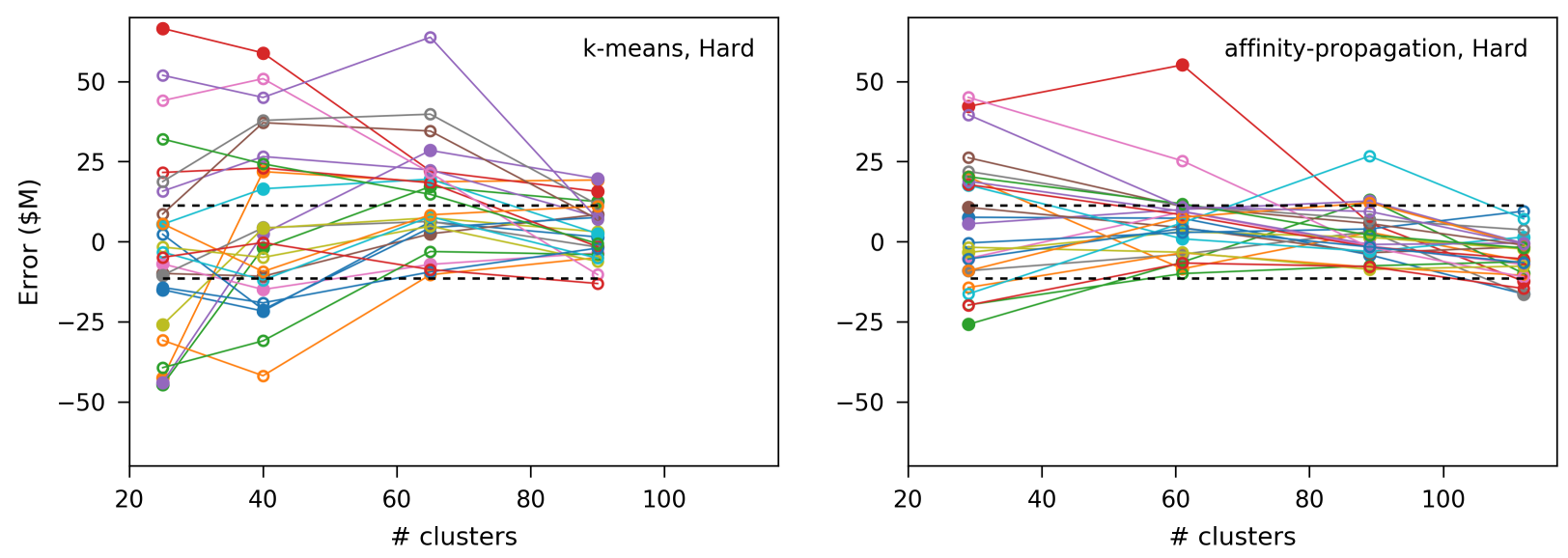

Figure 35. Cash flow error from clustered simulations at 25 randomly selected independent variable combinations. Dashed black lines represent $1 \%$ of the highest cash flow from the set of full simulations.

from different years, and cluster exemplar profiles can be pulled from any of included years. After simulation of the cluster exemplars, electricity generation profiles and, correspondingly, total generation and revenue in any given year can be re-created by stitching together the appropriate set of simulated exemplar profiles. This approach, if successful, can provide an advantage over simply using TMY weather data by retaining information pertaining to year-to-year variability in production or revenue which may be of concern for CSP developers.

In the following results we consider two combinations of weather/pricing data: (1) Daggett, CA 2005-2015 weather data with the SAM default block electricity pricing schedule, and (2) Rice, CA 2005-2015 weather data with actual CAISO 2015 hourly locational marginal prices (LMPs). In each case we apply the same pricing profile to all years for simplicity, but the algorithms are generalized to permit application of different price profiles for each year. Figures 37 and 38 illustrate the yearly revenue for a CSP plant with a solar multiple of three and 15 hours of thermal storage. Annual revenue units are defined in equation 3.59, in which $w_{t, y}^{n e t}$ and $P_{t, y}$ are, respectively, the net electricity production and electricity price at time point $t$ during year $y$, and $\overline{P_{y}}$ is the yearly average electricity price in year $y$. As identical hourly electricity prices are applied to all years, the year-to-year variability in Figures 37 and 38 arises only from variability in weather. 


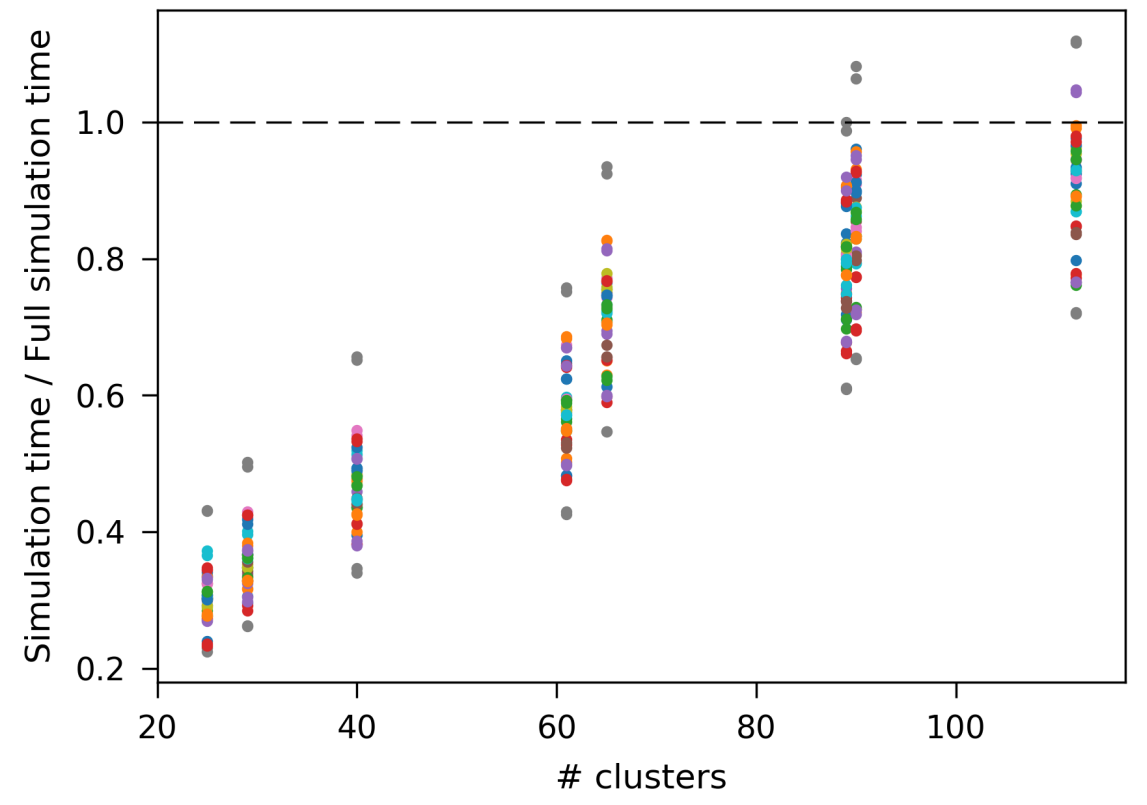

Figure 36. Simulation time relative to full annual simulation.

$$
R_{y}=\sum_{t=1}^{8760} w_{t, y}^{n e t}\left(\frac{P_{t, y}}{\overline{P_{y}}}\right)
$$

The individual year PPA price in Figures 37 and 38 represents the value that would be calculated based only on the revenue from that year. Dashed lines indicate both the average values over the 11 year period, and the values calculated using TMY weather data. Black lines illustrate values calculated by running the full simulation for each of the 11 years, whereas colored lines illustrate values calculated by running simulations for only the designated number of cluster exemplars.
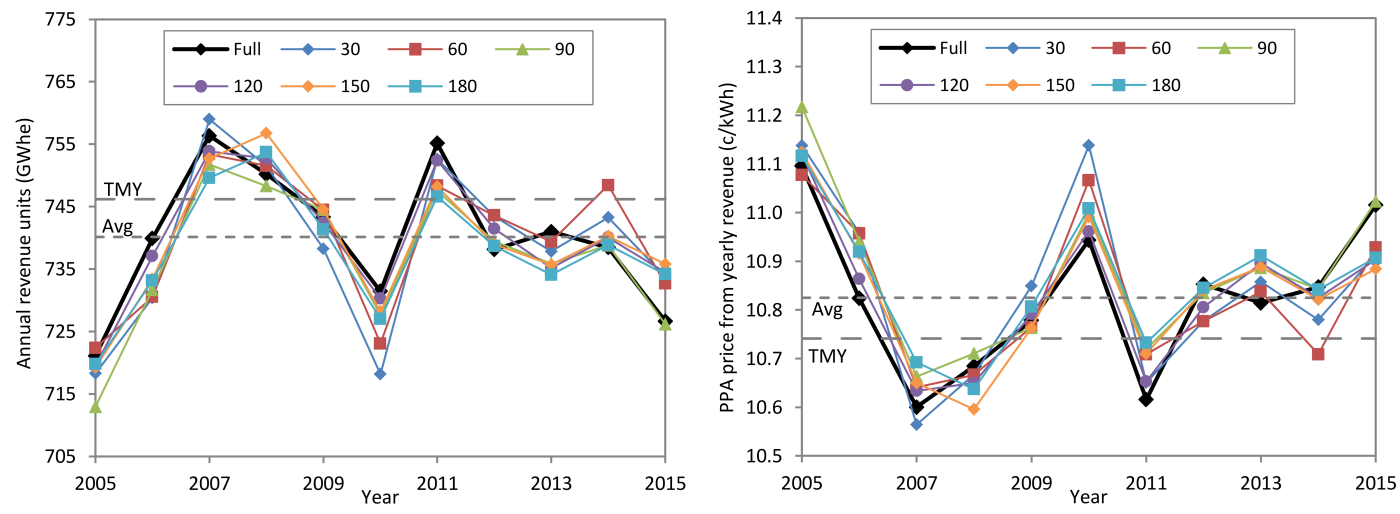

Figure 37. Yearly performance for Daggett, CA with a block electricity price schedule

Cluster-based simulations require between 7\% (30 clusters) and 30\% (180 clusters) of the time required to run full simulations for all 11 years. As expected, values based on TMY weather data capture the 11-year average closely 

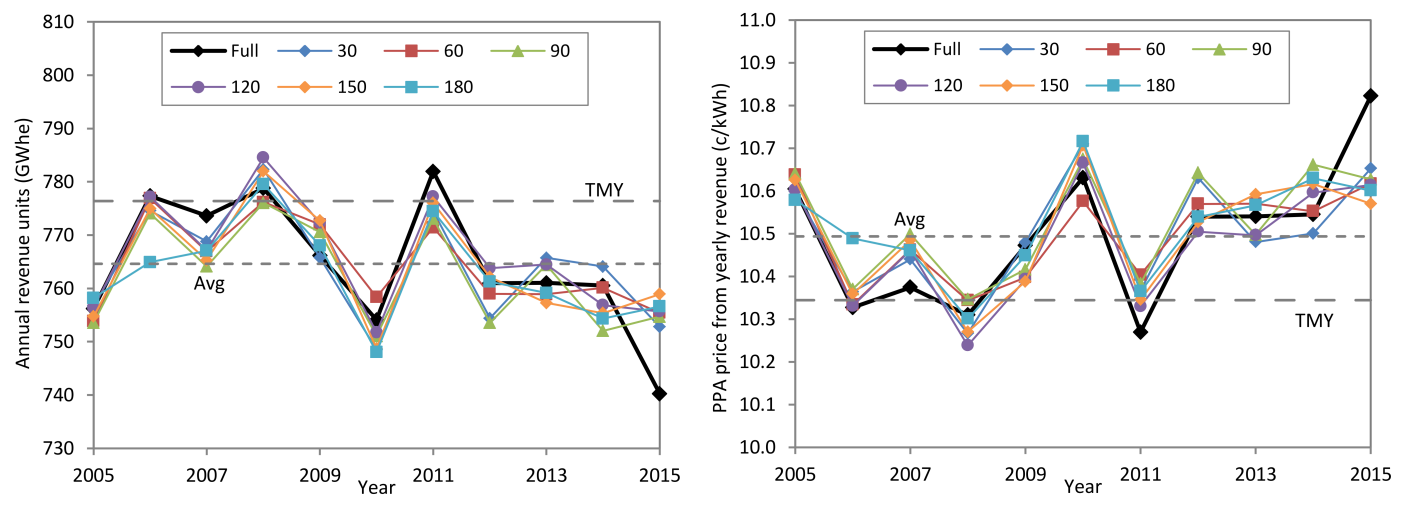

Figure 38. Yearly performance for Rice, CA with CAISO locational marginal prices from 2015

(within approximately $0.8 \%$ and $1.5 \%$ for the Daggett and Rice cases, respectively). Yearly variability is approximately $\pm 2.5 \%$ of the multi-year average for each case. The cluster-based simulations show minor deviations in certain single years, but capture the general year-to-year trends with even a small number of clusters. Root-mean-square errors over the 11-year period for the cluster-based simulations relative to the full simulation are approximately $0.7-0.8 \%$.

The data clustering algorithm's implementation is integrated with the rest of the DAO-Tk package, and may be used directly by setting the variables is_use_clusters and n_clusters to 'true' and some positive integer, respectively; a minimum of 25 clusters is recommended, given the testing experience described above. The number of days evaluated per cluster may be adjusted via the variable cluster_ndays, and the number of days in the plant warm-up period for each cluster is updated by setting the variable cluster_nprev.

\subsubsection{Multithreading}

The data clustering procedure described in Section 3.5.1.1 allow for the dispatch optimization problem from Section 3.4 to be subdivided into separate subproblem that we may solve in parallel. Further, the cycle availability model in Section 3.3.5 includes an option to run multiple replicates in order to obtain summary statistics on estimated availability and revenue losses due to the failure of components in the power cycle. To exploit the structure of these two models, we have implemented a multithreading implementation in which the each thread may be assigned a separate subproblem or replicate. This reduces solution time by a factor that depends on the number of computing cores available, dispatch subproblems, and cycle model replicates.

The maximum number of threads available for use is denoted in DAO-Tk by the variable n_sim_threads; this must be at least two in order to leverage the multithreading approach. Note that this is only used for clustering if clustering is used and the data clusters are not connected, i.e., is_use_clusters $=1$, n_clusters $>1$, and is_run_continuous $=0$. Similarly, multithreading is used for the cycle availability model only if more than one replicate is selected for the cycle availability model, i.e., num_cycle_scenarios $>1$.

\subsubsection{Stochastic seeding}

The simulations we describe in Section 3.3.3, 3.3.4, and 3.3.5 utilize the WELL512 procedure developed in Panneton, L'Ecuyer, and Matsumoto to obtain uniform $[0,1]$ realizations. These, in turn, are used to obtain realizations of the component lifetimes, repair times, and degradation measures for these models using methods including, but not limited to, evaluating the inverse of the underlying cumulative distribution function at the uniform random variate. By using common random number streams for each design that is evaluated in the outer-loop optimization described in Section 3.2, we induce positive correlations that allow for comparison between designs using fewer replicates than we would by using independent sampling. 
Custom seeds may be used for the solar field availability and optical models by setting the variables avail_seed and degr_seed, respectively, to specific integer values.

\subsubsection{Uncertainty heuristics}

Section 3.4.2 details the procedure we use to incorporate uncertainty into our optimization model. This method, which proposes an incentive for thermal energy storage at the end of a subproblem's time horizon according to future pricing forecasts, incorporates much of the uncertainty that a full stochastic program would cover without the computational burden of solving such a model. For more details on the method and its relative savings in computing time, see Section 3.4.2.

\subsubsection{Open-source code}

The DAO-Tk project has been released as open-source software (OSS). This accomplishes several things:

- It allows users full access to the code and algorithms, improving likelihood of adoption

- It keeps the project up to date as progress is made

- It extends the life of the project beyond the current window for DOE funding

- It enables users to customize the software for their application or plant configuration

- It is an effective mechanism for providing research transparency

Github.com is the platform that hosts the source code for dao-tk. NREL maintains a dedicated channel and membership at https://github.com/NREL, where many other NREL open-source projects are available, including SAM, SolarPILOT, SolTrace, OpenStudio, and others. The Github platform uses the Git version control system to track changes, issues, versions, and to provide documentation. The Github page and repository is located at https://github.com/NREL/dao-tk, and the main Readme file documents the project background and goals, as well as a brief description of the submodels within dao-tk and installation instructions. Additionally, multiple sub-models include separate Readme files that offer further details, such as submodel inputs and outputs.

\subsubsection{Application interface}

Here, we provide an introduction to the user interface. While this may serve as a guide to the user for an overview regarding the functionality of the program, we refer the reader to the example scripts included with the DAO-Tk release as an additional reference. The model descriptions in Sections 3.1-3.4 provide further details on each submodel within the application.

\subsubsection{User interface overview}

The program contains three main windows: (i) the scripting window where users can draft and revise their script, access documentation, and load or save script files, (ii) the data window where input and output data objects are presented in tabular format, and can be plotted using time-series graphing capabilities, and (iii) a log window that records messages and runtime information generated by the simulations. Figure 39 displays an overview of the UI layout, which includes labels to denote the three windows and other key features in the program. There are two main file types that are accessed in the main window. The first, project files, contain settings related to the system, simulated data, collection of scripts, and solver settings. Projects may be newly generated, opened or saved via the UI by selecting the button labeled DTK at the top-left corner of the window. The second file type, script files, may be added to project files, and also conduct model evaluation procedures. 


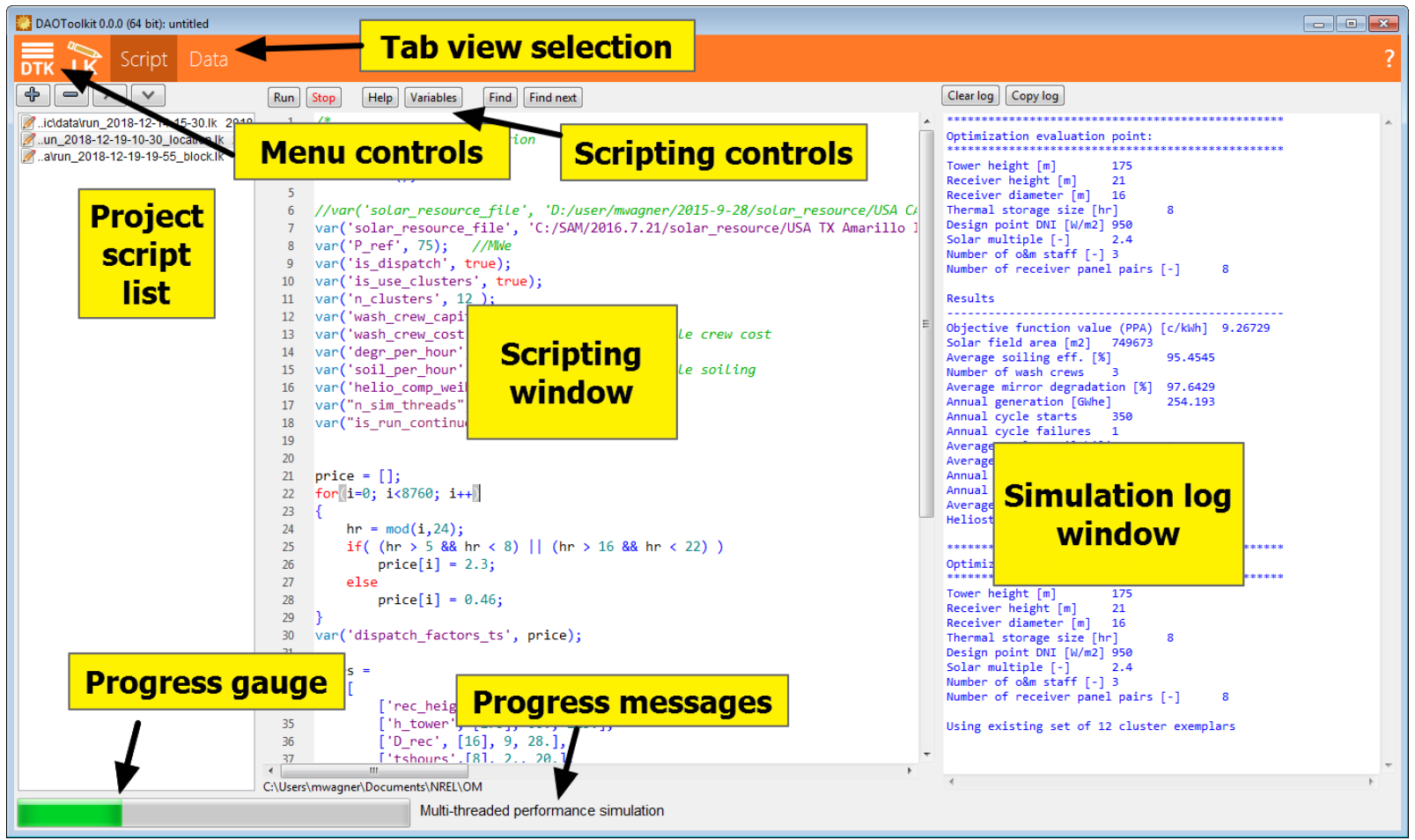

Figure 39. Screenshot of the application's main UI window; labels describe the different components of the layout

\subsubsection{Model variables}

Figure 40 displays the program's variable dialog, which is accessed by selecting the button labeled Variables in the main window. The script commands that set and return a variable's value are shown in 41; these may be entered directly into the scripting window, but are also automatically generated by the Insert SET and Insert GET links under each variable in the dialog, respectively. The variables are populated with default value and bounds in the event that no guidance is provided in the script.

Multiple variables may be adjusted manually in table form, including lower and upper bounds by assigning a table of inputs to the model parameter ovars with an example as follows:

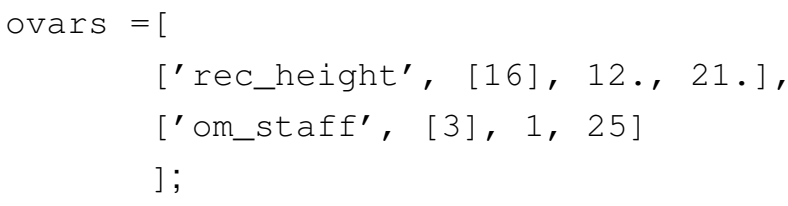

Each variable-specific entry in the table takes the form

$$
[\text { 'name', }[\mathrm{val}], 1 \mathrm{~b}, \mathrm{ub}]
$$

in which name, val, lb, ub denote the variable's name, initial value, lower bound, and upper bound, respectively. 


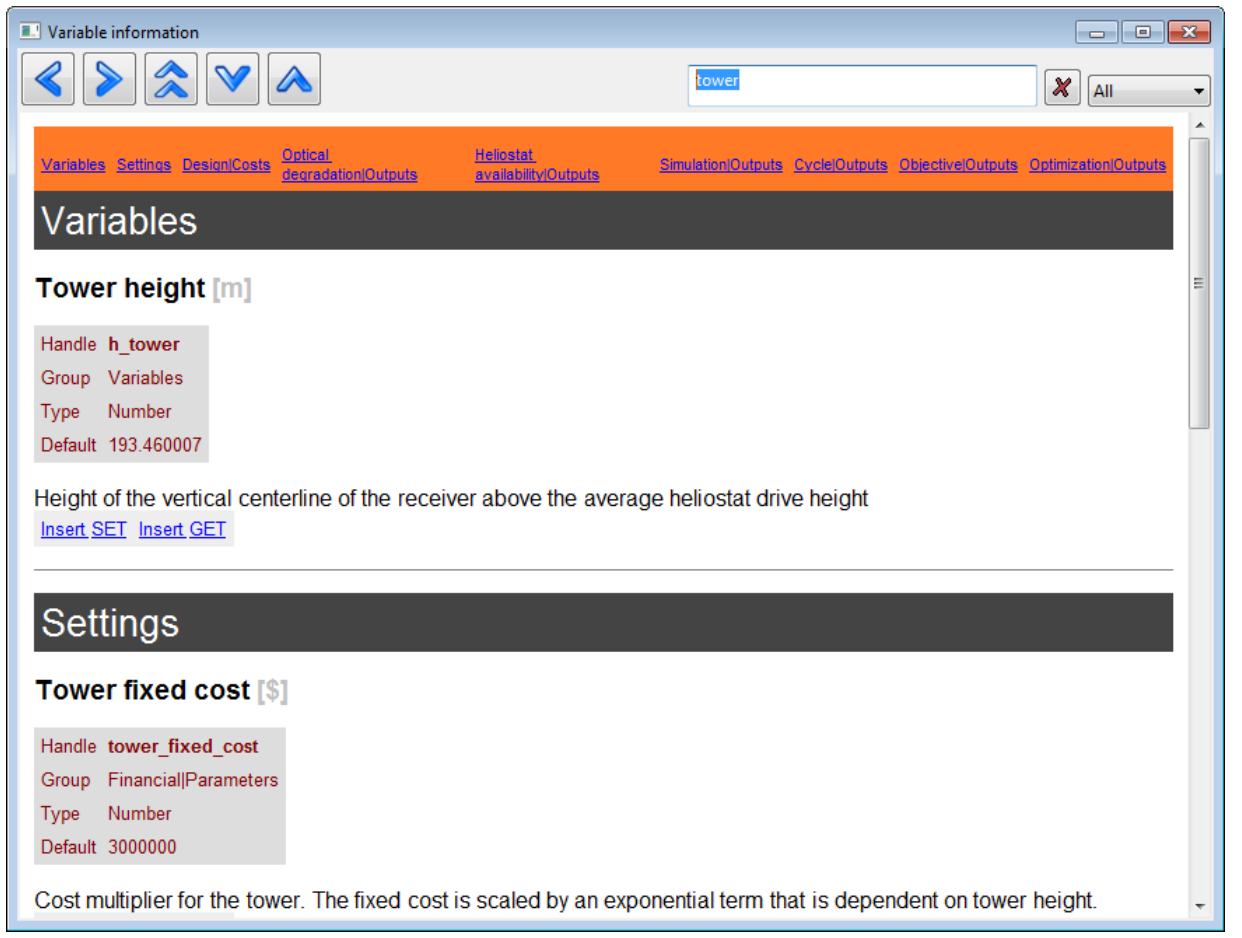

Figure 40. Screenshot of the application's variable dialog, which is accessible by selecting the button labeled Variables in the scripting window 


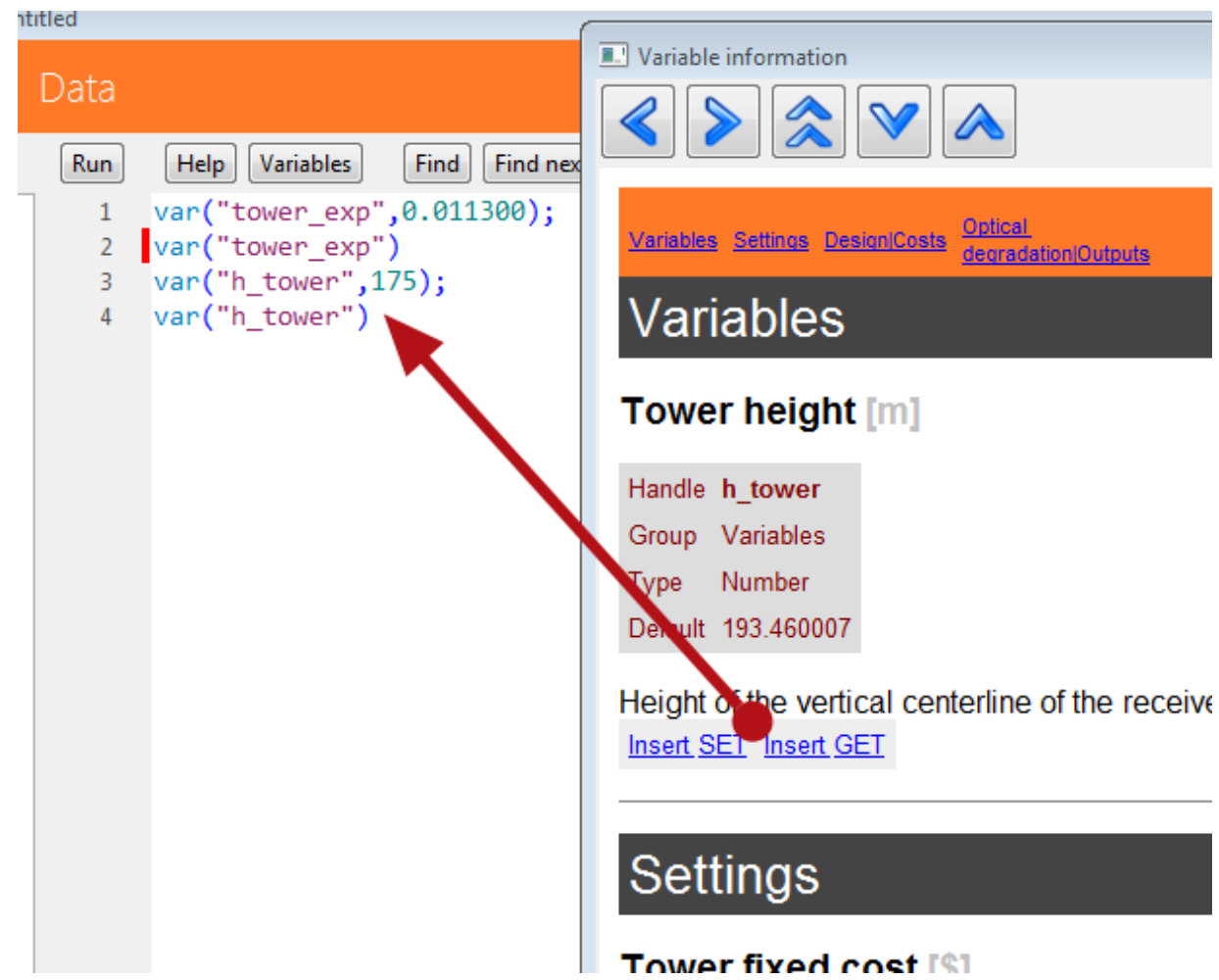

Figure 41. Screenshot of the application's scripting window, with commands used to set and get the variables tower_exp and $h$ _tower; these commands are automatically generated by selecting the Insert SET and Insert GET links in the variable dialog to set and return each variable's value, respectively 


\subsubsection{Model settings and parameters}

Model settings and parameters that serve as input to the solution method and all sub-models are located below the variables within the variable dialog. This section can be reached by scrolling to the end of the variables, or by selecting the link titled "Settings" at the top of the variable dialog.

Similar to the variables, these settings may be adjusted manually in the script window using the command

$$
\operatorname{var}(\text { "name", val), }
$$

in which name and val denote the setting or parameter name and the desired value, respectively; similarly, the command var ("name") returns the current value of the parameter. Alternatively, there are analogous Insert SET and Insert GET links for each variable which will generate these commands automatically. Unlike variables, parameters in the model only accept fixed values, and do not include upper or lower bounds.

\subsubsection{Functions}

A reference to all the function names and descriptions are available in the help dialog, which is accessed by selecting the button labeled Help in the main window, as shown in Figure 42. Many of the functions that include inputs in the form of an options table; if no options are included as arguments in the function call, the current model settings (including all commands up to that part of the script) will be used.

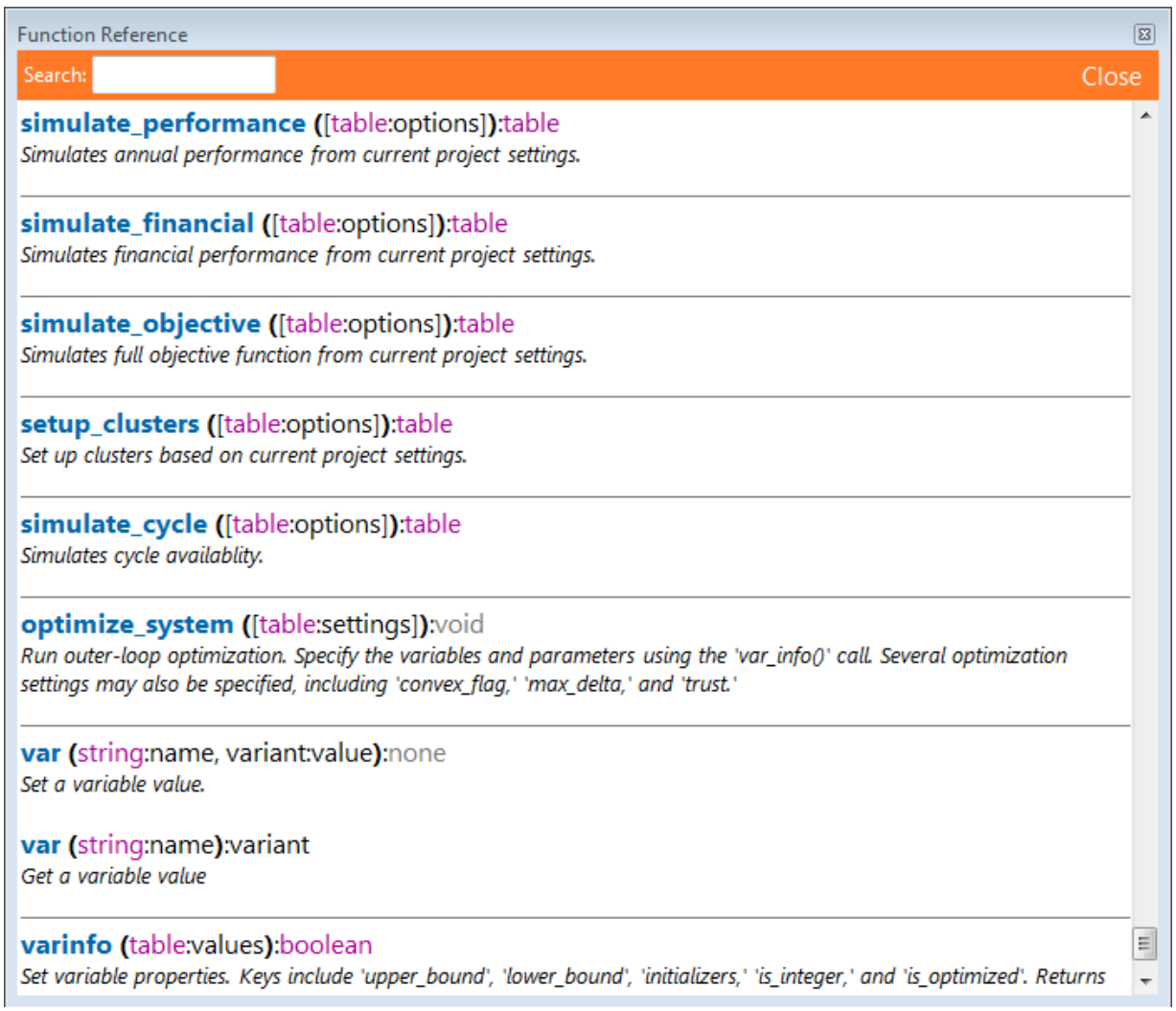

Figure 42. Screenshot of the program's function reference, which is accessed by selecting Help in the main window

\subsubsection{Outputs and Visualization}

The Data tab provides a complete listing of all the input and output data within the program. The window on the left side of the data tab includes expandable collections of model settings, sub-model inputs, and model outputs. The 
search bar located near the top of the Data tab allows the user to search for specific inputs and outputs, and checking the box of a desired parameter will display its value(s) on the right-hand side of the tab. Any previously selected parameters that do not fit the search criteria will remain in view on the data tab. Figure 43 displays an example of using the search bar to select and view a time-series of net power generation as output, after the price signal time series was already selected.

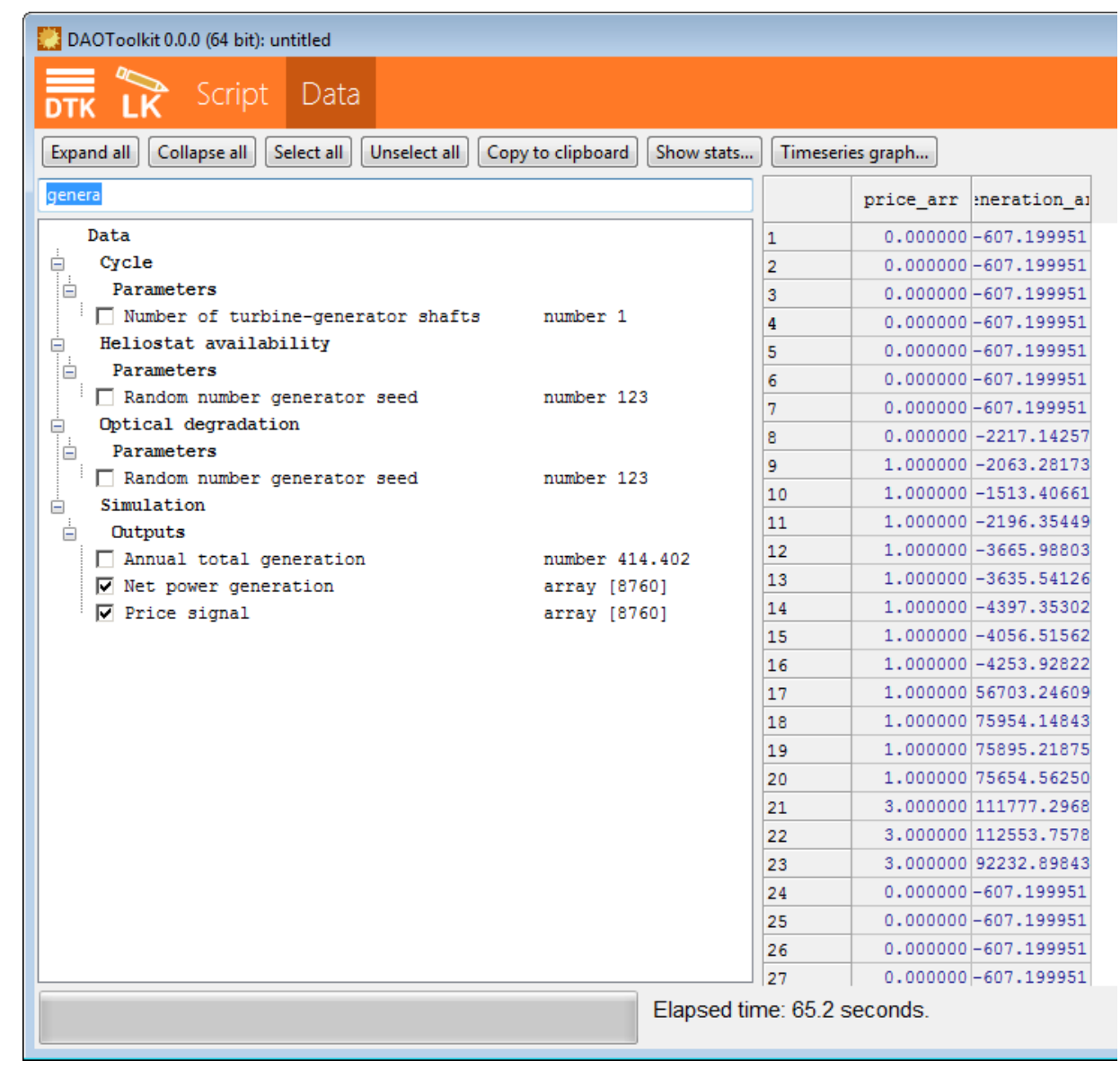

Figure 43. Screenshot of the Data tab, in which the search bar is used to select and view a time-series of net power generation in the right-hand side of the window; previously selected outputs that do not fit the search criterion (i.e., price signal) remain in view

Summary statistics and various visualizations of time-series parameters are available within the Data tab. After selecting the desired parameters by checking the appropriate boxes as shown in Figure 43, selecting the Show stats... button loads the Variable data window, which displays a collection of aggregate and time-varying summary statistics for given outputs, as shown in Figure 44.

Selecting the Timeseries graph... button displays the Timeseries viewer window, which provides the user with a collection of options for data visualization: Figures $44,45,46$, and 47 display a table of summary statistics, timeseries plot, monthly profile, and heat map of net power generation, respectively; Figure 45 includes the price signal, using a common horizontal axis to denote the time of year. 


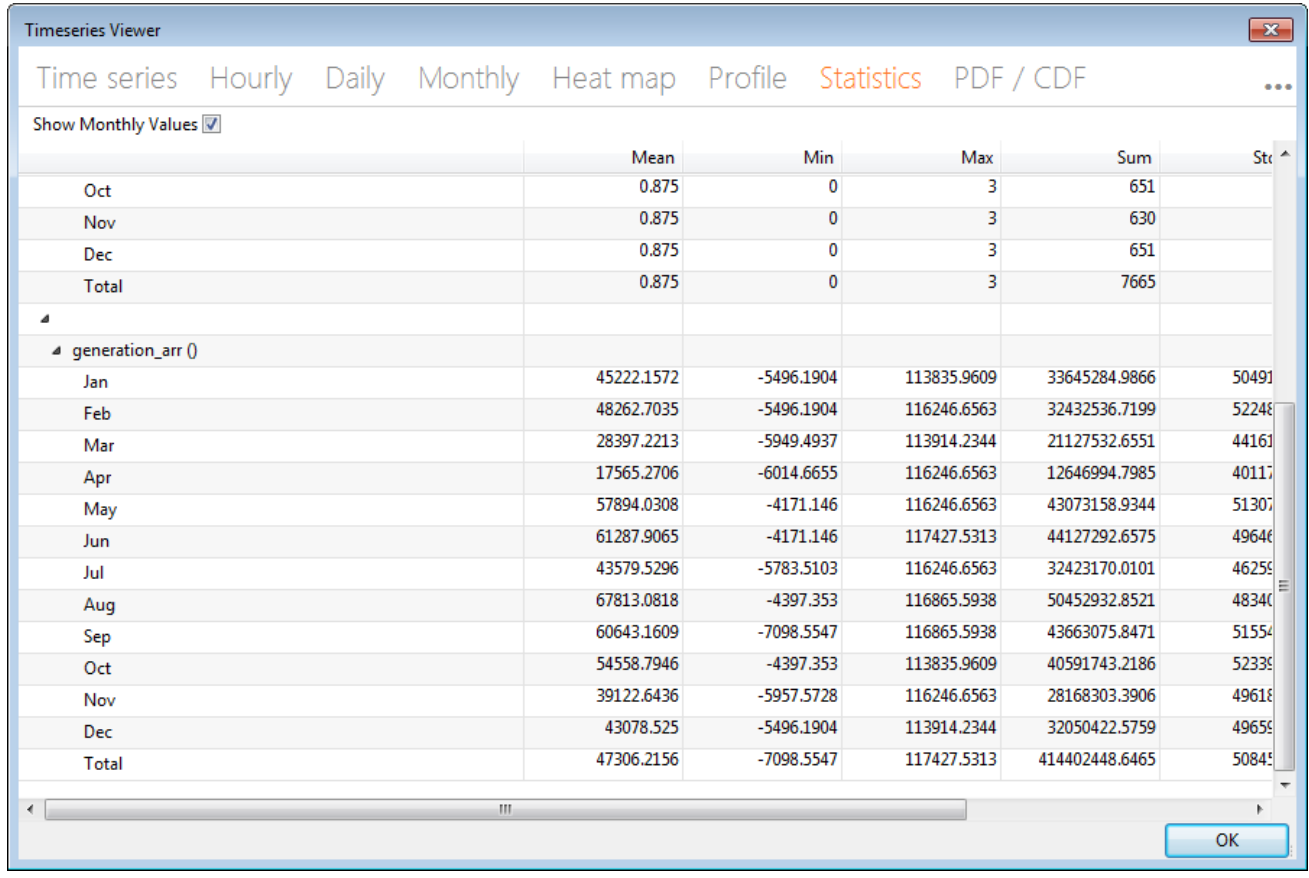

Figure 44. Screenshot of the Timeseries viewer window displaying a table of summary statistics for net power generation

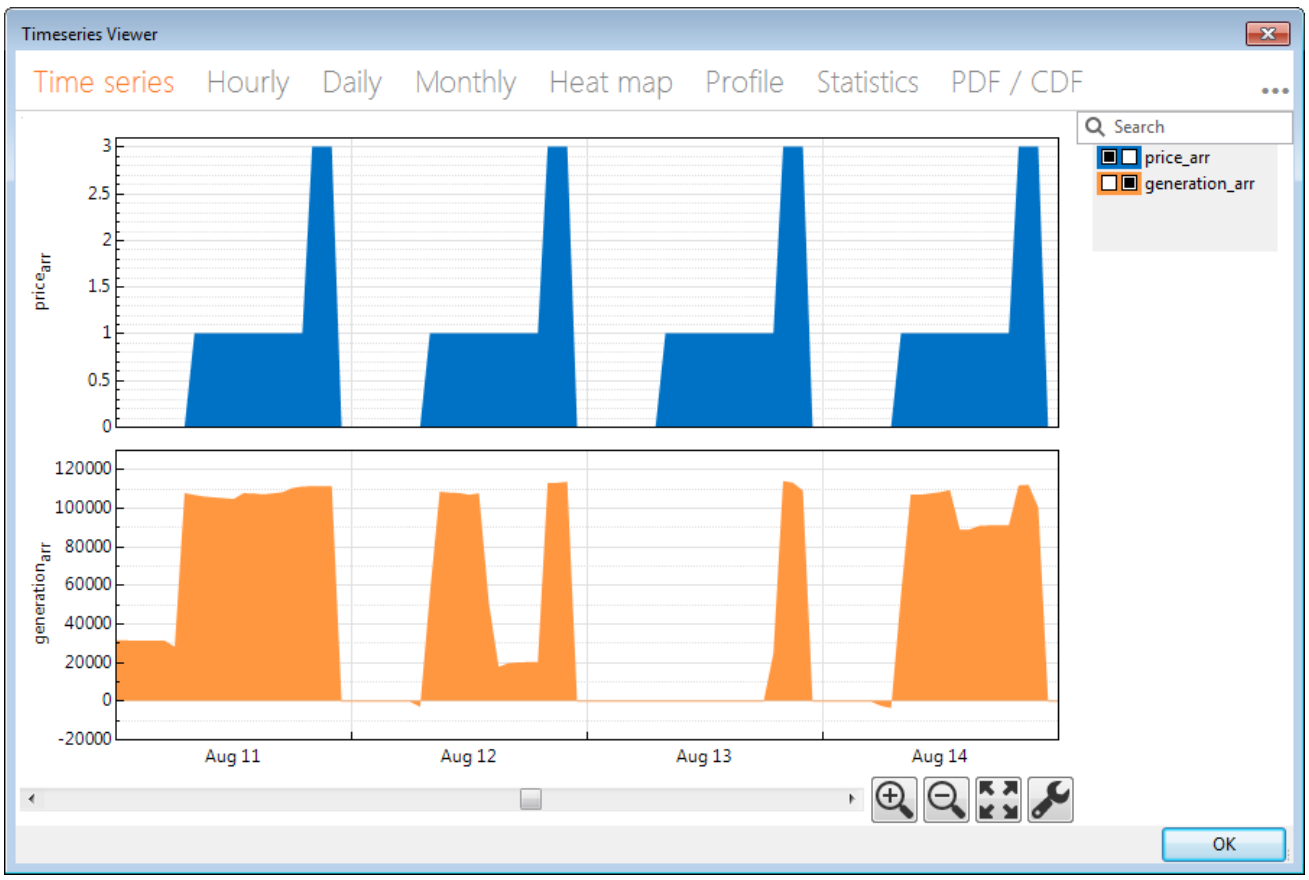

Figure 45. Screenshot of the Timeseries viewer window displaying a time-series plot of the price signal and net power generation 


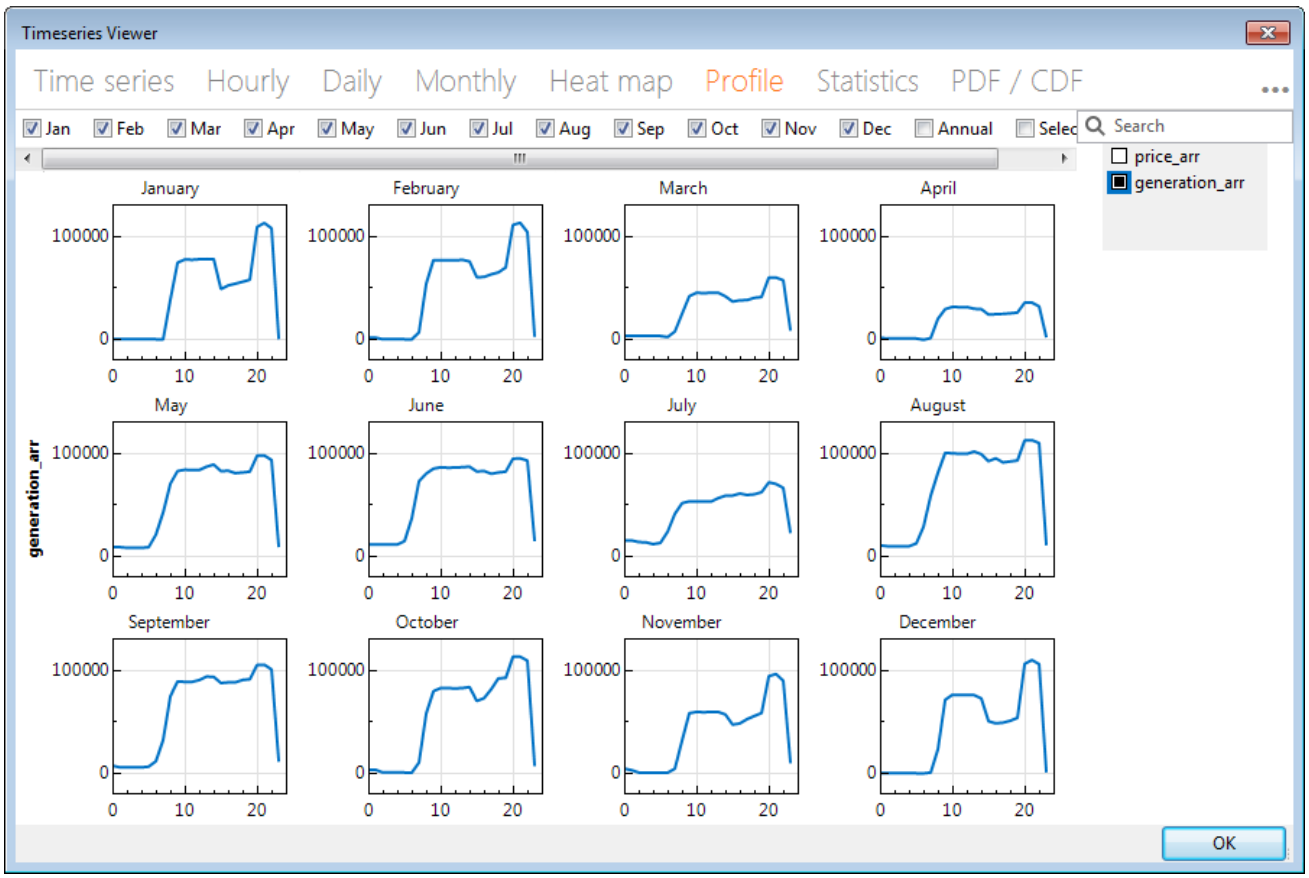

Figure 46. Screenshot of the Timeseries viewer window displaying a monthly profile of net power generation

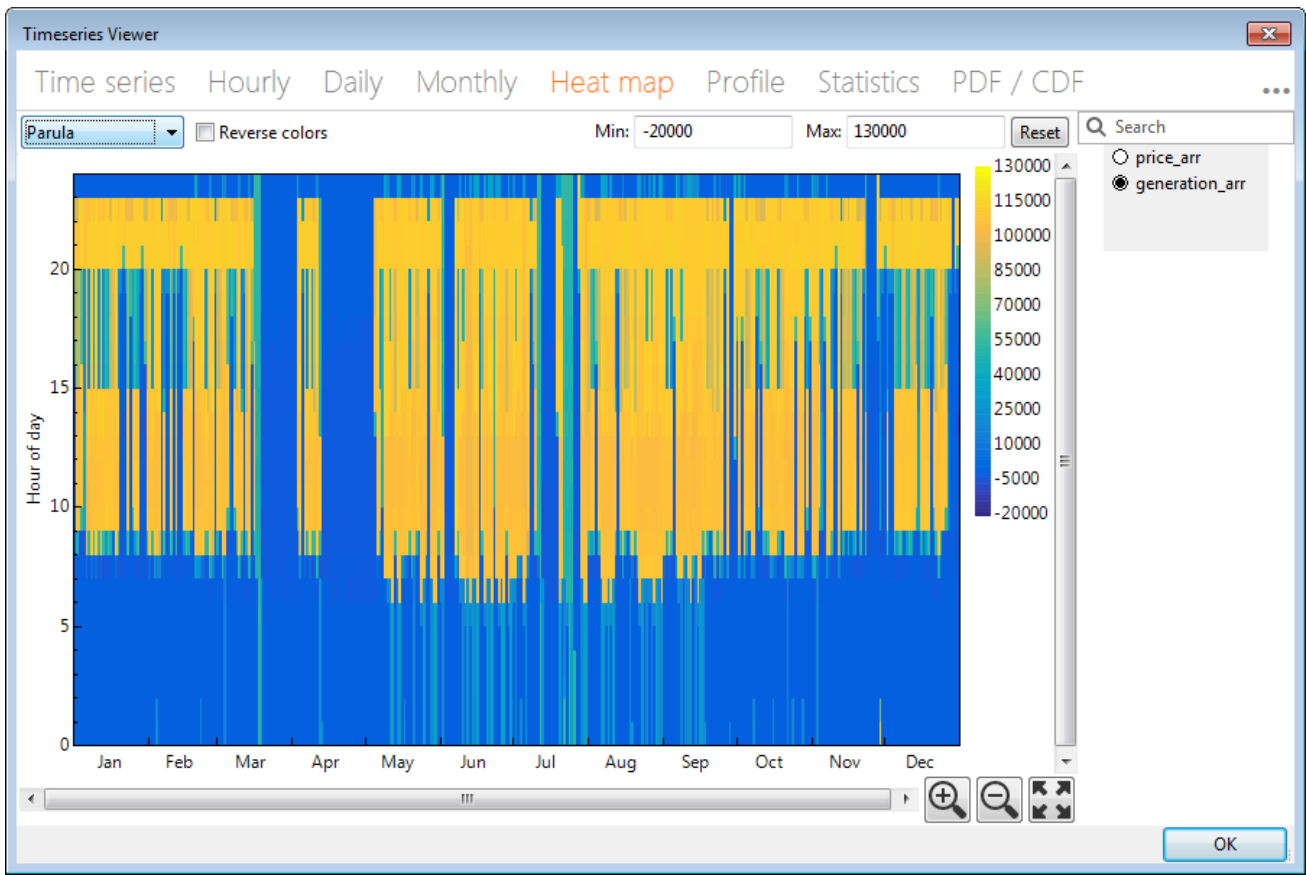

Figure 47. Screenshot of the Timeseries viewer window displaying a heat map of net power generation 


\subsubsection{Overall system design and operation}

In work leading up to this project, the subject of optimization was system-level design variables such as solar field and receiver size, thermal storage capacity, etc. Since the completion of that project at the end of the last calendar year, several developments have made possible additional progress in system optimization in parallel with current efforts on real-time optimization. These include, for example, elimination of the O\&M staff and washing crews integer variables by solving for their values within dedicated subproblems, improved data visualization and reporting, and several bug fixes in the software. Consequently, we have been able to undertake several case studies looking at the impact of certain parameters on optimal design values and on cost-of-energy metrics like LCOE and PPA price. The results of these case studies are briefly presented here.

Prior work suggested complex interactions and relationships between design variables and parameters such as soiling rate, cost of equipment, O\&M costs, etc. To better quantify these relationships, we consider the design optimization of a system under varying assumptions for (a) power cycle efficiency, (b) heliostat mean-time-to-failure (MTTF), (c) heliostat specific cost, and (d) heliostat soiling rate. Table 29 summarizes system assumptions and design parameters that are used for each case study.

The approach taken uses scripts written for DAO-Tk to optimize the six design variables in Table 30 within the allowable $\min / \max$ range and using the specified nominal value as the initial guess. For each parameter value among those specified in Table 30, the software was allowed to find the variable values that minimize the PPA price. Parameters were varied one-at-a-time with other parameters reset to their assumed or default value. In all, six levels of power cycle efficiency, five levels of heliostat MTTF and specific cost, and four values of heliostat soiling rate were considered.

\section{Table 29. Key parameters used for the case studies}

\begin{tabular}{l|c}
\hline Item & Value \\
\hline Gross power rating $(\mathrm{MW})$ & 105 \\
\hline Is dispatch optimized? & Yes \\
Number of simulation clusters & 24 \\
Daytime pricing multiplier (day hours 8-19) & 0.75 \\
Peak pricing multiplier (day hours 20-22) & 2.25 \\
Off-peak pricing multiplier & 0 \\
Location & Daggett, CA (TM3) \\
Convergence tolerance & 0.001
\end{tabular}

Table 30. Optimization variables solved for each case and parameters that are varied for each case study.

\begin{tabular}{|l|cccc|}
\hline Item & Units & Nominal & Min & Max \\
Variables & & & & \\
\hline Receiver height & $\mathrm{m}$ & 18 & 9 & 25 \\
Tower height & $\mathrm{m}$ & 180 & 60 & 225 \\
Receiver diameter & $\mathrm{m}$ & 18 & 9 & 28 \\
Thermal storage size & $\mathrm{hr}$ & 8 & 2 & 20 \\
\hline $\begin{array}{l}\text { Design-point DNI } \\
\text { Solar multiple }\end{array}$ & $\mathrm{W} / \mathrm{m}^{2}$ & 950 & 600 & 1100 \\
\hline Parameters & & 2.5 & 0.8 & 5 \\
\hline Power cycle efficiency & - & 0.412 & 0.34 & 0.54 \\
Heliostat mean time to failure & $\mathrm{hr}$ & 15000 & 5000 & 40000 \\
Heliostat specific cost & $\$ / \mathrm{m}^{2}$ & 145 & 50 & 150 \\
Heliostat soiling rate & $\% /$ week & 2.5 & 1 & 25 \\
\hline
\end{tabular}

Figure 48 shows the optimal variable values and corresponding metrics of interest for the power cycle efficiency study. Each subplot shows the starting value (the default variable value, or the calculated metric at the first iteration) 
on the black line, and shows the optimized value or metric on the green line. The difference between the black and green points represents the change or improvement made during optimization at each parameter value. Also shown in dotted blue is the converged value, which may differ slightly from the best point found during optimization.

Several important observations can be made from this analysis:
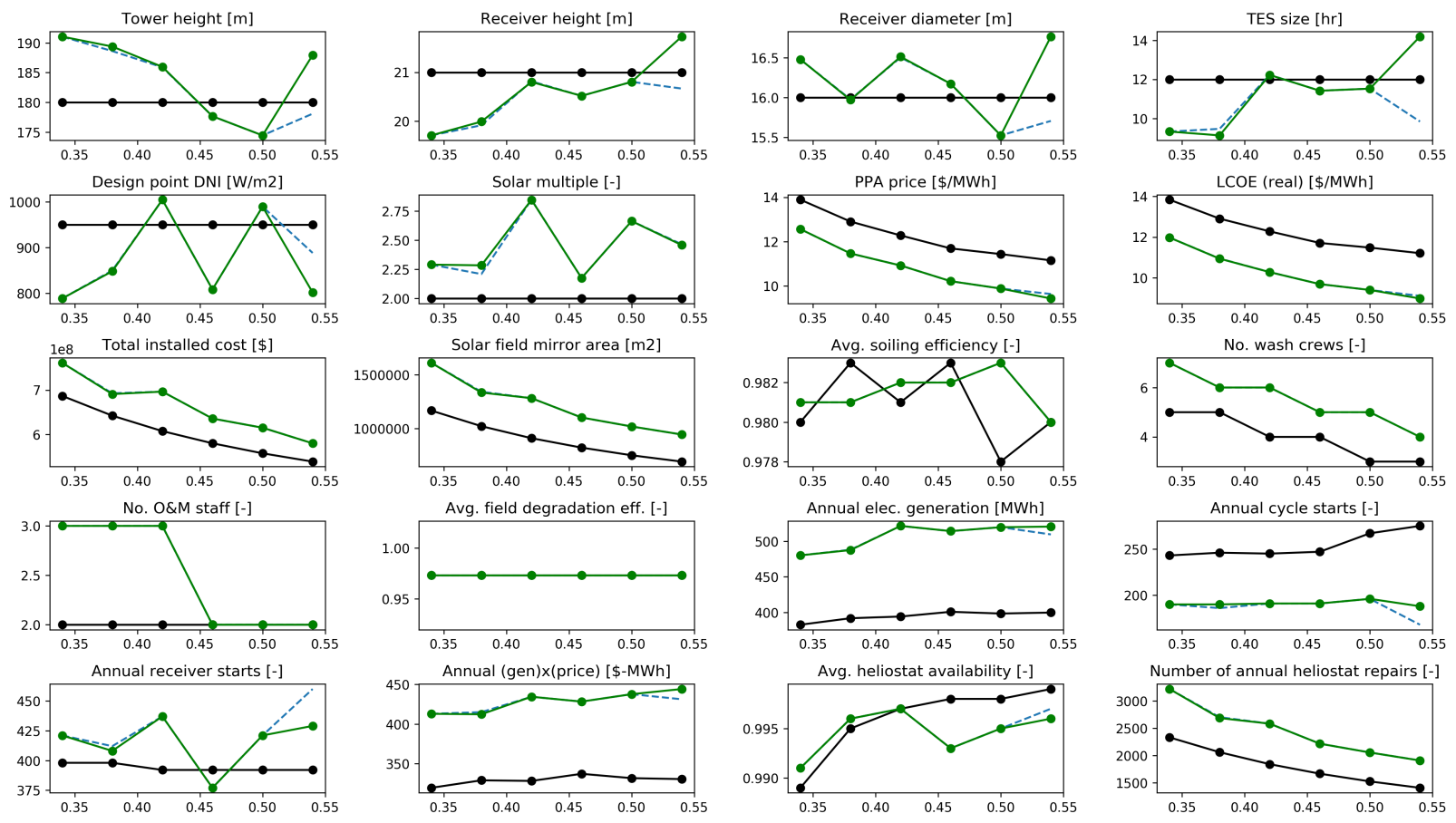

Figure 48. Results of system optimization over a range of nominal power cycle conversion efficiencies. The plot shows the starting-point value in black and the best value in green. The final value at convergence is shown in dotted blue (often covered by the green lines).

- System optimization substantially improves the PPA price and LCOE by $15-20 \%$, especially in cases where the design-point efficiency deviates most from the current state-of-the-art.

- The PPA price response surface offers a relatively broad region near the optimal point, so it is possible to use a variety of designs to achieve a PPA price that is close to optimal. This is reflected in the variability of the optimal variable values with corresponding low-variability trends for PPA price and LCOE.

- The number of cycle starts is reduced substantially reduced in each case by the optimizer, and this indicates that limiting power cycle starts is an important aspect of successful long-term plant operation.

- The PPA price improves as efficiency increases, but the rate of improvement decreases at higher efficiency. The muted impact of efficiency gain is likely due to the increasing relatively importance of fixed and O\&M costs as capital costs drop.

Summary results from the three remaining cases are presented in Figure 49, where selected variable and metric data are plotted. Figure 3.49(a) shows the impact of heliostat MTTF, with the primary result being that the rate of failure is largely accounted for by adding or removing O\&M staff dedicated to making repairs, while there is a minor incentive to overbuild the field at high rates of failure. Note that this conclusion may be significantly affected by heliostat cost assumptions, since the assumed baseline cost for this study was relatively high at $\$ 145 / \mathrm{m}^{2}$. Figure 3.49(b) shows the impact of heliostat specific cost, all other factors equal. The results show that increased cost leads to a smaller heliostat field, and this is largely achieved by reducing the solar multiple while counteracting some of the 
reduction by also decreasing the design-point DNI (decreased design-point DNI results in an increased heliostat field size relative to the thermal rating of the receiver). Finally, Figure 3.49(c) shows the impact of heliostat soiling rate. Most notable is the important role of number of wash crews in addressing the impact and the relatively low impact of soiling on PPA price once the optimization has been completed. High rates of soiling are also counteracted by increased solar field area.
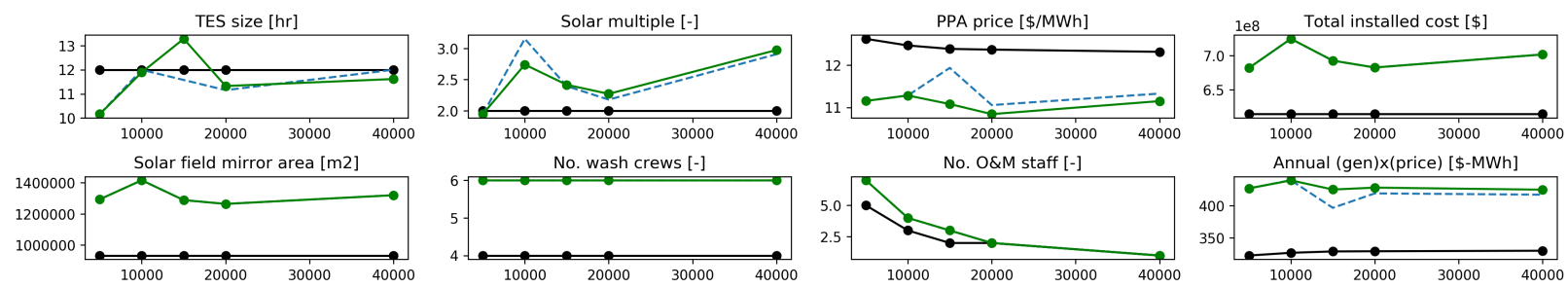

(a) Heliostat mean-time-to-failure (hr)
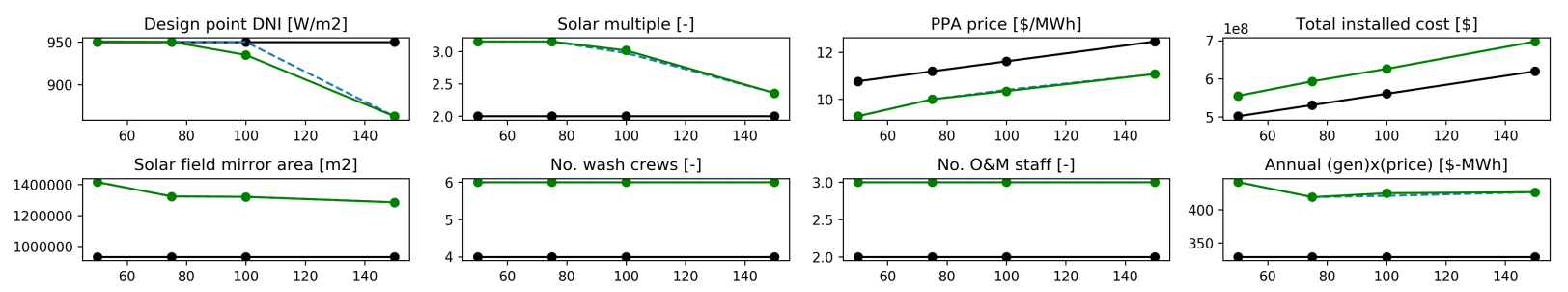

(b) Heliostat specific cost $\left(\$ / \mathrm{m}^{2}\right)$
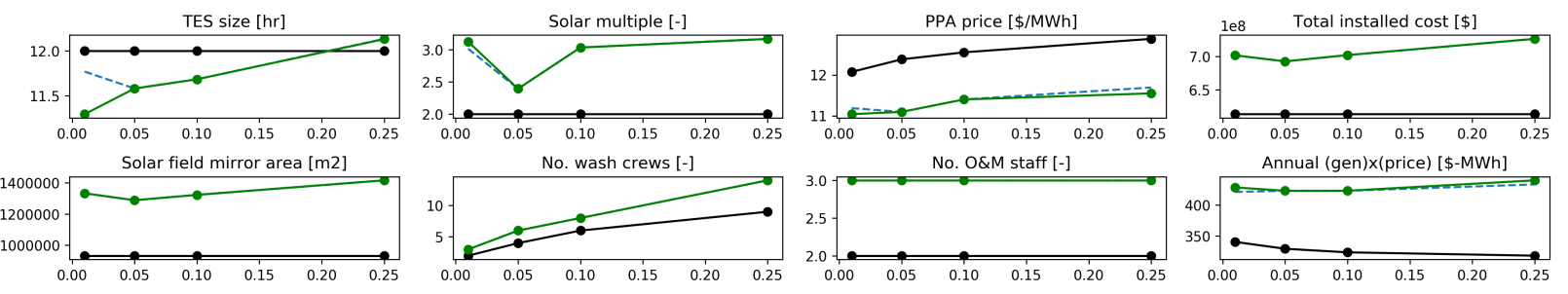

(c) Mirror soiling rate $(1 /$ week $)$

Figure 49. Summary results from three parametric studies.

While the results do show some clear trends, additional improvement to the optimization algorithms and operating constraints is necessary to improve the clarity of the output. For example, the maximum receiver operating limit is a function of design-point DNI, but the default assumption of 1.2 times the design-point mass flow rate may have allowed the solver to unrealistically decrease design-point DNI in some cases. Refinement to convergence criteria is also warranted due to scatter in the optimized variable values. Nonetheless, the fundamental conclusion that systematic and holistic optimization can identify improved designs has been reinforced with these analyses. 


\section{Summary, conclusions, and future work}

The work presented in this report outlines the outcomes that we have achieved in developing a system-level design and operations optimization package.

Specifically, we have:

1. Improved our dispatch optimization algorithm, applying it to cases of commercial interest with industry partner SolarReserve

2. Identified key operational parameters that affect PPA price and other metrics of interest

3. Developed a stochastic dispatch model that follows a two-stage approach

4. Developed a stochastic forecasting tool that is capable of producing realistic forecast scenarios for use in the dispatch model

5. Identified a novel technique for optimizing derivative-free nonlinear integer problems and begun investigating the optimization of CSP systems with PPA price as an objective

6. Developed a simulation-based power cycle availability and failure model that considers individual components' lifetime and failure probabilities

7. Developed a technique that significantly reduces computation time while preserving trends in the response of the objective function to changing variable values

8. Completed a mirror washing and replacement procedure optimization program that solves for the number of wash crews required while maximizing heliostat field reflectivity

9. Completed substantial development of a script-based user interface that allows users to conduct a wide range of simulation and optimization activities

10. Demonstrated the holistic optimization capability of the tools that we have developed.

Recommendations for future work include additional analysis of optimal design for different market and power rating conditions, continued collection of data that can be used to refine model parameters, active transition of the software into the public domain, and refinement of the script interface to provide more intuitive functionality and helpful examples. 


\section{Bibliography}

Abramson, M. A. "Mixed variable optimization of a load-bearing thermal insulation system using a filter pattern search algorithm". Optimization and Engineering 5 (2004): 157-177.

Aguilar, Oscar, et al. "Availability and reliability considerations in the design and optimisation of flexible utility systems”. Chemical Engineering Science 63, no. 14 (2008): 3569-3584.

Akhil, Abbas Ali, et al. DOE/EPRI Electricity Storage Handbook in Collaboration with NRECA. [inlangen]. Tech. rep. SAND2015-1002, 1170618. Feb. 2015. Visited on 04/11/2018. https : / / doi . org / 10 . 2172 / 1170618. http://www.osti.gov/servlets/purl/1170618/.

Almanza, R., et al. "Development and mean life of aluminum first-surface mirrors for solar energy applications". Solar Energy Materials and Solar Cells 93, no. 9 (2009): 1647-1651. https : / / doi . org/https: / / doi . org/10.1016/j.solmat.2009.05.004.

Alon, L., et al. "Computer-Based Management of Mirror-Washing in Utility-Scale Solar Thermal Plants". Proceedings of Energy Sustainability, Volume 1: Combined Energy Cycles, CHP, CCHP, and Smart Grids; Concentrating Solar Power, Solar Thermochemistry and Thermal Energy Storage; Geothermal, Ocean, and Emerging Energy Technologies; Hydrogen Energy Technologies; Low/Zero Emission Power Plants and Carbon Sequestration; Photovoltaics; Wind Energy Systems and Technologies ES2014-6562 (2014): 1-10.

Altés Buch, Queralt. Dynamic modeling of a steam Rankine Cycle for concentrated solar power applications. PhD thesis, Polytechnic University of Catalonia, Barcelona, Spain, 2014.

Aminifar, Farrokh, Mahmud Fotuhi-Firuzabad, and Mohammad Shahidehpour. "Unit commitment with probabilistic spinning reserve and interruptible load considerations”. IEEE Transactions on Power Systems 24, no. 1 (2009): 388-397.

Aminov, RZ, and AI Kozhevnikov. "Optimization of the operating conditions of gas-turbine power stations considering the effect of equipment deterioration”. Thermal Engineering 64, no. 10 (2017): 715-722.

Andradóttir, Sigrún. "An overview of simulation optimization via random search". Handbooks in operations research and management science 13 (2006): 617-631.

Andreas, A., and T. Stoffel. University of Nevada (UNLV): Las Vegas, Nevada (Data). Tech. rep. NREL Report No. DA-5500-56509. 2006. Visited on 12/06/2018. http: / / dx. doi .org/10.5439/1052548.

April, Jay, et al. "Practical introduction to simulation optimization". In Proceedings of the 35th conference on Winter simulation: driving innovation, 71-78. Winter Simulation Conference, 2003.

Arthur, D., and S. Vassilvitskii. “K-means++: The advantages of careful seeding”. In Proceedings of the Annual ACM-SIAM Symposium on Discrete Algorithms, 7:1027-1035. 2007.

Audet, C., and J. E. Dennis. "Pattern Search Algorithms for Mixed Variable Programming". SIAM J. on Optimization 11, no. 3 (2000): 573-594. ISSN: 1052-6234.

Barringer, P. The Weibull Failure Database. HTTP://WWW.BARRINGER1.COM/WDBASE.HTM, accessed February 29, 2016, 2010.

Benato, Alberto, Anna Stoppato, and Stefano Bracco. “Combined cycle power plants: A comparison between two different dynamic models to evaluate transient behaviour and residual life". Energy Conversion and Management 87 (2014): 1269-1280.

Bergeron, K.D., and J.M. Freese. "Cleaning strategies for parabolic-trough solar-collector fields; guidelines for decisions". NASA STI/Recon Technical Report N 81 (June 1981).

Berkelaar, Michel, Kjell Eikland, and Peter Notebaert. "LPSolve: Open Source (Mixed-Integer) Linear Programming System". Eindhoven U. of Technology (2004).

Bezdek, J. C., R. Ehrlich, and W. Full. "FCM: The fuzzy c-means clustering algorithm". Computers and Geosciences 10, numbers 2-3 (1984): 191-203.

Blair, Nate, et al. System Advisor Model, SAM 2014.1.14 : General Description. Tech. rep. February. Golden, CO: National Renewable Energy Laboratory, 2014.

Blanco, C., and D. Soronow. "Jump diffusion processes: energy price processes used for derivatives pricing and risk management”. Commodities Now September (2001): 83-78. 
- . "Mean reverting processes: energy price processes used for derivatives pricing and risk management". Commodities Now June (2001): 68-72.

Bonami, P., et al. "An algorithmic framework for convex mixed integer nonlinear programs". Discrete Optimization 5, no. 2 (2008): 186-204.

Borgonovo, Emanuele, Marzio Marseguerra, and Enrico Zio. "A Monte Carlo methodological approach to plant availability modeling with maintenance, aging and obsolescence". Reliability Engineering \& System Safety 67, no. 1 (2000): 61-73.

Chang, Daofang, et al. "Developing a dynamic rolling-horizon decision strategy for yard crane scheduling". Advanced Engineering Informatics 25, no. 3 (2011): 485-494.

Chen, Chun-Hung, Michael C Fu, and Leyuan Shi. "Simulation and optimization". In State-of-the-Art DecisionMaking Tools in the Information-Intensive Age, 247-260. Informs, 2008.

Cho, Heejin, et al. "Evaluation of CCHP systems performance based on operational cost, primary energy consumption, and carbon dioxide emission by utilizing an optimal operation scheme". Applied Energy 86, no. 12 (2009): 2540-2549.

Ciocan, Dragos Florin, and Vivek Farias. "Model predictive control for dynamic resource allocation". Mathematics of Operations Research 37, no. 3 (2012): 501-525.

Cohen, G.E., David W Kearney, and Gregory J. Kolb. Final Report on the Operation and Maintenance Improvement Program for Concentrating Solar Power Plants. Tech. rep. June. Albuquerque, NM, 1999.

Deffenbaugh, D.M., S.T. Green, and S.J. Svedeman. "The effect of dust accumulation on line-focus parabolic trough solar collector performance". Solar Energy 36, no. 2 (1986): 139-146. https : / / doi . org / https : //doi.org/10.1016/0038-092x(86) 90118-0.

Denholm, P. Enabling greater penetration of solar power via the use of CSP with thermal energy storage. Ed. by Mark S Mehos. NREL/TP ; 6A20-52978. Golden, CO, 2011.

Denholm, Paul, and Mark Mehos. Enabling Greater Penetration of Solar Power via the Use of CSP With Thermal Energy Storage. Tech. rep. November. Golden, CO, 2011.

Department of the Army. Survey of Reliability and Availability Information for Power Distribution, Power Generation, and Heating, Ventilating and Air Conditioning (HVAC) Components for Commercial, Industrial, and Utility Installations. Tech. rep. HQ, Department of the Army, 2006.

Dowling, Alexander W, Ranjeet Kumar, and Victor M Zavala. "A multi-scale optimization framework for electricity market participation". Applied Energy 190 (2017): 147-164.

Dunham, Marc T., and Brian D. Iverson. "High-Efficiency Thermodynamic Power Cycles for Concentrated Solar Power Systems". Renewable and Sustainable Energy Reviews 30 (2014): 758-770.

Duran, M. A., and I. Grossmann. "An Outer-Approximation Algorithm for a Class of Mixed-Integer Nonlinear Programs". Mathematical Programming 36 (1986): 307-339.

Eide, SA, et al. "Industry-average performance for components and initiating events at US commercial nuclear power plants". Idaho National Laboratory, US Nuclear Regulatory Commission Office of Nuclear Regulatory Research Washington, DC (2007): 20555-0001.

Exler, O., and K. Schittkowski. "A Trust Region SQP Algorithm for Mixed-Integer Nonlinear Programming”. Optimization Letters 1 (2007): 269-280.

Fernández-García, A., et al. "Study of Different Cleaning Methods for Solar Reflectors Used in CSP Plants". Proceedings of the SolarPACES 2013 International Conference, Energy Procedia 49 (2014): 80-89. https : // doi.org/https: // doi.org/10.1016/j.egypro.2014.03.009.http: // www . sciencedirect.com/science/article/pii/s1876610214004639.

Fishman, George S. Monte Carlo: Concepts, Algorithms, and Applications. Springer, 1996.

Forbes, Catherine, et al. Statistical distributions. John Wiley \& Sons, 2011.

Fourer, Robert, David Gay, and Brian Kernighan. Ampl. Vol. 119. Boyd \& Fraser, 1993.

Frangopoulos, Christos A., and George G. Dimopoulos. "Effect of reliability considerations on the optimal synthesis, design and operation of a cogeneration system". Energy 29 (3 2004): 309-329.

Frey, B. J., and D. Dueck. "Clustering by passing messages between data points". Science 315, no. 5814 (2007): 972-976. 
Fu, Michael C, Fred W Glover, and Jay April. "Simulation optimization: a review, new developments, and applications". In Proceedings of the Winter Simulation Conference, 2005. 13-pp. IEEE, 2005.

Fürsch, Michaela, et al. "The role of grid extensions in a cost-efficient transformation of the European electricity system until 2050". Applied Energy 104 (2013): 642-652.

García-Segura, A., et al. "Durability studies of solar reflectors: A review". Renewable and Sustainable Energy Reviews 62 (2016): 453-467. https://doi.org/https://doi.org/10.1016/j.rser.2016.04. 060. http://www.sciencedirect.com/science/article/pii/s1364032116300934.

Gee, R., et al. An improved method for characterizing reflector specularity for parabolic trough concentrators, 2010.

Geoffrion, A. M. "Generalized Benders Decomposition”. Journal of Optimization Theory and Applications 10, no. 4 (1972): 237-260.

Gilman, Paul, and Aron Dobos. System Advisor Model, General Description. Tech. rep. February. Golden, CO: National Renewable Energy Laboratory, 2012.

Godoy, Ezequiel, Sonia J Benz, and Nicolás J Scenna. "An optimization model for evaluating the economic impact of availability and maintenance notions during the synthesis and design of a power plant". Computers \& Chemical Engineering 75 (2015): 135-154.

Goldberg, D. E. Genetic algorithms in search, optimization, and machine learning. Boston: Addison-Wesley, 1989.

Green, A., et al. "High Capacity Factor CSP-PV Hybrid Systems". Energy Procedia, International Conference on Concentrating Solar Power and Chemical Energy Systems, SolarPACES 2014, 69 (May 2015): 2049-2059. ISSN: 1876-6102, visited on 10/06/2016. https : / / doi . org / 10 . 1016 / j . egypro. 2015 . 03 . 218. http://www.sciencedirect.com/science/article/pii/s187661021500524X.

Guédez, Rafael, James Spelling, and Björn Laumert. "Reducing the number of turbine starts in concentrating solar power plants through the integration of thermal energy storage". Journal of Solar Energy Engineering 137, no. 1 (2015): 011003.

Guédez, Rafael, et al. "Reducing the Number of Turbine Starts in Concentrating Solar Power Plants Through the Integration of Thermal Energy Storage". In Proceedings of the ASME Turbo Expo 2013, 1-10. San Antonio, Texas, 2013.

Guédez, Rafael, et al. "A Methodology for Determining Optimum Solar Tower Plant Configurations and Operating Strategies to Maximize Profits". In Proceedings of the ASME 2015 9th International Conference on Energy Sustainability, 1-15. San Diego, CA: American Society of Mechanical Engineers, 2015.

Haghifam, Mahmood Reza, and Moein Manbachi. "Reliability and availability modelling of combined heat and power (CHP) systems". International Journal of Electrical Power and Energy Systems 3, no. 33 (2011): 385-393.

Hale, PS, and RG Arno. "Survey of reliability and availability information for power distribution, power generation, and HVAC components for commercial, industrial, and utility installations". IEEE Transactions on Industry Applications 37, no. 1 (2001): 191-196.

Hedman, Kory W, et al. "Co-optimization of generation unit commitment and transmission switching with N-1 reliability”. IEEE Transactions on Power Systems 25, no. 2 (2010): 1052-1063.

Higle, Julia L, and Suvrajeet Sen. "On the convergence of algorithms with implications for stochastic and nondifferentiable optimization". Mathematics of Operations Research 17, no. 1 (1992): 112-131.

Hirsch, Tobias, et al. "Standardization of CSP Performance Model Projection - Latest Results From the Stamp Project". In Proceedings of the ASME 2011 International Conference on Energy Sustainability, 1-6. 2011.

Hunter, S.R., et al. "Low cost anti-soiling coatings for CSP collector mirrors and heliostats". Proceedings of the Society of Photo-Optical Instrumentation Engineers, no. 9175 (2014): 1-12.

IBM. IBM ILOG CPLEX V12.1 User's Manual for CPLEX. Armonk, NY, 2009.

IEEE. "IEEE recommended practice for the design of reliable industrial and commercial power systems". 2007.

Ineichen, Pierre, and Richard Perez. "A new airmass independent formulation for the Linke turbidity coefficient". Solar Energy 73, no. 3 (2002): 151-157.

Joglekar, N., et al. "A Levelized Cost Metric for EDS-Based Cleaning of Mirrors in CSP Power Plants". Proceedings of Energy Sustainability, Volume 1: Combined Energy Cycles, CHP, CCHP, and Smart Grids; Concentrating Solar Power, Solar Thermochemistry and Thermal Energy Storage; Geothermal, Ocean, and Emerging Energy Technologies; Hydrogen Energy Technologies; Low/Zero Emission Power Plants and Carbon Sequestration; Photovoltaics; Wind Energy Systems and Technologies ES2014-6496 (2014): 1-7.

111

This report is available at no cost from the National Renewable Energy Laboratory (NREL) at www.nrel.gov/publications 
Jorgenson, J, P Denholm, and M Mehos. Estimating the Value of Utility-Scale Solar Technologies in California Under a 40 \% Renewable Portfolio Standard. Tech. rep. May. Golden, CO: National Renewable Energy Laboratory, 2014.

Jung, June Young, et al. "A simulation based optimization approach to supply chain management under demand uncertainty”. Computers \& Chemical Engineering 28, no. 10 (2004): 2087-2106.

Kaur, Amanpreet, Hugo TC Pedro, and Carlos FM Coimbra. "Impact of onsite solar generation on system load demand forecast". Energy conversion and management 75 (2013): 701-709.

Keatley, Patrick. "Cost modelling of coal power plant start-up in cyclical operation". In Coal Power Plant Materials and Life Assessment, 358-388. 2014.

Keatley, Patrick, Ahmed Shibli, and NJ Hewitt. "Estimating power plant start costs in cyclic operation". Applied Energy 111 (2013): 550-557.

Kim, Seong-Hee, and Barry L Nelson. "Selecting the best system". Handbooks in operations research and management science 13 (2006): 501-534.

Kleijnen, Jack PC. "Response surface methodology for constrained simulation optimization: An overview". Simulation Modelling Practice and Theory 16, no. 1 (2008): 50-64.

Kolb, G.J., et al. Heliostat Cost Reduction Study. Tech. rep. SAND2007-3293. Albuquerque, New Mexico: Sandia National Laboratories, 2007.

Kost, Christoph, Christoph M Flath, and Dominik Möst. "Concentrating solar power plant investment and operation decisions under different price and support mechanisms". Energy Policy 61 (2013): 238-248.

Kumar, N, et al. "Power Plant Cycling Costs". Technical Report, National Renewable Energy Lab 303 (2012): 2753000.

Kumar, Ravinder, A Sharma, and P Tewari. "Markov approach to evaluate the availability simulation model for power generation system in a thermal power plant". International Journal of Industrial Engineering Computations 3, no. 5 (2012): 743-750.

Kushner, Harold, and G George Yin. Stochastic approximation and recursive algorithms and applications. Vol. 35. Springer Science \& Business Media, 2003.

Lata, Jesús M., Manuel Rodríguez, and Mónica Álvarez de Lara. "High Flux Central Receivers of Molten Salts for the New Generation of Commercial Stand-Alone Solar Power Plants". Journal of Solar Energy Engineering 130, no. 2 (2008): 021002.

Law, Edward W, Merlinde Kay, and Robert A Taylor. "Calculating the financial value of a concentrated solar thermal plant operated using direct normal irradiance forecasts". Solar Energy 125 (2016): 267-281.

Law, Edward W, et al. "Direct normal irradiance forecasting and its application to concentrated solar thermal output forecasting-A review". Solar Energy 108 (2014): 287-307.

Le, KD, and JT Day. "Rolling horizon method: A new optimization technique for generation expansion studies". IEEE Transactions on Power Apparatus and Systems, no. 9 (1982): 3112-3116.

Liu, Yangyang, et al. "Optimal dispatch of virtual power plant using interval and deterministic combined optimization”. International Journal of Electrical Power \& Energy Systems 102 (2018): 235-244.

Lizarraga-Garcia, Enrique, et al. "Optimal operation of a solar-thermal power plant with energy storage and electricity buy-back from grid". Energy 51 (2013): 61-70.

Madaeni, Seyed Hossein, et al. "How Thermal Energy Storage Enhances the Economic Viability of Concentrating Solar Power". Proceedings of the IEEE 10, no. 2 (2012): 335-347.

Mahajan, Ashutosh, et al. MINOTAUR: A toolkit for solving mixed-integer nonlinear optimization. wiki-page. Http://wiki.mcs.anl.gov/minotaur, 2011.

Mak, Wai-Kei, David P Morton, and R Kevin Wood. "Monte Carlo bounding techniques for determining solution quality in stochastic programs". Operations research letters 24, numbers 1-2 (1999): 47-56.

Mani, M., and R. Pillai. "Impact of dust on solar photovoltaic (PV) performance: Research status, challenges and recommendations". Renewable and Sustainable Energy Reviews 14, no. 9 (2010): 3124-3131. https : / / doi . org/https://doi.org/10.1016/j.rser.2010.07.065.http://www.sciencedirect.com/ science/article/pii/S1364032110002455.

Margolis, R, C Coggeshall, and J Zuboy. "Sunshot Vision Study”. US Dept. of Energy, no. February (2012).

112

This report is available at no cost from the National Renewable Energy Laboratory (NREL) at www.nrel.gov/publications 
Marquez, R., and C. F. M. Coimbra. "Forecasting of global and direct solar irradiance using stochastic learning methods, ground experiments and the NWS database". Solar Energy 85 (2011): 746-756.

Marseguerra, Marzio, and Enrico Zio. "Optimizing maintenance and repair policies via a combination of genetic algorithms and Monte Carlo simulation". Reliability Engineering \& System Safety 68, no. 1 (2000): 69-83.

Martinez-Rojas, Marcela, et al. "Reactive power dispatch in wind farms using particle swarm optimization technique and feasible solutions search". Applied Energy 88, no. 12 (2011): 4678-4686.

Mazumder, M., et al. "Development of Self-Cleaning Solar Collectors for Minimizing Energy Yield Loss Caused by Dust Deposition". ASME 2013 7th International Conference on Energy Sustainability ES2013-18365 (2013): $1-10$.

Mazumder, M.K., et al. "Self-Cleaning Solar Mirrors Using Electrodynamic Dust Shield: Prospects and Progress". Proceedings of Energy Sustainability, Volume 1: Combined Energy Cycles, CHP, CCHP, and Smart Grids; Concentrating Solar Power, Solar Thermochemistry and Thermal Energy Storage; Geothermal, Ocean, and Emerging Energy Technologies; Hydrogen Energy Technologies; Low/Zero Emission Power Plants and Carbon Sequestration; Photovoltaics; Wind Energy Systems and Technologies ES2014-6696 (2014): 1-10.

Mehos, M., et al. Parameters and method to evaluate the solar reflectance properties of reflector materials for concentrating solar power technology. Tech. rep. NREL/TP-5500-67464. National Renewable Energy Laboratory, 2017.

Mellit, A., et al. "An adaptive model for predicting of global, direct and diffuse hourly solar irradiance". Energy Conversion and Management 51, no. 4 (2010): 771 -782. ISSN: 0196-8904. https : / / doi . org / http : //dx.doi.org/10.1016/j.enconman.2009.10.034.http://www.sciencedirect.com/ science/article/pii/s0196890409004427.

Menéndez, Rogelio Peón, et al. "A Novel Modeling of Molten-Salt Heat Storage Systems in Thermal Solar Power Plants". Energies 7 (10 2014): 6721-6740.

Meyen, S., et al. Parameters and method to evaluate the solar reflectance properties of reflector materials for concentrating solar power technology. Tech. rep. SolarPACES Official Reflectance Guideline Version 2.5, 2013.

Mirandola, A., A. Stoppato, and E. Lo Casto. "Evaluation of the effects of the operation strategy of a steam power plant on the residual life of its devices". Energy 35 (2 2010): 1024-1032.

National Renewable Energy Laboratory. System Advisor Model, 2012.

Neises, T, and C Turchi. "A Comparison of Supercritical Carbon Dioxide Power Cycle Configurations With an Emphasis on CSP Applications”. Energy Procedia 49 (2014): 1187-1196.

Nonnenmacher, Lukas, Amanpreet Kaur, and Carlos FM Coimbra. "Day-ahead resource forecasting for concentrated solar power integration". Renewable Energy 86 (2016): 866-876.

Nosrat, Amir, and Joshua M Pearce. "Dispatch strategy and model for hybrid photovoltaic and trigeneration power systems". Applied Energy 88, no. 9 (2011): 3270-3276.

Nostell, P., A. Roos, and B. Karlsson. "Ageing of solar booster reflector materials". Solar Energy Materials and Solar Cells 54, no. 1 (1998): 235-246.

Olafsson, Sigurdur, Xiaonan Li, and Shuning Wu. "Operations research and data mining". European Journal of Operational Research 187, no. 3 (2008): 1429-1448.

O'Neill, Richard P, et al. "Economic analysis of the N-1 reliable unit commitment and transmission switching problem using duality concepts". Energy Systems 1, no. 2 (2010): 165-195.

Pan, Christoph Adrian, and Frank Dinter. "Combination of PV and central receiver CSP plants for base load power generation in South Africa". Solar Energy 146, no. Supplement C (Apr. 2017): 379-388. ISSN: 0038-092X. https://doi.org/10.1016/j.solener.2017.02.052.http://www.sciencedirect.com/ science/article/pii/s0038092X17301536.

Pandžić, Hrvoje, Igor Kuzle, and Tomislav Capuder. "Virtual power plant mid-term dispatch optimization". Applied Energy 101 (2013): 134-141.

Panneton, François, Pierre L'Ecuyer, and Makoto Matsumoto. "Improved long-period generators based on linear recurrences modulo 2". ACM Transactions on Mathematical Software (TOMS) 32, no. 1 (2006): 1-16.

Parisio, Alessandra, et al. "Use of model predictive control for experimental microgrid optimization". Applied Energy 115 (2014): 37-46. 
Perera, ATD, et al. "A hybrid tool to combine multi-objective optimization and multi-criterion decision making in designing standalone hybrid energy systems". Applied Energy 107 (2013): 412-425.

Perez, Richard, et al. "A new operational model for satellite-derived irradiances: description and validation". Solar Energy 73, no. 5 (2002): 307-317.

Pettit, R. B. "Characterization of the reflected beam profile of solar mirror materials". Solar Energy 19, no. 6 (1977): 733-741.

Powell, Kody M, and Thomas F Edgar. "Modeling and control of a solar thermal power plant with thermal energy storage". Chemical Engineering Science 71 (2012): 138-145.

Pruitt, Kristopher A., Robert J. Braun, and Alexandra M. Newman. "Evaluating Shortfalls in Mixed-Integer Programming Approaches for the Optimal Design and Dispatch of Distributed Generation Systems". Applied Energy 102 (2013): 386-398.

Reikard, Gordon. "Predicting solar radiation at high resolutions: A comparison of time series forecasts". Solar Energy 83, no. 3 (2009): 342-349.

Röger, M., et al. "Techno-economic analysis of receiver replacement scenarios in a parabolic trough field". AIP Conference Proceedings 1734, no. 1 (2016): 030030.

Sabouhi, Hamed, et al. "Reliability modeling and availability analysis of combined cycle power plants". International Journal of Electrical Power \& Energy Systems 79 (2016): 108-119.

Santos, Maria Izabel, and Wadaed Uturbey. "A practical model for energy dispatch in cogeneration plants". Energy 151 (2018): 144-159.

Sarver, T., A. Al-Qaraghuli, and L.L. Kazmerski. "A comprehensive review of the impact of dust on the use of solar energy: History, investigations, results, literature, and mitigation approaches". Renewable and Sustainable Energy Reviews 22 (2013): 698-733. https: //doi.org/https://doi.org/10.1016/j.rser.2012.12. 065.

Shah, Devavrat, and Damon Wischik. "Switched networks with maximum weight policies: Fluid approximation and multiplicative state space collapse". The Annals of Applied Probability 22, no. 1 (2012): 70-127.

Silvente, Javier, et al. "A rolling horizon optimization framework for the simultaneous energy supply and demand planning in microgrids". Applied Energy 155 (2015): 485-501.

Singh, Dileep, et al. "Analysis of a Graphite Foam-Nacl Latent Heat Storage System for Supercritical $\mathrm{CO}_{2}$ Power Cycles for Concentrated Solar Power". Solar Energy 118 (2015): 232-242.

Sioshansi, Ramteen. "The Value of Concentrating Solar Power and Thermal Energy Storage". IEEE Transactions on Energy 1, no. 3 (2010): 173-183.

Stoppato, A., et al. "On the operation strategy of steam power plants working at variable load: Technical and economic issues". Energy 37 (1 2012): 228-236.

Thorin, Eva, Heike Brand, and Christoph Weber. "Long-term optimization of cogeneration systems in a competitive market environment". Applied Energy 81, no. 2 (2005): 152-169.

Tică, Adrian, et al. "Design of a combined cycle power plant model for optimization". Applied Energy 98 (2012): 256-265.

Truong Ba, H., et al. "Optimal condition-based cleaning of solar power collectors”. Solar Energy 157 (2017): 762 777.

Tsay, R. S. Analysis of financial time series. Vol. 543. John Wiley \& Sons, 2005.

Turchi, Craig, et al. "Current and Future Costs for Parabolic Trough and Power Tower Systems in the US Market Preprint". Renewable Energy, no. October (2010): 11.

Turchi, Craig S, Zhiwen Ma, and John Dyreby. "Supercritical carbon dioxide power cycle configurations for use in concentrating solar power systems". In ASME Turbo Expo 2012: Turbine Technical Conference and Exposition, 967-973. American Society of Mechanical Engineers, 2012.

Uniejewski, Bartosz, Rafal Weron, Florian Ziel, et al. Variance stabilizing transformations for electricity spot price forecasting. Tech. rep. Hugo Steinhaus Center, Wroclaw University of Technology, 2017.

Usaola, J. "Operation of concentrating solar power plants with storage in spot electricity markets". IET renewable power generation 6, no. 1 (2012): 59-66.

Wagner, Michael J. Simulation and Predictive Performance Modeling of Utility-Scale Central Receiver System Power Plants. Masters Thesis, University of Wisconsin - Madison, 2008. 
Wagner, Michael J., and Paul Gilman. Technical Manual for the SAM Physical Trough Model. Tech. rep. June. Golden, CO: National Renewable Energy Laboratory, 2011.

Wagner, Michael J, et al. "Dispatching Power at a Concentrated Solar Energy Facility". Working Paper (2017).

Wagner, Michael J, et al. "Optimized dispatch in a first-principles concentrating solar power production model". Applied Energy 203 (2017): 959-971.

Wagner, M.J., and T. Wendelin. "SolarPILOT: A power tower solar field layout and characterization tool". Solar Energy 171 (2018): 185-196. https://doi.org/https://doi.org/10.1016/j.solener.2018. 06.063.

Westerlund, T., et al. "An extended cutting plane method for a class of non-convex MINLP problems". Computers \& Chemical Engineering 22 (1998): 357-365.

Whaley, Cass. Best Practices in Photovoltaic System Operations and Maintenance: 2 nd Edition [inlangen]. Tech. rep. NREL/TP-7A40-67553, 1336898. Dec. 2016. Visited on 04/11/2018. https : / / doi . org/ 10.2172 / 1336898. http://www.osti.gov/servlets/purl/1336898/.

Wild, Stefan M. Solving Derivative-Free Nonlinear Least Squares with POUNDERS. Preprint ANL/MCS-P51200414. Argonne Mathematics and Computer Science Division, 2014.

Wild, Stefan M., Rommel G. Regis, and Christine A. Shoemaker. "ORBIT: Optimization by Radial Basis Function Interpolation in Trust-Regions". SIAM J. Sci. Comput. 30, no. 6 (2008): 3197-3219.

Wolfertstetter, F., et al. "Integration of Soiling-Rate Measurements and Cleaning Strategies in Yield Analysis of Parabolic Trough Plants". Journal of Solar Energy Engineering 140, no. 4 (2018): 041008.

Wu, Hongyu, Mohammad Shahidehpour, and Mohammad E Khodayar. "Hourly demand response in day-ahead scheduling considering generating unit ramping cost". IEEE Transactions on Power Systems 28, no. 3 (2013): $2446-2454$.

Yuan, X.-X., M.D. Pandey, and G.A. Bickel. "A probabilistic model of wall thinning in CANDU feeders due to flow-accelerated corrosion”. Nuclear Engineering and Design 238, no. 1 (2008): 16-24. https : / doi . org/ https://doi.org/10.1016/j.nucengdes.2007.06.004.

Zare, V. "Exergoeconomic analysis with reliability and availability considerations of a nuclear energy-based combined cycle power plant". Energy 96 (2016): 187-196.

Zheng, Yu-Jun, et al. "Bio-inspired optimization of sustainable energy systems: A review". Mathematical Problems in Engineering 2013 (2013).

Zhu, G., D. Kearney, and M. Mehos. "On characterization and measurement of average solar field mirror reflectance in utility-scale concentrating solar power plants". Solar Energy 99 (2014): 185-202. 